Acta Crystallographica Section B

Structural

Science

ISSN 0108-7681

Editor: Carolyn P. Brock

\title{
Crystal and Molecular Structure of Thiocarbonyl-
} ethoxo(tetraphenylporphyrinato)ruthenium(II), $\left[\mathrm{Ru}(\mathrm{TPP})(\mathrm{CS})\left(\mathrm{HOC}_{2} \mathrm{H}_{5}\right)\right]$. A Case of Centrosymmetric-Noncentrosymmetric Ambiguity

T. J. Bartczak, K. Rachlewicz and L. Latos-Grazynski 


\title{
Crystal and Molecular Structure
}

of Thiocarbonyl-ethoxo(tetraphenylporphyrinato)

Ruthenium (II), [Ru(TPP)(CS)(HOC $\left.\left.\mathrm{H}_{5}\right)\right]$

A Case of Centrosymmetric - Noncentrosymmetric Ambiguity

\author{
BY TADEUSZ J. BARTCZAK* \\ X-Ray Crystallography Group
}

Institute of General and Ecological Chemistry, I-17

Technical University of Łódź - Politechnika Eódzka

ul. Żeromskiego 116, 90-924 Eódź, Poland

e-mail: tjbartcz@lodz1.p.lodz.pl

AND KRYSTYNA RACHLEWICZ

AND LECHOSŁAW LATOS-GRAŻYNSSKI

Department of Chemistry, University of Wroctaw,

ul. Joliot-Curie 14, 50-383 Wroctow, Poland

e-mail:1lg@ichuwr.chem.uni.wroc.pl
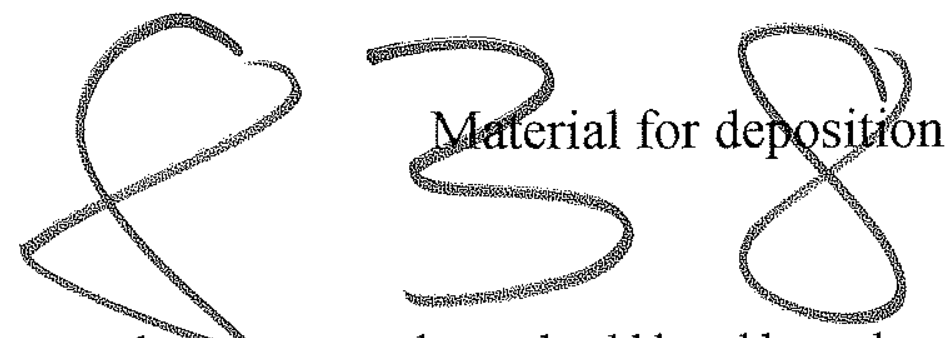

Authors to whom Eorrespondence should be addressed:

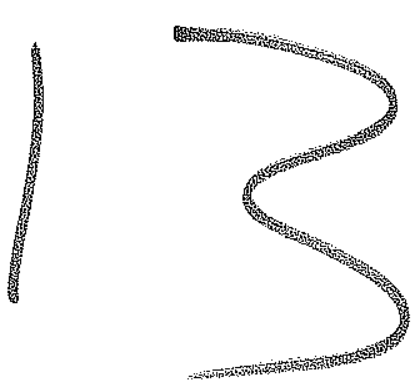

${ }^{*} \mathrm{X}$-ray crystallography (TJB), ${ }^{\#}$ synthetic and spetroscopic aspects (LL-G). 


\begin{abstract}
The compound $[\mathrm{Ru}(\mathrm{TPP})(\mathrm{CS})(\mathrm{EtOH})]$ crystallizes in the triclinic system.

Crystal data: $\mathrm{C}_{47} \mathrm{H}_{34} \mathrm{~N}_{4} \mathrm{ORuS}, M_{r}=803.91, a=10.606(3), b=11.308(5)$, $c=17.699(2) \mathrm{A}, \alpha=77.53(2), \beta=73.17(1), \gamma=69.85(3)^{\circ}, V=1891.6(10) \mathrm{A}^{3}, P-1$ $\left(C_{\mathrm{i}}^{1}\right.$, No. 2$), Z=2, F(000)=824, D_{x}=1.411, D_{m}=1.41$ (by flotation in aq. $\mathrm{KI}$ ) $\mathrm{Mg} \mathrm{m}^{-}$ ${ }^{3}, \mu(\mathrm{MoK \alpha})=0.512 \mathrm{~mm}^{-1}, \quad R=0.094, w R=0.097, s=2.30$ for 4611 independent reflections with $F_{o}>5 \sigma\left(F_{o}\right)$. Another data set was collected using $\mathrm{Cu} K \alpha$ radiation. The structure has been solved by direct and Patterson methods and refined by standard least-squares and difference Fourier methods in four models (centro- and noncentrosymmetric for $\mathrm{MoK} \alpha$ and $\mathrm{CuK \alpha}$ data sets). The best results were obtained for centrosymmetric description (space group $P-1$ ) and Mo data set. $\mathrm{Cu}$ data gave similar but less reliable results. The two independent $\mathrm{Ru}$ atoms lie in the special positions of the space group $P-1: 1 / 2,0,0$ and $1 / 2,1 / 2,1 / 2$. Consequently, the two independent molecules have crystallographically imposed -1 symmetry, the CS and EtOH axial groups are disordered at the opposite sides of the porphyrin macrorings with the site occupation factors constrained by the symmetry to 0.5 and the $\mathrm{RuN}_{4}$ portions of the molecules planar. The overall deviations from planarity of the porphinato core are very small. The $\mathrm{Ru}-\mathrm{C}-\mathrm{S}$ groups are essentially linear with the av. bond angle $\mathrm{Ru}-\mathrm{C}-\mathrm{S}=174(1)^{\circ}$. The av. dimensions of axial $\mathrm{CS}$ and EtOH groups are: $\mathrm{Ru}-\mathrm{C}(\mathrm{CS})=1.92(4)$ and $\mathrm{Ru}-\mathrm{O}(\mathrm{Et})=2.13(3) \mathrm{A}$
\end{abstract}


Table 3D. Atomic coordinates $\left(\times 10^{4}\right)$ and equivalent isotropic displacement coefficients $\left(\mathrm{A}^{2} \times 10^{3}\right)$

\begin{tabular}{|c|c|c|c|c|}
\hline Atom & $x$ & y & $z$ & $U_{e q}$ \\
\hline$S(1)$ & $8313(7)$ & $-87(10)$ & $-52(5)$ & $92(4)$ \\
\hline$C(45)$ & $6809(25)$ & $34(22)$ & $-44(12)$ & $34(6)$ \\
\hline $\operatorname{Ru}(1)$ & 5000 & 0 & 0 & $55(1)$ \\
\hline$O(1)$ & $3035(23)$ & $-295(19)$ & $112(12)$ & $71(7)$ \\
\hline$C(46)$ & $1996(49)$ & $506(46)$ & $-8(26)$ & $155(22)$ \\
\hline$C(47)$ & $1692(25)$ & $944(23)$ & $-834(14)$ & $92(7)$ \\
\hline$N(21)$ & $4134(7)$ & $1420(5)$ & $707(4)$ & $42(3)$ \\
\hline$N(22)$ & $5215(7)$ & $-1309(6)$ & $991(4)$ & $44(3)$ \\
\hline$C(1)$ & $3625(9)$ & $2717(7)$ & $456(5)$ & $47(4)$ \\
\hline$C(2)$ & $3070(9)$ & $3380(8)$ & $1132(6)$ & $56(4)$ \\
\hline$C(3)$ & $3233(9)$ & $2519(8)$ & $1790(6)$ & $57(4)$ \\
\hline$C(4)$ & $3938(9)$ & $1291(8)$ & $1503(5)$ & $49(4)$ \\
\hline$C(5)$ & $4261(9)$ & $137(8)$ & $2019(5)$ & $47(4)$ \\
\hline$C(22)$ & $4641(7)$ & $855(6)$ & $3140(4)$ & $72(5)$ \\
\hline$C(23)$ & 4344 & 982 & 3945 & $93(7)$ \\
\hline$C(24)$ & 3376 & 458 & 4495 & $94(7)$ \\
\hline$C(25)$ & 2704 & -194 & 4240 & $81(6)$ \\
\hline$c(26)$ & 3000 & -321 & 3435 & $66(5)$ \\
\hline$C(21)$ & 3969 & 203 & 2885 & $50(4)$ \\
\hline$C(6)$ & $4842(9)$ & $-1068(8)$ & $1762(5)$ & $48(4)$ \\
\hline$C(7)$ & $5277(10)$ & $-2237(8)$ & $2275(6)$ & $62(5)$ \\
\hline$C(8)$ & $5824(11)$ & $-3168(9)$ & $1803(6)$ & $65(5)$ \\
\hline$C(9)$ & $5795(8)$ & $-2605(7)$ & $988(5)$ & $45(4)$ \\
\hline$C(10)$ & $6333(9)$ & $-3226(7)$ & $340(5)$ & $48(4)$ \\
\hline$C(28)$ & $6160(5)$ & $-5483(5)$ & $637(4)$ & $63(5)$ \\
\hline$C(29)$ & 6772 & -6795 & 750 & $72(5)$ \\
\hline$C(30)$ & 8188 & -7292 & 702 & $97(7)$ \\
\hline$C(31)$ & 8993 & -6477 & 541 & $161(11)$ \\
\hline
\end{tabular}




\begin{tabular}{|c|c|c|c|c|}
\hline$C(32)$ & 8381 & -5165 & 428 & $145(10)$ \\
\hline$C(27)$ & 6964 & -4668 & 476 & $52(4)$ \\
\hline$S(2)$ & $6457(9)$ & $7452(7)$ & $4131(5)$ & $82(4)$ \\
\hline$C(145)$ & $5728(52)$ & $6409(36)$ & $4505(31)$ & $40(11)$ \\
\hline $\operatorname{Ru}(2)$ & 5000 & 5000 & 5000 & $68(1)$ \\
\hline$O(2)$ & $4438(45)$ & $3364(37)$ & $5661(27)$ & $95(16)$ \\
\hline$C(146)$ & $3201(61)$ & $3261(41)$ & $5609(31)$ & $266(43)$ \\
\hline$C(147)$ & $2833(39)$ & $2244(34)$ & $6157(22)$ & $148(16)$ \\
\hline$N(121)$ & $3029(7)$ & $6114(7)$ & $4985(5)$ & $56(3)$ \\
\hline$N(122)$ & $5172(8)$ & $4457(7)$ & $3941(4)$ & $57(4)$ \\
\hline$C(101)$ & $2108(10)$ & $6886(8)$ & $5558(5)$ & $59(4)$ \\
\hline$C(102)$ & $822(10)$ & $7509(9)$ & $5337(6)$ & $63(5)$ \\
\hline$C(103)$ & $934(11)$ & $7114(10)$ & $4643(6)$ & $69(5)$ \\
\hline$C(104)$ & $2330(10)$ & $6258(8)$ & $4413(6)$ & $55(4)$ \\
\hline$C(105)$ & $2819(10)$ & $5651(8)$ & $3732(5)$ & $57(4)$ \\
\hline$C(122)$ & $2100(7)$ & $6564(7)$ & $2451(5)$ & $77(6)$ \\
\hline$C(123)$ & 1251 & 6710 & 1944 & $96(7)$ \\
\hline$C(124)$ & 180 & 6163 & 2191 & $117(10)$ \\
\hline$C(125)$ & -43 & 5470 & 2945 & $129(11)$ \\
\hline$C(126)$ & 805 & 5324 & 3453 & $103(8)$ \\
\hline$C(121)$ & 1877 & 5871 & 3206 & $69(5)$ \\
\hline$C(106)$ & $4180(11)$ & $4815(8)$ & $3518(5)$ & $57(5)$ \\
\hline$C(107)$ & $4699(11)$ & $4171(8)$ & $2828(6)$ & $58(5)$ \\
\hline$C(108)$ & $5994(10)$ & $3443(8)$ & $2829(5)$ & $54(4)$ \\
\hline$C(109)$ & $6331(11)$ & $3589(9)$ & $3515(6)$ & $58(5)$ \\
\hline$C(110)$ & $7544(10)$ & $2950(8)$ & $3775(5)$ & $56(4)$ \\
\hline$C(128)$ & $8470(9)$ & $761(6)$ & $3411(4)$ & $123(8)$ \\
\hline$C(129)$ & 9373 & -147 & 2922 & $137(9)$ \\
\hline$C(130)$ & 10374 & 178 & 2279 & $88(6)$ \\
\hline$C(131)$ & 10472 & 1410 & 2124 & $102(7)$ \\
\hline$C(132)$ & 9570 & 2317 & 2613 & $91(7)$ \\
\hline$C(127)$ & 8569 & 1993 & 3257 & $60(4)$ \\
\hline
\end{tabular}


Equivalent isotropic $U$ defined as one third of the trace of the orthogonalized $U_{i j}$ tensor

Table 4D. Bond lengths (A)

$\begin{array}{lll}S(1)-C(45) & 1.549 & (29) \\ C(45)-R u(1) & 1.912 & (28) \\ R u(1)-O(1) & 2.169 & (26) \\ R u(1)-N(21) & 2.043 & (7) \\ R u(1)-N(22) & 2.049 & (6) \\ O(1)-C(46) & 1.201 & (49) \\ C(46)-C(47) & 1.529 & (54) \\ N(21)-C(1) & 1.397 & (9) \\ N(21)-C(4) & 1.346 & (12) \\ N(22)-C(6) & 1.365 & (11) \\ N(22)-C(9) & 1.380 & (10) \\ C(1)-C(2) & 1.420 & (14) \\ C(2)-C(3) & 1.359 & (13) \\ C(3)-C(4) & 1.455 & (12) \\ C(4)-C(5) & 1.419 & (11) \\ C(5)-C(21) & 1.487 & (11) \\ C(5)-C(6) & 1.403 & (12) \\ C(6)-C(7) & 1.445 & (11) \\ C(7)-C(8) & 1.350 & (14) \\ C(8)-C(9) & 1.450 & (13) \\ C(9)-C(10) & 1.352 & (13) \\ C(10)-C(27) & 1.529 & (9) \\ S(2)-C(145) & 1.557 & (51) \\ C(145)-R u(2) & 1.937 & (47) \\ R u(2)-O(2) & 2.137 & (43) \\ R u(2)-N(121) & 2.040 & (7) \\ R u(2)-N(122) & 2.036 & (8) \\ O(2)-C(146) & 1.387 & (88) \\ & & \end{array}$




$$
\begin{array}{lll}
C(146)-C(147) & 1.421 & (60) \\
N(121)-C(101) & 1.401 & (11) \\
N(121)-C(104) & 1.373 & (15) \\
N(122)-C(106) & 1.365 & (15) \\
N(122)-C(109) & 1.413 & (11) \\
C(101)-C(102) & 1.427 & (15) \\
C(102)-C(103) & 1.357 & (17) \\
C(103)-C(104) & 1.460 & (12) \\
C(104)-C(105) & 1.393 & (14) \\
C(105)-C(121) & 1.483 & (15) \\
C(105)-C(106) & 1.422 & (12) \\
C(106)-C(107) & 1.429 & (14) \\
C(107)-C(108) & 1.336 & (14) \\
C(108)-C(109) & 1.417 & (17) \\
C(109)-C(110) & 1.396 & (15) \\
C(110)-C(127) & 1.494 & (11) \\
C(110)-C(10 B) & 1.400 & (16) \\
T & \text { (1) }
\end{array}
$$




$$
\begin{aligned}
& \mathrm{C}(6)-\mathrm{N}(22)-\mathrm{C}(9) \quad 107.9(6) \\
& N(21)-C(1)-C(2) \quad 109.0(7) \\
& \mathrm{C}(1)-\mathrm{C}(2)-\mathrm{C}(3) \quad 108.2(8) \\
& \mathrm{C}(2)-\mathrm{C}(3)-\mathrm{C}(4) \quad 105.9(9) \\
& N(21)-C(4)-C(3) \quad 110.3(7) \\
& \mathrm{N}(21)-\mathrm{C}(4)-\mathrm{C}(5) \quad 126.7(8) \\
& \mathrm{C}(3)-\mathrm{C}(4)-\mathrm{C}(5) \quad 122.9(8) \\
& \mathrm{C}(4)-\mathrm{C}(5)-\mathrm{C}(21) \quad 118.2(8) \\
& \mathrm{C}(4)-\mathrm{C}(5)-\mathrm{C}(6) \quad 124.0(8) \\
& \mathrm{C}(21)-\mathrm{C}(5)-\mathrm{C}(6) \quad 117.8(7) \\
& \mathrm{C}(5)-\mathrm{C}(21)-\mathrm{C}(22) \quad 118.6(5) \\
& \mathrm{C}(5)-\mathrm{C}(21)-\mathrm{C}(26) \quad 121.3(5) \\
& \mathrm{N}(22)-\mathrm{C}(6)-\mathrm{C}(5) \quad 125.9(7) \\
& \mathrm{N}(22)-\mathrm{C}(6)-\mathrm{C}(7) \quad 109.6(7) \\
& \mathrm{C}(5)-\mathrm{C}(6)-\mathrm{C}(7) \quad 124.1(8) \\
& \mathrm{C}(6)-\mathrm{C}(7)-\mathrm{C}(8) \quad 106.1(8) \\
& C(7)-C(8)-C(9) \quad 108.8(8) \\
& \mathrm{N}(22)-\mathrm{C}(9)-\mathrm{C}(8) \quad 107.3(8) \\
& \mathrm{N}(22)-\mathrm{C}(9)-\mathrm{C}(10) \quad 126.4(7) \\
& \mathrm{C}(8)-\mathrm{C}(9)-\mathrm{C}(10) \quad 126.1(7) \\
& \mathrm{C}(9)-\mathrm{C}(10)-\mathrm{C}(27) \quad 117.5(7) \\
& \mathrm{C}(27)-\mathrm{C}(10)-\mathrm{C}(1 \mathrm{~A}) \quad 114.4(7) \\
& \mathrm{C}(10)-\mathrm{C}(27)-\mathrm{C}(28) \quad 121.5(4) \\
& C(10)-C(27)-C(32) \quad 118.5(4) \\
& S(2)-C(145)-\operatorname{Ru}(2) \quad 174.0(37) \\
& \mathrm{C}(145)-\mathrm{Ru}(2)-\mathrm{O}(2) \quad 170.7(22) \\
& \mathrm{C}(145)-\mathrm{Ru}(2)-\mathrm{N}(121) \quad 91.7(14) \\
& O(2)-\operatorname{Ru}(2)-N(121) \quad 95.5(12) \\
& \mathrm{C}(145)-\operatorname{Ru}(2)-\mathrm{N}(122) \quad 93.7(17) \\
& O(2)-\operatorname{Ru}(2)-N(122) \quad 92.3(14) \\
& \mathrm{N}(121)-\mathrm{Ru}(2)-\mathrm{N}(122) \quad 89.4(3) \\
& \text { Ru }(2)-O(2)-C(146) \quad 116.7(30)
\end{aligned}
$$




$$
\begin{array}{ll}
O(2)-C(146)-C(147) & 111.8(44) \\
R u(2)-N(121)-C(101) & 127.2(7) \\
R u(2)-N(121)-C(104) & 127.0(6) \\
C(101)-N(121)-C(104) & 105.8(7) \\
R u(2)-N(122)-C(106) & 127.2(5) \\
R u(2)-N(122)-C(109) & 126.5(7) \\
C(106)-N(122)-C(109) & 106.3(8) \\
N(121)-C(101)-C(102) & 110.4(9) \\
C(101)-C(102)-C(103) & 106.9(8) \\
C(102)-C(103)-C(104) & 107.5(10) \\
N(121)-C(104)-C(103) & 109.3(9) \\
N(121)-C(104)-C(105) & 126.7(8) \\
C(103)-C(104)-C(105) & 123.9(10) \\
C(104)-C(105)-C(121) & 118.9(8) \\
C(104)-C(105)-C(106) & 123.3(10) \\
C(121)-C(105)-C(106) & 117.8(9) \\
C(105)-C(121)-C(122) & 120.4(5) \\
C(105)-C(121)-C(126) & 119.5(5) \\
N(122)-C(106)-C(105) & 126.3(9) \\
N(122)-C(106)-C(107) & 109.3(8) \\
C(105)-C(106)-C(107) & 124.3(11) \\
C(106)-C(107)-C(108) & 107.8(10) \\
C(107)-C(108)-C(109) & 108.5(8) \\
N(122)-C(109)-C(108) & 108.1(9) \\
N(122)-C(109)-C(110) & 124.1(10) \\
C(108)-C(109)-C(110) & 127.7(8) \\
C(109)-C(110)-C(127) & 113.9(9) \\
C(109)-C(110)-C(10 B) & 127.8(8) \\
C(10)-C(10 B) & 118.3(8) \\
C(127)-C(128) & 118.2(5) \\
C(127)-C(132) & 121.7(5)
\end{array}
$$


Table 5D. Anisotropic displacement coefficients $\left(\mathrm{A}^{2} \times 10^{3}\right)$

\begin{tabular}{|c|c|c|c|c|c|c|}
\hline & $\mathrm{U}_{11}$ & $\mathrm{U}_{22}$ & $\mathrm{U}_{33}$ & $U_{12}$ & $\mathrm{U}_{13}$ & $U_{23}$ \\
\hline$S(1)$ & $34(3)$ & $122(6)$ & $130(6)$ & $-12(4)$ & $-11(3)$ & $-65(5)$ \\
\hline $\mathrm{Ru}(1)$ & $89(1)$ & $23(1)$ & $47(1)$ & $-14(1)$ & $-14(1)$ & $-2(1)$ \\
\hline$N(21)$ & $46(4)$ & $23(3)$ & $55(4)$ & $-5(3)$ & $-19(4)$ & $-2(3)$ \\
\hline$N(22)$ & $49(4)$ & $27(3)$ & $51(4)$ & $-7(3)$ & $-16(3)$ & $1(3)$ \\
\hline$C(1)$ & $43(5)$ & $28(4)$ & $64(6)$ & $-8(4)$ & $-4(4)$ & $-8(4)$ \\
\hline$C(2)$ & $59(6)$ & $36(5)$ & $77(7)$ & $-8(4)$ & $-21(5)$ & $-17(5)$ \\
\hline$C(3)$ & $63(6)$ & $46(5)$ & $64(6)$ & $-8(5)$ & $-17(5)$ & $-21(5)$ \\
\hline$C(4)$ & $49(6)$ & $30(4)$ & $55(6)$ & $-5(4)$ & $-3(5)$ & $-7(4)$ \\
\hline$C(5)$ & $45(5)$ & $47(5)$ & $48(5)$ & $-12(4)$ & $-10(4)$ & $-7(4)$ \\
\hline$C(22)$ & $99(9)$ & $64(7)$ & $63(7)$ & $-23(6)$ & $-30(6)$ & $-14(5)$ \\
\hline$C(23)$ & $135(12)$ & $79(8)$ & $80(9)$ & $-42(8)$ & $-36(8)$ & $-9(7)$ \\
\hline$C(24)$ & $127(11)$ & $105(10)$ & $53(7)$ & $-44(9)$ & $-14(7)$ & $-10(7)$ \\
\hline$c(25)$ & $84(8)$ & $99(9)$ & $54(7)$ & $-35(7)$ & $-9(6)$ & $6(6)$ \\
\hline$c(26)$ & $81(8)$ & $60(6)$ & $51(6)$ & $-24(6)$ & $-8(5)$ & $-4(5)$ \\
\hline$C(21)$ & $60(6)$ & $45(5)$ & $46(5)$ & $-12(4)$ & $-17(5)$ & $-7(4)$ \\
\hline$c(6)$ & $57(6)$ & $39(5)$ & $45(5)$ & $-11(4)$ & $-18(4)$ & $1(4)$ \\
\hline$C(7)$ & $89(8)$ & $46(6)$ & $54(6)$ & $-19(5)$ & $-28(6)$ & $3(4)$ \\
\hline$C(8)$ & $90(8)$ & $36(5)$ & $63(6)$ & $-10(5)$ & $-30(6)$ & $7(4)$ \\
\hline$C(9)$ & $36(5)$ & $32(4)$ & $58(5)$ & $-8(4)$ & $-6(4)$ & $0(4)$ \\
\hline$C(10)$ & $52(6)$ & $25(4)$ & $62(6)$ & $-9(4)$ & $-16(5)$ & $4(4)$ \\
\hline$c(28)$ & $87(8)$ & $35(5)$ & $69(7)$ & $-15(5)$ & $-26(6)$ & $-4(4)$ \\
\hline$C(29)$ & $105(9)$ & $34(5)$ & $79(7)$ & $-18(6)$ & $-29(7)$ & $-7(5)$ \\
\hline
\end{tabular}




\begin{tabular}{|c|c|c|c|c|c|c|}
\hline$C(30)$ & $81(9)$ & $37(6)$ & $142(12)$ & $1(6)$ & $-9(8)$ & $-4(6)$ \\
\hline$C(31)$ & $48(8)$ & $44(7)$ & $352(26)$ & $0(6)$ & $-34(11)$ & $11(11)$ \\
\hline$C(32)$ & $37(7)$ & $38(6)$ & $321(22)$ & $-5(5)$ & $-26(10)$ & $22(9)$ \\
\hline$C(27)$ & $54(6)$ & $29(4)$ & $72(6)$ & $-3(4)$ & $-30(5)$ & $-1(4)$ \\
\hline$S(2)$ & $105(6)$ & $72(4)$ & $83(4)$ & $-46(5)$ & $-22(4)$ & $-3(3)$ \\
\hline$C(145)$ & $35(16)$ & $34(11)$ & $56(18)$ & $-21(11)$ & $4(14)$ & $-9(12)$ \\
\hline $\operatorname{Ru}(2)$ & $83(1)$ & $48(1)$ & $54(1)$ & $16(1)$ & $-22(1)$ & $-16(1)$ \\
\hline$O(2)$ & $84(22)$ & $116(27)$ & $69(18)$ & $-33(18)$ & $3(15)$ & $-3(16)$ \\
\hline$C(146)$ & $311(81)$ & $82(27)$ & $221(53)$ & $5(41)$ & $58(52)$ & $86(32)$ \\
\hline$N(121)$ & $44(5)$ & $44(4)$ & $74(5)$ & $-1(3)$ & $-15(4)$ & $-11(4)$ \\
\hline$N(122)$ & $72(6)$ & $44(4)$ & $47(4)$ & $-9(4)$ & $-8(4)$ & $-13(3)$ \\
\hline$C(101)$ & $71(7)$ & $43(5)$ & $53(6)$ & $-5(5)$ & $-8(5)$ & $-10(4)$ \\
\hline$C(102)$ & $49(6)$ & $59(6)$ & $74(7)$ & $-4(5)$ & $-10(5)$ & $-24(5)$ \\
\hline$C(103)$ & $61(7)$ & $71(7)$ & $73(7)$ & $-7(6)$ & $-15(6)$ & $-29(6)$ \\
\hline$C(104)$ & $55(6)$ & $42(5)$ & $69(6)$ & $-4(4)$ & $-27(5)$ & $-11(4)$ \\
\hline$C(105)$ & $59(6)$ & $44(5)$ & $64(6)$ & $-11(5)$ & $-11(5)$ & $-12(4)$ \\
\hline$C(122)$ & $74(8)$ & $58(6)$ & $95(9)$ & $9(6)$ & $-46(7)$ & $-19(6)$ \\
\hline$C(123)$ & $101(10)$ & $83(9)$ & $111(10)$ & $6(8)$ & $-58(9)$ & $-35(7)$ \\
\hline$C(124)$ & $129(13)$ & $99(11)$ & $150(15)$ & $3(9)$ & $-85(12)$ & $-59(10)$ \\
\hline$C(125)$ & $128(13)$ & $125(13)$ & $178(17)$ & $-51(11)$ & $-71(13)$ & $-40(12)$ \\
\hline$C(126)$ & $117(11)$ & $92(9)$ & $126(12)$ & $-39(9)$ & $-45(10)$ & $-34(8)$ \\
\hline$C(121)$ & $77(8)$ & $50(6)$ & $89(8)$ & $-2(5)$ & $-41(7)$ & $-29(6)$ \\
\hline$C(106)$ & $84(8)$ & $37(5)$ & $58(6)$ & $-26(5)$ & $-21(5)$ & $-6(4)$ \\
\hline$C(107)$ & $76(7)$ & $42(5)$ & $55(6)$ & $-15(5)$ & $-13(5)$ & $-11(4)$ \\
\hline
\end{tabular}




$\begin{array}{lllllll}C(108) & 59(7) & 47(5) & 58(6) & -21(5) & -2(5) & -16(4) \\ C(109) & 72(7) & 52(6) & 52(6) & -25(5) & -3(5) & -13(5) \\ C(110) & 58(6) & 42(5) & 55(6) & -5(5) & -3(5) & -12(4) \\ C(128) & 191(15) & 42(6) & 88(9) & -15(8) & 26(9) & -26(6) \\ C(129) & 205(17) & 62(8) & 90(10) & -8(9) & 19(10) & -23(7) \\ C(130) & 80(9) & 76(8) & 103(10) & 11(7) & -31(7) & -45(7) \\ C(131) & 64(8) & 117(11) & 125(11) & -24(8) & 13(7) & -68(9) \\ C(132) & 51(7) & 101(9) & 129(11) & -38(7) & 29(7) & -72(8) \\ C(127) & 61(7) & 54(6) & 56(6) & -5(5) & -9(5) & -17(5)\end{array}$

The anisotropic displacement exponent takes the form: $-2 \pi^{2}\left(h^{2} a \star^{2} U_{11}+\ldots+2 h k a * b * U_{12}\right)$

Table6D. H-Atom coordinates $\left(\times 10^{4}\right)$ and isotropic displacement coefficients $\left(\mathrm{A}^{2} \times 10^{3}\right)$

$\begin{array}{lrrrl}\mathrm{H}(46 \mathrm{~A}) & 1234 & 222 & 322 & 129 \\ \mathrm{H}(46 \mathrm{~B}) & 1984 & 1258 & 166 & 129 \\ \mathrm{H}(47 \mathrm{~A}) & 824 & 1598 & -818 & 135 \\ \mathrm{H}(47 \mathrm{~B}) & 1670 & 222 & -1027 & 135 \\ \mathrm{H}(47 \mathrm{C}) & 2420 & 1258 & -1183 & 80 \\ \mathrm{H}(2) & 2627 & 4278 & 1132 & 167(59) \\ \mathrm{H}(3) & 2954 & 2684 & 2333 & 42(21) \\ \mathrm{H}(22) & 5307 & 1216 & 2762 & 126(20) \\ \mathrm{H}(23) & 4807 & 1431 & 4120 & 126(20) \\ \mathrm{H}(24) & 3172 & 546 & 5048 & 126(20) \\ \mathrm{H}(25) & 2037 & -555 & 4618 & 126(20) \\ \mathrm{H}(26) & 2538 & -770 & 3260 & 126(20) \\ \mathrm{H}(7) & 5169 & -2337 & 2841 & 43(22) \\ \mathrm{H}(8) & 6201 & -4057 & 1971 & 87(33)\end{array}$




\begin{tabular}{|c|c|c|c|c|}
\hline$H(28)$ & 5185 & -5142 & 670 & $286(45)$ \\
\hline $\mathrm{H}(29)$ & 6218 & -7356 & 861 & $286(45)$ \\
\hline $\mathrm{H}(30)$ & 8610 & -8195 & 781 & $286(45)$ \\
\hline $\mathrm{H}(31)$ & 9968 & -6819 & 509 & $286(45)$ \\
\hline $\mathrm{H}(32)$ & 8934 & -4604 & 317 & $286(45)$ \\
\hline $\mathrm{H}(146)$ & 2502 & 4048 & 5728 & 390 \\
\hline$H(156)$ & 3292 & 3139 & 5074 & 334 \\
\hline $\mathrm{H}(14 \mathrm{~A})$ & 1968 & 2247 & 6088 & 128 \\
\hline$H(14 B)$ & 2728 & 2357 & 6696 & 128 \\
\hline $\mathrm{H}(14 \mathrm{C})$ & 3518 & 1449 & 6042 & 128 \\
\hline$H(102)$ & 41 & 8105 & 5627 & $34(20)$ \\
\hline $\bar{H}(103)$ & 218 & 7354 & 4359 & $74(32)$ \\
\hline$H(122)$ & 2837 & 6941 & 2281 & $194(30)$ \\
\hline $\mathrm{H}(123)$ & 1405 & 7187 & 1424 & $194(30)$ \\
\hline$H(124)$ & -404 & 6263 & 1841 & $194(30)$ \\
\hline $\mathrm{H}(125)$ & -781 & 5094 & 3115 & $194(30)$ \\
\hline$H(126)$ & 652 & 4847 & 3972 & $194(30)$ \\
\hline$H(107)$ & 4210 & 4247 & 2432 & $115(45)$ \\
\hline $\mathrm{H}(108)$ & 6606 & 2907 & 2436 & $49(23)$ \\
\hline $\mathrm{H}(128)$ & 7781 & 538 & 3854 & $253(41)$ \\
\hline$H(129)$ & 9305 & -994 & 3028 & $253(41)$ \\
\hline $\mathrm{H}(130)$ & 10994 & -447 & 1942 & $253(41)$ \\
\hline $\mathrm{H}(131)$ & 11161 & 1633 & 1682 & $253(41)$ \\
\hline $\mathrm{H}(132)$ & 9638 & 3165 & 2507 & $253(41)$ \\
\hline
\end{tabular}




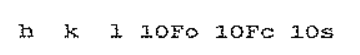

h th 1 IOFO IOFC 305

$1001704-1700$ $\begin{array}{rrrrr}2 & 0 & 0 & 871 & 913 \\ 3 & 0 & 0 & 423 & -459\end{array}$ $400 \quad 322 \quad 319$ $500370-366$ $\begin{array}{rrrrrr}6 & 0 & 0 & 367 & 356 & 5 \\ 7 & 0 & 0 & 169 & -155 & 7\end{array}$ $80078 \quad 37 \quad 10$ $\begin{array}{llllll}9 & 0 & 0 & 166 & -194 & 7\end{array}$ $1000116 \quad 133 \quad 12$ $\begin{array}{rrrrrr}11 & 0 & 0 & 71 & -65 & -17 \\ 12 & 0 & 0 & 77 & 59 & -19\end{array}$ $\begin{array}{llllll}-32 & 1 & 0 & 32 & 21 & -44\end{array}$ $\begin{array}{llllll}-11 & 1 & 0 & 55 & -23 & -29\end{array}$ $\begin{array}{rrllll}-10 & 1 & 0 & 59 & -28 & -23 \\ -9 & 1 & 0 & 53 & -36 & -30\end{array}$ $\begin{array}{rrrrrr}-8 & 1 & 0 & 40 & -19 & -33 \\ -7 & 1 & 0 & 153 & 126 & 8\end{array}$ $\begin{array}{rrrrrr}-7 & 1 & 0 & 153 & 126 & 8 \\ -6 & 1 & 0 & 44 & -6 & -28\end{array}$ $\begin{array}{llllll}-5 & 1 & 0 & 67 & 52 & 13\end{array}$

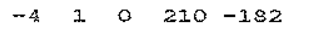
$\begin{array}{llllr}-3 & 1 & 0 & 263 & 248 \\ -2 & 1 & 0 & 1093-1107\end{array}$ $\begin{array}{lllll}-1 & 1 & 0 & 427 & -366\end{array}$ $0100256-169$ $\begin{array}{rrrrr}1 & 1 & 0 & 1564 & 1555\end{array}$ $\begin{array}{rrrrrr}3 & 1 & 0 & 638 & -637 & 5 \\ 4 & 1 & 0 & 202 & 178 & 5\end{array}$ $5 \quad 1 \quad 0 \quad 48 \quad 3-17$ $\begin{array}{rrrrrr}6 & 1 & 0 & 106 & 100 & 10 \\ 7 & 1 & 0 & 93 & -36 & 11\end{array}$ $\begin{array}{rrrrrr}8 & 1 & 0 & 88 & -39 & 13 \\ 9 & 1 & 0 & 55 & -75 & -29\end{array}$ 1010 I $100 \quad-32 \quad 15$ $\begin{array}{llllll}11 & 1 & 0 & 44 & 11 & -34 \\ 12 & 1 & 0 & 70 & 11 & -25\end{array}$ $\begin{array}{rrrrrr}12 & 1 & 0 & 70 & 11 & -25 \\ 13 & 1 & 0 & 60 & -2 & -32 \\ -12 & 2 & 0 & 43 & 23 & -37\end{array}$ $\begin{array}{rrrrrr}-12 & 2 & 0 & 43 & 23 & -37\end{array}$ $\begin{array}{llllll}-10 & 2 & 0 & 52 & 74 & -27\end{array}$ $\begin{array}{rrrrrr}-9 & 2 & 0 & 181 & -217 & 10 \\ -8 & 2 & 0 & 96 & 68 & 14\end{array}$ $\begin{array}{llllll}-7 & 2 & 0 & 103 & -90 & 12\end{array}$ $\begin{array}{lllll}-5 & 2 & 0 & 266 & -275\end{array}$ $\begin{array}{lllll}-4 & 2 & 0 & 519 & 538\end{array}$ $\begin{array}{llllrl}-3 & 2 & 0 & 502 & -491 \\ -2 & 2 & 0 & 818 & 780 & 5\end{array}$

$\begin{array}{rrrrrr}-1 & 2 & 0 & 93 & 40 & 10\end{array}$ $022001091 \quad 985$ 220634649 $320908-949$ $\begin{array}{llllr}4 & 2 & 0 & 839 & 843\end{array}$ 62002480 $\begin{array}{llllll}7 & 2 & 0 & 75 & 37 & 14 \\ 8 & 2 & 0 & 80 & 81 & 25\end{array}$ $\begin{array}{rrrrrr}9 & 2 & 0 & 183 & -179 & 9 \\ 10 & 2 & 0 & 106 & 131 & 15\end{array}$ $112041 \quad-74-35$ $\begin{array}{llllll}12 & 2 & 0 & 13 & 59 & -53\end{array}$ $\begin{array}{llllll}13 & 2 & 0 & 27 & -24 & -47\end{array}$ $\begin{array}{rrrrrr}-11 & 3 & 0 & 41 & 12 & -35 \\ -10 & 3 & 0 & 27 & 4 & -38\end{array}$ $\begin{array}{rrrrrr}-9 & 3 & 0 & 68 & 61 & -20 \\ -8 & 3 & 0 & 38 & -53 & 14\end{array}$ $\begin{array}{rrrrrr}-8 & 3 & 0 & 88 & -53 & 14 \\ -7 & 3 & 0 & 67 & 8 & -16 \\ -6 & 3 & 0 & 92 & -40 & 13\end{array}$ $\begin{array}{llllll}-5 & 3 & 0 & 208 & 224 & 6\end{array}$ $\begin{array}{lllll}-3 & 3 & 0 & 552 & 519\end{array}$ $\begin{array}{lllll}-2 & 3 & 0 & 248 & -241 \\ -1 & 3 & 0 & 228 & -197\end{array}$ 03012121026 $\begin{array}{lllll}1 & 3 & 0 & 258 & 236\end{array}$ $\begin{array}{rrrrrr}2 & 3 & 0 & 1093-1079 & 4 \\ 3 & 3 & 0 & 131 & 25 & -7\end{array}$ $\begin{array}{llllll}3 & 3 & 0 & 131 & 25 & -7 \\ 4 & 3 & 0 & 122 & 14 & -8\end{array}$ $\begin{array}{rrrrrr}5 & 3 & 0 & 156 & -170 & 7 \\ 6 & 3 & 0 & 104 & -73 & 11\end{array}$ $\begin{array}{llllll}7 & 3 & 0 & 104 & -26 & 11\end{array}$ $\begin{array}{llllll}8 & 3 & 0 & 114 & -38 & 10\end{array}$ $930 \quad 113$ 87 13 $\begin{array}{llllll}10 & 3 & 0 & 71 & 24 & -19 \\ 11 . & 3 & 0 & 31 . & 36 & -33\end{array}$ $123005024-23$ $\begin{array}{rrllll}13 & 3 & 0 & 62 & -18 & -22 \\ -11 & 4 & 0 & 33 & -30 & -46\end{array}$

\begin{tabular}{|c|c|c|c|c|c|}
\hline-10 & 4 & 0 & 40 & -8 & -37 \\
\hline-9 & 4 & 0 & 65 & -97 & $-2 I$ \\
\hline-8 & 4 & 0 & 13.8 & 212 & 13 \\
\hline-7 & 4 & 0 & 90 & -81 & 25 \\
\hline-6 & 4 & 0 & 310 & 313 & 6 \\
\hline-5 & 4 & 0 & 369 & -381 & 5 \\
\hline-4 & 4 & 0 & 233 & 224 & 6 \\
\hline-3 & 4 & 0 & 326 & -124 & 8 \\
\hline-2 & 4 & 0 & 186 & 187 & 6 \\
\hline-1 & 4 & 0 & 447 & -386 & 6 \\
\hline 0 & 4 & 0 & 1027 & 895 & 5 \\
\hline 1 & 4 & 0 & 308 & -282 & 5 \\
\hline 2 & 4 & 0 & 630 & 623 & 5 \\
\hline 3 & 4 & 0 & 511 & -490 & 5 \\
\hline 4 & 4 & 0 & 296 & 285 & 5 \\
\hline 5 & 4 & 0 & 307 & -302 & 5 \\
\hline 6 & 4 & 0 & 267 & 300 & 6 \\
\hline 7 & 4 & 0 & 210 & $-22 I$ & 7 \\
\hline 8 & 4 & 0 & 56 & -25 & -25 \\
\hline 9 & 4 & 0 & 152 & -146 & 10 \\
\hline 10 & 4 & 0 & 72 & 39 & -19 \\
\hline 11 & 4 & 0 & 33 & 10 & -38 \\
\hline 2.2 & $A$ & 0 & 35 & 41 & -42 \\
\hline 13 & 4 & 0 & 62 & -3 & -32 \\
\hline-10 & 5 & 0 & 59 & 24 & -32 \\
\hline-9 & 5 & 0 & 35 & 16 & -38 \\
\hline-8 & 5 & 0 & 75 & -111 & -18 \\
\hline-7 & 5 & 0 & 95 & 83 & 15 \\
\hline-6 & 5 & a & 39 & 32 & -31 \\
\hline-5 & 5 & 0 & 91 & -73 & 13 \\
\hline-4 & 5 & 0 & 211 & 100 & 11. \\
\hline-3 & 5 & 0 & 92 & 83 & 11 \\
\hline-2 & 5 & 0 & 131 & -153 & 8 \\
\hline-1 & 5 & 0 & 289 & 255 & 5 \\
\hline 0 & 5 & 0 & 178 & -148 & 6 \\
\hline 1 & 5 & 0 & 360 & 13.5 & 7 \\
\hline 2 & 5 & 0 & 168 & 165 & 6 \\
\hline 3 & 5 & o & 133 & 102 & 8 \\
\hline 4 & 5 & 0 & 225 & 230 & 6 \\
\hline 5 & 5 & 0 & 48 & 60 & -18 \\
\hline 6 & 5 & 0 & 101 & 32 & 10 \\
\hline 7 & 5 & 0 & 141 & 88 & 9 \\
\hline 8 & 5 & 0 & 76 & 91 & -16 \\
\hline 9 & 5 & 0 & 103 & -73 & 13 \\
\hline 10 & 5 & 0 & 80 & -34 & -16 \\
\hline 11 & 5 & 0 & 65 & -10 & -26 \\
\hline 32 & 5 & $\circ$ & 25 & -24 & -47 \\
\hline-9 & 6 & 0 & 108 & -61 & 26 \\
\hline-8 & 6 & o & 38 & 81 & -40 \\
\hline-7 & G & 0 & 54 & -66 & -30 \\
\hline-6 & 6 & 0 & 161 & 238 & 10 \\
\hline-5 & 6 & 0 & 134 & -133 & 10 \\
\hline-4 & 6 & 0 & 134 & 135 & 10 \\
\hline-3 & 6 & 0 & 339 & -342 & 6 \\
\hline-2 & 6 & 0 & 522 & 504 & 7 \\
\hline-1 & 6 & 0 & 526 & -512 & 7 \\
\hline 0 & 6 & 0 & 681 & 625 & 6 \\
\hline I. & 6 & 0 & 282 & -286 & 5 \\
\hline 2 & 6 & $\circ$ & 149 & 227 & 6 \\
\hline 3 & 6 & 0 & 220 & -233 & 6 \\
\hline * & 6 & o & 276 & 257 & 6 \\
\hline 5 & 5 & 0 & 183 & -171 & 7 \\
\hline 5 & 6 & 0 & 141 & 98 & 8 \\
\hline 7 & 6 & 0 & 102 & -61 & 12 \\
\hline 8 & 6 & 0 & 139 & 354 & 22 \\
\hline 9 & 6 & 0 & 109 & -106 & 35 \\
\hline 10 & 5 & 0 & 130 & 233 & 23 \\
\hline 21 & 6 & 0 & 61 & -49 & -30 \\
\hline 12 & 6 & 0 & 59 & 28 & -29 \\
\hline 13 & 6 & 0 & 46 & -16 & -40 \\
\hline-9 & 7 & 0 & 98 & -41 & 128 \\
\hline$-\varepsilon$ & 7 & 0 & 41 & -12 & -38 \\
\hline-7 & 7 & 0 & 45 & 38 & -37 \\
\hline-6 & 7 & 0 & 78 & -13 & -17 \\
\hline-5 & 7 & 0 & 56 & 94 & -28 \\
\hline-4 & 7 & 0 & 57 & 66 & -27 \\
\hline-3 & 7 & 0 & 46 & 29 & -33 \\
\hline-2 & 7 & 0 & 13 & 106 & -50 \\
\hline-1 & 7 & 0 & 212 & -206 & 7 \\
\hline 0 & 7 & 0 & 242 & 211 & 6 \\
\hline 1 & 7 & 0 & 66 & -91 & -16 \\
\hline 2 & 7 & 0 & 65 & 46 & -15 \\
\hline 3 & 7 & 0 & 38 & -14 & -28 \\
\hline 4 & 7 & 0 & 21 t & 3.07 & IO \\
\hline 5 & 7 & 0 & 74 & -120 & -15 \\
\hline 6 & 7 & o & 51 & 3 & -29 \\
\hline 7 & 7 & 0 & 97 & -73 & 14 \\
\hline 8 & 7 & 0 & 52 & -8 & -31 \\
\hline 9 & 7 & 0 & 60 & -39 & -29 \\
\hline
\end{tabular}

b d 1 l.OFO IOFC IOS

$\begin{array}{rrrrrr}7 & 12 & 0 & 49 & -95 & -36 \\ 8 & 12 & 0 & 75 & 15 & -22\end{array}$ $\begin{array}{llllll}13 & 7 & 0 & 36 & 24 & -41 \\ -6 & 0 & 47 & -7 & -37\end{array}$ $\begin{array}{rrrrrr}-8 & 8 & 0 & 38 & 49 & -42 \\ -7 & 8 & 0 & 104 & -135 & 19\end{array}$ $\begin{array}{rlllrl}-6 & 8 & 0 & 172 & 190 & 11\end{array}$ $\begin{array}{llllll}-5 & 8 & 0 & 162 & -172 & 11 \\ -4 & 8 & 0 & 138 & 125 & 12\end{array}$

$\begin{array}{llllll}-3 & 8 & 0 & 26 & -50 & -143\end{array}$ $\begin{array}{lllll}-2 & 8 & 0 & 395 & 405\end{array}$

$\begin{array}{llllll}-1 & 8 & 0 & 385 & -366\end{array}$

$\begin{array}{rrrrr}0 & 8 & 0 & 516 & 518 \\ 1 & 8 & 0 & 414 & -408\end{array}$

$\begin{array}{llllll}2 & 8 & 0 & 428 & 404 & 6\end{array}$

$\begin{array}{rrrrrr}3 & 8 & 0 & 492 & -484 & 7 \\ 4 & 8 & 0 & 266 & 265 & 7\end{array}$

$\begin{array}{rrrrrr}5 & 8 & 0 & 116 & -102 & 11 \\ 6 & 8 & 0 & 76 & -6 & -16\end{array}$

$\begin{array}{llllll}7 & 8 & 0 & 109 & -72 & 12\end{array}$

$8 \quad 8 \quad 0 \quad 81 \quad 55-19$

$\begin{array}{llllll}9 & 0 & 137 & -152 & 13\end{array}$

$\begin{array}{lllll}10 & 8 & 41 & 62 & -37\end{array}$

$\begin{array}{llllll}11 & 8 & 0 & 68 & -57 & -22\end{array}$

$\begin{array}{rrrrrr}12 & 8 & 0 & 32 & 66 & -39 \\ -7 & 9 & 0 & 118 & 27 & 23\end{array}$

$\begin{array}{llllll}-6 & 9 & 0 & 63 & -19 & -29\end{array}$

$\begin{array}{llllll}-5 & 9 & 0 & 27 & -5 & -38 \\ -4 & 9 & 0 & 47 & -22 & -34\end{array}$

$\begin{array}{llllll}-3 & 9 & 0 & 70 & 22 & -18\end{array}$

$\begin{array}{llllll}-2 & 9 & 0 & 34 & 27 & -37\end{array}$

$\begin{array}{rrrrrr}-1 & 9 & 0 & 61 & -54 & -28 \\ 0 & 9 & 0 & 257 & 248 & 7\end{array}$

$\begin{array}{rrrrrr}0 & 9 & 0 & 257 & 248 & 7 \\ 1 & 9 & 0 & 310 & -319 & 7\end{array}$

$\begin{array}{llllll}2 & 9 & 0 & 297 & 291 & 7\end{array}$

$\begin{array}{rrrrrr}3 & 9 & 0 & 162 & -145 & 7 \\ 4 & 9 & 0 & 174 & .164 & 8\end{array}$

$\begin{array}{rrrrrr}4 & 9 & 0 & 174 & 164 & 8 \\ 5 & 9 & 0 & 100 & -102 & 14\end{array}$

$\begin{array}{rrrrrr}6 & 9 & 0 & 81 & 64 & -18 \\ 7 & 9 & 0 & 121 & -56 & 13\end{array}$

$\begin{array}{llllll}8 & 9 & 0 & 57 & 10 & -29\end{array}$

$990 \quad 61 \quad-45-24$

$\begin{array}{rlllrl}10 & 9 & 0 & 79 & 1 & -20 \\ 11 & 9 & 0 & 31 & -12 & -37\end{array}$

$1290 \quad 59 \quad-1-34$

$\begin{array}{llllll}-6 & 10 & 0 & 124 & 155 & 18\end{array}$

$\begin{array}{llllll}-5 & 10 & 0 & 61 & -117 & -35\end{array}$

$\begin{array}{llllll}-4 & 10 & 0 & 153 & 147 & 12\end{array}$

$\begin{array}{llllll}-3 & 10 & 0 & 179 & -181 & 11\end{array}$

$\begin{array}{rrrrrr}-2 & 10 & 0 & 184 & 207 & 11 \\ -1 & 10 & 0 & 120 & -119 & 13\end{array}$

$010 \circ 170 \quad 166 \quad 11$

$\begin{array}{llllll}1 & 10 & 0 & 179 & -207 & 10\end{array}$

$210 \quad 0 \quad 198 \quad 185 \quad 10$

$\begin{array}{llllrr}310 & 0 & 448 & -446 & 6 \\ 4 & 10 & 0 & 368 & 367 & 7\end{array}$

$5100 \quad 181-178 \quad 10$

G $10 \quad 0 \quad 125 \quad 136 \quad 13$

$\begin{array}{lllrrrr}7 & 10 & 0 & 39 & -47 & -40\end{array}$

$\begin{array}{rrrrrr}8 & 10 & 0 & 81 & 77 & -18 \\ 9 & 10 & 0 & 104 & -85 & 15\end{array}$

$1030 \quad 0 \quad 74 \quad 52-20$

$\begin{array}{llllll}11 & 10 & 0 & 74 & -29 & -29\end{array}$

$\begin{array}{llllll}12 & 10 & 0 & 61 & 44 & -34 \\ -5 & 11 & 0 & 27 & 10 & -49\end{array}$

$\begin{array}{llllll}-5 & 11 & 0 & 27 & 10 & -49 \\ -4 & 11 & 0 & 66 & -22 & -24\end{array}$

$\begin{array}{llllll}-3 & 11 & 0 & 87 & 41 & -19\end{array}$

$\begin{array}{llllll}-2 & 11 & 0 & 49 & 25 & -34\end{array}$

$\begin{array}{rrrrrr}-1 & 11 & 0 & 34 & -30 & -41 \\ 0 & 11 & 0 & 92 & 73 & 36\end{array}$

$\begin{array}{rrrrrr}0 & 11 & 0 & 92 & 73 & 16 \\ 1 & 11 & 0 & 56 & 6 & -24\end{array}$

$\begin{array}{llllll}2 & 11 & 0 & 48 & 38 & -33\end{array}$

$\begin{array}{llllll}311 & 0 & 116 & -153 & 14\end{array}$

$\begin{array}{rrrrrr}4 & 11 & 0 & 84 & 99 & 15 \\ 5 & 11 & 0 & 61 & -92 & -31\end{array}$

$\begin{array}{rrrrrr}5 & 11 & 0 & 61 & -92 & -31 \\ 6 & 11 & 0 & 43 & 25 & -37\end{array}$

$\begin{array}{llllll}7 & 11 & 0 & 41 & -3 & -38 \\ 8 & 11 & 0 & 7 I & 18 & -23\end{array}$

$911090 \quad 1.18$

1011 o 41 30 -39

$111100404-1518$

$\begin{array}{rrllll}-3 & 12 & 0 & 161 & -147 & 12 \\ -2 & 12 & 0 & 152 & 165 & 14\end{array}$

$\begin{array}{llllll}-1 & 12 & 0 & 142 & -159 & 15\end{array}$

$0220 \quad 183 \quad 191 \quad 11$

$112 \circ 128-148 \quad 15$

212013410714

$\begin{array}{rrrrrr}3 & 12 & 0 & 113 & -115 & 16 \\ 4 & 12 & 0 & 11 & 71 & -45\end{array}$

$\begin{array}{llllll}5 & 12 & 0 & 12 & -87 & -49 \\ 6 & 12 & 0 & 90 & 100 & -19\end{array}$
912 o $55 \quad-23-36$

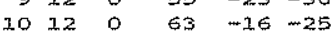

$\begin{array}{llllll}-2 & 13 & 0 & 81 & -11 & -28\end{array}$

$\begin{array}{llllll}-1 & 13 & 0 & 53 & 2 & -30\end{array}$

$\begin{array}{rrrrrr}0 & 13 & 0 & 46 & 48 & -40 \\ 1 & 13 & 0 & 41 & -91 & -43\end{array}$

213010510519

$\begin{array}{llllll}3 & 13 & 0 & 109 & -92 & 17\end{array}$

$4130114 \quad 94 \quad 15$

$\begin{array}{rrrrrr}5 & 13 & 0 & 32 & -57 & -41 \\ 6 & 13 & 0 & 53 & 28 & -35\end{array}$

$\begin{array}{llllll}7 & 13 & 0 & 90 & -13 & -26\end{array}$

$\begin{array}{lllll}13 & 0 & 62 & 23 & -36\end{array}$

$\begin{array}{llllll}13 & 0 & 14 & -35 & -58\end{array}$

$1140 \quad 80 \quad-70 \quad-31$

$\begin{array}{llllll}2 & 14 & 0 & 93 & 68 & -20\end{array}$

$\begin{array}{llllll}3 & 14 & 0 & 172 & -178 & 14\end{array}$

$41400141 \quad 13614$

$\begin{array}{llllll}5 & 14 & 0 & 42 & -51 & -38\end{array}$

$61 \%$ o $66 \quad 51-32$

$\begin{array}{rrrrrr}714 & 0 & 96 & -48 & -19 \\ -6 & -14 & 1 & 123 & 70 & 15\end{array}$

$\begin{array}{llrrr}-5-14 & 1 & 85 & -58 & -18\end{array}$

$\begin{array}{lllll}-4-14 & 1 & 82 & 89 & -22\end{array}$

$\begin{array}{lllll}-3-14 & 1 & 70 & -66 & -23\end{array}$

$\begin{array}{lllrr}-2-14 & 1 & 79 & 9 & -28 \\ -1-14 & 1 & 28 & -46 & -52\end{array}$

$\begin{array}{lllll}-8-13 & 1 & 56 & 14 & -36\end{array}$

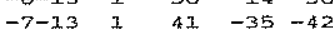

$-6-131$ 1. 68 $88-31$

$\begin{array}{lllrl}-5-13 & 1 & 84 & -105 & -19 \\ -4-13 & 1 & 74 & 82 & -20\end{array}$

$\begin{array}{lllll}-3-13 & 1 & 35 & -73 & -44\end{array}$

$\begin{array}{lllll}-2-13 & 1 & 121 & 147 & 17\end{array}$

$-1-131131-186 \quad 16$

$0-131111 \quad 121 \quad 16$

$\begin{array}{lllll}1-13 & 1 & 181 & -136 & 12\end{array}$

$\begin{array}{lllll}-10-12 & 1 & 61 & 31 & -27\end{array}$

$\begin{array}{rrrrr}-10-12 & 1 & 61 & 31 & -27 \\ -9-12 & \mathrm{l} & 50 & -24 & -29\end{array}$

$\begin{array}{llll}-8-12 & 1 & 51 & 3-38\end{array}$

$\begin{array}{lllll}-7-12 & 1 & 55 & -19 & -34\end{array}$

$\begin{array}{lllll}-6-12 & 1 & 47 & 35 & -34\end{array}$

$\begin{array}{lllll}-5-12 & 1 & 52 & -15 & -31 \\ -4-12 & 1 & 59 & 15 & -25\end{array}$

$\begin{array}{lllll}-4-12 & 1 & 59 & 15 & -25 \\ -3-12 & 1 & 56 & -64 & -30\end{array}$

$\begin{array}{rrrrr}-2-12 & 1 & 48 & 43 & -36 \\ -1-12 & 1 & 34 & -39 & -41\end{array}$

$\begin{array}{lllll}0-12 & 1 & 109 & 97 & 16\end{array}$

$\begin{array}{lllll}1-12 & 1 & 80 & -91 & -21\end{array}$

$\begin{array}{lllll}2-12 & 1 & 42 & 18 & -39\end{array}$

$\begin{array}{rrrrr}3-12 & 1 & 33 & -1 & -49\end{array}$

$\begin{array}{rrrrr}-11-11 & 1 & 79 & -17 & -29 \\ -10-11 & 1 & 90 & 27 & -19\end{array}$

$\begin{array}{lllll}-9-11 & 1 & 80 & -39 & -22\end{array}$

$\begin{array}{lllll}-8-11 & 1 & 54 & 52 & -36\end{array}$

$\begin{array}{lllll}-7-11 & 1 & 62 & -24 & -29\end{array}$

$\begin{array}{rrrrr}-6-11 & 1 & 99 & 65 & 15 \\ -5-11 & 1 & 11 & -17 & -46\end{array}$

$-4-11+51 \quad 85-27$

$\begin{array}{lllll}-3-11 & 1 & 128 & -3.51 & 12\end{array}$

$-2-11 \quad 1$ 85 90-18

$\begin{array}{rrrrr}-1-11 & 1 & 181 & -176 & 10 \\ 0-11 & 1 & 145 & 153 & 13\end{array}$

$\begin{array}{lllll}1-11 & 1 & 105 & -86 & 15\end{array}$

$2-11 \quad 1 \quad 131 \quad 125 \quad 14$

$3-13$ I $362-181$ 12

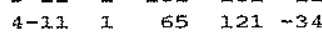

$\begin{array}{rrrrr}5-12 & 2 & 92 & -76 & -20\end{array}$

$\begin{array}{llll}-10-10 & 7 & 75 & 8\end{array}-22$

$\begin{array}{lllll}-9-10 & 1 & 57 & -34 & -30\end{array}$

$\begin{array}{lllll}-8-10 & 1 & 57 & -14 & -30\end{array}$

$\begin{array}{rrrrr}-7-10 & 1 & 92 & -92 & 16 \\ -6-10 & 1 & 57 & 38 & -29\end{array}$

$\begin{array}{lllll}-5-10 & 1 & 50 & -35 & -29\end{array}$

$\begin{array}{lllll}-4-10 & 1 & 73 & 96 & -17\end{array}$

$\begin{array}{lllll}-3-10 & 3 & 43 & 43 & -32\end{array}$

$\begin{array}{lllll}-2-10 & 1 & 63 & 46 & -21\end{array}$

$\begin{array}{lllll}-1-10 & 2 & 100 & -87 & 35\end{array}$

$1-10 \quad 1 \quad 69 \quad 10-25$

$\begin{array}{rrrrr}3-10 & 1 & 32 & 9 & -33\end{array}$

$\begin{array}{rrrrr}3-10 & 1 & 32 & 9 & -33 \\ 4-10 & 1 & 92 & -44 & 14\end{array}$

$\begin{array}{rrrrr}5-10 & 1 & 41 & 2 & -37\end{array}$

$\begin{array}{lllll}6-10 & 1 & 67 & 8 & -33\end{array}$

$\begin{array}{rrrrrr}-12 & -9 & 1 & 47 & 49 & -46 \\ -11 & -9 & 1 & 26 & -75 & -48\end{array}$ k 1 IOFO $10 F C$ IOS

$\begin{array}{llllll}-10 & -9 & 2 & 66 & 92 & -29\end{array}$

$\begin{array}{rrrrrr}-9 & -9 & 1 & 106 & -97 & 15\end{array}$

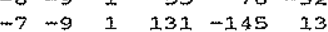

$\begin{array}{llllll}-6 & -9 & 1 & 156 & 136 & 10\end{array}$

$\begin{array}{rrrrrr}-5 & -9 & 1 & 228 & -214 & 7 \\ -4 & -9 & 1 & 58 & 46 & -24\end{array}$

$\begin{array}{rrrrrr}-4 & -9 & 1 & 58 & 46 & -24 \\ -3 & -9 & 1 & 161 & -268 & 8\end{array}$

$\begin{array}{llllll}-2 & -9 & 1 & 339 & 356 & 6\end{array}$

$\begin{array}{llllll}1 & -9 & 1 & 318 & -316\end{array}$

$\begin{array}{llllll}0 & -9 & 1 & 362 & 384 & 6 \\ 1 & -9 & 1 & 326 & -361 & 7\end{array}$

$2-9 \pm 110118 \quad 14$

$\begin{array}{llllll}3 & -9 & I & 35 & -42 & -37\end{array}$

$\begin{array}{llllll}4 & -9 & 1 & 129 & 134 & 13\end{array}$

$\begin{array}{rrrrrr}5 & -9 & 1 & 108 & -109 & 15 \\ 6 & -9 & 1 & 110 & 87 & 16\end{array}$

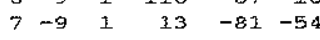

$\begin{array}{llllll}-11 & -8 & 1 & 76 & -9 & -21\end{array}$

$\begin{array}{llllll}10 & -8 & 2 & 94 & 60 & 26\end{array}$

$\begin{array}{llllll}-9 & -8 & 1 & 28 & -46 & -43\end{array}$

$\begin{array}{llllll}-8 & -8 & 1 & 90 & 45 & 17\end{array}$

$\begin{array}{rrrrrr}-7 & -8 & 1 & 37 & -39 & 17 \\ -6 & -8 & 1 & 152 & 158 & 9\end{array}$

$\begin{array}{llllll}-5 & -8 & 1 & 138 & -1.48 & 10\end{array}$

$\begin{array}{lllll}-4 & -8 & 1 & 242 & 243\end{array}$

$\begin{array}{llllll}-3 & -8 & 1 & 349 & -365 & 5\end{array}$

$\begin{array}{rrrrrr}-2 & -8 & 1 & 131 & 132 & 10 \\ -1 & -8 & 1 & 134 & -300 & 13\end{array}$

$\begin{array}{lllll}0 & -8 & 1 & 149 & 148\end{array}$

$\begin{array}{llllll}1 & -8 & 1 & 89 & -67 & 14\end{array}$

$\begin{array}{llllll}2 & -8 & 1 & 138 & 129 & 10\end{array}$

$\begin{array}{rrrrrr}3 & -8 & 1 & 110 & -128 & 14 \\ 4 & -8 & 1 & 11 & 42 & -45\end{array}$

$\begin{array}{llllll}5-8 & 3 & 40 & 65 & -37\end{array}$

$\begin{array}{llllll}6 & -8 & 1 & 92 & -34 & 16\end{array}$

$\begin{array}{llllll}7 & -8 & 1 & 41 & 10 & -37\end{array}$

$\begin{array}{rrrrrr}8 & -8 & 1 & 112 & -30 & 16 \\ -12 & -7 & 1 & 85 & 46 & -19\end{array}$

$\begin{array}{llllll}-11 & -7 & 1 & 61 & -80 & -24\end{array}$

$\begin{array}{llllll}-10 & -7 & 1 & 66 & 63 & -28\end{array}$

$\begin{array}{llllll}-9 & -7 & 1 & 130 & -120 & 13\end{array}$

$\begin{array}{llllll}-8 & -7 & 1 & 193 & 209 & 10\end{array}$

$\begin{array}{rrrrr}-7 & -7 & 1 & 166 & -152 \\ -6 & -7 & 1 & 201 & 209\end{array}$

$\begin{array}{llllll}-5 & -7 & 1 & 108 & -58 & 10\end{array}$

$\begin{array}{llllll}-4 & -7 & 1 & 193 & 196 & 7\end{array}$

$\begin{array}{lllll}-3 & -7 & 1 & 194 & -273\end{array}$

$\begin{array}{rrrrr}-2 & -7 & 1 & 416 & 398 \\ -1 & -7 & 1 & 568 & -565\end{array}$

$0-71616589 ?$

$\begin{array}{lllllll}1 & -7 & 1 & 731 & -711\end{array}$

$\begin{array}{llllll}2 & -7 & 1 & 475 & 486 & 7\end{array}$

$\begin{array}{rrrrrr}3 & -7 & 1 & 470 & -477 & 7 \\ 4 & -7 & 1 & 211 & 208 & 8\end{array}$

$\begin{array}{lllll}5-7 & 1 & 11 & -55 & -45\end{array}$

$\begin{array}{llllll}6 & -7 & 1 & 165 & 172 & 1.1\end{array}$

$\begin{array}{rrrrrr}7 & -7 & 1 & 68 & -84 & -23 \\ 8 & -7 & 1 & 73 & 50 & -21\end{array}$

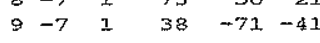

$\begin{array}{llllll}-13 & -6 & 1 & 91 & 9 & -19\end{array}$

$\begin{array}{rrrrrr}-12 & -6 & 1 & 61 & 23 & -23 \\ -11 & -6 & 1 & 51 & 0 & -30\end{array}$

$\begin{array}{rrrrrr}-11 & -6 & 1 & 51 & 0 & -30 \\ -10 & -6 & 1 & 83 & 66 & -18\end{array}$

$\begin{array}{rrrrrr}-9 & -6 & 1 & 92 & -96 & 17 \\ -8 & -6 & 1 & 32 & 26 & -39\end{array}$

$\begin{array}{lllllll}-7 & -6 & 1 & 140 & -98 & 10\end{array}$

$\begin{array}{llllll}-6 & -6 & 1 & 200 & 222 & 7\end{array}$

$\begin{array}{llllll}-5 & -6 & 1 & 153 & -135 & 8\end{array}$

$\begin{array}{llllll}-4 & -6 & 1 & 107 & -24 & 10\end{array}$

$\begin{array}{rrrrrr}-3 & -6 & 1 & 174 & 163 & 6 \\ -2 & -6 & 1 & 91 & 102 & 9\end{array}$

$\begin{array}{llllll}-1 & -6 & 1 & 90 & 42 & 10\end{array}$

$\begin{array}{llllll}0 & -6 & 1 & 313 & 274 & 5\end{array}$

$\begin{array}{rrrrrr}1 & -6 & 1 & 81 & -21 & 13 \\ 2 & -6 & 1 & 74 & 61 & 14\end{array}$

$\begin{array}{llllll}3-6 & 1 & 111 & -86 & 11\end{array}$

$\begin{array}{llllll}4 & -6 & 1 & 148 & -1.49 & 10\end{array}$

$\begin{array}{llllll}5 & -6 & 1 & 195 & -199\end{array}$

$\begin{array}{llllll}6 & -6 & 1 & 44 & 22 & -34\end{array}$

$\begin{array}{rrrrrr}7 & -6 & 1 & 106 & 117 & 16 \\ 8 & -6 & 1 & 72 & -55 & -19\end{array}$

$\begin{array}{llllll}9 & -6 & 2 & 99 & 24 & 16\end{array}$

$\begin{array}{llllll}10 & -6 & 1 & 89 & -21 & -19\end{array}$

$\begin{array}{llllll}-13 & -5 & 2 & 60 & -16 & -33\end{array}$

$\begin{array}{llllll}-22 & -5 & 1 & 40 & 20 & -36\end{array}$

$\begin{array}{rrrrrr}-11 & -5 & 1 & 37 & -38 & -38 \\ -10 & -5 & 1 & 53 & 27 & -32\end{array}$

$\begin{array}{llllrl}-9 & -5 & 1 & 30 & 8 & -39\end{array}$ 
$\mathrm{h} k$ l 1OFO $10 \mathrm{FC}$ 10s

$\begin{array}{rrrrrr}-7 & -5 & 1 & 235 & -238 & 7 \\ -6 & -5 & 1 & 367 & 377 & 5 \\ -5 & -5 & 1 & 357 & -370 & 5 \\ -4 & -5 & 1 & 275 & 307 & 5 \\ -3 & -5 & 1 & 884 & -893 & 5 \\ -2 & -5 & 1 & 327 & 333 & 5 \\ -1 & -5 & 1 & 218 & -220 & 5 \\ 0 & -5 & 1 & 319 & 316 & 5 \\ 1 & -5 & 1 & 587 & -559 & 6 \\ 2 & -5 & 1 & 911 & 857 & 6 \\ 3 & -5 & 1 & 522 & -546 & 7 \\ 4 & -5 & 1 & 526 & 544 & 7 \\ 5 & -5 & 1 & 214 & -202 & 7 \\ 6 & -5 & 1 & 63 & 72 & -11 \\ 7 & -5 & 1 & 187 & -226 & 10 \\ 8 & -5 & 1 & 83 & 62 & -18 \\ 9 & -5 & 1 & 30 & -41 & -44 \\ 10 & -5 & 1 & 13 & 20 & -54 \\ -13 & -4 & 1 & 57 & 15 & -30 \\ -12 & -4 & 1 & 76 & 16 & -21 \\ -10 & -4 & 1 & 81 & 33 & -17 \\ -9 & -4 & 1 & 120 & -75 & 12 \\ -8 & -4 & 1 & 106 & -76 & 11 \\ -7 & -4 & 1 & 138 & 87 & 9 \\ -6 & -4 & 1 & 87 & -44 & 12 \\ -5 & -4 & 1 & 81 & 65 & 11 \\ -4 & -4 & 1 & 289 & 266 & 5 \\ -3 & -4 & 1 & 120 & 62 & 8 \\ -2 & -4 & 1 & 697 & -673 & 5 \\ -1 & -4 & 1 & 201 & -185 & 5 \\ 0 & -4 & 1 & 170 & 136 & 6 \\ 1 & -4 & 1 & 72 & 27 & 12 \\ 2 & -4 & 1 & 206 & -190 & 6 \\ 3 & -4 & 1 & 54 & 60 & -16 \\ 4 & -4 & 1 & 138 & -116 & 8 \\ 5 & -4 & 1 & 158 & 166 & 8 \\ 6 & -4 & 1 & 217 & 230 & 7 \\ 7 & -4 & 1 & 100 & 75 & 14 \\ 8 & -4 & 1 & 117 & -123 & 13 \\ 0 & -4 & 1 & 62 & 42 & -23\end{array}$

$\begin{array}{llllll}9 & -4 & 1 & 62 & 42 & -23\end{array}$

$\begin{array}{rrrrrr}10 & -4 & 3 & 81 & -58 & -19 \\ 11 & -4 & 1 & 109 & 7 & 22\end{array}$

$\begin{array}{rrrrrr}-13 & -3 & 1 & 50 & -23 & -38 \\ -12 & -3 & 1 & 48 & 44 & -37\end{array}$

$\begin{array}{llllll}-11 & -3 & 1 & 58 & -27 & -23\end{array}$

$\begin{array}{llllll}-10 & -3 & 1 & 27 & 89 & -43\end{array}$

$\begin{array}{rrrrrr}-9 & -3 & 1 & 85 & -60 & 17 \\ -8 & -3 & 1 & 43 & 78 & -31\end{array}$

$\begin{array}{llllll}-7 & -3 & 1 & 165 & -157 & 8\end{array}$

$\begin{array}{lllll}-6 & -3 & 1 & 226 & 210\end{array}$

$\begin{array}{lllll}-5 & -3 & 1 & 322 & -322\end{array}$

$\begin{array}{rrrrr}-4 & -3 & 1 & 163 & 145 \\ -3 & -3 & 1 & 552 & -559\end{array}$

$\begin{array}{lllll}-2 & -3 & 1 & 1247 & 1269\end{array}$

$-1-3 \quad 11315-1227$

$\begin{array}{llll}-3 & 1 & 588 & 527\end{array}$

$\begin{array}{lllll}1 & -3 & 1 & 935 & -889\end{array}$

$\begin{array}{rrrrrr}2 & -3 & 1 & 847 & 806 & 5 \\ 3 & -3 & 1 & 709 & -725 & 5\end{array}$

$\begin{array}{lllll}4 & -3 & 1 & 257 & 134\end{array}$

$\begin{array}{lllll}5 & -3 & 1 & 321 & -353\end{array}$

$\begin{array}{lllll}6 & -3 & 1 & 322 & 308\end{array}$

$\begin{array}{rrrrrr}7 & -3 & 1 & 154 & -169 & 9 \\ 8 & -3 & 1 & 160 & 177 & 11\end{array}$

$\begin{array}{llllll}9 & -3 & 1 & 109 & -124 & 15\end{array}$

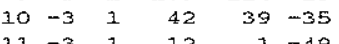

$\begin{array}{llllll}11 & -3 & 1 & 12 & 1 & -49\end{array}$

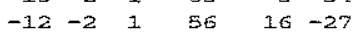

$\begin{array}{rrrrrr}-11 & -2 & 1 & 33 & 10 & -40 \\ -10 & -2 & 1 & 88 & -17 & 16\end{array}$

$\begin{array}{llllll}-9 & -2 & I & 94 & -8 & 15\end{array}$

$\begin{array}{llllll}-8 & -2 & 1 & 110 & -65 & 1.1\end{array}$

$\begin{array}{rrrrrr}-7 & -2 & 1 & 119 & 67 & 10 \\ -6 & -2 & 1 & 56 & 0 & -3.7\end{array}$

$\begin{array}{rrrrrr}-5 & -2 & 1 & 56 & 0 & -1.7 \\ -5 & -2 & 1 & 441 & 460 & 6\end{array}$

$\begin{array}{llllll}-4 & -2 & 1 & 145 & -94 & 7\end{array}$

$\begin{array}{lllll}-2 & -2 & 1 & 699 & -689\end{array}$

$-1-2 \quad 116561615$

$0-21456 \quad 387$

$\begin{array}{llllll}1 & -2 & 1 & 234 & 180 & 5 \\ 2 & -2 & 1 & 424 & 395 & 5\end{array}$

$\begin{array}{rrrrrr}3 & -2 & 1 & 70 & -53 & 12\end{array}$

$\begin{array}{llllll}4 & -2 & 1 & 221 & -208 & 6\end{array}$

$\begin{array}{llllll}5 & -2 & 1 & 66 & 24 & -14\end{array}$

$\begin{array}{llllll}6 & -2 & 1 & 45 & -63 & -29\end{array}$

$\begin{array}{llllll}7 & -2 & 1 & 127 & 83 & 21\end{array}$

$\begin{array}{rrrrrr}8 & -2 & 1 & 129 & -118 & 12 \\ 9 & -2 & 1 & 35 & -4 & -39\end{array}$

h $\mathrm{k}$ I IOFO IOFC IOS

h $\mathrm{k} I$ 1OFO IOFC $10 \mathrm{~S}$

h $k$ I $10 \mathrm{FO}$ LOFC $10 \mathrm{~s}$

$\begin{array}{llllll}10 & -2 & 1 & 13 & -35 & -50\end{array}$

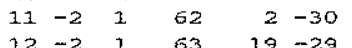

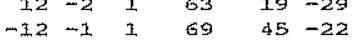

$\begin{array}{rrrrrr}-11 & -1 & 1 & 53 & -87 & -32 \\ -10 & -1 & 1 & 104 & 89 & 13\end{array}$

$\begin{array}{rrrrrr}-10 & -1 & 1 & 104 & 89 & 13 \\ -9 & -1 & 1 & 154 & -143 & 10\end{array}$

$\begin{array}{lllll}-8 & -1 & 1 & 142 & 124\end{array}$

$\begin{array}{lllll}-7 & -1 & 1 & 176 & -183\end{array}$

$\begin{array}{lllll}-6 & -1 & 1 & 257 & 270\end{array}$

$\begin{array}{rrrrr}-5 & -1 & 1 & 326 & -332 \\ -4 & -1 & 1 & 423 & 438\end{array}$

$\begin{array}{lllll}-3 & -1 & 1 & 470 & -426\end{array}$

$\begin{array}{lllll}-2 & -1 & 1 & 161 & 173\end{array}$

$-1.1 \quad 1 \quad 1168-1228$

o $-1 \quad 11451 \quad 1349$

$\begin{array}{rrrrr}1 & -1 & 1 & 522 & -475 \\ 2 & -1 & 1 & 1444 & 1386\end{array}$

$\begin{array}{lllll}3 & -1 & 1 & 832 & -861\end{array}$

$\begin{array}{lllll}4 & -1 & 1 & 529 & 528\end{array}$

$5-1 \quad 1 \quad 260-275$

$\begin{array}{rrrrr}6 & -1 & 1 & 297 & 316 \\ 7 & -1 & 1 & 243 & -242\end{array}$

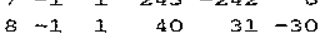

$\begin{array}{llllll}9 & -1 & 1 & 152 & -177 & 11\end{array}$

$\begin{array}{rrrrrr}10 & -1 & 3 & 63 & 57 & -21 \\ 11 & -1 & 1 & 74 & -77 & -19\end{array}$

$\begin{array}{rrrrrr}11 & -1 & 1 & 74 & -77 & -19 \\ 12 & -1 & 1 & 26 & 38 & -48\end{array}$

$\begin{array}{llllll}-12 & 0 & 1 & 89 & 17 & 15\end{array}$

$\begin{array}{llllll}-11 & 0 & 1 & 72 & 26 & -21\end{array}$

$\begin{array}{rrrrrr}-10 & 0 & 1 & 102 & 19 & -23 \\ -9 & 0 & 1 & 118 & -127 & 9\end{array}$

$\begin{array}{rrrrrr}-9 & 0 & 1 & 118 & -127 & 9 \\ -8 & 0 & 1 & 72 & -29 & 12\end{array}$

$\begin{array}{rrrrrr}-7 & 0 & 1 & 143 & 96 & 6\end{array}$

$\begin{array}{lllll}-6 & 0 & 1 & 205 & 173\end{array}$

$\begin{array}{lllll}-5 & 0 & 1 & 286 & 306\end{array}$

$\begin{array}{lllll}-4 & 0 & 1 & 289 & -300\end{array}$

$\begin{array}{lllll}-3 & 0 & 1 & 238 & -147\end{array}$

$\begin{array}{rrrrrr}-2 & 0 & 1 & 100 & -70 & 9\end{array}$

$\begin{array}{llllll}0 & 0 & 1 & 224 & -76 & 20\end{array}$

$\begin{array}{llllll}0 & 0 & 1 & 224 & -76 & 20 \\ 1 & 0 & 1 & 382 & -365 & 2\end{array}$

$\begin{array}{rrrrr}2 & 0 & 1 & 1.92 & 209 \\ 3 & 0 & 1 & 69 & -50\end{array}$

$4 \quad 0 \quad 1 \quad 485-498 \quad 5$

$\begin{array}{rrrrrr}5 & 0 & 1 & 61 & 10 & -13 \\ 6 & 0 & 1 & 257 & 260 & 5\end{array}$

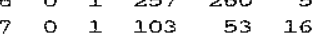

$\begin{array}{rrrrrr}8 & 0 & 1 & 94 & -96 & 9 \\ 9 & 0 & 1 & 87 & -13 & 17\end{array}$

$20 \quad 0 \quad 1 \quad 74 \quad-13 \quad-15$

$\begin{array}{llllll}11 & 0 & 1 & 47 & -17 & -24\end{array}$

$\begin{array}{llllll}12 & 0 & 1 & 94 & 24 & 18\end{array}$

$\begin{array}{rrrrrr}-12 & 1 & 1 & 46 & 25 & -33 \\ -11 & 1 & 1 & 46 & -22 & -34\end{array}$

$\begin{array}{llllll}-10 & 1 & 1 & 116 & 97 & 13\end{array}$

$\begin{array}{llllll}-9 & 1 & 1 & 120 & -121 & 12\end{array}$

$\begin{array}{llllll}-8 & 1 & 1 & 149 & 151 & 10\end{array}$

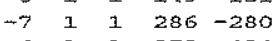

$\begin{array}{rrrrr}-6 & 1 & 1 & 370 & 404 \\ -5 & 1 & 1 & 226 & -206\end{array}$

$\begin{array}{lllll}-4 & 1 & 1 & 759 & 762\end{array}$

$-31.1 .723 .745$

$\begin{array}{lllll}-2 & 1 & 1 & 190 & 198\end{array}$

$\begin{array}{rrrrr}-1 & 1 & 1 & 311 & -284 \\ 0 & 1 & 1 & 1058 & 918\end{array}$

$1111547-1605$

21116981746

$3111567-1610$

$\begin{array}{rrrrr}4 & 1 & 1 & 881 & 879 \\ 5 & 1 & 1 & 511 & -520\end{array}$

611207230

$\begin{array}{lllllll}7 & 1 & 1 & 149 & -128 & 8\end{array}$

$\begin{array}{rrrrrr}8 & 1 & 1 & 70 & 43 & -16 \\ 9 & 1 & 1 & 108 & -73 & 14\end{array}$

$\begin{array}{rrrrrr}9 & 1 & 1 & 108 & -71 & 14 \\ 10 & 1 & 1 & 129 & 115 & 12\end{array}$

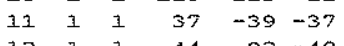

$121144 \quad 92-40$

$\begin{array}{rrrrrr}13 & 1 & 1 & 85 & -41 & -26 \\ -11 & 2 & 1 & 79 & 10 & -18\end{array}$

$\begin{array}{rrrrrr}-11 & 2 & 1 & 79 & 10 & -18 \\ -10 & 2 & 1 & 29 & -4 & -42 \\ -9 & 2 & 1 & 87 & -24 & 16\end{array}$

$\begin{array}{rrrrrr}-9 & 2 & 1 & 87 & -24 & 16 \\ -8 & 2 & 1 & 98 & 75 & 13\end{array}$

$\begin{array}{llllll}-7 & 2 & 1 & 93 & -58 & 13\end{array}$

$\begin{array}{rrrrrr}-6 & 2 & 1 & 123 & 94 & 10 \\ -5 & 2 & 1 & 58 & 3 & -17\end{array}$

$\begin{array}{rrrrrr}-5 & 2 & 1 & 58 & 3 & -17 \\ -4 & 2 & 1 & 303 & -306 & 5\end{array}$

$\begin{array}{llllll}-3 & 2 & 1 & 386 & 383 & 5\end{array}$

$\begin{array}{rrrrrr}-2 & 2 & 1 & 318 & -326 & 5 \\ -1 & 2 & 1 & 208 & 197 & 5\end{array}$ $\begin{array}{llllllllllll}0 & 2 & 1 & 520 & -450 & 4 & -7 & 6 & 1 & 77 & 60 & -20\end{array}$ $\begin{array}{rrrrrr}1 & 2 & 1 & 482 & -440 & 3 \\ 2 & 2 & 1 & 138 & -109 & 7 \\ 3 & 2 & 1 & 978 & -994 & 4 \\ 4 & 2 & 1 & 475 & 460 & 5 \\ 5 & 2 & 1 & 479 & -492 & 6 \\ 6 & 2 & 1 & 213 & 137 & 6 \\ 7 & 2 & 1 & 126 & -99 & 9 \\ 8 & 2 & 1 & 112 & -112 & 11 \\ 9 & 2 & 1 & 41 & -27 & -36\end{array}$

$\begin{array}{rrrrrr}9 & 2 & 1 & 41 & -27 & -36 \\ 10 & 2 & 1 & 27 & -6 & -43\end{array}$

$\begin{array}{llllll}11 & 2 & 1 & 70 & 55 & -43 \\ & 12 & & \end{array}$

$\begin{array}{llllll}12 & 2 & 1 & 43 & 20 & -37\end{array}$

$\begin{array}{rrrrrr}13 & 2 & 1 & 37 & 16 & -43 \\ -11 & 3 & 1 & 65 & 26 & -29\end{array}$

$\begin{array}{rrrrrr}-10 & 3 & 1 & 52 & 42 & -31 \\ -9 & 3 & 1 & 72 & -82 & -21\end{array}$

$\begin{array}{rrrrrr}-9 & 3 & 1 & 72 & -82 & -21 \\ -8 & 3 & 1 & 121 & 135 & 13\end{array}$

$\begin{array}{llllll}-7 & 3 & 2 & 54 & -37 & -26\end{array}$

$\begin{array}{lllll}-6 & 3 & 2 & 300 & 327\end{array}$

$\begin{array}{lllll}-5 & 3 & 1 & 445 & -469\end{array}$

$\begin{array}{rrrrr}-4 & 3 & 1 & 433 & 445 \\ -3 & 3 & 1 & 801 & -809\end{array}$

$\begin{array}{lllll}-2 & 3 & 1 & 573 & 555\end{array}$

$\begin{array}{llllll}-1 & 3 & 1 & 426 & 379 & 5\end{array}$

$\begin{array}{rrrrrr}0 & 3 & 1 & 576 & 542 & 5 \\ 1 & 3 & 1 & 48 & -73 & -14\end{array}$

$\begin{array}{rrrrrr}1 & 3 & 1 & 48 & -73 & -14 \\ 2 & 3 & 1 & 52 & 70 & -16\end{array}$

$\begin{array}{lllll}3 & 3 & 1 & 337 & 345\end{array}$

$\begin{array}{lllll}4 & 3 & 1 & 48 & 487\end{array}$

$\begin{array}{rrrrr}5 & 3 & 1 & 420 & -430 \\ 6 & 3 & 1 & 672 & 694\end{array}$

$\begin{array}{rrrrrr}7 & 3 & 1 & 378 & -408 & 6 \\ 8 & 3 & 1 & 85 & 40 & 13\end{array}$

$\begin{array}{llllll}9 & 3 & 1 & 134 & -142 & I I\end{array}$

$\begin{array}{llllll}10 & 3 & 1 & 94 & 88 & 16\end{array}$

$11311 \quad 13 \quad-61-51$

$\begin{array}{rrrrrr}12 & 3 & 1 & 61 & 47 & -31 \\ 13 & 3 & 1 & 73 & -17 & -22\end{array}$

$\begin{array}{rrrrrr}13 & 3 & 1 & 73 & -17 & -22 \\ -11 & 4 & 1 & 27 & -25 & -49\end{array}$

$\begin{array}{llllll}-10 & 4 & 1 & 32 & -54 & -44\end{array}$

$\begin{array}{rrrrrr}-9 & 4 & 1 & 51 & 17 & -24 \\ -8 & 4 & 1 & 85 & -82 & 15\end{array}$

$\begin{array}{rrrrrr}-8 & 4 & 1 & 85 & -82 & 15 \\ -7 & 4 & 1 & 82 & 11 & 16\end{array}$

$\begin{array}{rrrrrr}-6 & 4 & 1 & 169 & 182 & 8\end{array}$

$\begin{array}{rrrrrr}-5 & 4 & 1 & 32 & 12 & -31 \\ -4 & 4 & 1 & 126 & -88 & 9\end{array}$

$\begin{array}{lllll}-3 & 4 & 1 & 123 & -147\end{array}$

$\begin{array}{llllll}-2 & 4 & 1 & 314 & -293 & 5 \\ -1 & 4 & 1 & 165 & -164 & 6\end{array}$

$04128-11-23$

$\begin{array}{rrrrrr}1 & 4 & 1 & 153 & -154 & 6 \\ 2 & 4 & 1 & 10 & -11 & -40\end{array}$

$\begin{array}{rrrrrr}2 & 4 & 1 & 10 & -11 & -40 \\ 3 & 4 & 1 & 327 & -314 & 5 \\ 4 & 4 & 1 & 88 & 42 & 11\end{array}$

$\begin{array}{rrrrrr}4 & 4 & 1 & 88 & 42 & 11 \\ 5 & 4 & 1 & 237 & 246 & 6\end{array}$

$\begin{array}{rrrrrr}6 & 4 & 1 & 68 & 16 & -14 \\ 7 & 4 & 1 & 272 & 289 & 6\end{array}$

$\begin{array}{rrrrrr}7 & 4 & 1 & 272 & 289 & 6 \\ 8 & 4 & 1 & 110 & -111 & 12\end{array}$

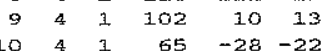

$\begin{array}{llllll}10 & 4 & 1 & 65 & -28 & -22 \\ 11 & 4 & 1 & 37 & -18 & -35\end{array}$

$12 \quad 1 \quad 1 \quad 65 \quad-17-29$

$\begin{array}{llllll}13 & 4 & 1 & 81 & 2 & -19\end{array}$

$\begin{array}{rrrrrr}-10 & 5 & 1 & 89 & 69 & -19 \\ -9 & 5 & 1 & 118 & -110 & 13\end{array}$

$\begin{array}{llllll}-8 & 5 & 1 & 86 & 84 & 17\end{array}$

$\begin{array}{llllll}-7 & 5 & 1 & 104 & -112 & 14\end{array}$

$\begin{array}{llllll}-6 & 5 & 1 & 83 & 68 & 15\end{array}$

$\begin{array}{lllll}-5 & 5 & 1 & 171 & -163\end{array}$

$\begin{array}{lllll}-4 & 5 & 3 & 308 & 334 \\ -3 & 5 & 3 & 472 & -400\end{array}$

$\begin{array}{lllll}-2 & 5 & 1 & 622 & 615\end{array}$

$\begin{array}{lllll}-1 & 5 & 1 & 269 & -258\end{array}$

$\begin{array}{rrrrr}0 & 5 & 2 & 298 & 295 \\ 1 & 5 & 1 & 440 & -433\end{array}$

$\begin{array}{lllll}2 & 5 & 1 & 329 & 323\end{array}$

$\begin{array}{lllll}3 & 5 & 1 & 175 & -172\end{array}$

$451316 \quad 315 \quad 5$

$6 \quad 5 \quad 1 \quad 268 \quad 243 \quad 6$

$\begin{array}{rrrrrr}7 & 5 & 1 & 160 & -174 & 9 \\ 8 & 5 & 1 & 84 & 74 & 15\end{array}$

$95 \quad 1 \quad 74 \quad 30-18$

$\begin{array}{llllll}10 & 5 & 1 & 84 & 81 & -17\end{array}$

$\begin{array}{llllll}11 & 5 & 1 & 42 & 16 & -27 \\ 12 & 5 & 1 & 39 & -7 & -39\end{array}$

$\begin{array}{llllll}13 & 5 & 1 & 37 & -21 & -44\end{array}$

$\begin{array}{llllll}-9 & 6 & 1 & 41 & -7 & -38 \\ -8 & 6 & 1 & 42 & -1 & -33\end{array}$

$\begin{array}{rrrrrrr}-7 & 6 & 1 & 77 & 60 & -20 & -1 \\ -6 & 6 & 1 & 79 & 36 & -18 \\ -5 & 6 & 1 & 48 & -67 & -29 \\ -4 & 6 & 1 & 74 & -39 & -15 \\ -3 & 6 & 1 & 50 & -23 & -24 \\ -2 & 6 & 1 & 41 & 25 & -29 \\ -1 & 6 & 1 & 94 & -78 & 11 \\ 0 & 6 & 1 & 384 & 352 & 6 \\ 1 & 6 & 1 & 88 & -92 & 11 \\ 2 & 6 & 1 & 276 & -262 & 4 \\ 3 & 6 & 1 & 101 & 71 & 10 & \\ 4 & 6 & 1 & 133 & 114 & 9 & 10 \\ 5 & 6 & 1 & 15 & -104 & -26 & 1 \\ 6 & 6 & 1 & 25 & 42 & -38 & 12 \\ 7 & 6 & 1 & 108 & -73 & 12 & -5 \\ 8 & 6 & 1 & 126 & -127 & 11 & -4 \\ 9 & 6 & 1 & 67 & -32 & -25 & -3 \\ 10 & 6 & 1 & 39 & -7 & -36 & -2 \\ 11 & 6 & 1 & 37 & 36 & -39 & -10 \\ 12 & 6 & 1 & 40 & 17 & -40 & \\ 13 & 6 & 1 & 53 & 42 & -38 & \end{array}$

$\begin{array}{llllll}-1 & 10 & 2 & 83 & -43 & -18\end{array}$

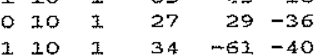

$\begin{array}{llllll}2 & 10 & 3 & 229 & 132 & 13\end{array}$

$\begin{array}{llllll}3 & 10 & 2 & 283 & -304 & 8\end{array}$

$\begin{array}{lllll}40 & 1 & 277 & 272 & 8\end{array}$

$\begin{array}{rrrrrr}5 & 10 & 1 & 171 & -188 & 11 \\ 6 & 10 & 1 & 153 & 168 & 12\end{array}$

$\begin{array}{llllll}7 & 10 & 1 & 117 & -98 & 15\end{array}$

$\begin{array}{lllll}10 & 1 & 88 & 48 & 17\end{array}$

$\begin{array}{llllll}9 & 10 & 1 & 82 & -71 & -1.9\end{array}$

$\begin{array}{llllll}11 & 10 & 1 & 63 & 24 & -31\end{array}$

$\begin{array}{llllrr}12 & 10 & 1 & 59 & 8 & -24\end{array}$

$\begin{array}{llllll}-5 & 11 & 1 & 124 & -127 & 16\end{array}$

$\begin{array}{llllll}-4 & 11 & 1 & 129 & 162 & 17\end{array}$

$\begin{array}{llllll}-3 & 11 & 1 & 177 & -186 & 12\end{array}$

$\begin{array}{rrrrrr}-2 & 11 & 1 & 143 & 160 & 13 \\ -1 & 11 & 1 & 171 & -157 & 13\end{array}$

011219518910

$\begin{array}{llllll}1 & 11 & 1 & 140 & -150 & 13\end{array}$

$\begin{array}{llllll}211 & 1 & 165 & 358 & 11\end{array}$

$\begin{array}{rrrrrr}3 & 11 & 1 & 122 & -127 & 15 \\ 4 & 11 & 1 & 178 & 174 & 10\end{array}$

$\begin{array}{llllll}5 & 11 & 1 & 264 & -282 & 9\end{array}$

$\begin{array}{llllll}6 & 11 & 1 & 216 & 215 & 10\end{array}$

$\begin{array}{llllll}7 & 11 & 1 & 136 & -153 & 14\end{array}$

$\begin{array}{rrrrrr}8 & 11 & 1 & 89 & 108 & -19 \\ 9 & 11 & 1 & 87 & -99 & -19\end{array}$

$\begin{array}{llllll}10 & 11 & 1 & 26 & 66 & -50\end{array}$

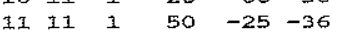

$\begin{array}{llllll}-3 & 12 & 1 & 162 & -42 & 19\end{array}$

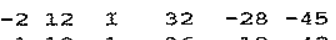

$\begin{array}{rrrrrr}-1 & 12 & 1 & 36 & -1.0 & -42 \\ 0 & 12 & 1 & 35 & 57 & -41\end{array}$

$\begin{array}{llllll}2 & 12 & 1 & 59 & -19 & -32\end{array}$

$\begin{array}{llllll}3 & 12 & 1 & 86 & -13 & -23\end{array}$

$4121110 \quad 113 \quad 15$

$\begin{array}{rrrrrr}5 & 12 & 1 & 97 & -94 & 17 \\ 6 & 12 & 1 & 307 & 120 & 18\end{array}$

$\begin{array}{llllll}7 & 12 & 1 & 99 & -72 & 18\end{array}$

$\begin{array}{llllll}8 & 12 & 1 & 48 & 8 & -33\end{array}$

$\begin{array}{lllll}912 & 1 & 95 & -59 & -19\end{array}$

$\begin{array}{llllll}10 & 12 & 1 & 66 & 40 & -26\end{array}$

$\begin{array}{rrrrrr}-2 & 13 & 1 & 125 & 134 & 16\end{array}$

$\begin{array}{llllll}0 & 13 & 1 & 132 & 128 & 15\end{array}$

$\begin{array}{llllll}1 & 13 & 1 & 91 & -114 & -20\end{array}$

$\begin{array}{rrrrrr}2 & 13 & 1 & 132 & 124 & 15 \\ 3 & 13 & 1 & 50 & -108 & -37\end{array}$

$\begin{array}{llllll}4 & 13 & 1 & 59 & 62 & -29\end{array}$

$\begin{array}{llllll}5 & 1.3 & 1 & 30 & -63 & -43\end{array}$

$\begin{array}{llllll}6 & 13 & 1 & 81 & 73 & -22\end{array}$

$\begin{array}{llllll}7 & 13 & 1 & 32 & -39 & -48\end{array}$

$\begin{array}{llllll}8 & 13 & 1 & 89 & 51 & -21 \\ 9 & 13 & 1 & 14 & -28 & -55\end{array}$

$\begin{array}{llllll}9 & 13 & 1 & 14 & -28 & -55 \\ 1 & 14 & 1 & 65 & -44 & -32\end{array}$

$\begin{array}{llllll}2 & 14 & 1 & 72 & 33 & -31\end{array}$

$\begin{array}{llllll}3 & 14 & 1 & 83 & -51 & -30\end{array}$

$\begin{array}{rrrrrr}4 & 14 & 1 & 96 & 81 & -20 \\ 5 & 14 & 1 & 59 & -79 & -30\end{array}$

$\begin{array}{rrrrrr}5 & 14 & 1 & 59 & -79 & -30 \\ 6 & 14 & 1 & 33 & 48 & -49\end{array}$

$\begin{array}{llllll}7 & 14 & 1 & 102 & -32 & -27\end{array}$

$\begin{array}{lllll}-5-14 & 2 & 13 & -73 & -51\end{array}$

$\begin{array}{rrrrr}-4-14 & 2 & 64 & 88 & -24 \\ -3-14 & 2 & 76 & -81 & -25\end{array}$

$\begin{array}{lllll}-2-14 & 2 & 40 & 57 & -38\end{array}$

$\begin{array}{lllll}-8-13 & 2 & 65 & 73 & -34\end{array}$

$\begin{array}{lllll}-7-13 & 2 & 79 & -61 & -23\end{array}$

$\begin{array}{lllll}-6-13 & 2 & 115 & 100 & 16\end{array}$

$\begin{array}{rrrrr}-5-13 & 2 & 85 & -80 & -19 \\ -4-13 & 2 & 64 & 64 & -23\end{array}$

$\begin{array}{lllll}-3-13 & 2 & 53 & -54 & -36\end{array}$

$\begin{array}{lllll}-2-13 & 2 & 68 & 43 & -34\end{array}$

$\begin{array}{lllll}-1-13 & 2 & 14 & -50 & -57\end{array}$

$\begin{array}{lllll}0-13 & 2 & 43 & 72 & -41\end{array}$

$\begin{array}{rrrrr}1-13 & 2 & 65 & -47 & -34 \\ -9-12 & 2 & 65 & -48 & -33\end{array}$

$\begin{array}{lllll}-8-12 & 2 & 32 & 36 & -49\end{array}$

$\begin{array}{lllll}-7-12 & 2 & 108 & -71 & 15\end{array}$

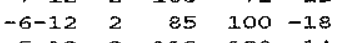

$\begin{array}{llllll}-5-12 & 2 & 110 & -128 & 14\end{array}$

$\begin{array}{lllll}-4-12 & 2 & 163 & 193 & 11\end{array}$

$\begin{array}{lllll}-2-12 \quad 2 & 48 & 122 & -38\end{array}$

$-1-122109-122 \quad 15$

$0-12 \quad 2 \quad 109$ i57 17

$\begin{array}{rrrrr}1-12 & 2 & 121 & -106 & 16 \\ 2-12 & 2 & 83 & 104 & -23\end{array}$

$\begin{array}{lllll}3-12 & 2 & 73 & -106 & -32\end{array}$ 


\section{h $k$ I 10rO 10FC $10 \mathrm{~S}$}

$\begin{array}{lllll}-10-11 \quad 2 & 70 & 12 & -24\end{array}$ $\begin{array}{lllll}-9-11 & 2 & 100 & -25 & 17\end{array}$

$\begin{array}{rrrrr}-8-11 & 2 & 57 & 28 & -31 \\ -7-11 & 2 & 62 & -34 & -23\end{array}$

$\begin{array}{rrrrr}-7-11 & 2 & 62 & -34 & -23 \\ -6-11 & 2 & 68 & 62 & -20\end{array}$

$\begin{array}{lllll}-5-11 & 2 & 60 & -84 & -21 \\ -4-11 & 2 & 11 & 49 & -32\end{array}$

$\begin{array}{lllll}-3-11 & 2 & 81 & -89 & -17\end{array}$

$-2-11 \quad 2 \quad 59 \quad 36-27$

$\begin{array}{ccccc}-1-11 & 2 & 56 & 27 & -31 \\ 0-11 & 2 & 13 & 46 & -53\end{array}$

$\begin{array}{lllll}1-11 . & 2 & 75 & -74 & -19\end{array}$

$\begin{array}{lllll}2-11 & 2 & 120 & 102 & 14\end{array}$

$\begin{array}{lllll}3-11 & 2 & 84 & -68 & -20\end{array}$

$\begin{array}{lllll}4-11 & 2 & 50 & 7 & -39\end{array}$

$\begin{array}{rrrrr}5-11 & 2 & 39 & 27 & -43 \\ -11-10 & 2 & 74 & -29 & -30\end{array}$

$\begin{array}{lllll}-10-10 & 2 & 66 & 65 & -33\end{array}$

$\begin{array}{lllll}-9-10 & 2 & 95 & -79 & 17\end{array}$

$\begin{array}{rrrrr}-5-10 & 2 & 47 & 10 & -29 \\ -7-10 & 2 & 68 & -25 & -27\end{array}$

$\begin{array}{rrrrr}-6-10 & 2 & 93 & 81 & 15 \\ -5-10 & 2 & 329 & -95 & 12\end{array}$

$\begin{array}{lllll}-4-10 & 2 & 205 & 200 & 8\end{array}$

$\begin{array}{lllll}-3-10 & 2 & 126 & -126 & 11\end{array}$

$\begin{array}{rrrrr}-2-10 & 2 & 46 & 96 & -32 \\ -1-10 & 2 & 185 & -201 & 10\end{array}$

$\begin{array}{lllll}0-10 & 2 & 205 & 198 & 9\end{array}$

$\begin{array}{lllll}1-10 & 2 & 155 & -161 & 12\end{array}$

$\begin{array}{lllll}2-10 & 2 & 74 & 86 & -27\end{array}$

$\begin{array}{rrrrr}3-10 & 2 & 48 & -93 & -31 \\ 4-10 & 2 & 150 & 131 & 12\end{array}$

$\begin{array}{rrrrr}4-10 & 2 & 150 & 131 & 12 \\ 5-10 & 2 & 156 & -169 & 11\end{array}$

$\begin{array}{lllll}6-10 & 2 & 109 & 123 & 18\end{array}$

$\begin{array}{llllll}-3.2 & -9 & 2 & 14 & -1 & -55\end{array}$

$\begin{array}{rrrrrr}-11 & -9 & 2 & 87 & -10 & -27 \\ -10 & -9 & 2 & 35 & 21 & -37\end{array}$

$\begin{array}{rrrrrr}-9 & -9 & 2 & 87 & -39 & -18 \\ -8 & -9 & 2 & 64 & 72 & -28\end{array}$

$\begin{array}{llllll}-7 & -9 & 2 & 55 & -45 & -29\end{array}$

$\begin{array}{llllll}-6 & -9 & 2 & 109 & 72 & 14\end{array}$

$\begin{array}{llllll}-5 & -9 & 2 & 110 & -78 & 13\end{array}$

$\begin{array}{rrrrrr}-4 & -9 & 2 & 48 & 22 & -27 \\ -3 & -9 & 2 & 60 & -44 & -26\end{array}$

$\begin{array}{llllll}-2 & -9 & 2 & 103 & 126 & 12\end{array}$

$\begin{array}{llllll}-1 & -9 & 2 & 85 & -29 & -20\end{array}$

$\begin{array}{llllll}0 & -9 & 2 & 36 & 99 & -38\end{array}$

$\begin{array}{rrrrrr}1 & -9 & 2 & 42 & -40 & -35\end{array}$

$\begin{array}{rrrrrr}2 & -9 & 2 & 118 & -134 & 14 \\ 3 & -9 & 2 & 13 & -10 & -53\end{array}$

$\begin{array}{lllllll}4 & -9 & 2 & 73 & -24 & -24\end{array}$

$\begin{array}{llllll}5 & -9 & 2 & 13 & 41 & -51\end{array}$

$\begin{array}{rrrrrr}6 & -9 & 2 & 13 & -26 & -54 \\ 7 & -9 & 2 & 47 & 13 & -42\end{array}$

$\begin{array}{llllll}-12 & -8 & 2 & 57 & 33 & -35\end{array}$

$\begin{array}{rrrrrr}-11 & -8 & 2 & 44 & 4 & -40 \\ -10 & -8 & 2 & 42 & 45 & -35\end{array}$

$\begin{array}{rrrrrr}-10 & -8 & 2 & 42 & 45 & -35 \\ -9 & -8 & 2 & 111 & -116 & 15\end{array}$

$\begin{array}{llllll}-8 & -8 & 2 & 99 & 113 & 16\end{array}$

$\begin{array}{llllll}-7 & -8 & 2 & 115 & -74 & 13\end{array}$

$\begin{array}{rrrrrr}-6 & -8 & 2 & 233 & 245 & 7 \\ -5 & -8 & 2 & 213 & -220 & 7\end{array}$

$\begin{array}{rrrrr}-5 & -8 & 2 & 213 & -220 \\ -4 & -8 & 2 & 332 & 352\end{array}$

$\begin{array}{rrrrrr}-3 & -8 & 2 & 297 & -302 & 5\end{array}$

$\begin{array}{rrrrrr}-2 & -8 & 2 & 105 & 83 & 11 \\ -1 & -8 & 2 & 236 & -235 & \end{array}$

$\begin{array}{llllll}0 & -8 & 2 & 489 & 494 & 7\end{array}$

$\begin{array}{rrrrr}1 & -8 & 2 & 460 & -454 \\ 2 & -8 & 2 & 393 & 413\end{array}$

$\begin{array}{rrrrrr}2 & -8 & 2 & 393 & 413 & 6 \\ 3 & -8 & 2 & 392 & -403 & 7\end{array}$

$\begin{array}{llllll}4 & -8 & 2 & 49 & 66 & -24\end{array}$

$\begin{array}{llllll}5 & -8 & 2 & 155 & -167 & 12\end{array}$

$\begin{array}{llllll}6 & -8 & 2 & 134 & 130 & 13\end{array}$

$\begin{array}{rrrrrr}7 & -8 & 2 & 80 & -78 & -21 \\ 8 & -8 & 2 & 13 & 29 & -52\end{array}$

$\begin{array}{rrrrrr}-12 & -7 & 2 & 35 & 15 & -35 \\ -11 & -7 & 2 & 58 & -32 & -32\end{array}$

$\begin{array}{llllll}-10 & -7 & 2 & 60 & 68 & -29\end{array}$

$\begin{array}{llllll}-9 & -7 & 2 & 61 & -47 & -30\end{array}$

$\begin{array}{llllll}-8 & -7 & 2 & 48 & 15 & -35\end{array}$

$\begin{array}{llllll}-7 & -7 & 2 & 106 & -58 & 13 \\ -6 & -7 & 2 & 394 & 194 & 8\end{array}$

$\begin{array}{llllll}-5 & -7 & 2 & 126 & -97 & 10\end{array}$

$\begin{array}{llllll}-4 & -7 & 2 & 71 & 42 & -15\end{array}$

$\begin{array}{llllll}-3 & -7 & 2 & 23 & -29 & -36\end{array}$

$\begin{array}{rrrrrr}-2 & -7 & 2 & 1.16 & 123 & 9 \\ -1 & -7 & 2 & 1.86 & -1.88 & 7\end{array}$

$\begin{array}{llllll}0 & -7 & 2 & 146 & 139 & 9\end{array}$

$\begin{array}{rrrrrr}1 & -7 & 2 & 68 & -16 & -16 \\ 2 & -7 & 2 & 125 & 121 & 11\end{array}$ $\begin{array}{llllll}3-7 & 2 & 150 & -136 & 10\end{array}$

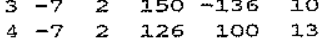

$\begin{array}{llllll}5 & -7 & 2 & 103 & -94 & 15\end{array}$

$\begin{array}{rrrrrr}6 & -7 & 2 & 59 & -67 & -32\end{array}$

$\begin{array}{llllll}7 & -7 & 2 & 96 & 67 & 17\end{array}$

$\begin{array}{llllll}8 & -7 & 2 & 63 & -47 & -30\end{array}$

$\begin{array}{rrrrrr}9 & -7 & 2 & 54 & -11 & -35 \\ -12 & -5 & 2 & 78 & 30 & -28\end{array}$

$\begin{array}{llllll}-11 & -6 & 2 & 76 & -33 & -19\end{array}$

$\begin{array}{llllll}-10 & -6 & 2 & 52 & 61 & -32\end{array}$

$\begin{array}{rrrrrr}-9 & -6 & 2 & 122 & -84 & 12 \\ -8 & -6 & 2 & 62 & 78 & -28\end{array}$

$\begin{array}{llllll}-7 & -6 & 2 & 135 & -127 & 10\end{array}$

$\begin{array}{lllll}-6 & -5 & 2 & 197 & 195\end{array}$

$\begin{array}{llllll}-5 & -6 & 2 & 320 & -304\end{array}$

$\begin{array}{lllll}-4 & -6 & 2 & 567 & 601\end{array}$

$\begin{array}{rrrrr}-3 & -6 & 2 & 657 & -652 \\ -2 & -6 & 2 & 261 & 265\end{array}$

$\begin{array}{lllll}-1 & -6 & 2 & 626 & -601\end{array}$

$\begin{array}{llll}-6 & 2 & 588 & 552\end{array}$

$\begin{array}{lllll}1 & -6 & 2 & 676 & -639\end{array}$

$\begin{array}{rrrrr}2 & -6 & 2 & 486 & 479 \\ 3 & -6 & 2 & 491 & -516\end{array}$

$\begin{array}{rrrrr}3 & -6 & 2 & 491 & -516 \\ 4 & -6 & 2 & 542 & 558\end{array}$

$\begin{array}{lllll}5 & -6 & 2 & 434 & -476\end{array}$

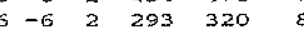

$\begin{array}{rrrrrr}7 & -6 & 2 & 139 & -151 & 12 \\ 8 & -6 & 2 & 13 & 76 & -50\end{array}$

$30-6 \quad 2 \quad 33 \quad 19-42$

$\begin{array}{llllll}-12 & -5 & 2 & 86 & -8 & -18\end{array}$

$\begin{array}{rrrrrr}-11 & -5 & 2 & 13 & 1 & -52 \\ -10 & -5 & 2 & 81 & 16 & -18\end{array}$

$\begin{array}{llllll}-9 & -5 & 2 & 97 & -97 & 15\end{array}$

$\begin{array}{llllll}-8 & -5 & 2 & 94 & 102 & 24\end{array}$

$\begin{array}{llllll}-7 & -5 & 2 & 123 & -60 & 12\end{array}$

$\begin{array}{rrrrrr}-6 & -5 & 2 & 106 & 75 & 11 \\ -5 & -5 & 2 & 72 & -97 & 14\end{array}$

$\begin{array}{rrrrr}-4 & -5 & 2 & 196 & -158\end{array}$

$\begin{array}{llllll}-3 & -5 & 2 & 523 & 519 & 6\end{array}$

$\begin{array}{llllll}-1 & -5 & 2 & 9 & 30 & -37\end{array}$

$\begin{array}{rrrrrr}-1 & -5 & 2 & 9 & 30 & -37 \\ 0 & -5 & 2 & 170 & -129\end{array}$

$\begin{array}{rrrrrr}1 & -5 & 2 & 89 & -17 & 11 \\ 2 & -5 & 2 & 138 & -145 & 8\end{array}$

$\begin{array}{lllll}3 & -5 & 2 & 251 & -238\end{array}$

$\begin{array}{llllll}4 & -5 & 2 & 174 & 168\end{array}$

$\begin{array}{llllll}5 & -5 & 2 & 89 & -74 & 13\end{array}$

$\begin{array}{rrrrrr}6 & -5 & 2 & 106 & 77 & 12\end{array}$

$\begin{array}{rrrrrr}7 & -5 & 2 & 89 & -22 & 17 \\ 8 & -5 & 2 & 62 & -37 & -28\end{array}$

$\begin{array}{llllll}9 & -5 & 2 & 70 & 18 & -30\end{array}$

$\begin{array}{rrrrrr}9 & -5 & 2 & 70 & 18 & -30 \\ 20 & -5 & 2 & 53 & -21 & -33\end{array}$

$\begin{array}{rrrrr}-13 & -4 & 2 & 51 & 6\end{array}$

$\begin{array}{llllll}-12 & -4 & 2 & 51 & 45 & -34 \\ -1 x & -4 & 2 & 61 & -4 & -27\end{array}$

$\begin{array}{llllll}-10 & -4 & 2 & 115 & 78 & 14\end{array}$

$\begin{array}{llllll}-9 & -4 & 2 & 89 & -80 & 16\end{array}$

$\begin{array}{llllll}-8 & -4 & 2 & 40 & -1.2 & -23\end{array}$

$\begin{array}{llllll}-7 & -4 & 2 & 169 & -181\end{array}$

$\begin{array}{rrrrr}-5 & -4 & 2 & 436 & 410 \\ -5 & 2 & 414 & -428\end{array}$

$\begin{array}{llllll}-4 & -4 & 2 & 494 & 501\end{array}$

$\begin{array}{lllll}-3 & -4 & 2 & 709 & -715\end{array}$

$\begin{array}{lllll}-2 & -4 & 2 & 822 & 819\end{array}$

$\begin{array}{rrrrr}-1 & -4 & 2 & 716 & -671 \\ 0 & -4 & 2 & 608 & 565\end{array}$

$\begin{array}{lllll}1 & -4 & 2 & 373 & -358\end{array}$

$\begin{array}{lllll}2 & -4 & 2 & 228 & 146\end{array}$

$\begin{array}{lllll}3 & -4 & 2 & 330 & -353\end{array}$

$\begin{array}{rrrrr}4 & -4 & 2 & 221 & 211 \\ 5 & -4 & 2 & 479 & -491\end{array}$

$\begin{array}{llllll}6 & -4 & 2 & 430 & 454\end{array}$

$7-42206-244$

$\begin{array}{rrrrrr}8 & -4 & 2 & 157 & 184 & 12 \\ 9 & -4 & 2 & 99 & -127 & 16\end{array}$

$\begin{array}{rrrrrr}9 & -4 & 2 & 99 & -127 & 16 \\ 10 & -4 & 2 & 48 & 46 & -36\end{array}$

$11-4 \quad 2 \quad 57 \quad-9 \quad-34$

$\begin{array}{lllll}-12 & -3 & 2 & 13 & -52\end{array}$

$\begin{array}{llllll}-11 & -3 & 2 & 53 & -6 & -28 \\ -10 & -3 & 2 & 42 & 15 & -36\end{array}$

$\begin{array}{rrrrrr}-9 & -3 & 2 & 87 & 54 & -36 \\ -8 & -3 & 2 & & -22 & -36\end{array}$

$\begin{array}{rrrrrr}-8 & -3 & 2 & 9 & -22 & -36 \\ -7 & -3 & 2 & 338 & 77 & 9\end{array}$

$\begin{array}{rrrrrr}-7 & -3 & 2 & 138 & 77 & 9 \\ -6 & -3 & 2 & 93 & -81 & 12\end{array}$

$\begin{array}{llllll}-5 & -3 & 2 & 93 & -81 & 12 \\ -5 & 2 & 35 & -13 & -27\end{array}$

$\begin{array}{lllll}-4 & -3 & 2 & 155 & 137\end{array}$

$\begin{array}{lllll}-3 & -3 & 2 & 233 & 212\end{array}$

$\begin{array}{lllll}-2 & -3 & 2 & 671 & -653\end{array}$

$\begin{array}{rrrrrr}-1 & -3 & 2 & 529 & 491 & 4 \\ 0 & -3 & 2 & 44 & 38 & -22\end{array}$ $\mathrm{k} 1$ 10Fo 10FC 10s

h $\mathrm{k} 1$ 1OFO IOFO $10 \mathrm{~S}$

$\begin{array}{llllllllllll}1 & -3 & 2 & 351 & 316 & 5 & -8 & 1 & 2 & 122 & 139 & 12\end{array}$

$\begin{array}{rrrrrr}2 & -3 & 2 & 205 & -207 & 5 \\ 3 & -3 & 2 & 64 & 45 & 12\end{array}$

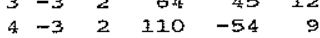

$\begin{array}{llllll}5 & -3 & 2 & 42 & -19 & -25\end{array}$

$6-3 \quad 2 \quad 151-151 \quad 8$

$\begin{array}{rrrrrr}7 & -3 & 2 & 110 & 32 & 12 \\ 8 & -3 & 2 & 91 & -73 & 16\end{array}$

$\begin{array}{llllll}9 & -3 & 2 & 103 & -30 & 14\end{array}$

$\begin{array}{llllll}10 & -3 & 2 & 91 & -34 & 17\end{array}$

$\begin{array}{rrrrrr}11 & -3 & 2 & 69 & 31 & -29\end{array}$

$\begin{array}{rrrrrr}-12 & -2 & 2 & 80 & 44 & -19 \\ -11 & -2 & 2 & 72 & -98 & -20\end{array}$

$\begin{array}{llllll}-10 & -2 & 2 & 88 & 66 & 16\end{array}$

$\begin{array}{lllllll}-9 & -2 & 2 & 164 & -184 & 10\end{array}$

$\begin{array}{llllll}-8 & -2 & 2 & 146 & 133 & 10\end{array}$

$\begin{array}{llllll}-7 & -2 & 2 & 125 & -109 & 10\end{array}$

$\begin{array}{lllll}-6 & -2 & 2 & 422 & 451\end{array}$

$\begin{array}{lllll}-5 & -2 & 2 & 182 & -190\end{array}$

$\begin{array}{lllll}-3 & -2 & 2 & 202 & -202\end{array}$

$\begin{array}{lllll}-2 & -2 & 2 & 465 & 492\end{array}$

$\begin{array}{llll}-1 & -2 & 2 & 1622-3.607\end{array}$

$\begin{array}{llll}-2 & 2 & 286 & 281\end{array}$

]. $\begin{array}{lllll}-2 & 2 & 101 & -75 & 8\end{array}$

$\begin{array}{rrrrr}2 & -2 & 2 & 75 & 77 \\ 3 & -2 & 2 & 682 & -657\end{array}$

$\begin{array}{rrrrr}3 & -2 & 2 & 682 & -657 \\ 4 & -2 & 2 & 531 & 546\end{array}$

$\begin{array}{lllllll}5 & -2 & 2 & 348 & -354 & 5\end{array}$

$\begin{array}{llllll}6 & -2 & 2 & 216 & 222 & 7\end{array}$

$\begin{array}{rrrrrr}7 & -2 & 2 & 126 & -113 & 11 \\ 8 & -2 & 2 & 137 & 129 & 10\end{array}$

$\begin{array}{rrrrrr}8 & -2 & 2 & 137 & 129 & 10 \\ 9 & -2 & 2 & 156 & -180 & 11\end{array}$

$\begin{array}{llllll}30 & -2 & 2 & 65 & 36 & -29\end{array}$

$\begin{array}{llllll}11 & -2 & 2 & 93 & -82 & 38\end{array}$

$\begin{array}{llllll}12 & -2 & 2 & 97 & 12 & -21\end{array}$

$\begin{array}{rrrrrr}-12 & -1 & 2 & 32 & 2 & -46 \\ -11 & -1 & 2 & 66 & 36 & -28\end{array}$

$\begin{array}{llllll}-11 & -1 & 2 & 66 & 36 & -28 \\ -10 & -1 & 2 & 45 & 22 & -32\end{array}$

$\begin{array}{llllll}-9 & -1 & 2 & 110 & -98 & 12\end{array}$

$\begin{array}{llllll}-8 & -1 & 2 & 108 & -72 & 12\end{array}$

$\begin{array}{rrrrrr}-7 & -1 & 2 & 76 & -28 & 15 \\ -6 & -1 & 2 & 115 & 88 & 10\end{array}$

$\begin{array}{rrrrrr}-5 & -1 & 2 & 130 & 151 & 8\end{array}$

$\begin{array}{llllll}-4 & -1 & 2 & 102 & -7 & -9\end{array}$

$\begin{array}{rrrrrr}-3 & -1 & 2 & 492 & 499 & 5 \\ -2 & -1 & 2 & 71 & -66 & 12\end{array}$

$\begin{array}{lllll}-1 & -1 & 2 & 319 & 355\end{array}$

$\begin{array}{lllll}0 & -x & 2 & 210 & -176 \\ 1 & -1 & 2 & 110 & 104\end{array}$

$2-1 \quad 2$ 1.24-1061

$\begin{array}{lllll}3 & -1 & 2 & 235 & 237\end{array}$

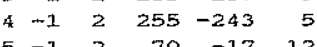

$\begin{array}{rrrrrr}5 & -1 & 2 & 70 & -17 & 12 \\ 6 & -1 & 2 & 165 & 147 & 7\end{array}$

$\begin{array}{llllll}7 & -1 & 2 & 134 & 78 & 9\end{array}$

$\begin{array}{llllll}8 & -1 & 2 & 101 & -93 & 12\end{array}$

$\begin{array}{rrrrrr}9 & -1 & 2 & 126 & 121 & 12 \\ 10 & -1 & 2 & 92 & 6 & 15\end{array}$

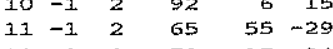

$\begin{array}{llllll}12 & -1 & 2 & 59 & 15 & -34\end{array}$

$\begin{array}{llllll}-12 & 0 & 2 & 58 & 42 & -23\end{array}$

$\begin{array}{lllllll}-12 & 0 & 2 & 43 & 9 & -25\end{array}$

$\begin{array}{rrrrrr}-10 & 0 & 2 & 127 & 111 & 10 \\ -9 & 0 & 2 & 65 & -34 & -18\end{array}$

$\begin{array}{rrrrrr}-9 & 0 & 2 & 65 & -34 & -18 \\ -8 & 0 & 2 & 66 & 67 & -19\end{array}$

$\begin{array}{lllll}-7 & 0 & 2 & 226 & -223\end{array}$

$\begin{array}{lllll}-6 & 0 & 2 & 432 & 455\end{array}$

$\begin{array}{lllll}-5 & 0 & 2 & 579 & -601\end{array}$

$\begin{array}{lllll}-4 & 0 & 2 & 310 & 301\end{array}$

$\begin{array}{rrrrr}-3 & 0 & 2 & 421 & -416 \\ -2 & 0 & 2 & 336 & -297\end{array}$

$-1021038-1048$

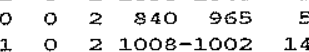

$2 \quad 0 \quad 2 \quad 451-415$

$3002606-621$

$\begin{array}{rrrrr}4 & 0 & 2 & 79 & 48 \\ 5 & 0 & 2 & 487 & -472\end{array}$

$6 \quad 0 \quad 2 \quad 296 \quad 283$

$\begin{array}{llllrr}7 & 0 & 2 & 222 & -218 & 5 \\ 8 & 0 & 2 & 136 & 105 & 10\end{array}$

$902265-180 \quad 22$

$10 \quad 0 \quad 2 \quad 84 \quad 64 \quad 15$

$\begin{array}{rrrrrr}21 & 0 & 2 & 41 & 21 & -26 \\ 12 & 0 & 2 & 91 . & 87 & 13\end{array}$

$\begin{array}{rrrrrr}-12 & 1 & 2 & 56 & 33 & -23\end{array}$

$\begin{array}{rrrrrr}-11 & 1 & 2 & 25 & 7 & -42 \\ -10 & 1 & 2 & 47 & -12 & -34\end{array}$

$\begin{array}{rrrrrr}-10 & 1 & 2 & 47 & -12 & -34 \\ -9 & 1 & 2 & 80 & 5 & -17\end{array}$ $\begin{array}{lllll}-7 & 2 & 105 & -20 & 11\end{array}$

$\begin{array}{rrrrrr}-6 & 1 & 2 & 204 & 206 & 7 \\ -5 & 3 & 2 & 66 & -14 & -14\end{array}$

$\begin{array}{llllll}-4 & 1 & 2 & 124 & -39 & -8\end{array}$

$\begin{array}{rrrrrr}-3 & 1 & 2 & 222 & -228 & 5 \\ -2 & 1 & 2 & 239 & 172 & -5\end{array}$

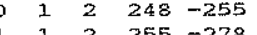

$\begin{array}{lllll}1 & 1 & 2 & 293 & 303\end{array}$

$312 \quad 1057-1069$

$12350 \quad 367$

$\begin{array}{lllll}6 & 1 & 2 & 149 & -108\end{array}$

$\begin{array}{lllll}7 & 1 & 2 & 138 & 97\end{array}$

$\begin{array}{rrrrrr}8 & 1 & 2 & 119 & -111 & 10 \\ 9 & 1 & 2 & 91 & -25 & 14\end{array}$

$\begin{array}{llllll}10 & 1 & 2 & 59 & -40 & -28\end{array}$

$\begin{array}{llllll}11 & 1 & 2 & 34 & 41 & -33\end{array}$

$\begin{array}{llllll}12 & 1 & 2 & 59 & 10 & -32\end{array}$

$\begin{array}{rrrrrr}13 & 3 & 2 & 50 & 11 & -37 \\ -21 & 2 & 2 & 76 & -42 & -20\end{array}$

$\begin{array}{llllll}-10 & 2 & 2 & 76 & 53 & -20\end{array}$

$\begin{array}{rrrrrr}-9 & 2 & 2 & 122 & -147 & 14 \\ -8 & 2 & 2 & 48 & 15 & -32\end{array}$

$\begin{array}{llllll}-7 & 2 & 2 & 105 & -65 & 12\end{array}$

$\begin{array}{llllr}-6 & 2 & 2 & 398 & 427 \\ -5 & 2 & 2 & 519 & -515\end{array}$

$\begin{array}{lllll}-4 & 2 & 2 & 413 & 412\end{array}$

$\begin{array}{lllll}-3 & 2 & 2 & 813 & -822\end{array}$

$\begin{array}{rrrrr}-2 & 2 & 2 & 477 & 477 \\ -1 & 2 & 2 & 1530-1381\end{array}$

- 221.202984

$\begin{array}{lllll}1 & 2 & 2 & 104 & -128 \\ 2 & 2 & 2 & 292 & -260\end{array}$

322 1073-1102

$\begin{array}{rrrrr}4 & 2 & 2 & 1025 & 1035 \\ 5 & 2 & 2 & 852 & -875\end{array}$

$622804 \quad 835$

$22168-163$

$\begin{array}{llllll}8 & 2 & 2 & 94 & 112 & 13\end{array}$

$\begin{array}{rrrrrr}9 & 2 & 2 & 131 & -129 & 11 \\ 10 & 2 & 2 & 41 & 29 & -35\end{array}$

$1122 \quad 12 \quad-61-48$

$\begin{array}{llllll}12 & 2 & 2 & 66 & 41 & -29\end{array}$

$\begin{array}{llllll}13 & 2 & 2 & 42 & -25 & -41\end{array}$

$\begin{array}{rrrrrr}-11 & 3 & 2 & 38 & 33 & -41 \\ -10 & 3 & 2 & 25 & -49 & -48\end{array}$

$\begin{array}{llllll}-9 & 3 & 2 & 62 & -26 & -26\end{array}$

$\begin{array}{llllll}-8 & 3 & 2 & 59 & -70 & -29\end{array}$

$\begin{array}{llllll}-7 & 3 & 2 & 64 & -33 & -18\end{array}$

$\begin{array}{rrrrr}-6 & 3 & 2 & 178 & 190 \\ -5 & 3 & 2 & 187 & -184\end{array}$

$\begin{array}{lllll}-4 & 3 & 2 & 140 & -89\end{array}$

$\begin{array}{lllll}-3 & 3 & 2 & 128 & -99\end{array}$

$\begin{array}{lllll}-2 & 3 & 2 & 196 & 172\end{array}$

$\begin{array}{lllll}-1 & 3 & 2 & 176 & -132\end{array}$

$\begin{array}{rrrrr}0 & 3 & 2 & 467 & 403 \\ 1 & 3 & 2 & 575 & -503\end{array}$

$\begin{array}{lllll}2 & 3 & 2 & 315 & -296\end{array}$

$\begin{array}{rrrrrr}3 & 3 & 2 & 94 & -76 & 10 \\ 4 & 3 & 2 & 233 & 227 & 5\end{array}$

$\begin{array}{lllll}5 & 3 & 2 & 414 & -400 \\ 6 & 3 & 2 & 503 & 538\end{array}$

$\begin{array}{rrrrr}6 & 3 & 2 & 501 & 538 \\ 7 & 3 & 2 & 277 & -321\end{array}$

$\begin{array}{llllrl}8 & 3 & 2 & 103 & 75 & 12\end{array}$

$\begin{array}{llllll}11 & 3 & 2 & 23 & 72 & -45\end{array}$

$\begin{array}{llllll}12 & 3 & 2 & 58 & -18 & -34\end{array}$

$\begin{array}{rrrrrr}13 & 3 & 2 & 59 & -8 & -32 \\ -10 & 4 & 2 & 76 & 13 & -26\end{array}$

$\begin{array}{llllll}-9 & 2 & 72 & -60 & -19\end{array}$

$\begin{array}{rrrrrr}-8 & 4 & 2 & 51 & 36 & -32 \\ -7 & 4 & 2 & 156 & -154 & 11\end{array}$

$\begin{array}{rrrrr}-7 & 4 & 2 & 156 & -154 \\ -6 & 4 & 2 & 241 & 239\end{array}$

$\begin{array}{lllll}-5 & 4 & 2 & 182 & -166\end{array}$

$\begin{array}{lllll}-4 & 4 & 2 & 250 & 245\end{array}$

$\begin{array}{lllll}-2 & 4 & 2 & 277 & 263\end{array}$

$\begin{array}{lllll}-1 & 4 & 2 & 546 & -474\end{array}$

$\begin{array}{llll}2 & 457 & -444\end{array}$

$34 \quad 2 \quad 617-616$

$\begin{array}{rrrrr}4 & 4 & 2 & 271 & 267 \\ 5 & 4 & 2 & 71 & 45\end{array}$

$\begin{array}{rrrrr}6 & 4 & 2 & 315 & 314 \\ 7 & 4 & 2 & 388 & -407\end{array}$ $\begin{array}{lllll}-1 & 1 & 2 & 238 & 255\end{array}$

$\begin{array}{rrrrrr}9 & 3 & 2 & 231 & 108 & 12 \\ 10 & 3 & 2 & 67 & -19 & -21\end{array}$

421089971

h $\mathrm{ke} 1$ 1OFO IOFC $10 \mathrm{~S}$

8423633876

$\begin{array}{rrrrrr}9 & 4 & 2 & 318 & -359 & 7 \\ 10 & 4 & 2 & 49 & 117 & -30\end{array}$

$11 \quad 4 \quad 2 \quad 62 \quad 36-30$

$\begin{array}{llllll}12 & 4 & 2 & 63 & 34 & -28\end{array}$

$\begin{array}{rrrrrr}-10 & 5 & 2 & 86 & 27 & -20 \\ -9 & 5 & 2 & 48 & 27 & -36\end{array}$

$\begin{array}{llllll}-8 & 5 & 2 & 66 & -42 & -28\end{array}$ 
h $\mathrm{k}$ l 10FO $10 \mathrm{FC} 10 \mathrm{~s}$

$\begin{array}{llllll}7 & 8 & 2 & 112 & -93 & 13\end{array}$

$\begin{array}{rrrrrr}8 & 8 & 2 & 112 & 135 & 14 \\ 9 & 8 & 2 & 94 & -74 & 16\end{array}$

$\begin{array}{llllll}10 & 8 & 2 & 111 & 95 & 14\end{array}$

12822 65 $23-31$

$\begin{array}{llllll}13 & 8 & 2 & 50 & 16 & -39 \\ -7 & 9 & 2 & 63 & 32 & -32\end{array}$

$\begin{array}{llllll}-6 & 9 & 2 & 75 & -7 & -20\end{array}$

$\begin{array}{rrrrrr}-5 & 9 & 2 & 98 & -7 & 15 \\ -4 & 9 & 2 & 62 & -48 & -29\end{array}$

$\begin{array}{rrrrrr}-3 & 9 & 2 & 42 & 30 & -35 \\ -2 & 9 & 2 & 11 & -28 & -44\end{array}$

$\begin{array}{llllll}-1 & 9 & 2 & 33 & -34 & -37\end{array}$

$\begin{array}{rrrrrr}0 & 9 & 2 & 196 & -210 & 8 \\ 1 & 9 & 2 & 76 & -77 & -16\end{array}$

$\begin{array}{llllll}2 & 9 & 2 & 123 & 113 & 10 \\ 3 & 9 & 2 & 105 & -60 & 10\end{array}$

492 210 2028

$\begin{array}{rrrrrr}5 & 9 & 2 & 195 & -185 & 8 \\ 6 & 9 & 2 & 153 & 143 & 11\end{array}$

$\begin{array}{rrrrrr}7 & 9 & 2 & 125 & -108 & 14 \\ 8 & 9 & 2 & 93 & 44 & 16\end{array}$

$\begin{array}{llllll}9 & 9 & 51 & -16 & -31\end{array}$

$\begin{array}{llllll}10 & 9 & 2 & 58 & 18 & -31 \\ 11 & 9 & 2 & 35 & -3 & -39\end{array}$

$\begin{array}{rrrrrr}12 & 9 & 2 & 67 & 24 & -31 \\ -6 & 10 & 2 & 105 & 144 & 19\end{array}$

$\begin{array}{llllll}-5 & 10 & 2 & 102 & -129 & 18\end{array}$

$\begin{array}{llllll}-4 & 10 & 2 & 142 & 149 & 14\end{array}$

$\begin{array}{rrrrrr}-3 & 10 & 2 & 284 & -309 & 9 \\ -2 & 10 & 2 & 268 & 289 & 9\end{array}$

$\begin{array}{rrrrrr}-1 & 10 & 2 & 190 & -188 & 10 \\ 0 & 10 & 2 & 154 & 157 & 11\end{array}$

$110 \quad 2 \quad \begin{array}{llll}159 & -161 & 11\end{array}$

$210220 \quad 44-40$

$\begin{array}{rrrrrr}3 & 10 & 2 & 201 & -178 & 8 \\ 4 & 10 & 2 & 259 & 262 & 8\end{array}$

$510 \quad 2 \quad 186-191 \quad 10$

$\begin{array}{llllll}6 & 10 & 2 & 238 & 244 & 8\end{array}$

$\begin{array}{llllll}7 & 10 & 2 & 192 & -202 & 10\end{array}$

81029110618

$\begin{array}{rrrrrr}9 & 10 & 2 & 69 & -86 & -23 \\ 10 & 10 & 2 & 205 & 34 & 14\end{array}$

11 $102258 \quad-34 \quad-32$

$\begin{array}{llllll}12 & 10 & 2 & 74 & 19 & -30\end{array}$

$\begin{array}{llllll}-5 & 11 & 2 & 43 & -37 & -41\end{array}$

$\begin{array}{llllll}-4 & 11 & 2 & 49 & 44 & -35 \\ -3 & 11 & 2 & 53 & -7 & -34\end{array}$

$\begin{array}{llllll}-3 & 11 & 2 & 53 & -7 & -34 \\ -2 & 11 & 2 & 24 & -7 & -46\end{array}$

$\begin{array}{llllll}-1 & 11 & 2 & 125 & -135 & 14\end{array}$

$\begin{array}{rrrrrr}0 & 11 & 2 & 127 & 140 & 14 \\ 1 & 11 & 2 & 60 & -48 & -28\end{array}$

$\begin{array}{rrrrrr}3 & 11 & 2 & 74 & -38 & -19 \\ 1 & 11 & 2 & 55 & 39 & -21\end{array}$

$\begin{array}{llllll}5 & 11 & 2 & 59 & -57 & -31\end{array}$

$6112159 \quad 148 \quad 13$

$\begin{array}{rrrrrr}7 & 11 & 2 & 156 & -135 & 12 \\ 8 & 11 & 2 & 126 & 110 & 14\end{array}$

$911 \quad 2 \quad 43-107-41$

$\begin{array}{lllll}1011 & 2 & 51 & 64 & -35\end{array}$

$\begin{array}{llllll}11 & 11 & 2 & 53 & -31 & -36\end{array}$

$\begin{array}{llllll}-3 & 12 & 2 & 105 & -162 & 19\end{array}$

$\begin{array}{llllll}-2 & 12 & 2 & 165 & 176 & 12\end{array}$

$\begin{array}{rrrrrr}-1 & 12 & 2 & 137 & -133 & 14 \\ 0 & 12 & 2 & 90 & 97 & -19\end{array}$

$\begin{array}{llllll}1 & 12 & 2 & 204 & -196 & 10\end{array}$

$2122221219 \quad 10$

$\begin{array}{llllll}3 & 12 & 2 & 129 & -124 & 14\end{array}$

$\begin{array}{rrrrrr}4 & 12 & 2 & 43 & 80 & -32 \\ 5 & 12 & 2 & 85 & -85 & -18\end{array}$

$\begin{array}{llllll}6 & 12 & 2 & 154 & 174 & 13\end{array}$

$\begin{array}{llllll}7 & 12 & 2 & 149 & -152 & 13\end{array}$

$\begin{array}{llllll}8 & 12 & 2 & 99 & 127 & 18\end{array}$

$\begin{array}{rrrrrr}9 & 12 & 2 & 83 & -77 & -21 \\ 10 & 12 & 2 & 62 & 30 & -33\end{array}$

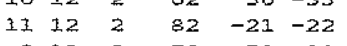

$\begin{array}{llllll}-2 & 13 & 2 & 72 & 59 & -32\end{array}$

$\begin{array}{llllll}-1 & 13 & 2 & 50 & -85 & -37\end{array}$

$\begin{array}{llllll}-13 & 2 & 32 & 91 & -45\end{array}$

$\begin{array}{llllll}1 & 1.3 & 2 & 80 & -87 & -22 \\ 2 & 13 & 2 & 59 & -17 & -33\end{array}$

$\begin{array}{llllll}313 & 2 & 56 & -26 & -32\end{array}$

$413257 \quad 61-30$

$\begin{array}{llllll}5 & 13 & 2 & 76 & -10 & -20 \\ 6 & 13 & 2 & 78 & -28 & -29\end{array}$

$\begin{array}{rrrrrr}6 & 13 & 2 & 78 & -28 & -29 \\ 7 & 13 & 2 & 92 & 3 & -19\end{array}$

$823210840 \quad 16$

$\begin{array}{llllll}9 & 13 & 2 & 82 & -65 & -25\end{array}$

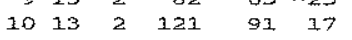

$\begin{array}{rrrrrr}2 & 14 & 2 & 33 & 121 & -51\end{array}$

$3142141-159 \quad 15$

$4142123 \quad 118 \quad 16$

$51 \div 246 \quad-78-35$

$\begin{array}{llllll}6 & 14 & 2 & 66 & 90 & -33 \\ 8 & 14 & 2 & 52 & -2 & -39\end{array}$

$\begin{array}{lllll}-4-14 & 3 & 121 & 97 & 15\end{array}$

$\begin{array}{lllll}-3-14 & 3 & 50 & -74 & -40\end{array}$

$\begin{array}{lllll}-7-13 & 3 & 73 & -26 & -31 \\ -6-13 & 3 & 66 & 78 & -25\end{array}$

$\begin{array}{rrrrr}-6-13 & 3 & 66 & 78 & -25 \\ -5-13 & 3 & 114 & -110 & 15\end{array}$

$\begin{array}{lllll}-4-13 & 3 & 123 & 123 & 14\end{array}$

$\begin{array}{lllll}-3-13 & 3 & 144 & -157 & 14\end{array}$

$\begin{array}{rrrrr}-2-13 & 3 & 267 & 161 & 13 \\ -1-13 & 3 & 110 & -118 & 17\end{array}$

$\begin{array}{rrrrr}-1-13 & 3 & 110 & -118 & 17 \\ 0-13 & 3 & 92 & 96 & -20\end{array}$

$\begin{array}{lllll}1-23 & 3 & 34 & -65 & -48\end{array}$

$\begin{array}{lllll}-9-12 & 3 & 90 & -78 & -21\end{array}$

$\begin{array}{lllll}-8-12 & 3 & 127 & 105 & 15\end{array}$

$\begin{array}{lllll}-7-12 & 3 & 139 & -126 & 14\end{array}$

$\begin{array}{lllrl}-6-12 & 3 & 53 & 90 & -29 \\ -5-12 & 3 & 39 & -64 & -34\end{array}$

$\begin{array}{lllll}-4-22 & 3 & 73 & 101 & -20\end{array}$

$\begin{array}{lllll}-3-12 & 3 & 121 & -131 & 15\end{array}$

$\begin{array}{lllll}-2-12 & 3 & 41 & 51 & -37\end{array}$

$\begin{array}{rrrrr}-1-12 & 3 & 30 & -31 & -45 \\ 0-12 & 3 & 41 & 50 & -32\end{array}$

$\begin{array}{lllll}1-12 & 3 & 97 & -45 & -24\end{array}$

$\begin{array}{lllll}2-12 & 3 & 66 & 75 & -31\end{array}$

$\begin{array}{lllll}3-12 & 3 & 64 & -25 & -31\end{array}$

$\begin{array}{lllll}-10-11 & 3 & 46 & -12 & -38\end{array}$

$\begin{array}{lllll}-9-11 & 3 & 68 & 24 & -31 \\ -8-11 & 3 & 67 & 24 & -30\end{array}$

$\begin{array}{lllll}-7-11 & 3 & 98 & -76 & 17\end{array}$

$\begin{array}{lllll}-6-11 & 3 & 123 & 230 & 13\end{array}$

$-5-11 \quad 3 \quad 63-205-28$

$\begin{array}{rrrrr}-4-11 & 3 & 90 & 125 & 15 \\ -3-11 & 3 & 191 & -205 & 10\end{array}$

$-2-11 \quad 3 \quad 248 \quad 246 \quad 9$

$\begin{array}{lllll}-1-11 & 3 & 253 & -256 & 9\end{array}$

$0-11 \quad 3 \quad 224 \quad 230 \quad 10$

$\begin{array}{rrrrr}1-1.1 & 3 & 160 & -154 & 12 \\ 2-11 & 3 & 124 & 114 & 15\end{array}$

$3+11 \quad 3 \quad 105-109 \quad 18$

$\begin{array}{lllll}4-11 & 3 & 50 & 98 & -38\end{array}$

$\begin{array}{lllll}5-1 x & 3 & 109 & -72 & -26\end{array}$

$\begin{array}{rrrrr}-11-10 & 3 & 72 & -34 & -31 \\ -10-10 & 3 & 57 & 29 & -27\end{array}$

$\begin{array}{rrrrr}10-10 & 3 & 57 & 29 & -27 \\ -9-10 & 3 & 13 & -8 & -53\end{array}$

$\begin{array}{lllll}-8+10 & 3 & 55 & -30 & -31\end{array}$

$\begin{array}{lllll}-7-10 & 3 & 81 & -12 & -24 \\ -6-10 & 3 & 71 & 19 & -19\end{array}$

$\begin{array}{lllll}-5-10 & 3 & 62 & -49 & -20 \\ -4-10 & 3 & 77 & 120 & -17\end{array}$

$\begin{array}{lllll}-3-10 & 3 & 28 & -49 & -40\end{array}$

$\begin{array}{lllll}-2-10 & 3 & 50 & -78 & -31\end{array}$

$\begin{array}{lllll}-1-10 & 3 & 60 & 13 & -27\end{array}$

$\begin{array}{lllll}-10 & 3 & 82 & 52 & -24\end{array}$

$\begin{array}{rrrrr}1-10 & 3 & 57 & -55 & -23 \\ 2-10 & 3 & 47 & 44 & -33\end{array}$

$\begin{array}{lllll}3-10 & 3 & 49 & -50 & -28\end{array}$

$\begin{array}{lllll}4-10 & 3 & 120 & -26 & 21\end{array}$

$\begin{array}{lllll}5-10 & 3 & 37 & -28 & -47\end{array}$

$\begin{array}{llllll}-11 & -9 & 3 & 62 & 10 & -31 \\ -10 & -9 & 3 & 67 & 52 & -29\end{array}$

$\begin{array}{llllll}-9 & -9 & 3 & 90 & -87 & 17\end{array}$

$\begin{array}{llllll}-8 & -9 & 3 & 114 & 102 & 13\end{array}$

$\begin{array}{llllll}-7 & -9 & 3 & 46 & -48 & -33\end{array}$

$\begin{array}{rrrrrr}-6 & -9 & 3 & 136 & 116 & 12 \\ -5 & -9 & 3 & 143 & -163 & 11\end{array}$

$\begin{array}{llllll}-4 & -9 & 3 & 127 & 149 & 12\end{array}$

$\begin{array}{lllllllllll}-3 & -9 & 3 & 170 & -187 & 8\end{array}$

$\begin{array}{rrrrrr}-2 & -9 & 3 & 198 & 212 & 8 \\ -1 & -9 & 3 & 177 & -181 & 8\end{array}$

$\begin{array}{rrrrrr}-1 & -9 & 3 & 177 & -181 & 8 \\ 0 & -9 & 3 & 212 & 217 & 9\end{array}$

$\begin{array}{llllll}1 & -9 & 3 & 145 & -157 & 1.2\end{array}$

$\begin{array}{llllll}2 & -9 & 3 & 220 & 242 & 10\end{array}$

$\begin{array}{llllll}3 & -9 & 3 & 157 & -180 & 11\end{array}$

$\begin{array}{llllll}4 & -9 & 3 & 139 & 107 & 11\end{array}$

$\begin{array}{llllll}5 & -9 & 3 & 155 & -160 & 13\end{array}$

$\begin{array}{rrrrrr}7 & -9 & 3 & 106 & 150 & 17 \\ -9 & 3 & 54 & -120 & -38\end{array}$

$\begin{array}{llllll}-12 & -8 & 3 & 27 & -4 & -50\end{array}$

$\begin{array}{rrrrrr}-11 & -8 & 3 & 37 & 5 & -41\end{array}$

$\begin{array}{rrrrrr}-10 & -8 & 3 & 51 & 18 & -31 \\ -9 & -8 & 3 & 91 & -21 & -21\end{array}$

$\begin{array}{llllll}-8 & -8 & 3 & 56 & 4 & -28\end{array}$

$\begin{array}{llllll}-7 & -8 & 3 & 81 & -40 & -17 \\ -6 & -8 & 3 & 78 & 54 & -17\end{array}$

$h \mathrm{k} I$ 1OFO IOF̈C IOS

h $\mathrm{k}$ 1OFO IOFC $10 \mathrm{~s}$

h $k$ l IOFO IOFG IOS

$\begin{array}{rrrrrr}-5 & -8 & 3 & 154 & 129 & 9 \\ -4 & -8 & 3 & 69 & -40 & -16 \\ -3 & -8 & 3 & 118 & -146 & 10 \\ -2 & -8 & 3 & 35 & -59 & -30 \\ -1 & -8 & 3 & 60 & 22 & -17 \\ 0 & -8 & 3 & 126 & -122 & 11 \\ 1 & -8 & 3 & 24 & 24 & -32 \\ 2 & -8 & 3 & 36 & 20 & -34 \\ 3 & -8 & 3 & 121 & 110 & 14 \\ 4 & -8 & 3 & 35 & -47 & -36 \\ 5 & -8 & 3 & 13 & 55 & -53 \\ 6 & -8 & 3 & 95 & -49 & 16 \\ 7 & -8 & 3 & 45 & 54 & -36 \\ 8 & -8 & 3 & 47 & -10 & -37 \\ -12 & -7 & 3 & 46 & 46 & -37\end{array}$

$\begin{array}{llllll}-11 & -7 & 3 & 13 & -56 & -53\end{array}$

$\begin{array}{rrrrrr}-10 & -7 & 3 & 51 & 15 & -32 \\ -9 & -7 & 3 & 81 & -73 & -17\end{array}$

$\begin{array}{llllll}-8 & -7 & 3 & 82 & 82 & -19\end{array}$

$\begin{array}{lllllll}-7 & -7 & 3 & 193 & -194\end{array}$

$\begin{array}{lllll}-6 & -7 & 3 & 172 & 165\end{array}$

$\begin{array}{rrrrr}-5 & -7 & 3 & 265 & -278 \\ -4 & -7 & 3 & 456 & 500\end{array}$

$\begin{array}{lllll}-3 & -7 & 3 & 279 & -283\end{array}$

$\begin{array}{lllll}-2 & -7 & 3 & 230 & 233\end{array}$

$\begin{array}{llllll}-1 & -7 & 3 & 347 & -338\end{array}$

$\begin{array}{rrrrr}0 & -7 & 3 & 464 & 454 \\ 1 & -7 & 3 & 427 & -444\end{array}$

$\begin{array}{lllll}2 & -7 & 3 & 209 & 203\end{array}$

$\begin{array}{lllll}3 & -7 & 3 & 289 & -315\end{array}$

$\begin{array}{lllll}4 & -7 & 3 & 248 & 266\end{array}$

$\begin{array}{lllll}5 & -7 & 3 & 217 & -216\end{array}$

$\begin{array}{rrrrrr}6 & -7 & 3 & 232 & 257 & 9 \\ 7 & -7 & 3 & 138 & -118 & 14\end{array}$

$\begin{array}{llllll}8 & -7 & 3 & 89 & 67 & 18\end{array}$

$\begin{array}{llllll}9 & -7 & 3 & 66 & -24 & -29\end{array}$

$\begin{array}{llllll}-12 & -6 & 3 & 78 & 22 & -22\end{array}$

$\begin{array}{rrrrrr}-11 & -6 & 3 & 53 & 1 & -32 \\ -10 & -6 & 3 & 82 & 55 & -18\end{array}$

$\begin{array}{llllll}-9 & -5 & 3 & 50 & -56 & -33\end{array}$

$\begin{array}{llllll}-8 & -6 & 3 & 83 & -6 & 15\end{array}$

$\begin{array}{llllll}-7 & -6 & 3 & 102 & -81 & 22\end{array}$

$\begin{array}{rrrrrr}-6 & -6 & 3 & 118 & 100 & 11 \\ -5 & -6 & 3 & 340 & -358 & 6\end{array}$

$\begin{array}{llllll}-4 & -6 & 3 & 91 & -19 & 12\end{array}$

$\begin{array}{llllll}-3 & -6 & 3 & 292 & 162 & 6\end{array}$

$\begin{array}{rrrrrr}-2 & -6 & 3 & 63 & -23 & 12 \\ -1 & -6 & 3 & 79 & 61 & 10\end{array}$

$\begin{array}{llllll}0 & -6 & 3 & 23 & -17 & -27\end{array}$

$\begin{array}{rrrrrr}1 & -6 & 3 & 32 & 41 & -33 \\ 2 & -6 & 3 & 154 & 121 & 8\end{array}$

$\begin{array}{rrrrrr}3 & -6 & 3 & 72 & 26 & -15\end{array}$

$\begin{array}{llllll}4 & -6 & 3 & 101 & -56 & 13\end{array}$

$\begin{array}{rrrrrr}5 & -6 & 3 & 162 & -122 & 10 \\ 6 & -6 & 3 & 86 & 102 & -20\end{array}$

$\begin{array}{llllll}7 & -6 & 3 & 68 & -1 & -22\end{array}$

$\begin{array}{lllllll}8 & -6 & 3 & 42 & -43 & -34\end{array}$

$\begin{array}{rrrrrr}9 & -6 & 3 & 41 & 52 & -38 \\ 10 & -6 & 3 & 96 & -6 & 17\end{array}$

$\begin{array}{rrrrrr}-12 & -5 & 3 & 87 & 14 & -18 \\ -11 & -5 & 3 & 63 & -26 & -27\end{array}$

$\begin{array}{llllll}-10 & -5 & 3 & 66 & 63 & -27\end{array}$

$\begin{array}{llllll}-9 & -5 & 3 & 44 & -85 & -39\end{array}$

$\begin{array}{llllll}-8 & -5 & 3 & 122 & 142 & 13\end{array}$

$\begin{array}{llllll}-7 & -5 & 3 & 145 & -116 & 10\end{array}$

$\begin{array}{lllll}-5 & -5 & 3 & 254 & 231\end{array}$

$\begin{array}{lllll}-4 & -5 & 3 & 472 & 458\end{array}$

$-3 \quad-5 \quad 3 \quad 1068-1089$

$\begin{array}{rrrrr}-2 & -5 & 3 & 785 & 767 \\ -1 & -5 & 3 & 217 & -204\end{array}$

$\begin{array}{lllll}0 & -5 & 3 & 514 & 473\end{array}$

$\begin{array}{llllll}1 & -5 & 3 & 818 & -751\end{array}$

$\begin{array}{lllll}2 & -5 & 3 & 468 & 443\end{array}$

$\begin{array}{rrrrr}3 & -5 & 3 & 310 & -336 \\ 4 & -5 & 3 & 307 & 327\end{array}$

$\begin{array}{lllll}5 & -5 & 3 & 260 & -248\end{array}$

$\begin{array}{llllll}6 & -5 & 3 & 324 & 356 & 7\end{array}$

$\begin{array}{lllll}7 & -5 & 3 & 288 & -320\end{array}$

$\begin{array}{llllll}8 & -5 & 3 & 170 & 188 & 11\end{array}$

$\begin{array}{rrrrrr}9 & -5 & 3 & 115 & -169 & 16 \\ 10 & -5 & 3 & 37 & 39 & -43\end{array}$

$\begin{array}{llllll}-12 & -4 & 3 & 26 & -14 & -19\end{array}$

$\begin{array}{llllll}-11 & -4 & 3 & 42 & -21 & -33\end{array}$

$\begin{array}{llllll}-9 & -4 & 3 & 27 & -40 & -42\end{array}$

$\begin{array}{rrrrrr}-8 & -4 & 3 & 65 & -59 & -18 \\ -7 & -4 & 3 & 121 & 81 & 12\end{array}$

$\begin{array}{llllll}-6 & -4 & 3 & 103 & 46 & 11\end{array}$

$\begin{array}{rrrrrr}-5 & -4 & 3 & 76 & -50 & 13 \\ -4 & -4 & 3 & 387 & 370 & 6\end{array}$ $\begin{array}{rrrrrr}-3 & -4 & 3 & 116 & -70 & 8 \\ -2 & -4 & 3 & 387 & -367 & 5 \\ -1 & -4 & 3 & 82 & 29 & 10 \\ 0 & -4 & 3 & 462 & 417 & 5 \\ 1 & -4 & 3 & 284 & 250 & 5 \\ 2 & -4 & 3 & 363 & -354 & 6 \\ 3 & -4 & 3 & 138 & 154 & 8 \\ 4 & -4 & 3 & 106 & -70 & 10 \\ 5 & -4 & 3 & 172 & -165 & 8 \\ 6 & -4 & 3 & 190 & 160 & 8 \\ 7 & -4 & 3 & 116 & 17 & 12 \\ 8 & -4 & 3 & 102 & 8 & 15 \\ 9 & -4 & 3 & 13 & -16 & -53 \\ 10 & -4 & 3 & 62 & -9 & -31\end{array}$

$\begin{array}{llllll}-11 & 0 & 3 & 47 & 48 & -28\end{array}$

$\begin{array}{llllll}-10 & 0 & 3 & 59 & -25 & -21 \\ -9 & 0 & 3 & 93 & -79 & 2\end{array}$

$\begin{array}{llllll}-8 & 0 & 3 & 97 & 88 & 18\end{array}$

$\begin{array}{llllll}-7 & 0 & 3 & 104 & -49 & 13\end{array}$

$\begin{array}{lllll}-6 & 0 & 3 & 128 & 116\end{array}$

$\begin{array}{rrrrrr}-5 & 0 & 3 & 131 & 129 & 6 \\ -4 & 0 & 3 & 92 & 52\end{array}$

$\begin{array}{llllll}-3 & 0 & 3 & 257 & -289 & 3\end{array}$

$\begin{array}{llllll}-2 & 0 & 3 & 308 & -304 & 3\end{array}$

$\begin{array}{rrrrrr}-1 & 0 & 3 & 437 & -441 & 3 \\ 0 & 0 & 3 & 538 & 679 & -8\end{array}$

1035615982

$\begin{array}{llllll}11 & -4 & 3 & 38 & -30 & -43\end{array}$

$\begin{array}{rrrrrr}-12 & -3 & 3 & 32 & 18 & -44 \\ -11 & -3 & 3 & 52 & -19 & -31\end{array}$

$\begin{array}{llllll}-10 & -3 & 3 & 82 & -23 & -17\end{array}$

$\begin{array}{llllll}-9 & -3 & 3 & 110 & -120 & 15\end{array}$

$\begin{array}{llllll}-8 & -3 & 3 & 159 & 164 & 10\end{array}$

$\begin{array}{lllll}-7 & -3 & 3 & 277 & -298\end{array}$

$\begin{array}{rrrrr}-6 & -3 & 3 & 373 & 360 \\ -5 & -3 & 3 & 347 & -346\end{array}$

$\begin{array}{lllll}-4 & -3 & 3 & 452 & 439\end{array}$

$\begin{array}{llllll}-3 & -3 & 3 & 284 & -269\end{array}$

$\begin{array}{lllll}-2 & -3 & 3 & 805 & 846\end{array}$

$\begin{array}{rrrrr}-1 & -3 & 3 & 575 & -662 \\ 0 & -3 & 3 & 549 & 537\end{array}$

$\begin{array}{lllll}1 & -3 & 3 & 744 & -700\end{array}$

$\begin{array}{llllll}2 & -3 & 3 & 523 & 490 & 5\end{array}$

$\begin{array}{llllll}3 & -3 & 3 & 132 & -129 & 7\end{array}$

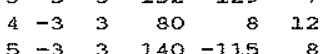

$\begin{array}{rrrrr}6 & -3 & 3 & 140 & -115 \\ 7 & 323 & 321 & 6\end{array}$

$\begin{array}{llllll}7 & -3 & 3 & 239 & -252 & 7\end{array}$

$\begin{array}{rrrrrr}8 & -3 & 3 & 184 & 199 & 10 \\ 9 & -3 & 3 & 113 & -151 & 15\end{array}$

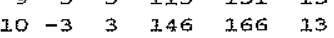

$\begin{array}{llllll}11 & -3 & 3 & 37 & -33 & -42\end{array}$

$\begin{array}{llllll}-12 & -2 & 3 & 43 & 41 & -30\end{array}$

$\begin{array}{llllll}-11 & -2 & 3 & 63 & -18 & -28\end{array}$

$\begin{array}{rrrrrr}-10 & -2 & 3 & 81 & 89 & -19 \\ -9 & -2 & 3 & 80 & -43 & -18\end{array}$

$\begin{array}{rrrrrr}-9 & -2 & 3 & 80 & -43 & -18 \\ -8 & -2 & 3 & 145 & -143 & 10\end{array}$

$\begin{array}{llllll}-7 & -2 & 3 & 144 & 97 & 8\end{array}$

$\begin{array}{llllll}-6 & -2 & 3 & 116 & -97 & 10\end{array}$

$\begin{array}{lllll}-5 & -2 & 3 & 166 & -157\end{array}$

$\begin{array}{lllll}-3 & -2 & 3 & 173 & 131 \\ -13 & 382 & -384\end{array}$

$\begin{array}{rrrrr}-2 & -2 & 3 & 219 & 247\end{array}$

$\begin{array}{lllll}-1 & -2 & 3 & 104 & 118\end{array}$

$\begin{array}{lllll}0 & -2 & 3 & 714 & 683\end{array}$

$\begin{array}{rrrrr}1 & -2 & 3 & 793 & -705 \\ 2 & -2 & 3 & 66 & -74\end{array}$

$\begin{array}{rrrrrr}2 & -2 & 3 & 66 & -74 & 12 \\ 3 & -2 & 3 & 310 & 308 & 5\end{array}$

$\begin{array}{lllll}4 & -2 & 3 & 463 & -426\end{array}$

$\begin{array}{llllll}5 & -2 & 3 & 84 & 81 & 11\end{array}$

$\begin{array}{rrrrrr}6 & -2 & 3 & 123 & -117 & 10 \\ 7 & -2 & 3 & 175 & 197 & 8\end{array}$

$\begin{array}{llllll}8 & -2 & 3 & 107 & -112 & 14\end{array}$

$\begin{array}{rrrrrr}10 & -2 & 3 & 58 & -8 & -31\end{array}$

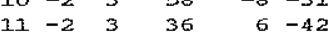

$\begin{array}{llllll}12 & -2 & 3 & 27 & 31 & -45\end{array}$

$\begin{array}{rrrrrr}-12 & -1 & 3 & 65 & 26 & -29 \\ -31 & -1 & 3 & 78 & -32 & -20\end{array}$

$\begin{array}{llllll}-10 & -1 & 3 & 92 & 118 & 16\end{array}$

$\begin{array}{llllll}-9 & -1 & 3 & 58 & -61 & -27\end{array}$

$\begin{array}{lllllr}-8 & -1 & 3 & 143 & 136 & 9\end{array}$

$\begin{array}{rrrrr}-7 & -1 & 3 & 88 & -95 \\ -6 & -1 & 3 & 294 & 294\end{array}$

$\begin{array}{llllll}-5 & -1 & 3 & 433 & -444\end{array}$

$\begin{array}{lllll}-4 & -1 & 3 & 319 & 306\end{array}$

$\begin{array}{lllll}-3 & -1 & 3 & 629 & -66.1\end{array}$

$\begin{array}{llllll}-2 & -1 & 3 & 226 & -238\end{array}$

$\begin{array}{lllll}1 & -1 & 3 & 198 & 174 \\ 0 & -1 & 3 & 743 & 774\end{array}$

$\begin{array}{llll}1 & -1 & 3 & 1074-1011\end{array}$

$\begin{array}{lllll}2 & -1 & 3 & 510 & 476\end{array}$

$\begin{array}{lllll}3 & -1 & 3 & 844 & -820\end{array}$

$\begin{array}{rrrrr}4 & -1 & 3 & 609 & 617 \\ 5 & -1 & 3 & 327 & -324\end{array}$

$\begin{array}{llll}5 & -1 & 3 & 117\end{array}$

$\begin{array}{llllll}7 & -1 & 3 & 1.86 & -199 \quad 8\end{array}$

$8-1 \quad 3 \quad I I \quad 42-44$

$\begin{array}{rrrrrr}9 & -1 & 3 & 79 & -47 & -17 \\ 10 & -1 & 3 & 211 & 94 & 15\end{array}$

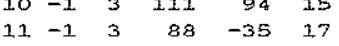

$\begin{array}{rrrrrr}12 & -1 & 3 & 81 & 43 & -27 \\ -12 & 0 & 3 & 64 & -6 & -20\end{array}$

$\begin{array}{llllll}2 & 0 & 3 & 256 & -234 & 3\end{array}$

$\begin{array}{rrrrrr}3 & 0 & 3 & 20 & 18 & -24 \\ 4 & 0 & 3 & 437 & -423 & 4\end{array}$

$\begin{array}{rrrrr}5 & 0 & 3 & 116 & 95 \\ 6 & 0 & 3 & 210 & 187\end{array}$

$700305 \quad 185 \quad 8$

$\begin{array}{rrrrrr}8 & 0 & 3 & 79 & -72 & 11 \\ 9 & 0 & 3 & 32 & -71 & -25\end{array}$

$\begin{array}{rlllll}9 & 0 & 3 & 32 & -71 & -25 \\ 20 & 0 & 3 & 87 & -61 & -28\end{array}$

$\begin{array}{llllll}11 & 0 & 3 & 94 & 72 & -22\end{array}$

$\begin{array}{llllll}13 & 0 & 3 & 68 & 23 & -22\end{array}$

$\begin{array}{llllll}-11 & 1 & 3 & 48 & -52 & -35\end{array}$

$\begin{array}{rrrrrr}-10 & 1 & 3 & 79 & 0 & -24 \\ -9 & 1 & 3 & 319 & -96 & 13\end{array}$

$\begin{array}{llllll}-3 & 1 & 3 & 62 & -46 & -25\end{array}$ 
h $\mathrm{k}$ I IOFO 1OFE IOS

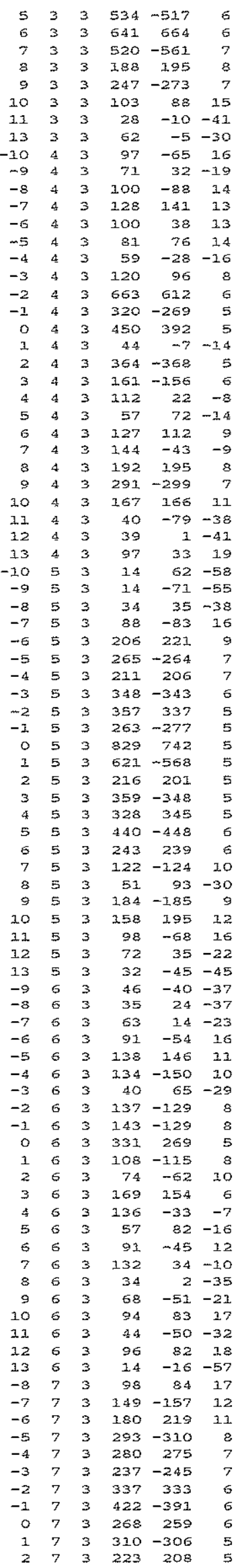

h $\mathrm{k} 1$ 10FO IOFC $10 \mathrm{~S}$

\begin{tabular}{|c|c|c|c|c|c|}
\hline 3 & 7 & 3 & 292 & -276 & 5 \\
\hline 4 & 7 & 3 & 436 & 459 & 6 \\
\hline 5 & 7 & 3 & 110 & -732 & 11 \\
\hline & 7 & 3 & 153 & 124 & 8 \\
\hline 7 & 7 & 3 & 1177 & 22 & 11 \\
\hline 8 & 7 & 3 & 58 & 45 & -20 \\
\hline 9 & 7 & 3 & 219 & -82 & 12 \\
\hline 10 & 7 & 3 & 45 & 88 & -35 \\
\hline 12 & 7 & 3 & 66 & -37 & -29 \\
\hline 13 & 7 & 3 & 85 & 6 & -22 \\
\hline 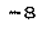 & 8 & 3 & 69 & -44 & -32 \\
\hline-7 & 8 & 3 & 58 & -15 & -34 \\
\hline-6 & 8 & 3 & 49 & 29 & -33 \\
\hline-5 & 8 & 3 & 37 & -33 & -39 \\
\hline-4 & 8 & 3 & 82 & -47 & -18 \\
\hline-3 & 8 & 3 & 77 & -23 & -1.6 \\
\hline-2 & 8 & 3 & 28 & 47 & -40 \\
\hline-1 & 8 & 3 & 43 & 26 & -29 \\
\hline 0 & 8 & 3 & 51. & -18 & -25 \\
\hline 1 & 8 & 3 & 83 & -99 & 13 \\
\hline$z$ & 8 & 3 & 81 & 75 & 11 \\
\hline 3 & 8 & 3 & 164 & -165 & 7 \\
\hline 4 & 8 & 3 & 94 & 62 & 12 \\
\hline 5 & 8 & 3 & 49 & -50 & -30 \\
\hline 6 & 8 & 3 & 85 & -12 & 13 \\
\hline 7 & 8 & 3 & 93 & 25 & 12 \\
\hline 8 & 8 & 3 & 42 & 41 & -34 \\
\hline 9 & 8 & 3 & 118 & -41 & 23 \\
\hline 10 & 8 & 3 & 65 & 53 & -28 \\
\hline 11 & 8 & 3 & 73 & -21 & -19 \\
\hline 12 & 8 & 3 & 4.1. & 40 & -38 \\
\hline 13 & 8 & 3 & 38 & 0 & -42 \\
\hline-7 & 9 & 3 & 86 & -69 & -19 \\
\hline-6 & 9 & 3 & 200 & 204 & 17 \\
\hline-5 & 9 & 3 & 141 & -168 & 14 \\
\hline-4 & 9 & 3 & 180 & 211 & 10 \\
\hline-3 & 9 & 3 & 178 & -195 & 10 \\
\hline-2 & 9 & 3 & 271 & 303 & 8 \\
\hline-1 & 9 & 3 & 286 & -303 & 8 \\
\hline 0 & 9 & 3 & 412 & 388 & 6 \\
\hline 1 & 9 & 3 & 284 & -271 & 6 \\
\hline 2 & 9 & 3 & 55 & 59 & -18 \\
\hline 3 & 9 & 3 & $\$ 2$ & -31 & -20 \\
\hline 4 & 9 & 3 & 332 & 353 & 6 \\
\hline 5 & 9 & 3 & 255 & -256 & 7 \\
\hline 6 & 9 & 3 & 196 & 192 & 8 \\
\hline 7 & 9 & 3 & 132 & -122 & 12 \\
\hline 8 & 9 & 3 & 73 & -47 & -19 \\
\hline 9 & 9 & 3 & 56 & -47 & -31 \\
\hline 20 & 9 & 3 & 61 & 34 & -29 \\
\hline 11 & 9 & 3 & 66 & -38 & -29 \\
\hline 12 & 9 & 3 & 61 & 15 & -34 \\
\hline 13 & 9 & 3 & 13 & -18 & -54 \\
\hline-6 & 10 & 3 & 33 & 19 & -45 \\
\hline-5 & 10 & 3 & 32 & -1 & -37 \\
\hline-4 & 10 & 3 & 89 & -44 & -24 \\
\hline-3 & 10 & 3 & 56 & 4 & -33 \\
\hline-2 & 10 & 3 & 95 & 117 & 16 \\
\hline-1 & 10 & 3 & 47 & -15 & -31 \\
\hline 0 & 10 & 3 & 98 & 75 & 15 \\
\hline 3 & 10 & 3 & 103 & 24 & 13 \\
\hline 2 & 10 & 3 & so & -23 & -33 \\
\hline 3 & 10 & 3 & 198 & -191 & 7 \\
\hline 4 & 10 & 3 & 41 & 84 & -32 \\
\hline 5 & 10 & 3 & 41 & 42 & -35 \\
\hline 6 & 10 & 3 & 93 & 56 & 17 \\
\hline 7 & 10 & 3 & 102 & -4 & 15 \\
\hline 8 & J. & 3 & 99 & 116 & 17 \\
\hline 9 & 10 & 3 & 131 & -130 & 14 \\
\hline 10 & 10 & 3 & 68 & 4 & -22 \\
\hline 11 & 10 & 3 & 55 & 20 & -35 \\
\hline 12 & 10 & 3 & 57 & -12 & -32 \\
\hline-5 & 11 & 3 & 132 & -147 & 15 \\
\hline-4 & 1.1 & 3 & 142 & 155 & 14 \\
\hline-3 & 11 & 3 & 167 & -199 & 12 \\
\hline-2 & 11 & 3 & 146 & 162 & 13 \\
\hline-1 & 11 & 3 & 165 & -159 & 11. \\
\hline 0 & 21 & 3 & $27 I$ & 298 & 9 \\
\hline 1 & 11 & 3 & 250 & -249 & 8 \\
\hline 2 & 11 & 3 & 193 & 206 & 10 \\
\hline 3 & 11 & .3 & 99 & -124 & 15 \\
\hline 4 & 11 & 3 & 109 & 122 & 22 \\
\hline 5 & 11 & 3 & 189 & -198 & 10 \\
\hline 6 & 3.1 & 3 & 164 & 159 & 12 \\
\hline 7 & 1.1 & 3 & 332 & -146 & 24 \\
\hline 8 & 21 & 3 & 1.22 & 124 & 17 \\
\hline 9 & 11 & 3 & 93 & -83 & -28 \\
\hline 10 & 22 & 3 & 64 & 97 & -25 \\
\hline 11 & 11 & 3 & 50 & -52 & -41 \\
\hline & & & 69 & & -25 \\
\hline
\end{tabular}

$\mathrm{h}$ k I 1OFO $10 \mathrm{FC}$ 1OS

$\begin{array}{llllll}-3 & 12 & 3 & 91 & -40 & -29\end{array}$ $\begin{array}{rrrrrr}-2 & 12 & 3 & 32 & 65 & -47 \\ -1 & 12 & 3 & 110 & -121 & 17\end{array}$

$\begin{array}{llllll}1 & 12 & 3 & 110 & -121 & 17 \\ 0 & 12 & 102 & 16\end{array}$

$\begin{array}{llllll}1 & 12 & 3 & 65 & -50 & -23\end{array}$

$\begin{array}{llllll}2 & 12 & 3 & 61 & 42 & -23\end{array}$

$\begin{array}{llllll}3 & 12 & 3 & 104 & -94 & 15\end{array}$

$\begin{array}{rrrrrr}4 & 12 & 3 & 98 & 70 & 12 \\ 5 & 12 & 3 & 36 & -13 & -37\end{array}$

$\begin{array}{llllll}5 & 12 & 3 & 85 & 23 & 17\end{array}$

$\begin{array}{rrrrrr}7 & 12 & 3 & 35 & -22 & -44 \\ 8 & 12 & 3 & 53 & 20 & -35\end{array}$

$\begin{array}{llllll}9 & 12 & 3 & 109 & -77 & 1.8\end{array}$

$\begin{array}{llllll}10 & 12 & 3 & 42 & 78 & -44\end{array}$

$\begin{array}{llllll}11 & 12 & 3 & 48 & -53 & -40\end{array}$

$\begin{array}{llllll}-2 & 13 & 3 & 149 & 158 & 15\end{array}$

$\begin{array}{llllll}-1 & 13 & 3 & 175 & -164 & 13 \\ 0 & 13 & 3 & 154 & 164 & 13\end{array}$

$\begin{array}{llllll}1 & 13 & 3 & 190 & -199 & 12\end{array}$

$\begin{array}{llllll}2 & 13 & 3 & 174 & 169 & 12\end{array}$

$\begin{array}{llllll}3 & 13 & 3 & 366 & -189 & 13\end{array}$

$\begin{array}{llllll}4 & 13 & 3 & 154 & 172 & 11 \\ 5 & 13 & 3 & 105 & -129 & 15\end{array}$

$\begin{array}{llllll}6 & 13 & 3 & 108 & 94 & 17\end{array}$

$\begin{array}{llllll}8 & 13 & 3 & 67 & 95 & -33\end{array}$

$\begin{array}{llllll}9 & 13 & 3 & 92 & -94 & -21\end{array}$

10 $13 \quad 3 \quad 51 \quad 51-43$

$\begin{array}{llll}0.14 & 55 & 89 & -36\end{array}$

$\begin{array}{rrrrrr}1 & 14 & 3 & 43 & -69 & -42 \\ 2 & 14 & 3 & 50 & 24 & -39\end{array}$

$\begin{array}{rrrrrr}2 & 14 & 3 & 50 & 24 & -39 \\ 3 & 14 & 3 & 52 & -69 & -38\end{array}$

$\begin{array}{llllrr}4 & 14 & 3 & 71 & 34 & -30\end{array}$

$\begin{array}{rrrrrr}5 & 14 & 3 & 45 & -19 & -34 \\ 6 & 14 & 3 & 49 & 43 & -41\end{array}$

$\begin{array}{llllll}7 & 14 & 3 & 82 & -30 & -23\end{array}$

$\begin{array}{rrrrrr}7 & 14 & 3 & 82 & -30 & -23 \\ 8 & 14 & 3 & 62 & 14 & -33\end{array}$

$\begin{array}{lllll}-7-13 & 4 & 41 & -38 & -43\end{array}$

$\begin{array}{rrrrr}-6-13 & 4 & 39 & 80 & -41 \\ -5-13 & 4 & 89 & -68 & 17\end{array}$

$\begin{array}{lllll}-4-13 & 4 & 51 & 50 & -33\end{array}$

$\begin{array}{lllll}-3-13 & 4 & 46 & -59 & -36\end{array}$

$-2-13 \quad 4 \quad 114 \quad 8316$

$\begin{array}{rrrrr}-1-13 & 4 & 87 & -75 & -20 \\ 0-13 & 4 & 64 & 66 & -24\end{array}$

$\begin{array}{lllll}1-13 & 4 & 39 & -76 & -47\end{array}$

$\begin{array}{lllll}-8-12 & 4 & 45 & 64 & -40\end{array}$

$\begin{array}{lllll}-7-12 & 4 & 150 & -132 & 12\end{array}$

$\begin{array}{rrrrr}-6-12 & 4 & 104 & 108 & 16 \\ -5-12 & 4 & 49 & -92 & -33\end{array}$

$\begin{array}{rrrrr}-5-12 & 4 & 49 & -92 & -33 \\ -4-12 & 4 & 88 & 11.5 & 17\end{array}$

$\begin{array}{lllll}-3-12 \quad 4 & 159 & -155 & 11\end{array}$

$\begin{array}{lllll}-2-12 & 4 & 139 & 134 & 12\end{array}$

$\begin{array}{lllll}-1-12 & 4 & 167 & -162 & 12\end{array}$

$\begin{array}{lllll}0-12 & 4 & 167 & 198 & 13 \\ 1-12 & 4 & 144 & -140 & 14\end{array}$

$\begin{array}{lllll}2-12 & 4 & 83 & 113 & -22\end{array}$

$\begin{array}{lllll}3-12 & 4 & 123 & -120 & 16\end{array}$

$\begin{array}{lllll}-9-11 & 4 & 49 & -3 & -29 \\ -8-11 & 4 & 70 & 13 & -28\end{array}$

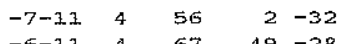

$\begin{array}{rrrrr}-6-11 & 4 & 67 & 49 & -28 \\ -5-11 & 4 & 926 & -114 & 12\end{array}$

$\begin{array}{lllll}-5-11 & 4 & 126 & -114 & 12 \\ -4-11 & 4 & 230 & 131 & 12\end{array}$

$\begin{array}{lllll}-3-11 & 4 & 215 & -113 & 14\end{array}$

$\begin{array}{llrrr}-2-11 & 4 & 32 & 42 & -32 \\ -1-11 & 4 & 128 & -104 & 12\end{array}$

$\begin{array}{lllll}0-11 & 4 & 69 & 44 & -21\end{array}$

$\begin{array}{lllll}1-11 & 4 & 101 & 26 & 14\end{array}$

$2411 \quad 4 \quad 34 \quad 400-34$

$\begin{array}{lllll}3-11 & 4 & 61 & -22 & -32 \\ 4-11 & 4 & 73 & -30 & -30\end{array}$

$\begin{array}{ccccc}-10-10 & 4 & 49 & 57 & -36\end{array}$

$\begin{array}{lllll}-9-10 & 4 & 93 & -56 & 18\end{array}$

$\begin{array}{lllll}-8-30 & 4 & 51 & -50 & -32\end{array}$

$\begin{array}{lllll}-7-30 & 4 & 78 & -53 & -19 \\ -6-30 & 4 & 347 & 382 & 12\end{array}$

$\begin{array}{lllll}-5-10 & 4 & 215 & -223 & 9\end{array}$

$\begin{array}{lllll}-4 m 10 & 4 & 176 & 183 & 20\end{array}$

$-3-10 \quad 4 \quad 139-151 \quad 31$

$\begin{array}{ccccc}-2-10 & 4 & 229 & 211 & 8\end{array}$

$\begin{array}{rlrrr}-1-10 & 4 & 269 & -279 & 8 \\ 0-10 & 4 & 206 & 204 & 10\end{array}$

$3-30 \quad 4 \quad 274 \quad-275 \quad 8$

$\begin{array}{lllll}2-10 & 4 & 220 & 224 & 10\end{array}$

$\begin{array}{lllll}3-10 & 4 & 87 & -88 & -18\end{array}$

$\begin{array}{rrrrr}4-10 & 4 & 136 & 144 & 35 \\ 5-10 & 4 & 128 & -170 & 16\end{array}$

$\begin{array}{rrrrr}5-10 & 4 & 128 & -170 & 16 \\ 6-10 & 4 & 38 & 93 & -43\end{array}$

$\begin{array}{llllll}-11 & -9 & 4 & 50 & -23 & -37\end{array}$ $k 1$ 10FO 1OFC $10 \mathrm{~s}$

$\begin{array}{rrrrrr}-8 & -9 & 4 & 99 & 59 & 14\end{array}$

$\begin{array}{llllll}-7 & -9 & 4 & 101 & -48 & 15\end{array}$

$\begin{array}{llllll}-5 & -9 & 4 & 164 & 170 & 10\end{array}$

$\begin{array}{llllll}-4 & -9 & 4 & 114 & -75 & 12\end{array}$

$\begin{array}{rrrrrr}-3 & -9 & 4 & 106 & -1.29 & 13 \\ -2 & -9 & 4 & 102 & 76 & 11\end{array}$

$\begin{array}{llllll}-1 & -9 & 4 & 63 & -13 & -19\end{array}$

$\begin{array}{llllll}0 & -9 & 4 & 55 & 53 & -28\end{array}$

$\begin{array}{llllll}1 & -9 & 4 & 78 & -42 & -18\end{array}$

$\begin{array}{llllll}2 & -9 & 4 & 87 & -65 & -21 \\ 3 & -9 & 4 & 74 & -12 & -19\end{array}$

$\begin{array}{llllll}4 & -9 & 4 & 12 & 7 & -47\end{array}$

$\begin{array}{llllll}5 & -9 & 4 & 121 & 116 & 14\end{array}$

$\begin{array}{llllll}6 & -9 & 4 & 26 & -42 & -51\end{array}$

$\begin{array}{rrrrrr}7 & -9 & 4 & 47 & -21 & -40 \\ -11 & -8 & 4 & 12 & -13 & -49\end{array}$

$\begin{array}{llllll}-10 & -8 & 4 & 43 & 20 & -37\end{array}$

$\begin{array}{llllll}-9 & -8 & 4 & 50 & -65 & -30\end{array}$

$\begin{array}{llllll}-8 & -8 & 4 & 12 & 30 & -49\end{array}$

$\begin{array}{rrrrrr}-7 & -8 & 4 & 104 & -124 & 15 \\ -6 & -8 & 4 & 158 & 157 & 10\end{array}$

$\begin{array}{rrrrrr}-5 & -8 & 4 & 358 & -376 & 10\end{array}$

$\begin{array}{lllllllllllll}-4 & -8 & 4 & 362 & 380 & 6\end{array}$

$\begin{array}{rrrrrr}-3 & -8 & 4 & 138 & -350 & 9 \\ -2 & -8 & 4 & 125 & 102 & 9\end{array}$

$\begin{array}{llllll}-1 & -8 & 4 & 225 & -222 & 7\end{array}$

$\begin{array}{llllll}0 & -8 & 4 & 241 & 240 & 7\end{array}$

$\begin{array}{rrrrrr}1 & -8 & 4 & 70 & -119 & -18 \\ 2 & -8 & 4 & 183 & 222 & 10 \\ 3 & -8 & 4 & 232 & -218 & 8\end{array}$

$\begin{array}{llllll}3 & -8 & 4 & 232 & -218 & 8\end{array}$

$\begin{array}{rrrrrr}4 & -8 & 4 & 1.60 & 154 & 11 \\ 5 & -8 & 4 & 130 & -1.23 & 13\end{array}$

$\begin{array}{llllll}6 & -8 & 4 & 130 & 177 & 14\end{array}$

$\begin{array}{llllll}7 & -8 & 4 & 45 & -67 & -38\end{array}$

$\begin{array}{llllll}8 & -8 & 4 & 74 & 60 & -22\end{array}$

$\begin{array}{llllll}-12 & -7 & 4 & 57 & 23 & -35 \\ -11 & -7 & 4 & 77 & -6 & -27\end{array}$

$\begin{array}{llllll}-10 & -7 & 4 & 68 & 23 & -29\end{array}$

$\begin{array}{llllll}-9 & -7 & 4 & 52 & -5 & -26\end{array}$

$\begin{array}{rrrrrr}-8 & -7 & 4 & 110 & -34 & 20 \\ -7 & -7 & 4 & 82 & 17 & -18\end{array}$

$\begin{array}{rrrrrr}-7 & -7 & 4 & 82 & 17 & -18 \\ -6 & -7 & 4 & 51 & 51 & -27\end{array}$

$\begin{array}{llllll}-5 & -7 & 4 & 157 & -133 & 8\end{array}$

$\begin{array}{rrrrrr}-4 & -7 & 4 & 216 & -209 & 7 \\ -3 & -7 & 4 & 98 & -113 & 10\end{array}$

$\begin{array}{rrrrrr}-3 & -7 & 4 & 98 & -1.13 & 10 \\ -2 & -7 & 4 & 149 & 156 & 7\end{array}$

$\begin{array}{rrrrrr}-1 & -7 & 4 & 62 & -27 & -16 \\ 0 & -7 & 4 & 54 & -4 & -17\end{array}$

$\begin{array}{llllll}1 & -7 & 4 & 62 & 30 & -24\end{array}$

$\begin{array}{llllll}2 & -7 & 4 & 24 & -62 & -40\end{array}$

$\begin{array}{llllll}3-7 & 4 & 80 & 81 & 15\end{array}$

$\begin{array}{llllll}4 & -7 & 4 & 120 & -108 & 12 \\ 5 & -7 & 4 & 102 & 106 & 14\end{array}$

$\begin{array}{llllll}6 & -7 & 4 & 104 & -63 & 15\end{array}$

$\begin{array}{llllll}7 & -7 & 4 & 68 & 105 & -22\end{array}$

$\begin{array}{rrrrrr}8 & -7 & 4 & 13 & -42 & -51 \\ 9 & -7 & 4 & 27 & 10 & -43\end{array}$

$\begin{array}{rrrrrr}9 & -7 & 4 & 27 & 10 & -43 \\ -12 & -6 & 4 & 59 & 15 & -35 \\ -11 & -6 & 4 & 75 & -38 & -25\end{array}$

$\begin{array}{rrrrrr}-11 & -6 & 4 & 76 & -38 & -25 \\ -30 & -6 & 4 & 109 & 100 & 1.4\end{array}$

$\begin{array}{llllll}-9 & -6 & 4 & 109 & 100 & 14 \\ -9 & -6 & 4 & 1.18 & \cdots 133 & 14\end{array}$

$\begin{array}{llllll}-8 & -6 & 4 & 1.58 & 153 & 11 \\ -7 & -6 & 4 & 119 & -87 & 11\end{array}$

$\begin{array}{rrrrrr}-7 & -6 & 4 & 119 & -87 & 11 \\ -6 & -6 & 4 & 146 & 341 & 9\end{array}$

$\begin{array}{llllll}-5 & -6 & 4 & 95 & -75 & 11\end{array}$

$\begin{array}{llllll}-4 & -6 & 4 & 334 & 344 & 5\end{array}$

$\begin{array}{rrrrr}-3 & -6 & 4 & 597 & -621 \\ -2 & -6 & 4 & 334 & 313\end{array}$

$\begin{array}{lllll}-1 & -6 & 4 & 361 & -373\end{array}$

$\begin{array}{lllll}0 & -6 & 4 & 582 & 579\end{array}$

$\begin{array}{lllll}1 & -6 & 4 & 298 & -291\end{array}$

$\begin{array}{lllll}2 & -6 & 4 & 317 & 315\end{array}$

$\begin{array}{lllll}3 & -6 & 4 & 200 & -219\end{array}$

$\begin{array}{rrrrr}4 & -6 & 4 & 275 & 282 \\ 5 & -6 & 4 & 230 & -236\end{array}$

$\begin{array}{llllll}6 & -6 & 4 & 176 & 201 & 13\end{array}$

$\begin{array}{llllll}7 & -6 & 4 & 123 & -150 & 15\end{array}$

$\begin{array}{llllll}8 & -6 & 4 & 33 & 99 & -51\end{array}$

$\begin{array}{rrrrrr}9 & -6 & 4 & 32 & -40 & -45 \\ 20 & -6 & 4 & 51 & 26 & -34\end{array}$

$\begin{array}{llllll}-12 & -5 & 4 & 32 & -8 & -41\end{array}$

$\begin{array}{llllll}-11 & -5 & 4 & 73 & -22 & -19\end{array}$

$\begin{array}{llllll}-10 & -5 & 4 & 44 & -27 & -37\end{array}$

$\begin{array}{rrrrrr}-9 & -5 & 4 & 31 & -73 & -42 \\ -8 & -5 & 4 & 55 & 47 & -28\end{array}$

$\begin{array}{lllllll}-7 & -5 & 4 & 52 & -53 & -29\end{array}$

$\begin{array}{llllll}-6 & -5 & 4 & 190 & 184 & 7\end{array}$

\begin{tabular}{|c|c|c|c|c|c|}
\hline & -5 & & 37 & 160 & \\
\hline & -5 & & & -103 & \\
\hline & -5 & & 221 & 206 & \\
\hline & -5 & & 02 & -288 & \\
\hline & -5 & & .72 & 175 & \\
\hline & -5 & & 80 & -49 & \\
\hline & 5 & & 53 & 55 & \\
\hline & & & 151 & -1立7 & \\
\hline & & & 85 & -7 & \\
\hline & - & & 202 & & \\
\hline & -5 & & 44 & 47 & \\
\hline & - & & 86 & -23 & \\
\hline & - & & 49 & 5 & -33 \\
\hline & - & & 84 & -28 & \\
\hline & -5 & & 49 & -11 & \\
\hline & -4 & & 79 & -4 & -20 \\
\hline &.- & & 46 & 44 & -35 \\
\hline & & & 37 & & \\
\hline & & & 80 & -75 & \\
\hline & -4 & & 12 & -5 & \\
\hline & -4 & & 177 & -206 & \\
\hline 6 & -4 & & 269 & 273 & \\
\hline & & & & -357 & \\
\hline & -4 & & 351 & 366 & \\
\hline & & & 388 & -393 & \\
\hline 2 & & & & 647 & \\
\hline & -4 & & 523 & -516 & \\
\hline & & & 964 & 929 & \\
\hline 1. & - & & $\mathrm{s}_{12}$ & -524 & \\
\hline 2 & & & 87 & 646 & \\
\hline & & & 552 & -577 & \\
\hline 4 & - & & 275 & 287 & \\
\hline 5 & & & 89 & -429 & \\
\hline 6 & -4 & & 301 & 318 & \\
\hline 7 & & & 162 & -160 & \\
\hline & & & 187 & 221 & \\
\hline 9 & -4 & & 125 & -1.62 & \\
\hline .0 & & & 89 & 125 & \\
\hline 1.1 & & & 53 & -78 & -28 \\
\hline 2 & -3 & & 56 & 1 & -33 \\
\hline & & & & 57 & \\
\hline & & & & & -2 \\
\hline 8 & -3 & & 25 & 27 & -4 \\
\hline & -3 & & 128 & 71 & 10 \\
\hline-6 & -3 & & 124 & -128 & \\
\hline-5 & -3 & & 64 & -14 & -18 \\
\hline & -3 & & 218 & -180 & \\
\hline & -3 & & 420 & -429 & \\
\hline-2 & -3 & & 126 & 152 & \\
\hline & -3 & & 399 & -393 & \\
\hline & & & & & \\
\hline 1 & & & & -138 & \\
\hline 2 & -3 & & 356 & 314 & \\
\hline 3 & & & & & \\
\hline & -3 & & 256 & 125 & \\
\hline 5 & -3 & & 161 & 1.72 & \\
\hline & & & 109 & -102 & \\
\hline & & & 111 & -63 & \\
\hline 8 & $m$ & & 9 & -50 & \\
\hline & - & & 73 & -9 & \\
\hline & - & & 64 & 44 & \\
\hline & - & & & -28 & -3 \\
\hline 2 & -2 & & 59 & 38 & -3 \\
\hline & -2 & & 25 & -24 & -4 \\
\hline & - & & & 32 & -2 \\
\hline-9 & -2 & & & -43 & \\
\hline 8 & - & & & & -3 \\
\hline & & & & -110 & \\
\hline-6 & -2 & & 327 & 342 & \\
\hline 5 & - & & & -214 & \\
\hline & - & & & 259 & \\
\hline-3 & - & & 459 & -487 & \\
\hline 2 & - & & & & \\
\hline 1 & - & & & -707 & \\
\hline & - & & 298 & 310 & \\
\hline 1 & - & 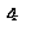 & 443 & -463 & \\
\hline 2 & - & & & 368 & \\
\hline & & & & -70 & \\
\hline & - & & 504 & 60 & \\
\hline 5 & -2 & 4 & 235 & -242 & \\
\hline & & & & 501 & \\
\hline & & & 27 & -85 & \\
\hline 8 & -2 & 4 & & & \\
\hline & & 4 & & -196 & \\
\hline & & & & 93 & \\
\hline & - & & & -79 & \\
\hline & & 4 & 7 & 56 & -2 \\
\hline & & & & -8 & \\
\hline & & & 2 & & \\
\hline
\end{tabular}


h $\mathrm{k}$ I $10 \mathrm{FO}$ IOFO $10 \mathrm{~s}$

$\begin{array}{llllll}-9 & -1 & 4 & 117 & -110 \quad 14\end{array}$ $\begin{array}{rrrrrr}-8 & -1 & 4 & 38 & 50 & -35 \\ -7 & -1 & 4 & 95 & -31 & 12\end{array}$

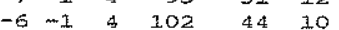
$\begin{array}{llllll}-5 & -1 & 4 & 30 & -76 & -24\end{array}$ $\begin{array}{rrrrrr}-4 & -1 & 4 & 69 & -32 & 13 \\ -3 & -1 & 4 & 76 & 32 & 11\end{array}$ $\begin{array}{llllll}-2 & -1 & 4 & 87 & -76 & 9\end{array}$ $\begin{array}{rrrrr}-1 & -1 & 4 & 77 & -36 \\ 0 & -1 & 4 & 844 & 848\end{array}$

$\begin{array}{lllll}1 & -1 & 4 & 335 & -325\end{array}$

$\begin{array}{rrrrrr}2 & -1 & 4 & 290 & 273 & 5 \\ 3 & -1 & 4 & 78 & -49 & 13\end{array}$

$\begin{array}{llllll}4 & -1 & 4 & 463 & -451 & 6\end{array}$

$\begin{array}{llllll}5 & -1 & 4 & 132 & 142 & 8\end{array}$

$\begin{array}{rrrrrr}6 & -1 & 4 & 94 & -58 & 12\end{array}$

$\begin{array}{rrrrrr}7 & -1 & 4 & 160 & 150 & 8 \\ 8 & -1 & 4 & 86 & -52 & 13\end{array}$

$9-1 \quad 1 \quad 109 \quad 105 \quad 12$

$\begin{array}{llllll}10 & -1 & 4 & 99 & -80 & 15\end{array}$

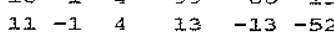

$\begin{array}{rrrrrr}12 & -1 & 4 & 58 & 38 & -33 \\ -12 & 0 & 4 & 53 & 31 & -21\end{array}$

$\begin{array}{llllll}-11 & 0 & 4 & 80 & -30 & -20\end{array}$

$\begin{array}{rrrrrr}-10 & 0 & 4 & 55 & 36 & -20 \\ -9 & 0 & 4 & 140 & -127 & -28\end{array}$

$\begin{array}{rrrrrr}-9 & 0 & 4 & 140 & -127 & -28 \\ -8 & 0 & 4 & 49 & -9 & -22\end{array}$

$\begin{array}{llllrr}-7 & 0 & 4 & 205 & -202 & 6 \\ -6 & 0 & 4 & 301 & 306 & 5\end{array}$

$\begin{array}{rrrrrr}-5 & 0 & 4 & 144 & -383 & 9 \\ -4 & 0 & 4 & 180 & 177 & 10\end{array}$

$\begin{array}{rlllrr}-4 & 0 & 4 & 180 & 177 & 1 \\ -3 & 0 & 4 & 446 & -460 & \end{array}$

$\begin{array}{llllll}-2 & 0 & 4 & 390 & 398 & 3\end{array}$

$0 \quad 04747852$

$\begin{array}{rrrrrr}1 & 0 & 4 & 495 & 487 & 3 \\ 2 & 0 & 4 & 1265 & 1242 & 16\end{array}$

$304 \quad 361-373 \quad 3$

404735714

$\begin{array}{llllll}5 & 0 & 4 & 473 & -438 & 4 \\ 6 & 0 & 4 & 414 & 409 & 5\end{array}$

$\begin{array}{rrrrrr}6 & 0 & 4 & 414 & 409 & 5 \\ 7 & 0 & 4 & 247 & -253 & 5\end{array}$

$\begin{array}{rrrrrr}8 & 0 & 4 & 147 & 120 & 8 \\ 9 & 0 & 4 & 68 & -65 & -14\end{array}$

$10 \quad 4 \quad 92 \quad 106 \quad 12$

$110460-29-20$

$\begin{array}{llllll}12 & 0 & 4 & 25 & 10 & -31 \\ 13 & 0 & 4 & 52 & 15 & -24\end{array}$

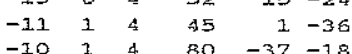

$\begin{array}{rrrrrr}-9 & 1 & 4 & 50 & 22 & -25\end{array}$

$\begin{array}{llllll}-8 & 1 & 4 & 89 & -95 & 16\end{array}$

$\begin{array}{rrrrrr}-7 & 1 & 4 & 91 & -86 & 14 \\ -6 & 1 & 4 & 46 & 49 & -21\end{array}$

$\begin{array}{lllllrr}-5 & 1 & 4 & 186 & -184 & 7\end{array}$

$\begin{array}{lllll}-4 & 1 & 4 & 172 & 171\end{array}$

$\begin{array}{lllll}-3 & 3 & 4 & 126 & -137 \\ -2 & 1 & 4 & 153 & -163\end{array}$

-1. 1126143

$\begin{array}{rrrrr}0 & 1 & 4 & 525 & -538 \\ 1 & 1 & 4 & 542 & 580\end{array}$

$\begin{array}{llllll}2 & 1 & 4 & 24 & -47 & -31\end{array}$

$\begin{array}{rrrrrr}3 & 1 & 4 & 492 & 460 & 5 \\ 4 & 1 & 493 & 99 & -5\end{array}$

$\begin{array}{rrrrrr}5 & 1 & 4 & 297 & 220 & 6 \\ 6 & 1 & 4 & 75 & 18 & 12\end{array}$

$\begin{array}{lllll}7 & 1 & 4 & 61 & 12\end{array}$

$\begin{array}{llllll}8 & 1 & 4 & 136 & -153 & 10 \\ 9 & 1 & 4 & 125 & -1.42 & 11\end{array}$

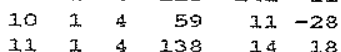

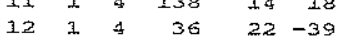

$\begin{array}{llllll}13 & 1 & 4 & 38 & 2 & -42\end{array}$

$\begin{array}{rrrrrr}-11 & 2 & 4 & 13 & -37 & -50 \\ -10 & 2 & 4 & 11 & 76 & -43\end{array}$

$\begin{array}{llllll}-9 & 2 & 4 & 125 & -128 & 14 \\ -8 & 2 & 4 & 110 & 103 & 14\end{array}$

$\begin{array}{llllll}-7 & 2 & 4 & 44 & -42 & -28\end{array}$

$\begin{array}{llllll}-6 & 2 & 4 & 195 & 192 & 7\end{array}$

$\begin{array}{lllll}-5 & 2 & 4 & 254 & -268\end{array}$

$\begin{array}{rrrrr}-4 & 2 & 4 & 326 & 308 \\ -3 & 2 & 4 & 370 & -347\end{array}$

$\begin{array}{lllll}-2 & 2 & 489 & 754\end{array}$

$\begin{array}{lllll}-1 & 2 & 4 & 665 & -685\end{array}$

- 2413801346

$\begin{array}{rrrrrr}1 & 2 & 4 & 753 & -771 & 4 \\ 2 & 2 & 4 & 79 & -34 & 10\end{array}$

$\begin{array}{llllll}3 & 2 & 4 & 32 & -59 & -28\end{array}$

$\begin{array}{rrrrrr}4 & 2 & 4 & 167 & 144 & 6 \\ 5 & 2 & 4 & 168 & -152 & 6\end{array}$

\section{h $\mathrm{k}$ l IOFO IOFC IOS}

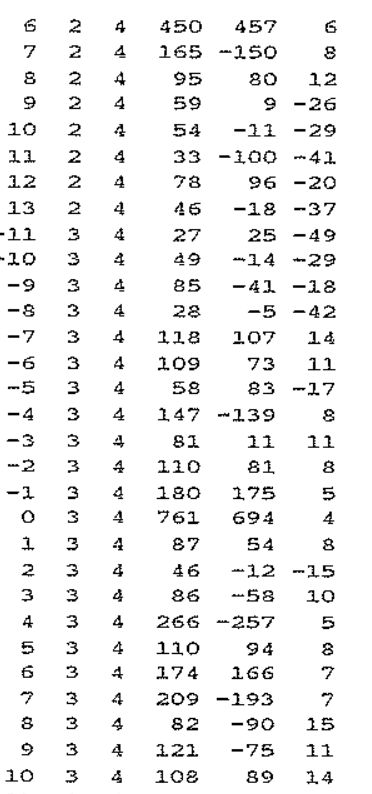

$\begin{array}{rrrrrr}10 & 3 & 4 & 108 & 89 & 14 \\ 11 & 3 & 4 & 13 & 33 & -50\end{array}$

$\begin{array}{llllll}12 & 3 & 4 & 30 & 5 & -42 \\ 13 & 3 & 4 & 48 & 2 & -30\end{array}$

$\begin{array}{lllll}13 & 4 & 4 & -11 & -35\end{array}$

$\begin{array}{rrrrrr}-9 & 4 & 4 & 39 & -42 & -37 \\ -8 & 4 & 4 & 23 & 62 & -46\end{array}$

$\begin{array}{rrrrrr}-8 & 4 & 4 & 23 & 62 & -4 \\ -7 & 4 & 4 & 214 & -225 & \end{array}$

$\begin{array}{rrrrr}-5 & 4 & 4 & 355 & 378 \\ -5 & 4 & 4 & 278 & -267\end{array}$

$\begin{array}{llllll}-4 & 4 & 4 & 344 & 344 & 5\end{array}$

$\begin{array}{rrrrr}-3 & 4 & 4 & 397 & -431 \\ -2 & 4 & 4 & 552 & 514\end{array}$

$\begin{array}{lllll}-1 & 4 & 4 & 599 & -542 \\ 0 & 4 & 578 & 535\end{array}$

$\begin{array}{lllll}1 & 4 & 4 & 706 & -671\end{array}$

$244676 \quad 670$

$\begin{array}{rrrr}34 & 4 & 619 & -620 \\ 4 & 4 & 185 & 196\end{array}$

$544116-124$ 8

644152 151

$\begin{array}{rrrrrr}7 & 4 & 4 & 172 & -131 & 7 \\ 8 & 4 & 4 & 227 & 229 & 7\end{array}$

$\begin{array}{rrrrrr}8 & 4 & 4 & 227 & 229 & 7 \\ 9 & 4 & 4 & 323 & -340 & 7\end{array}$

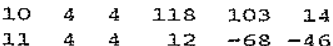

$\begin{array}{rrrrrr}12 & 4 & 4 & 80 & 44 & -17\end{array}$

$\begin{array}{llllll}13 & 4 & 4 & 57 & -1 & -27\end{array}$

$\begin{array}{rrrrrr}-10 & 5 & 4 & 63 & -32 & -27 \\ -9 & 5 & 4 & 49 & 22 & -28\end{array}$

$\begin{array}{rrrrrr}-9 & 5 & 4 & 49 & 22 & -28 \\ -8 & 5 & 4 & 71 & -92 & -21\end{array}$

$\begin{array}{llllll}-7 & 5 & 4 & 151 & 169 & 11\end{array}$

$\begin{array}{rrrrrr}-6 & 5 & 4 & 160 & -143 & 11 \\ -5 & 5 & 4 & 70 & -52 & -24\end{array}$

$\begin{array}{llllll}-4 & 5 & 4 & 94 & -35 & 12 \\ -3 & 5 & 9 & 49 & 19 & -24\end{array}$

$\begin{array}{rrrrrr}-2 & 5 & 4 & 105 & 92 & 9\end{array}$

$\begin{array}{rrrrr}-1 & 5 & 4 & 132 & 110 \\ 0 & 5 & 4 & 804 & 705\end{array}$

$\begin{array}{rrrrr}0 & 5 & 4 & 804 & 705 \\ 1 & 5 & 4 & 482 & -419\end{array}$

$\begin{array}{lllll}2 & 5 & 4 & 213 & 209 \\ 3 & 5 & 4 & 165 & 129\end{array}$

$4 \quad 5 \quad 4 \quad 259263 \quad 5$

$\begin{array}{rrrrrr}5 & 5 & 4 & 66 & -47 & -14 \\ 6 & 5 & 4 & 143 & 150 & 8\end{array}$

$\begin{array}{rrrrrr}7 & 5 & 4 & 115 & 47 & 10 \\ 8 & 5 & 4 & 163 & -172 & 9\end{array}$

$954 \quad 117$ 90 12

$\begin{array}{llllll}10 & 5 & 4 & 191 & 212 & 10\end{array}$

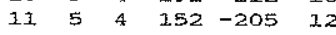

$\begin{array}{rrrrrr}12 & 5 & 4 & 95 & 130 & 19 \\ 13 & 5 & 4 & 59 & -47 & -27\end{array}$

$\begin{array}{llllll}-9 & 6 & 4 & 95 & -113 & -20\end{array}$

$\begin{array}{rrrrrr}-8 & 6 & 4 & 35 & 93 & -41 \\ -7 & 6 & 4 & 116 & -106 & 14\end{array}$

$\begin{array}{llllll}-7 & 6 & 4 & 116 & -106 & 24 \\ -6 & 6 & 4 & 177 & 194 & 10\end{array}$

$\begin{array}{rrrrrr}-5 & 6 & 4 & 124 & -147 & 12 \\ -4 & 6 & 4 & 58 & -9 & -25\end{array}$

$\begin{array}{llllll}-3 & 6 & 4 & 249 & -262 & 6\end{array}$

$\begin{array}{rrrrrr}-2 & 6 & 4 & 265 & 267 & 6 \\ -1 & 6 & 4 & 626 & -566 & 6\end{array}$ h k I loFO 1OFE $10 \mathrm{~s}$

$7104204-194 \quad 10$ $\begin{array}{rrrrrr}0 & 6 & 4 & 568 & 513 & 6 \\ 1 & 6 & 4 & 463 & -439 & 5 \\ 2 & 6 & 4 & 492 & 462 & 5 \\ 3 & 6 & 4 & 621 & -603 & 5 \\ 4 & 6 & 4 & 473 & 486 & 6 \\ 5 & 6 & 4 & 161 & -185 & 7 \\ 6 & 6 & 4 & 287 & 304 & 6 \\ 7 & 6 & 4 & 141 & -136 & 9 \\ 8 & 6 & 4 & 37 & 7 & -26 \\ 9 & 6 & 4 & 39 & -19 & -35 \\ 10 & 6 & 4 & 41 & 2 & -35 \\ 11 & 6 & 4 & 12 & 4 & -49 \\ 12 & 6 & 4 & 110 & 86 & 15 \\ 13 & 6 & 4 & 115 & -77 & 16 \\ -8 & 7 & 4 & 76 & -17 & -28 \\ -7 & 7 & 4 & 63 & -18 & -28 \\ -6 & 7 & 4 & 89 & 67 & 16 \\ -5 & 7 & 4 & 31 & -37 & -40 \\ -4 & 7 & 4 & 59 & 22 & -26 \\ -3 & 7 & 4 & 60 & 101 & -25 \\ -2 & 7 & 4 & 27 & -29 & -36 \\ -1 & 7 & 4 & 304 & 300 & 6 \\ 0 & 7 & 4 & 40 & 49 & -28 \\ 1 & 7 & 4 & 197 & -176 & 6 \\ 2 & 7 & 4 & 135 & 125 & 7 \\ 3 & 7 & 4 & 78 & 22 & 11 \\ 4 & 7 & 4 & 105 & 43 & 10 \\ 5 & 7 & 4 & 30 & 31 & -36 \\ 6 & 7 & 4 & 148 & 121 & 9 \\ 7 & 7 & 4 & 132 & -135 & 10 \\ 8 & 7 & 4 & 85 & 22 & 24 \\ 9 & 7 & 4 & 71 & -10 & -19 \\ 10 & 7 & 4 & 68 & 7 & -20 \\ 11 & 7 & 4 & 82 & 23 & -18 \\ 12 & 7 & 4 & 66 & -9 & -28 \\ 13 & 7 & 4 & 44 & 4 & -32 \\ -7 & 8 & 4 & 64 & -90 & -31 \\ -6 & 8 & 4 & 165 & 171 & 11\end{array}$

$\begin{array}{lllll}10 & 4 & 66 & 93 & -30\end{array}$

$\begin{array}{llllll}9 & 10 & 4 & 33 & -57 & -44\end{array}$

$\begin{array}{llllll}10 & 10 & 4 & 77 & 37 & -21\end{array}$

$\begin{array}{llllll}11 & 10 & 4 & 44 & -18 & -38\end{array}$

$\begin{array}{llllll}12 & 10 & 4 & 38 & 18 & -42 \\ -5 & 11 & 4 & 70 & 11 & -33\end{array}$

$\begin{array}{llllll}-4 & 12 & 4 & 26 & 27 & -47\end{array}$

$\begin{array}{llllll}-3 & 11 & 4 & 13 & 29 & -50\end{array}$

$\begin{array}{rrrrrr}-2 & 111 & 4 & 24 & -10 & -45 \\ -1 & 11 & 4 & 89 & -77 & 17\end{array}$

$\begin{array}{llllll}1 & 11 & 4 & 95 & -32 & 15\end{array}$

$\begin{array}{llllll}2 & 11 & 4 & 78 & 39 & -18\end{array}$

$\begin{array}{rrrrrr}3 & 11 & 4 & 11 & -9 & -43 \\ 4 & 11 & 4 & 37 & -54 & -35\end{array}$

$\begin{array}{llllll}4 & 11 . & 4 & 37 & -54 & -35 \\ 5 & 11 & 4 & 47 & -10 & -32\end{array}$

$611438 \quad 13-36$

$\begin{array}{llllll}7 & 11 & 4 & 50 & -33 & -28\end{array}$

$\begin{array}{llllll}8 & 12 & 4 & 69 & 49 & -29\end{array}$

$\begin{array}{rrrrrr}9 & 11 & 4 & 113 & -68 & 15 \\ 10 & 11 & 4 & 98 & 68 & 19\end{array}$

$\begin{array}{llllll}11 & 11 & 4 & 49 & 4 & -42\end{array}$

$\begin{array}{llllll}12 & 11 & 4 & 58 & 12 & -28\end{array}$

$\begin{array}{llllll}-3 & 12 & 4 & 73 & -137 & -32\end{array}$

$\begin{array}{rrrrrr}-2 & 12 & 4 & 165 & 188 & 14 \\ -1 & 12 & 4 & 138 & -134 & 13\end{array}$

$\begin{array}{llllll}1 & 12 & 4 & 170 & 177 & 12\end{array}$

$1124249-239 \quad 9$

$2124253 \quad 255$ 9

$\begin{array}{llllrr}3 & 12 & 4 & 251 & -234 & 8 \\ 4 & 12 & 4 & 194 & 202 & 8\end{array}$

$\begin{array}{llllll}5 & 12 & 4 & 139 & -147 & 11\end{array}$

$\begin{array}{llllll}612 & 4 & 67 & 73 & -22\end{array}$

$\begin{array}{lllllll}7 & 12 & 4 & 54 & -56 & -35\end{array}$

$\begin{array}{llllll}8 & 12 & 4 & 66 & 43 & -24\end{array}$

$\begin{array}{rrrrrr}912 & 4 & 114 & -105 & 16 \\ 10 & 12 & 4 & 104 & 79 & 18\end{array}$

$1112463 \quad-75-29$

$\begin{array}{llllll}-2 & 13 & 4 & 39 & 25 & -42\end{array}$

$\begin{array}{llllll}-13 & 13 & 38 & -53 & -46\end{array}$

$\begin{array}{rrrrrr}0 & 13 & 4 & 138 & 116 & 13 \\ 1 & 13 & 4 & 57 & -12 & -35\end{array}$

$\begin{array}{llllll}213 & 4 & 62 & -5 & -28\end{array}$

$\begin{array}{lllll}3 & 13 & 4 & -53 & -38\end{array}$

$\begin{array}{lllll}43 & 12 & 103 & -47\end{array}$

$\begin{array}{rrrrrr}5 & 13 & 4 & 83 & -75 & -18 \\ 7 & 13 & 4 & 47 & -7 & -38\end{array}$

$\begin{array}{lllll}8 & 13 & 4 & 71 & 3\end{array}$

$\begin{array}{llllll}9 & 13 & 4 & 27 & -29 & -50\end{array}$

$1013 \& 14 \quad 55-57$

$114 \& 149-160 \quad 14$

$2144201 \quad 223 \quad 12$

$\begin{array}{llllll}3 & 14 & 4 & 145 & -169 & 15\end{array}$

$\begin{array}{llllll}4 & 14 & 4 & 92 & 126 & 18\end{array}$

$\begin{array}{llllll}5 & 14 & 4 & 78 & -117 & -19 \\ 7 & 47 & 132 & -40\end{array}$

$\begin{array}{rrrrrr}7 & 14 & 4 & 82 & -67 & -23 \\ 8 & 14 & 4 & 77 & -7 & -31\end{array}$

$-6-135 \quad 66 \quad 46-22$

$\begin{array}{lllll}-5-13 & 5 & 55 & -49 & -32\end{array}$

$-4-13 \quad 5 \quad 49 \quad 31-34$

$\begin{array}{lllll}-3-13 & 5 & 75 & -159 & -32\end{array}$

$\begin{array}{lllll}-2-13 & 5 & 1.78 & 159 & 12\end{array}$

$\begin{array}{lllll}-1-13 \quad 5 \quad 82 & -129 & -23\end{array}$

$\begin{array}{rrrrr}0-13 & 5 & 153 & 149 & 13 \\ -8-12 & 5 & 66 & 67 & -32\end{array}$

$\begin{array}{rrrrr}-8-12 & 5 & 66 & 67 & -32 \\ -7-12 & 5 & 95 & -43 & 18\end{array}$

$\begin{array}{lllll}-5-12 & 5 & 54 & 52 & -32\end{array}$

$\begin{array}{lllll}-5-12 & 5 & 29 & -52 & -44 \\ -3-12 & 5 & 13 & -39 & -50\end{array}$

$\begin{array}{lllll}-2-12 & 5 & 75 & 39 & -20\end{array}$

$\begin{array}{lllll}-1-12 & 5 & 30 & -87 & -45\end{array}$

$\begin{array}{lllll}0-12 & 5 & 97 & 82 & 18\end{array}$

$\begin{array}{lllll}1-12 & 5 & 93 & -65 & -19\end{array}$

$\begin{array}{lllll}2-12 & 5 & 91 & 80 & -20\end{array}$

$\begin{array}{rrrrr}-9-11 & 5 & 55 & 15 & -33 \\ -8-11 & 5 & 47 & 2 & -35\end{array}$

$\begin{array}{rrrrr}-8-11 & 5 & 47 & 2 & -35 \\ -7-11 & 5 & 111 & -102 & 16\end{array}$

$\begin{array}{rrrrr}-6-11 & 5 & 218 & 247 & 10\end{array}$

$\begin{array}{lllll}-5-11 & 5 & 192 & -205 & 10\end{array}$

$\begin{array}{lllll}-3-11 & 5 & 168 & -176 & 10\end{array}$

$\begin{array}{lllll}-2-11 & 5 & 140 & 137 & 12\end{array}$

$\begin{array}{lllll}-1-11 & 5 & 159 & -147 & 11\end{array}$

$\begin{array}{lllll}0-11 & 5 & 249 & 175 & 12\end{array}$

$\begin{array}{lllll}1-11 & 5 & 213 & -214 & 10\end{array}$

$2-11 \quad 5 \quad 159 \quad 148 \quad 13$

$\begin{array}{lllll}3-11 & 5 & 133 & -136 & 14\end{array}$

$\begin{array}{rrrrrr}3 & 10 & 4 & 214 & -219 & 8 \\ 4 & 10 & 4 & 187 & 211 & 8\end{array}$

$\begin{array}{rrrrrr}5 & 10 & 4 & 153 & -179 & 31 \\ 6 & 10 & 4 & 234 & 237 & 9\end{array}$ h 1 IOFO $10 F \mathrm{OC}$ los

$\begin{array}{lllll}-9-10 & 5 & 36 & -31 & -41\end{array}$

$\begin{array}{lllll}-10 & 5 & 48 & -9 & -35\end{array}$

$\begin{array}{lllll}-7-10 & 5 & 28 & 28 & -43\end{array}$

$\begin{array}{rrrrr}-5-10 & 5 & 100 & -77 & 15 \\ -5-10 & 5 & 73 & -14 & -18\end{array}$

$-4-10 \quad 5 \quad 168 \quad 178 \quad 30$

$-3-10 \quad 5 \quad 99-105 \quad 14$

$-2-10 \quad 5 \quad 73 \quad 91-19$

$-1-10 \quad 5 \quad 13 \quad-68-51$

$\begin{array}{rrrrr}0-10 & 5 & 111 & 1.5 & 14 \\ 1-10 & 5 & 11.8 & -1.29 & 15\end{array}$

$\begin{array}{lllll}2-10 & 5 & 42 & 42 & -37\end{array}$

$\begin{array}{lllll}3-10 & 5 & 57 & -17 & -28\end{array}$

$\begin{array}{lllll}4-10 & 5 & 74 & -32 & -22\end{array}$

$\begin{array}{rrrrr}5-10 & 5 & 56 & 19 & -30 \\ 6-10 & 5 & 83 & -12 & -20\end{array}$

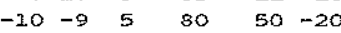

$\begin{array}{llllll}-9 & -9 & 5 & 89 & -103 & -19\end{array}$

$\begin{array}{rrrrrr}-8 & -9 & 5 & 83 & 94 & -18 \\ -7 & -9 & 5 & 47 & -60 & -32\end{array}$

$\begin{array}{llllll}-6 & -9 & 5 & 12 & 88 & -48\end{array}$

$\begin{array}{llllll}-5 & -9 & 5 & 147 & -171 & 11\end{array}$

$\begin{array}{llllll}-4 & -9 & 5 & 94 & 76 & 14\end{array}$

$\begin{array}{rrrrrr}-3 & -9 & 5 & 274 & -286 & 7 \\ -2 & -9 & 5 & 332 & 329 & 7\end{array}$

$\begin{array}{lllll}-1 & -9 & 5 & 275 & -265\end{array}$

$\begin{array}{lllllr}0 & -9 & 5 & 262 & 280 & 8\end{array}$

$\begin{array}{llllll}2 & -9 & 5 & 193 & 214 & 21\end{array}$

$\begin{array}{llllll}3 & -9 & 5 & 238 & -259 & 9\end{array}$

$\begin{array}{rrrrrr}4 & -9 & 5 & 173 & 193 & 12 \\ 5 & -9 & 5 & 135 & -157 & 15\end{array}$

$\begin{array}{llllll}6 & -9 & 5 & 71 & 117 & -31\end{array}$

$\begin{array}{lllll}7-9 & 5 & 54 & -93 & -29\end{array}$

$\begin{array}{llllll}-11 & -8 & 5 & 53 & 11 & -38\end{array}$

$\begin{array}{rrrrrr}-10 & -8 & 5 & 59 & -8 & -29 \\ -9 & -8 & 5 & 53 & 32 & -34\end{array}$

$\begin{array}{llllll}-8 & -8 & 5 & 36 & 11 & -37\end{array}$

$\begin{array}{llllll}-7 & -8 & 5 & 108 & -96 & 14\end{array}$

$\begin{array}{llllll}-6 & -8 & 5 & 181 & 199 & 10\end{array}$

$\begin{array}{rrrrrr}-5 & -8 & 5 & 53 & -10 & -23 \\ -4 & -8 & 5 & 74 & 11 & 15\end{array}$

$\begin{array}{llllll}-3 & -8 & 5 & 54 & -20 & -24\end{array}$

$\begin{array}{lllllll}-2 & -8 & 5 & 123 & -148 & 10\end{array}$

$\begin{array}{llllll}-1 & -8 & 5 & 104 & -88 & 13\end{array}$

$\begin{array}{rrrrrr}0 & -8 & 5 & 327 & 179 & 11 \\ 3 & -8 & 5 & 137 & -126 & 10\end{array}$

$\begin{array}{llllll}2 & -8 & 5 & 69 & 72 & -19\end{array}$

$\begin{array}{llllll}3 & -8 & 5 & 34 & 50 & -36\end{array}$

$\begin{array}{llllll}4 & -8 & 5 & 30 & -23 & -34\end{array}$

$\begin{array}{rrrrrr}5 & -8 & 5 & 84 & 15 & -18 \\ 6 & -8 & 5 & 49 & 7 & -26\end{array}$

$\begin{array}{llllll}7 & -8 & 5 & 55 & 68 & -27\end{array}$

$\begin{array}{llllll}8 & -8 & 5 & 61 & -56 & -31\end{array}$

$\begin{array}{llllll}-11 & -7 & 5 & 66 & -46 & -34\end{array}$

$\begin{array}{rrrrrr}-10 & -7 & 5 & 46 & 49 & -33 \\ -9 & -7 & 5 & 13 & -73 & -51\end{array}$

$\begin{array}{rrrrrr}-9 & -7 & 5 & 13 & -73 & -51 \\ -8 & -7 & 5 & 47 & 56 & -31\end{array}$

$\begin{array}{llllll}-7 & -7 & 5 & 51 & -49 & -30\end{array}$

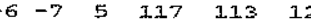

$\begin{array}{llllll}-5 & -7 & 5 & 112 & -132 & 11\end{array}$

$\begin{array}{rrrrrr}-4 & -7 & 5 & 280 & 303 & 6 \\ -3 & -7 & 5 & 142 & -144 & 8\end{array}$

$\begin{array}{llllll}-2 & -7 & 5 & 109 & 123 & 10\end{array}$

$\begin{array}{lllll}-2 & -7 & 5 & 252 & -234\end{array}$

$\begin{array}{lllll}0 & -7 & 5 & 389 & 375\end{array}$

$\begin{array}{rrrrr}1 & -7 & 5 & 358 & -348 \\ 2 & -7 & 5 & 225 & 225\end{array}$

$\begin{array}{llllll}3 & -7 & 5 & 233 & -215\end{array}$

$\begin{array}{llllll}4 & -7 & 5 & 134 & 122 & 12\end{array}$

$5-7 \quad 5 \quad 384-184 \quad 10$

$\begin{array}{llllll}6 & -7 & 5 & 199 & 208 & 10\end{array}$

$\begin{array}{rrrrrr}7 & -7 & 5 & 79 & -86 & -20 \\ 8 & -7 & 5 & 111 & 85 & 16\end{array}$

$\begin{array}{llllll}9 & -7 & 5 & 89 & -46 & -19\end{array}$

$\begin{array}{llllll}-12 & -6 & 5 & 76 & 20 & -28\end{array}$

$\begin{array}{llllll}-11 & -6 & 5 & 51 & -19 & -34\end{array}$

$\begin{array}{rrrrrr}-10 & -6 & 5 & 63 & 18 & -22 \\ -9 & -6 & 5 & 42 & -24 & -33\end{array}$

$\begin{array}{llllll}-8 & -6 & 5 & 91 & -28 & 15\end{array}$

$\begin{array}{llllll}-7 & -6 & 5 & 67 & 3 & -18\end{array}$

$\begin{array}{rrrrrr}-5 & -6 & 5 & 91 & 91 & 13 \\ -5 & -6 & 5 & 98 & -83 & 11\end{array}$

$\begin{array}{rrrrrr}-4 & -6 & 5 & 144 & 176 & 8\end{array}$

$\begin{array}{llllll}-3 & -6 & 5 & 96 & -110 & 20 \\ -2 & -6 & 5 & 98 & 94 & 20\end{array}$

$\begin{array}{llllll}-1 & -6 & 5 & 72 & -10 & 12\end{array}$

$\begin{array}{llllll}0 & -6 & 5 & 76 & -1 & -17\end{array}$

$\begin{array}{rrrrrr}1 & -6 & 5 & 131 & 98 & 8 \\ 2 & -6 & 5 & 41 & 1 & -22\end{array}$ 
$\mathrm{h} \mathrm{k}$ l. LOFO $20 \mathrm{FC}$ lOs

$\begin{array}{rrrrrr}3 & -6 & 5 & 39 & 9 & -24 \\ 4 & -5 & 5 & 110 & -67 & 11 \\ 5 & -6 & 5 & 65 & -3 & -24 \\ 6 & -6 & 5 & 143 & -147 & 12 \\ 7 & -6 & 5 & 66 & 88 & -22 \\ 8 & -6 & 5 & 13 & -71 & -53 \\ 9 & -6 & 5 & 68 & 48 & -22 \\ 10 & -6 & 5 & 70 & -21 & -31 \\ -12 & -5 & 5 & 50 & -3 & -38 \\ -11 & -5 & 5 & 48 & 8 & -36 \\ -10 & -5 & 5 & 44 & 25 & -32 \\ -9 & -5 & 5 & 96 & -93 & 15 \\ -8 & -5 & 5 & 119 & 121 & 13 \\ -7 & -5 & 5 & 171 & -157 & 8 \\ -5 & -5 & 5 & 177 & 169 & 8 \\ -5 & -5 & 5 & 141 & -137 & 8 \\ -4 & -5 & 5 & 158 & 173 & 7 \\ -3 & -5 & 5 & 226 & -215 & 5 \\ -2 & -5 & 5 & 158 & 172 & 6 \\ -1 & -5 & 5 & 322 & -310 & 5 \\ 0 & -5 & 5 & 618 & 586 & 6 \\ 1 & -5 & 5 & 546 & -506 & 5 \\ 2 & -5 & 5 & 487 & 453 & 7 \\ 3 & -5 & 5 & 309 & -325 & 6 \\ 4 & -5 & 5 & 356 & 365 & 6 \\ 5 & -5 & 5 & 213 & -198 & 7 \\ 6 & -5 & 5 & 244 & 235 & 8 \\ 7 & -5 & 5 & 185 & -201 & 10 \\ 8 & -5 & 5 & 41 & 54 & -40 \\ 9 & -5 & 5 & 61 & -120 & -32 \\ 10 & -5 & 5 & 53 & -37 & -36\end{array}$

$\begin{array}{rrrrrr}9 & -5 & 5 & 61 & -120 & -32 \\ 10 & -5 & 5 & 53 & 37 & -36\end{array}$ $\begin{array}{rrrrrr}-12 & -4 & 5 & 65 & 13 & -31 \\ -12 & -4 & 5 & 65 & -10 & -21\end{array}$

$\begin{array}{rrrrrr}-11 & -4 & 5 & 65 & -10 & -21 \\ -10 & -4 & 5 & 50 & 37 & -25\end{array}$

$\begin{array}{llllll}-9 & -4 & 5 & 27 & 15 & -40 \\ -8 & -4 & 5 & 44 & -6 & -31\end{array}$

$\begin{array}{rrrrrr}-3 & -4 & 5 & 44 & -6 & -31 \\ -7 & -4 & 5 & 74 & -53 & -15\end{array}$

$\begin{array}{rrrrrr}-6 & -4 & 5 & 167 & 173 & 8 \\ -5 & -4 & 5 & 191 & -198 & 7\end{array}$

$\begin{array}{llllll}-4 & -4 & 5 & 150 & 128 & 7 \\ -3 & -4 & 5 & 107 & -98 & 8\end{array}$

$\begin{array}{rrrrrr}-3 & -4 & 5 & 107 & -98 & 8 \\ -2 & -4 & 5 & 65 & -58 & 11\end{array}$

$\begin{array}{rrrrrr}-1 & -4 & 5 & 376 & -380 & 6 \\ 0 & -4 & 5 & 228 & 233 & 5\end{array}$

$\begin{array}{lllll}1 & -4 & 5 & 230 & -232\end{array}$

$\begin{array}{rrrrrr}2 & -4 & 5 & 230 & -226 & 5 \\ 3 & -4 & 5 & 129 & 122 & 8\end{array}$

$\begin{array}{rrrrrr}3 & -4 & 5 & 129 & 122 & 8 \\ 4 & -4 & 5 & 87 & -36 & 11\end{array}$

$\begin{array}{rrrrrr}5 & -4 & 5 & 113 & -54 & 21 \\ 6 & -4 & 5 & 169 & 153 & 8\end{array}$

$\begin{array}{llllll}7 & -4 & 5 & 152 & 1.28 & 10\end{array}$

$\begin{array}{rrrrrr}8 & -4 & 5 & 92 & -96 & 17 \\ 9 & -4 & 5 & 66 & -10 & -21\end{array}$

$\begin{array}{rrrrrr}10 & -4 & 5 & 70 & 7 & -30 \\ 11 & -4 & 5 & 27 & -30 & -48\end{array}$

$\begin{array}{rrrrrr}11 & -4 & 5 & 27 & -30 & -48 \\ -12 & -3 & 5 & 76 & 24 & -21\end{array}$

$\begin{array}{llllll}-11 & -3 & 5 & 25 & 10 & -46 \\ -10 & -3 & 5 & 57 & -42 & -22\end{array}$

$\begin{array}{llllll}-9 & -3 & 5 & 60 & 37 & -26 \\ -8 & -3 & 5 & 44 & 52 & -28\end{array}$

$\begin{array}{llllll}-7 & -3 & 5 & 73 & -59 & -16\end{array}$

$\begin{array}{llllll}-6 & -3 & 5 & 214 & 221\end{array}$

$\begin{array}{rrrrr}-5 & -3 & 5 & 263 & -268 \\ -4 & -3 & 5 & 364 & 407\end{array}$

$\begin{array}{lllll}-3 & -3 & 5 & 417 & -423 \\ -2 & -3 & 5 & 353 & 396\end{array}$

$\begin{array}{lllll}-1 & -3 & 5 & 334 & -341\end{array}$

$\begin{array}{llllll}0 & -3 & 5 & 772 & 756\end{array}$

$\begin{array}{lllll}1 & -3 & 5 & 955 & -920\end{array}$

$\begin{array}{rrrrr}2 & -3 & 5 & 684 & 636 \\ 3 & -3 & 5 & 669 & -652\end{array}$

$\begin{array}{lllll}4 & -3 & 5 & 332 & 343\end{array}$

$\begin{array}{llllll}5 & -3 & 5 & 396 & -383 & 5\end{array}$

$\begin{array}{rrrrrr}6 & -3 & 5 & 276 & 274 & 6 \\ 7 & -3 & 5 & 201 & -197 & 8\end{array}$

$\begin{array}{rrrrrr}7 & -3 & 5 & 201 & -197 & 8 \\ 8 & -3 & 5 & 176 & 215 & 10\end{array}$

$\begin{array}{llllll}9 & -3 & 5 & 158 & -176 & 11\end{array}$

$\begin{array}{llllll}10 & -3 & 5 & 91 & 96 & 18\end{array}$

$\begin{array}{llllll}21 & -3 & 5 & 13 & -65 & -52\end{array}$

$\begin{array}{llllll}-12 & -2 & 5 & 57 & 20 & -33 \\ -11 & -2 & 5 & 25 & -3 & -46\end{array}$

$\begin{array}{llllll}-11 & -2 & 5 & 25 & -3 & -46 \\ -10 & -2 & 5 & 33 & -3 & -39\end{array}$

$\begin{array}{llllll}-9 & -2 & 5 & 47 & -29 & -30\end{array}$

$\begin{array}{rrrrrr}-8 & -2 & 5 & 100 & -104 & 14 \\ -7 & -2 & 5 & 115 & 114 & 11\end{array}$

$\begin{array}{rrrrrr}-6 & -2 & 5 & 50 & 34 & -25 \\ -5 & -2 & 5 & 67 & -86 & 33\end{array}$

$\begin{array}{llllll}-4 & -2 & 5 & 152 & 125 & 6\end{array}$

$\begin{array}{rrrrrr}-3 & -2 & 5 & 108 & 75 & 8 \\ -2 & -2 & 5 & 170 & -190 & 5\end{array}$ h $k 2$ 1OFO 1OFC 3.05

h 3 I 1OFO IOFC 10S

h $\mathrm{k} 1$ 1OFO IOFC 10s

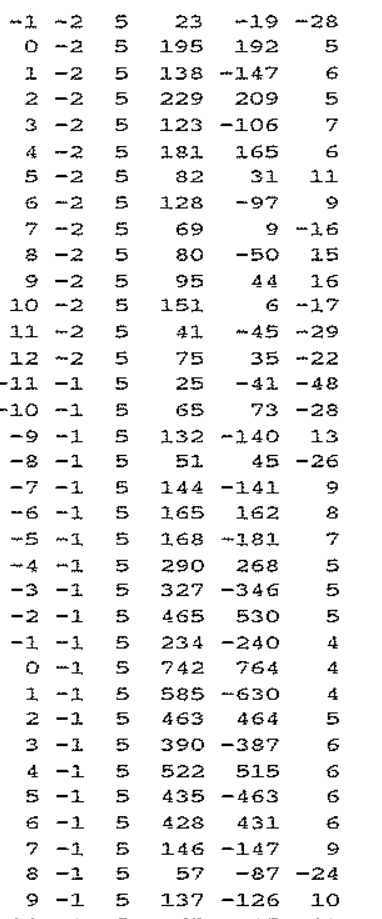

$\begin{array}{rrrrrr}-9 & 2 & 5 & 94 & -22 & 15 \\ -8 & 2 & 5 & 76 & -79 & -39 \\ -7 & 2 & 5 & 172 & 178 & 10 \\ -6 & 2 & 5 & 41 & 79 & -30 \\ -5 & 2 & 5 & 68 & 50 & 13 \\ -4 & 2 & 5 & 99 & -36 & 10 \\ -3 & 2 & 5 & 331 & -325 & 5 \\ -2 & 2 & 5 & 221 & 214 & 5 \\ -1 & 2 & 5 & 69 & 60 & 11 \\ 0 & 2 & 5 & 50 & -9 & -18 \\ 1 & 2 & 5 & 212 & 235 & 5 \\ 2 & 2 & 5 & 398 & -406 & 5 \\ 3 & 2 & 5 & 369 & -378 & 5 \\ 4 & 2 & 5 & 298 & -302 & 5 \\ 5 & 2 & 5 & 204 & -183 & 5 \\ 6 & 2 & 5 & 56 & -50 & -22 \\ 7 & 2 & 5 & 118 & 58 & 10 \\ 8 & 2 & 5 & 98 & -91 & 11 \\ 9 & 2 & 5 & 156 & -170 & 8 \\ 10 & 2 & 5 & 78 & -16 & -17\end{array}$

$\begin{array}{llllll}10 & 5 & 5 & 118 & 118 & 14\end{array}$

$\begin{array}{llllll}11 & 5 & 5 & 105 & -56 & 15 \\ 12 & 5 & 5 & 116 & 108 & 15\end{array}$

$\begin{array}{rrrrrr}12 & 5 & 5 & 116 & 108 & 15 \\ 13 & 5 & 5 & 67 & -44 & -25\end{array}$

$\begin{array}{rrrrrr}13 & 5 & 5 & 67 & -44 & -25 \\ -9 & 6 & 5 & 13 & 21 & -52\end{array}$

$\begin{array}{rrrrrr}-8 & 6 & 5 & 40 & -36 & -36 \\ -7 & 6 & 5 & 102 & 78 & 14\end{array}$

$\begin{array}{llllll}-6 & 6 & 5 & 55 & -45 & -29\end{array}$

$\begin{array}{llllll}-5 & 6 & 5 & 187 & 204 & 9\end{array}$

$\begin{array}{lllllll}-4 & 6 & 5 & 205 & \ldots 90 & 21\end{array}$

$\begin{array}{rrrrrr}-3 & 6 & 5 & 207 & -201 & 7 \\ -2 & 6 & 5 & 28 & 11 & -35\end{array}$

$\begin{array}{rrrrrr}-2 & 6 & 5 & 28 & 11 & -35 \\ -2 & 6 & 5 & 109 & 122 & 10\end{array}$

$\begin{array}{llllll}0 & 6 & 5 & 264 & 234 & 5\end{array}$

$\begin{array}{rrr}29 & -24 & -27 \\ 81 & -32 & 30\end{array}$

$\begin{array}{rr}81 & -32 \\ 361 & -357 \\ 233 & 231\end{array}$

$\begin{array}{rrrrrr}10 & 2 & 5 & 78 & -16 & -17 \\ 11 & 2 & 5 & 62 & 40 & -26\end{array}$

$\begin{array}{llllll}12 & 2 & 5 & 78 & 16 & -20\end{array}$

$\begin{array}{rrrrrr}13 & 2 & 5 & 41 & 5 & -33 \\ -10 & 3 & 5 & 75 & 25 & -27\end{array}$

$\begin{array}{llllll}-9 & 3 & 5 & 97 & -86 & 16\end{array}$

$\begin{array}{llllll}-8 & 3 & 5 & 145 & 152 & 12\end{array}$

$\begin{array}{rrrrrr}-7 & 3 & 5 & 172 & -183 & 10 \\ -6 & 3 & 5 & 289 & 296 & 6\end{array}$

$\begin{array}{llllll}-5 & 3 & 5 & 80 & -82 & 14\end{array}$

$\begin{array}{llllll}-4 & 3 & 5 & 270 & 276 & 6\end{array}$

$\begin{array}{rrrrr}-3 & 3 & 5 & 566 & -602 \\ -2 & 3 & 5 & 473 & 480\end{array}$

$\begin{array}{rrrrr}-1 & 3 & 5 & 337 & -329 \\ 0 & 3 & 5 & 953 & 892\end{array}$

$\begin{array}{llllll}1 & 3 & 5 & 438 & -428\end{array}$

$\begin{array}{rrrrrr}9 & -1 & 5 & 137 & -126 & 10 \\ 10 & -1 & 5 & 67 & 95 & -21\end{array}$

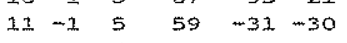

$\begin{array}{rrrrrr}12 & -1 & 5 & 64 & 52 & -27 \\ -11 & 0 & 5 & 51 & -4 & -23\end{array}$

$\begin{array}{llllll}-10 & 0 & 5 & 48 & -37 & -28\end{array}$

$\begin{array}{rrrrrr}-9 & 0 & 5 & 106 & 57 & 10 \\ -8 & 0 & 5 & 55 & -17 & -22\end{array}$

$\begin{array}{rrrrrr}-8 & 0 & 5 & 55 & -17 & -22 \\ -7 & 0 & 5 & 100 & -93 & 10\end{array}$

$\begin{array}{rrrrrr}-7 & 0 & 5 & 100 & -93 & 10 \\ -6 & 0 & 5 & 125 & 113 & 7\end{array}$

$\begin{array}{llllll}-5 & 0 & 5 & 71 & -30 & -15\end{array}$

$\begin{array}{rrrrrr}-4 & 0 & 5 & 92 & 64 & 6 \\ -3 & 0 & 5 & 110 & -119 & 6\end{array}$

$\begin{array}{rrrrrr}-2 & 0 & 5 & 18 & 44 & -22 \\ -1 & 0 & 5 & 284 & -281 & 4\end{array}$

$\begin{array}{rrrrrr}-1 & 0 & 5 & 284 & -281 & 4 \\ 0 & 0 & 5 & 239 & 143 & -6\end{array}$

$\begin{array}{rrrrrr}1 & 0 & 5 & 203 & 221 & 3 \\ 2 & 0 & 5 & 309 & -317 & 5\end{array}$

$\begin{array}{rrrrrr}2 & 0 & 5 & 309 & -317 & 5 \\ 3 & 0 & 5 & 288 & 291 & 4\end{array}$

$\begin{array}{rllllr}4 & 0 & 5 & 207 & 149 & 7 \\ 5 & 0 & 5 & 123 & -43 & -5\end{array}$

$\begin{array}{llllll}6 & 0 & 5 & 73 & -65 & 10\end{array}$

$\begin{array}{rrrrrr}7 & 0 & 5 & 115 & 95 & 13 \\ 8 & 0 & 5 & 56 & 31 & -14\end{array}$

$\begin{array}{rrrrrr}9 & 0 & 5 & 85 & -68 & 11 \\ 10 & 0 & 5 & 109 & -133 & 11\end{array}$

$11005655-23$

$\begin{array}{llllll}12 & 0 & 5 & 64 & -7 & -21\end{array}$

$\begin{array}{rrrrrr}13 & 0 & 5 & 51 & 1 & -21 \\ -11 & 1 & 5 & 65 & -45 & -29\end{array}$

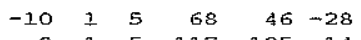

$\begin{array}{llllll}-9 & 1 & 5 & 117 & -105 & 14\end{array}$

$\begin{array}{rrrrrr}-8 & 1 & 5 & 102 & 133 & 16 \\ -7 & 1 & 5 & 56 & -41 & -21\end{array}$

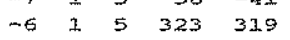

$\begin{array}{rrrrr}-5 & 1 & 5 & 452 & -438 \\ -4 & 1 & 5 & 483 & 497\end{array}$

$\begin{array}{lllll}-3 & 1 & 5 & 178 & -178\end{array}$

$\begin{array}{llllll}-2 & 1 & 5 & 213 & 204 & 5\end{array}$

$\begin{array}{llllll}-1 & 1 & 5 & 34 & -123 & -23\end{array}$

$\begin{array}{rrrcr}1 & 1 & 5 & 868 & 921 \\ 1 & 5 & 1419 & -1500\end{array}$

$\begin{array}{lllll}2 & 1 & 5 & 475 & 487\end{array}$

$\begin{array}{lllll}3 & 1 & 5 & 750 & -807\end{array}$

$\begin{array}{rrrrrr}4 & 1 & 5 & 308 & 287 & 5 \\ 5 & 1 & 5 & 106 & -114 & 9\end{array}$

$\begin{array}{rrrrrr}6 & 1 & 5 & 337 & 342 & 5 \\ 7 & 1 & 5 & 216 & -189 & 7\end{array}$

$815 \quad 80-15 \quad 14$

$\begin{array}{rrrrrr}9 & 1 & 5 & 78 & 10 & 15 \\ 10 & 1 & 5 & 115 & 84 & 12\end{array}$

$\begin{array}{rrrrrr}11 & 1 & 5 & 53 & -30 & -32 \\ 12 & 1 & 5 & 25 & 17 & -48\end{array}$

$\begin{array}{llllll}13 & 1 & 5 & 13 & 9 & -53\end{array}$

$\begin{array}{rrrrrr}-11 & 2 & 5 & 60 & 1 & -33 \\ -10 & 2 & 5 & 13 & 35 & -53\end{array}$ h $\mathrm{k}$ I 10FO 1OFC $10 \mathrm{~s}$

$-510 \quad 5-525070-35$ $\begin{array}{rrrrrr}-4 & 10 & 5 & 52 & 79 & -35 \\ -4 & 10 & 51 & -30 & -26\end{array}$ $\begin{array}{llllll}-3 & 10 & 5 & 56 & 28 & -30\end{array}$

$\begin{array}{rrrrrr}-2 & 10 & 5 & 84 & 42 & 15\end{array}$

$\begin{array}{llllll}-1 & 10 & 5 & 53 & -45 & -25\end{array}$

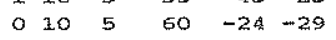

$\begin{array}{rrrrrr}1 & 10 & 5 & 118 & -103 & 12 \\ 2 & 30 & 5 & 97 & 102 & 14\end{array}$

$\begin{array}{llllll}3 & 10 & 5 & 156 & -139 & 9\end{array}$

$\begin{array}{llllll}4 & 10 & 5 & 90 & -13 & 15\end{array}$

$510557 \quad-12-20$

$\begin{array}{rrrrrr}6 & 20 & 5 & 61 & 3 & -22 \\ 7 & 10 & 5 & 92 & -18 & 17\end{array}$

$\begin{array}{llllll}8 & 10 & 5 & 81 & 27 & -18\end{array}$

$\begin{array}{llllll}9 & 10 & 5 & 91 & -31 & 18\end{array}$

$2010557 \quad 3-34$

$\begin{array}{llllll}12 & 10 & 5 & 40 & 41 & -41 \\ 12 & 10 & 5 & 46 & 35 & -41\end{array}$

$\begin{array}{llllll}-4 & 11 & 5 & 149 & 152 & 13\end{array}$

$\begin{array}{llllll}-3 & 11 & 5 & 137 & -151 & 14\end{array}$

$\begin{array}{llllll}-2 & 11 & 5 & 151 & 175 & 12\end{array}$

$\begin{array}{rrrrrr}-1 & 11 & 5 & 187 & -184 & 10 \\ 0 & 11 & 5 & 265 & 265 & 8\end{array}$

$\begin{array}{llllll}1 & 11 & 5 & 307 & -306 \quad 8\end{array}$

$\begin{array}{llllll}2 & 11 & 5 & 386 & 204 & 10\end{array}$

$\begin{array}{llllll}3 & 11 & 5 & 186 & -180 & 8\end{array}$

$\begin{array}{rrrrrr}4 & 11 & 5 & 213 & 220 & 8 \\ 5 & 11 & 5 & 201 & -196 & 8\end{array}$

$\begin{array}{llllll}6 & 11 & 5 & 133 & 129 & 23\end{array}$

$7115 \quad 110-116 \quad 15$

$\begin{array}{llllll}9 & 11 & 5 & 12 & -80 & -50\end{array}$

$1011531 \quad 63-48$

$\begin{array}{rrrrrr}11 & 11 & 5 & 26 & -54 & -53 \\ 12 & 11 & 5 & 93 & 26 & -20\end{array}$

$\begin{array}{llllll}-3 & 12 & 5 & 63 & -3 & -32\end{array}$

$\begin{array}{llllll}-2 & 12 & 5 & 26 & 30 & -50\end{array}$

$\begin{array}{llllll}-1 & 12 & 5 & 56 & -54 & -33\end{array}$

$\begin{array}{llllll}0 & 12 & 5 & 45 & 20 & -34 \\ 1 & 12 & 5 & 31 & 17 & -35\end{array}$

$\begin{array}{llllll}2 & 12 & 5 & 37 & 25 & -31\end{array}$

$\begin{array}{llllll}3 & 12 & 5 & 76 & -80 & -16\end{array}$

$\begin{array}{lllll}4 & 12 & 109 & 94 & 12\end{array}$

$\begin{array}{rrrrrr}5 & 12 & 5 & 48 & 62 & -34 \\ 7 & 12 & 5 & 105 & -42 & 14\end{array}$

$\begin{array}{llllll}8 & 12 & 5 & 49 & -3 & -34\end{array}$

$912569 \quad 0-22$

$\begin{array}{llllll}10 & 12 & 5 & 37 & -6 & -46\end{array}$

$\begin{array}{rrrrrr}11 & 12 & 5 & 72 & -23 & -32 \\ -1 & 13 & 5 & 122 & -132 & 16\end{array}$

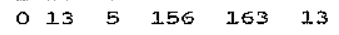

$\begin{array}{llllll}1 & 13 & 5 & 213 & -213 & 10\end{array}$

$\begin{array}{llllll}2 & 13 & 5 & 140 & 155 & 14\end{array}$

$\begin{array}{llllll}3 & 13 & 5 & 98 & -133 & 17\end{array}$

$\begin{array}{rrrrrr}4 & 13 & 5 & 158 & 152 & 10 \\ 5 & 13 & 5 & 105 & -111 & 13\end{array}$

$\begin{array}{llllll}6 & 13 & 5 & 63 & 118 & -31\end{array}$

$\begin{array}{llllll}7 & 13 & 5 & 74 & -44 & -22\end{array}$

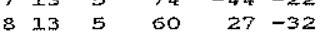

$\begin{array}{rrrrrr}9 & 13 & 5 & 50 & -29 & -35 \\ 10 & 13 & 5 & 93 & 26 & -20\end{array}$

$\begin{array}{llllll}1 & 14 & 5 & 33 & -73 & -45\end{array}$

$\begin{array}{llllll}214 & 5 & 61 & 65 & -32\end{array}$

$\begin{array}{llllll}3 & 14 & 5 & 90 & -98 & -20\end{array}$

$414 \quad 5 \quad 29 \quad 65-45$

$\begin{array}{rrrrrr}5 & 14 & 5 & 107 & -77 & 14 \\ 6 & 14 & 5 & 98 & 86 & 18\end{array}$

$\begin{array}{llllll}7 & 14 & 5 & 55 & -74 & -38\end{array}$

$8145 \quad 42 \quad 52-43$

$\begin{array}{lllll}-5-13 & 6 & 63 & -51 & -29\end{array}$

$\begin{array}{rrrrr}-4-13 & 6 & 71 & 63 & -22 \\ -3-13 & 6 & 88 & -94 & -18\end{array}$

$\begin{array}{lllll}-2-13 & 6 & 106 & 91 & 17\end{array}$

$\begin{array}{lllll}-1-13 & 6 & 56 & -47 & -32\end{array}$

$-7-126 \quad 115-106 \quad 15$

$\begin{array}{rrrrr}-6-12 & 6 & 42 & 99 & -40 \\ -5-12 & 6 & 50 & -70 & -33\end{array}$

$-4-126210 \quad 110 \quad 15$

$\begin{array}{lllll}-3-12 & 6 & 92 & -1.15 & 18\end{array}$

$\begin{array}{lllll}-2-12 & 6 & 98 & 102 & 17\end{array}$

$\begin{array}{lllll}-1-12 & 6 & 53 & -118 & -36\end{array}$

$\begin{array}{lllll}0-12 & 6 & 174 & 280 & 11\end{array}$

$1-12 \quad 6 \quad 135-165 \quad 16$

$\begin{array}{rrrrr}2-12 & 6 & 133 & 159 & 17 \\ -8-11 & 6 & 52 & 20 & -28\end{array}$

$\begin{array}{lllrr}-8-11 & 6 & 52 & 20 & -28 \\ -7-11 & 6 & 93 & -15 & -24\end{array}$

$\begin{array}{rrrrr}-6-11 & 6 & 58 & 40 & -32 \\ -5-11 & 6 & 107 & -108 & 14\end{array}$

$\begin{array}{lllll}-4-11 & 6 & 141 & 126 & 10\end{array}$

$\begin{array}{lllll}-3-11 & 6 & 41 & -87 & -34\end{array}$ 


\section{h $k$ I IOFO IOFC lOs h $\mathrm{k}$ I IOFO IOFC $10 \mathrm{~s}$}

$\begin{array}{rrrrr}-1-11 & 6 & 46 & -48 & -33 \\ 0-11 & 6 & 47 & 82 & -28 \\ 1-11 & 6 & 61 & -50 & -24 \\ 2-11 . & 6 & 83 & 68 & -21 \\ 3-11 & 6 & 49 & -54 & -38 \\ 4-11 & 6 & 198 & 21 & -18 \\ -9-10 & 6 & 26 & -22 & -48 \\ -8-10 & 6 & 39 & -13 & -39\end{array}$

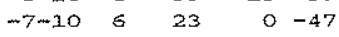

$\begin{array}{rrrrr}-6-10 & 6 & 59 & 79 & -30 \\ -5-10 & 6 & 118 & -135 & 33\end{array}$

$\begin{array}{lllll}-4-10 & 6 & 133 & 153 & 12\end{array}$

$\begin{array}{lllll}-3-10 & 6 & 208 & -212 & 8 \\ -2-10 & 5 & 249 & 239 & 8\end{array}$

$\begin{array}{rrrrr}-2-10 & 6 & 249 & 239 & 8 \\ -1-10 & 6 & 213 & -222 & 9\end{array}$

$\begin{array}{lllll}0-10 & 6 & 172 & 186 & 1.1\end{array}$

$1-10 \quad 6 \quad 164-166 \quad 11$

$\begin{array}{rrrrr}2-10 & 6 & 194 & 187 & 11 \\ 3-10 & 6 & 170 & -169 & 11\end{array}$

$\begin{array}{rrrrr}3-10 & 6 & 170 & -169 & 11 \\ 4-10 & 6 & 149 & 164 & 14\end{array}$

$\begin{array}{llllll}5-10 & 6 & 140 & -144 & 14\end{array}$

$\begin{array}{rrrrrr}-10 & -9 & 6 & 32 & 13 & -45 \\ -9 & -9 & 6 & 31 & -37 & -46\end{array}$

$\begin{array}{llllll}-8 & -9 & 6 & 53 & 27 & -28\end{array}$

$\begin{array}{rrrrrr}-7 & -9 & 6 & 73 & -22 & -19 \\ -6 & -9 & 6 & 73 & 33 & -17\end{array}$

$\begin{array}{llllll}-5 & -9 & 6 & 83 & 51 & 15\end{array}$

$\begin{array}{llllll}-3 & -9 & 6 & 115 & -138 & 12\end{array}$

$\begin{array}{llllll}-2 & -9 & 6 & 91 & 109 & 15\end{array}$

$\begin{array}{rrrrrr}-1 & -9 & 6 & 85 & -84 & 16 \\ 0 & -9 & 6 & 109 & 126 & 13\end{array}$

$\begin{array}{llllll}1 & -9 & 6 & 96 & -91 & 25\end{array}$

$\begin{array}{rrrrrr}2 & -9 & 6 & 91 & 88 & 17 \\ 3 & -9 & 6 & 54 & -80 & -32\end{array}$

$\begin{array}{rrrrrr}3 & -9 & 6 & 54 & -80 & -32 \\ 4 & -9 & 6 & 23 & -23 & -42 \\ 5 & -9 & 6 & 49 & 14 & -31\end{array}$

$\begin{array}{llllll}5 & -9 & 6 & 49 & 14 & -31 \\ 6 & -9 & 6 & 75 & 10 & -23\end{array}$

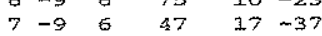

$\begin{array}{llllll}-10 & -8 & 6 & 101 & 75 & 16\end{array}$

$\begin{array}{rrrrrr}-9 & -8 & 6 & 102 & -83 & 15 \\ -8 & -8 & 6 & 83 & 47 & 16\end{array}$

$\begin{array}{llllll}-7 & -8 & 6 & 79 & -88 & -18\end{array}$

$\begin{array}{llllll}-6 & -8 & 6 & 128 & 137 & 12\end{array}$

$\begin{array}{rrrrrr}-5 & -8 & 6 & 171 & -147 & 9 \\ -4 & -8 & 6 & 208 & 205 & 8\end{array}$

$\begin{array}{llllll}-3 & -8 & 6 & 54 & -8 & -17\end{array}$

$\begin{array}{llllll}-2 & -8 & 6 & 48 & 22 & -23\end{array}$

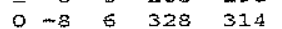

$\begin{array}{llllll}1 & -8 & 6 & 320 & -337 & 7\end{array}$

$\begin{array}{rrrrrr}2 & -8 & 6 & 306 & 325 & 7 \\ 3 & -8 & 6 & 233 & -249 & 8\end{array}$

$\begin{array}{llllll}4 & -8 & 6 & 182 & 195 & 11\end{array}$

$5-8$ \& $254-2629$

$5-8 \quad 6 \quad 134 \quad 150 \quad 13$

$\begin{array}{rrrrrr}7 & -8 & 6 & 92 & -120 & -21 \\ 8 & -8 & 6 & 43 & 64 & -40\end{array}$

$\begin{array}{llllll}-11 & -7 & 6 & 92 & -8 & -25\end{array}$

$\begin{array}{rrrrrr}-9 & -7 & 6 & 73 & -20 & -19\end{array}$

$\begin{array}{llllll}-8 & -7 & 6 & 53 & 6 & -30\end{array}$

$\begin{array}{rrrrrr}-7 & -7 & 6 & 42 & 10 & -31 \\ -6 & -7 & 6 & 86 & 23 & 15\end{array}$

$\begin{array}{llllll}-5 & -7 & 6 & 90 & -121 & 12\end{array}$

$\begin{array}{lllllll}-4 & -7 & 6 & 243 & 258 & 6\end{array}$

$\begin{array}{rrrrrr}-3 & -7 & 6 & 193 & -196 & 7 \\ -2 & -7 & 6 & 210 & 221 & 6\end{array}$

$\begin{array}{rrrrrr}-2 & -7 & 6 & 210 & 221 & 6 \\ -1 & -7 & 6 & 36 & 20 & -32\end{array}$

$\begin{array}{lllll}0 & -7 & 6 & 120 & -85\end{array}$

$\begin{array}{llllll}1 & -7 & 6 & 104 & 99 & 12\end{array}$

$\begin{array}{llllll}2 & -7 & 6 & 54 & -4 & -27\end{array}$

$\begin{array}{rrrrrr}3 & -7 & 6 & 25 & 8 & -38 \\ 4 & -7 & 6 & 61 & -46 & -20\end{array}$

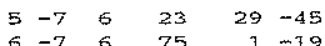

$\begin{array}{llllll}7 & -7 & 6 & 49 & -18 & -33\end{array}$

$\begin{array}{llllll}8 & -7 & 6 & 64 & -37 & -3 I\end{array}$

$9-7 \quad 6 \quad 54 \quad 9-33$

$\begin{array}{llllll}-11 & -6 & 6 & 45 & -8 & -32 \\ -10 & -6 & 6 & 78 & 45 & -19\end{array}$

$\begin{array}{llllll}-9 & -6 & 6 & 71 & -74 & -27\end{array}$

$-8-66106$ 70 14

$\begin{array}{rrrrrr}-7 & -6 & 6 & 85 & -53 & 15 \\ -6 & -6 & 6 & 74 & 93 & -15\end{array}$

$\begin{array}{llllll}-5 & -6 & 6 & 124 & -124 & 10\end{array}$

$\begin{array}{rrrrrr}-4 & -6 & 6 & 74 & 73 & 13 \\ -3 & -6 & 6 & 185 & -194 & 7\end{array}$

$\begin{array}{rrrrrr}-2 & -6 & 6 & 260 & 250 & 5 \\ -1 & -6 & 6 & 277 & -260 & 5\end{array}$ $\begin{array}{lllll}0 & -6 & 241 & 259\end{array}$

$\begin{array}{rrrrr}2 & -6 & 6 & 331 & 307\end{array}$

$\begin{array}{llllll}3 & -6 & 6 & 297 & -296\end{array}$

$\begin{array}{lllll}4 & -6 & 6 & 250 & 252\end{array}$

$\begin{array}{rrrrrr}5 & -6 & 6 & 171 & -185 & 10 \\ 6 & -6 & 6 & 127 & 152 & 13\end{array}$

$\begin{array}{lllll}7 & 6 & 115 & -129 & 15\end{array}$

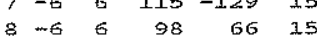

$\begin{array}{rrrrrr}-11 & -5 & 6 & 49 & -29 & -29 \\ -10 & -5 & 6 & 45 & 46 & -36\end{array}$

$\begin{array}{llllll}-9 & -5 & 6 & 28 & -53 & -44\end{array}$

$\begin{array}{rrrrrr}-8 & -5 & 6 & 65 & 21 & -20 \\ -7 & -5 & 6 & 38 & -35 & -32\end{array}$

$\begin{array}{rrrrrr}-7 & -5 & 6 & 38 & -35 & -32 \\ -6 & -5 & 6 & 155 & 136 & 8\end{array}$

$\begin{array}{llllll}-5 & -5 & 6 & 48 & -36 & -19\end{array}$

$\begin{array}{rrrrrr}-4 & -5 & 6 & 99 & 72 & 10 \\ -3 & -5 & 6 & 63 & -82 & -13\end{array}$

$\begin{array}{rrrrrr}-3 & -5 & 6 & 63 & -82 & -13 \\ -2 & -5 & 6 & 66 & -26 & 12\end{array}$

$\begin{array}{rrrrrr}-1 & -5 & 6 & 81 & 86 & 11\end{array}$

$\begin{array}{lllll}0 & -5 & 6 & 124 & 143\end{array}$

$\begin{array}{rrrrrr}2 & -5 & 6 & 24 d & -213 & 5 \\ 3 & -5 & 6 & 48 & 57 & -19\end{array}$

$\begin{array}{rrrrrr}4 & -5 & 6 & 233 & -239 & 7 \\ 5 & -5 & 6 & 123 & 130 & 11\end{array}$

$\begin{array}{rrrrrr}5 & -5 & 6 & 123 & 130 & 11 \\ 6 & -5 & 6 & 102 & 59 & 1.4\end{array}$

$\begin{array}{llllll}7 & -5 & 6 & 92 & 78 & 15\end{array}$

$\begin{array}{llllll}8 & -5 & 6 & 88 & -97 & 16\end{array}$

$\begin{array}{rrrrrr}9 & -5 & 6 & 31 & 38 & -44 \\ 10 & -5 & 6 & 46 & -38 & -35\end{array}$

$\begin{array}{llllll}-11 & -4 & 6 & 66 & -20 & -24\end{array}$

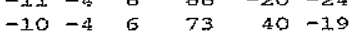

$\begin{array}{rrrrrr}-9 & -4 & 6 & 48 & -16 & -32 \\ -8 & -4 & 6 & 45 & 8 & -29\end{array}$

$\begin{array}{rrrrrr}-8 & -4 & 6 & 45 & 8 & -29 \\ -7 & -4 & 6 & 89 & -49 & 15\end{array}$

$\begin{array}{llllll}-6 & -4 & 6 & 142 & 131 & 8\end{array}$

$\begin{array}{lllll}-5 & -4 & 6 & 351 & -353\end{array}$

$\begin{array}{lllll}-7 & -4 & 6 & 473 & 487\end{array}$

$\begin{array}{rrrrr}-3 & -4 & 6 & 354 & -358 \\ -2 & -4 & 6 & 277 & 294\end{array}$

$\begin{array}{lllll}-1 & -4 & 6 & 296 & -312\end{array}$

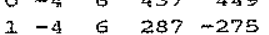

$2-4 \quad 6 \quad 439 \quad 413$

$\begin{array}{lllll}3 & -4 & 6 & 533 & -517\end{array}$

$\begin{array}{lllll}4 & -4 & 6 & 247 & 275\end{array}$

$5-4 \quad 6 \quad 359-360 \quad 6$

$\begin{array}{rrrrrr}6 & -4 & 6 & 149 & 136 & 10 \\ 7 & -4 & 6 & 75 & -90 & -17\end{array}$

$\begin{array}{rrrrrr}7 & -4 & 6 & 75 & -90 & -17 \\ 8 & -4 & 6 & 98 & 106 & 15\end{array}$

$\begin{array}{llllll}9 & -4 & 6 & 56 & -59 & -23\end{array}$

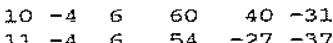

$\begin{array}{rrrrrr}11 & -4 & 6 & 54 & -27 & -37 \\ -11 & -3 & 6 & 51 & -3 & -32 \\ -110 & -3 & 6 & 65 & 22 & -28\end{array}$

$\begin{array}{rrrrrr}-10 & -3 & 6 & 65 & 22 & -28 \\ -9 & -3 & 6 & 92 & -79 & 16\end{array}$

$\begin{array}{llllll}-8 & -3 & 6 & 12 & 13 & -49\end{array}$

$\begin{array}{llllll}-7 & -3 & 6 & 119 & 87 & 11\end{array}$

$\begin{array}{rrrrrr}-6 & -3 & 6 & 58 & 21 & -17 \\ -5 & -3 & 6 & 47 & -13 & -24\end{array}$

$\begin{array}{rrrrrr}-5 & -3 & 6 & 47 & -13 & -24 \\ -4 & -3 & 6 & 85 & 10 & 10\end{array}$

$\begin{array}{llllll}-3 & -3 & 6 & 198 & -215 & 6 \\ -2 & -3 & 6 & 334 & 341 & 5\end{array}$

$\begin{array}{rrrrrr}-2 & -3 & 6 & 334 & 341 & 5 \\ -1 & -3 & 6 & 21 & 23 & -30\end{array}$

$\begin{array}{llllll}0 & -3 & 6 & 28 & 40 & -27\end{array}$

$\begin{array}{rrrrrr}1 & -3 & 6 & 158 & -150 & 6 \\ 2 & -3 & 6 & 103 & 78 & 9\end{array}$

$\begin{array}{rrrrrr}3 & -3 & 6 & 85 & -55 & 11\end{array}$

$\begin{array}{rrrrrr}4 & -3 & 6 & 112 & -53 & 9 \\ 5 & -3 & 6 & 66 & 46 & -25\end{array}$

$\begin{array}{llllll}5 & -3 & 6 & 66 & 46 & -15 \\ 6 & -3 & 6 & 47 & 71 & -29\end{array}$

$\begin{array}{rrrrrr}8 & -3 & 6 & 78 & -107 & -17 \\ 9 & -3 & 6 & 105 & 37 & 14\end{array}$

$\begin{array}{llllll}10 & -3 & 6 & 60 & -25 & -31\end{array}$

$\begin{array}{llllll}11 & -3 & 6 & 26 & 4 & -48\end{array}$

$\begin{array}{rrrrrr}-11 & -2 & 6 & 75 & -46 & -27 \\ -10 & -2 & 6 & 53 & 66 & -32\end{array}$

$\begin{array}{llllll}-9 & -2 & 6 & 85 & -84 & -17\end{array}$

$\begin{array}{rrrrrr}-8 & -2 & 6 & 42 & -28 & -30 \\ -7 & -2 & 6 & 104 & -104 & 12\end{array}$

$\begin{array}{rrrrrr}-7 & -2 & 6 & 104 & -104 & 12 \\ -6 & -2 & 6 & 190 & 193 & 7\end{array}$

$\begin{array}{lllll}-5 & -2 & 6 & 124 & -110\end{array}$

$\begin{array}{rrrrr}-4 & -2 & 6 & 264 & 256 \\ -3 & -2 & 6 & 323 & -340\end{array}$

$\begin{array}{llllll}-2 & -2 & 6 & 511 & 541\end{array}$

$\begin{array}{llllll}-1 & -2 & 6 & 467 & -486 & 5\end{array}$ $\begin{array}{llllll}9 & -6 & 6 & 57 & -69 & -27\end{array}$

$\begin{array}{llllll}1 & -5 & 6 & 126 & -115 & 8 \\ 2 & -5 & 6 & 244 & -213 & 5\end{array}$

$\begin{array}{llllll}7 & -3 & 6 & 128 & -124 & 11\end{array}$ $\begin{array}{lllll}1 & -2 & 6 & 660 & -662\end{array}$

$\begin{array}{rrrrr}1 & -2 & 6 & 660 & -662 \\ 2 & -2 & 6 & 371 & 386\end{array}$

$\begin{array}{llllll}3 & -2 & 6 & 454 & -459\end{array}$

$\begin{array}{lllll}4 & -2 & 6 & 499 & 511\end{array}$

$\begin{array}{lllll}5 & -2 & 6 & 449 & -462\end{array}$

$\begin{array}{rrrrr}6 & -2 & 6 & 341 & 327 \\ 7 & -2 & 6 & 125 & -133\end{array}$

$\begin{array}{rrrrrr}8 & -2 & 6 & 12 & 53 & -48\end{array}$

$\begin{array}{lllllll}9 & -2 & 6 & 124 & -158 & 13\end{array}$

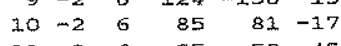

$\begin{array}{rrrrrr}11 & -2 & 6 & 25 & -50 & -45 \\ 12 & -2 & 6 & 60 & 23 & -29\end{array}$

$\begin{array}{llllll}-11 & -1 & 6 & 13 & -13 & -51\end{array}$

$\begin{array}{lllllll}-10 & -1 & 6 & 68 & -26 & -21\end{array}$

$\begin{array}{rrrrrr}-9 & -1 & 6 & 12 & 32 & -46 \\ -8 & -1 & 6 & 45 & -38 & -28\end{array}$

$\begin{array}{rrrrrr}-7 & -1 & 6 & 119 & 68 & 11 \\ -6 & -3 & 6 & 81 & -69 & 33\end{array}$

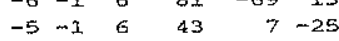

$\begin{array}{llllll}-4 & -1 & 6 & 75 & 36 & 11 \\ -3 & -1 & 6 & 72 & 54 & 10\end{array}$

$\begin{array}{rrrrrr}-3 & -1 & 6 & 72 & 54 & 10 \\ -2 & -1 & 6 & 118 & -119 & 7\end{array}$

$\begin{array}{llllll}-1 & -1 & 6 & 130 & 121 & 6 \\ 0 & -1 & 6 & 202 & 234 & 5\end{array}$

$\begin{array}{rrrrrr}0 & -1 & 6 & 202 & 234 & 5 \\ 1 & -1 & 6 & 328 & -340 & 5\end{array}$

$\begin{array}{rrrrrr}2 & -1 & 6 & 80 & -93 & 10 \\ 3 & -1 & 6 & 406 & -367 & 6\end{array}$

$\begin{array}{rrrrrr}3 & -1 & 6 & 406 & -367 & 6 \\ 4 & -1 & 6 & 120 & 60 & 8\end{array}$

$\begin{array}{llllll}5 & -1 & 6 & 53 & 14 & -23\end{array}$

$\begin{array}{rrrrrr}6 & -1 & 6 & 258 & 232 & 6 \\ 7 & -1 & 6 & 140 & -135 & 9\end{array}$

$\begin{array}{rrrrrr}7 & -1 & 6 & 140 & -135 & 9 \\ 8 & -1 & 6 & 10 & -4 & -41\end{array}$

$\begin{array}{llllll}9 & -1 & 6 & 73 & 33 & -18 \\ 0 & -1 & 6 & 51 & -1 & -31\end{array}$

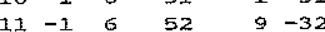

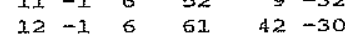

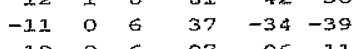

$\begin{array}{rrrrrr}-10 & 0 & 6 & 97 & 96 & 11 \\ -9 & 0 & 6 & 114 & -128 & 11\end{array}$

$\begin{array}{lllllll}-8 & 0 & 6 & 134 & 133 & 8\end{array}$

$\begin{array}{rrrrrr}-7 & 0 & 6 & 77 & -47 & -18 \\ -6 & 0 & 6 & 284 & 288 & 7\end{array}$

$\begin{array}{rrrrrr}-6 & 0 & 6 & 284 & 288 & 7 \\ -5 & 0 & 6 & 218 & -226 & 4\end{array}$

$\begin{array}{llllll}-4 & 0 & 6 & 160 & 174 & 11\end{array}$

$\begin{array}{rrrrrr}-3 & 0 & 6 & 231 & -236 & 3 \\ -2 & 0 & 6 & 404 & 426 & 4\end{array}$

$\begin{array}{rrrrr}-2 & 0 & 6 & 404 & 426 \\ -1 & 0 & 5 & 443 & -445\end{array}$

$\begin{array}{rlllrr}0 & 0 & 6 & 397 & 448 \\ 1 & 0 & 6 & 230 & -252\end{array}$

$\begin{array}{llllll}1 & 0 & 6 & 190 & 184 & 5\end{array}$

$\begin{array}{llllll}3 & 0 & 6 & 896 & -896 & 13\end{array}$

$\begin{array}{rrrrr}4 & 0 & 6 & 209 & 196\end{array}$

$\begin{array}{rrrrrr}5 & 0 & 6 & 704 & -727 & 4 \\ 6 & 0 & 6 & 339 & 367 & 4\end{array}$

$\begin{array}{rlllll}7 & 0 & 6 & 339 & 367 & 4 \\ 9 & 0 & 6 & 179 & -172 & 12\end{array}$

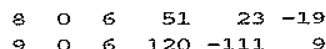

$\begin{array}{rrrrrr}9 & 0 & 6 & 120 & -111 & 9 \\ 10 & 0 & 6 & 91 & 44 & -22\end{array}$

$\begin{array}{rlllrl}11 & 0 & 6 & 78 & -11 & -18 \\ 12 & 0 & 6 & 85 & 20 & -23\end{array}$

$\begin{array}{llllll}13 & 0 & 6 & 63 & 16 & -23\end{array}$

$\begin{array}{llllll}-11 & 1 & 6 & 56 & -1 & -32 \\ -10 & 1 & 6 & 76 & 34 & -19\end{array}$

$\begin{array}{rrrrrr}-10 & 1 & 6 & 76 & 34 & -19 \\ -9 & 1 & 6 & 100 & -15 & 15\end{array}$

$\begin{array}{rrrrrr}-8 & 1 & 6 & 86 & -41 & 16 \\ -7 & 1 & 6 & 114 & 85 & 12\end{array}$

$\begin{array}{llllll}-6 & 1 & 6 & 196 & 196 & 7\end{array}$

$\begin{array}{rrrrrr}-5 & 1 & 6 & 41 & 31 & -29 \\ -4 & 1 & 6 & 87 & 49 & 11\end{array}$

$\begin{array}{rrrrrr}-4 & 1 & 6 & 87 & 49 & 11 \\ -3 & 1 & 6 & 194 & -187 & 5\end{array}$

$\begin{array}{llllll}-2 & 1 & 6 & 40 & 34 & -22\end{array}$

$\begin{array}{llllll}-1 & 1 & 6 & 64 & -27 & 11\end{array}$

$\begin{array}{rrrrrr}0 & 1 & 6 & 164 & 186 & 6 \\ 1 & 1 & 6 & 193 & -196 & 5\end{array}$

$\begin{array}{rrrrrr}1 & 1 & 6 & 193 & -196 & 5 \\ 3 & 1 & 6 & 252 & -287 & 4 \\ 4 & 6 & 42 & 62 & -17\end{array}$

$\begin{array}{rrrrrr}3 & 1 & 6 & 42 & 62 & -17 \\ 4 & 1 & 6 & 128 & -26 & -8\end{array}$

$\begin{array}{llllll}5 & 1 & 6 & 199 & -186 & 6\end{array}$

$\begin{array}{rrrrrr}6 & 1 & 6 & 117 & 77 & 9 \\ 7 & 1 & 6 & 149 & -64 & -8\end{array}$

$\begin{array}{rrrrrr}8 & 1 & 6 & 36 & 29 & -31 \\ 9 & 1 & 6 & 48 & -23 & -23\end{array}$

$\begin{array}{rrrrrr}9 & 1 & 6 & 48 & -23 & -23 \\ 10 & 8 & -17\end{array}$

$1116 \quad 37 \quad 3-39$

$\begin{array}{rrrrrr}13 & 1 & 6 & 38 & 29 & -40 \\ -10 & 2 & 6 & 44 & 40 & -39\end{array}$

$\begin{array}{rrrrrr}-10 & 2 & 6 & 44 & 40 & -39 \\ -9 & 2 & 6 & 110 & -87 & 24\end{array}$

$\begin{array}{llllll}-8 & 2 & 6 & 72 & 84 & -20\end{array}$

$\begin{array}{rrrrrr}-7 & 2 & 5 & 153 & -150 & 11 \\ -6 & 2 & 6 & 211 & 195 & 7\end{array}$ h K I 1OFO 1OFC IOS

$\begin{array}{rrrrrr}-5 & 2 & 6 & 164 & -152 & 7 \\ -4 & 2 & 6 & 424 & 443 & 6 \\ -3 & 2 & 6 & 589 & -571 & 6 \\ -2 & 2 & 6 & 409 & 415 & 6 \\ -1 & 2 & 6 & 781 & -820 & 5 \\ 0 & 2 & 5 & 947 & 1012 & 4 \\ 1 & 2 & 6 & 526 & -558 & 4 \\ 2 & 2 & 6 & 281 & 300 & 4 \\ 3 & 2 & 6 & 500 & -529 & 5 \\ 4 & 2 & 6 & 198 & 153 & 5 \\ 5 & 2 & 6 & 361 & -354 & 6 \\ 6 & 2 & 6 & 473 & 471 & 6 \\ 7 & 2 & 6 & 265 & -284 & 6 \\ 8 & 2 & 6 & 70 & 38 & -15 \\ 9 & 2 & 6 & 95 & -60 & 13\end{array}$

$\begin{array}{rrrrrr}9 & 2 & 6 & 95 & -60 & 13 \\ 10 & 2 & 6 & 118 & 83 & 12\end{array}$

$\begin{array}{rrrrrr}11 & 2 & 6 & 47 & -12 & -31 \\ 12 & 2 & 6 & 38 & 21 & -31\end{array}$

$\begin{array}{llllll}13 & 2 & 6 & 80 & -6 & -20\end{array}$

$\begin{array}{rrrrrr}-10 & 3 & 6 & 26 & 7 & -46 \\ -9 & 3 & 6 & 42 & -29 & -36\end{array}$

$\begin{array}{rrrrrr}-8 & 3 & 5 & 63 & -13 & -26 \\ -7 & 3 & 6 & 59 & 14 & -27\end{array}$

$\begin{array}{llllll}-6 & 3 & 6 & 66 & 60 & -18\end{array}$

$\begin{array}{rrrrrr}-5 & 3 & 6 & 79 & -67 & 14 \\ -4 & 3 & 6 & 201 & -210 & 6\end{array}$

$\begin{array}{rrrrr}-3 & 3 & 6 & 202 & 197 \\ -2 & 3 & 6 & 94 & -102\end{array}$

$\begin{array}{llllll}-1 & 3 & 6 & 477 & -434 & 5\end{array}$

$\begin{array}{llllll}0 & 3 & 6 & 41 & 58 & -22\end{array}$

$\begin{array}{rrrrrr}1 & 3 & 6 & 128 & -90 & 6 \\ 2 & 3 & 6 & 80 & -81 & 9\end{array}$

$\begin{array}{rrrrrr}4 & 3 & 6 & 195 & 157 & 5 \\ 5 & 3 & 6 & 165 & -167 & 6\end{array}$

$\begin{array}{llllll}6 & 3 & 6 & 161 & 161 & 7\end{array}$

$\begin{array}{rrrrrr}7 & 3 & 6 & 185 & 158 & 7 \\ 8 & 3 & 6 & 73 & -62 & -17\end{array}$

$\begin{array}{rrrrrr}9 & 3 & 6 & 117 & -100 & 12 \\ 10 & 3 & 6 & 46 & 11 & -35\end{array}$

$1136 \quad 61 \quad 50-29$

$\begin{array}{llllll}12 & 3 & 6 & 60 & -22 & -27\end{array}$

$\begin{array}{rrrrrr}13 & 3 & 6 & 26 & 26 & -48 \\ -10 & 4 & 6 & 53 & 58 & -30\end{array}$

$\begin{array}{llllll}-9 & 4 & 6 & 25 & -72 & -46\end{array}$

$\begin{array}{rrrrrr}-8 & 4 & 6 & 24 & 45 & -44 \\ -7 & 4 & 6 & 128 & -117 & 12\end{array}$

$\begin{array}{rrrrrr}-6 & 4 & 6 & 205 & 246 & 9 \\ -5 & 4 & 6 & 199 & -201 & 7\end{array}$

$\begin{array}{lllll}-5 & 4 & 6 & 199 & -201 \\ -4 & 4 & 6 & 217 & 223\end{array}$

$\begin{array}{lllll}-3 & 4 & 6 & 468 & -471\end{array}$

$\begin{array}{lllll}-2 & 4 & 6 & 466 & 475\end{array}$

$\begin{array}{lllll}-1 & 4 & 6 & 390 & -391\end{array}$

$\begin{array}{rrrrr}1 & 4 & 6 & 570 & 552 \\ 1 & 4 & 6 & 500 & -823\end{array}$

$246500 \quad 475$

$\begin{array}{lllll}3 & 4 & 6 & 582 & -584\end{array}$

$\begin{array}{rrrrr}4 & 4 & 5 & 340 & 350 \\ 5 & 4 & 6 & 203 & -190\end{array}$

$\begin{array}{rrrrr}6 & 4 & 6 & 279 & 273 \\ 7 & 4 & 6 & 177 & -187\end{array}$

$\begin{array}{rrrrrr}8 & 4 & 6 & 177 & -187 & 7 \\ 9 & 141 & 145 & 9\end{array}$

$946 \quad 59 \quad-28-25$

$\begin{array}{rrrrrr}10 & 4 & 6 & 115 & 127 & 14 \\ 11 & 4 & 6 & 33 & -71 & -40\end{array}$

$\begin{array}{llllll}12 & 4 & 6 & 42 & 53 & -40 \\ 13 & 4 & 6 & 32 & 22 & -46\end{array}$

$\begin{array}{llllll}-9 & 5 & 6 & 49 & 12 & -37\end{array}$

$\begin{array}{llllll}-8 & 5 & 6 & 76 & -46 & -20\end{array}$

$-756 \quad 85 \quad 95-28$

$\begin{array}{rrrrrr}-6 & 5 & 6 & 62 & -16 & -27 \\ -5 & 5 & 6 & 92 & -54 & 24\end{array}$

$\begin{array}{llllll}-4 & 5 & 6 & 43 & 53 & -29\end{array}$

$\begin{array}{llllll}-3 & 5 & 6 & 58 & 64 & -16\end{array}$

$\begin{array}{rrrrrr}-2 & 5 & 6 & 223 & -199 & 6 \\ -1 & 5 & 6 & 10 & 13 & -40\end{array}$

$\begin{array}{rrrrrr}-1 & 5 & 6 & 10 & 13 & -40 \\ 0 & 5 & 6 & 84 & 72 & 9\end{array}$

$\begin{array}{llllll}1 & 5 & 6 & 64 & -15 & 11\end{array}$

$\begin{array}{llllll}2 & 5 & 6 & 380 & -382 & 5\end{array}$

$\begin{array}{rrrrrr}3 & 5 & 6 & 84 & -58 & 10 \\ 4 & 5 & 6 & 156 & -58 & -7\end{array}$

$\begin{array}{lllll}4 & 5 & 6 & 156 & -68 \\ 5 & 5 & 6 & 270 & -271\end{array}$

$\begin{array}{llllr}6 & 5 & 6 & 237 & 216 \\ 7 & 5 & 6 & 123 & -77\end{array}$

$\begin{array}{llllll}7 & 5 & 6 & 123 & -77 & 1 \\ 8 & 5 & 6 & 264 & -280 & \end{array}$

$\begin{array}{llllll}9 & 5 & 6 & 74 & -27 & -16\end{array}$

$\begin{array}{rrrrrr}10 & 5 & 6 & 83 & 4 & -18 \\ 11 & 5 & 6 & 37 & 21 & -38\end{array}$

$\begin{array}{llllll}12 & 5 & 6 & 71 & 7 & -22\end{array}$

$\begin{array}{rrrrrr}13 & 5 & 6 & 75 & -2 & -22 \\ -9 & 6 & 6 & 27 & -69 & -51\end{array}$

h $\mathrm{k}$ I IOFO 1OFC $10 \mathrm{~s}$

$\begin{array}{llllll}-8 & 6 & 6 & 90 & 88 & -19\end{array}$

$\begin{array}{rrrrrr}-7 & 6 & 6 & 91 & -79 & 18 \\ -6 & 6 & 6 & 167 & 165 & 10\end{array}$

$\begin{array}{llllll}-5 & 6 & 6 & 53 & -50 & -28\end{array}$

$\begin{array}{rrrrrr}-4 & 6 & 6 & 62 & 44 & -17 \\ -3 & 6 & 6 & 254 & -239 & 6\end{array}$

$\begin{array}{rrrrr}-3 & 6 & 6 & 254 & -239 \\ -2 & 6 & 6 & 658 & 651\end{array}$

$\begin{array}{lllll}-1 & 6 & 6 & 616 & -552\end{array}$

- $6 \quad 6 \quad 632 \quad 592$

$\begin{array}{lllll}1 & 6 & 6 & 399 & -391 \\ 2 & 6 & 6 & 459 & 443\end{array}$

$\begin{array}{rrrrr}2 & 6 & 6 & 459 & 441 \\ 3 & 6 & 6 & 442 & -445\end{array}$

$46 \quad 6 \quad 440 \quad 481$

666314301

$76 \quad 6 \quad 225-212 \quad 7$

$\begin{array}{rrrrrr}8 & 6 & 6 & 258 & 261 & 7 \\ 9 & 6 & 6 & 167 & -179 & 11\end{array}$

$10 \quad 6 \quad 6 \quad 82 \quad 2-17$

$\begin{array}{rrrrrr}11 & 6 & 6 & 64 & 0 & -29 \\ 12 & 6 & 6 & 95 & 47 & 17\end{array}$

$\begin{array}{rrrrrr}12 & 6 & 6 & 95 & 47 & 17 \\ 13 & 6 & 6 & 79 & -52 & -22 \\ -8 & 7 & 6 & 46 & -48 & -39\end{array}$

$\begin{array}{llllll}-7 & 7 & 6 & 77 & 66 & -20\end{array}$

$\begin{array}{rrrrrr}-5 & 7 & 6 & 51 & 52 & -30 \\ -4 & 7 & 6 & 102 & -72 & 13\end{array}$

$\begin{array}{rrrrrr}-3 & 7 & 6 & 70 & 93 & -17 \\ -2 & 7 & 6 & 311 & -311 & 6\end{array}$

$\begin{array}{llllll}-1 & 7 & 6 & 145 & 207 & 8\end{array}$

$\begin{array}{rrrrrr}0 & 7 & 6 & 45 & 3 & -25 \\ 1 & 7 & 6 & 287 & -186 & 6\end{array}$

276 118 -1008

$\begin{array}{llllll}3 & 7 & 6 & 75 & 49 & 12 \\ 4 & 7 & 6 & 94 & 42 & 10\end{array}$

$\begin{array}{llllll}5 & 7 & 6 & 44 & -29 & -28\end{array}$

$\begin{array}{llllll}6 & 7 & 6 & 114 & 64 & 11\end{array}$

$\begin{array}{rrrrrr}7 & 7 & 6 & 132 & -114 & 10 \\ 8 & 7 & 6 & 62 & -29 & -20\end{array}$

$97 \quad 6 \quad 93 \quad-49 \quad 15$

$1076 \quad 84 \quad 210-18$

$\begin{array}{llllll}11 & 7 & 6 & 94 & -30 & 15\end{array}$

$\begin{array}{rrrrrr}12 & 7 & 6 & 40 & -41 & -41 \\ 33 & 7 & 6 & 56 & 25 & -36\end{array}$

$\begin{array}{rrrrrr}-7 & 8 & 6 & 74 & -73 & -30\end{array}$

$\begin{array}{llllll}-5 & 8 & 6 & 177 & -170 & 11\end{array}$

$\begin{array}{rrrrrr}-4 & 8 & 6 & 194 & 184 & 9 \\ -3 & 8 & 6 & 130 & -113 & 11\end{array}$

$\begin{array}{rrrrr}-3 & 8 & 6 & 130 & -113 \\ -2 & 8 & 6 & 246 & 250\end{array}$

$\begin{array}{lllll}-1 & 8 & 6 & 372 & -360\end{array}$

$\begin{array}{llllll}0 & 8 & 6 & 201 & 156\end{array}$

$1865434-440$ 
h $K 1$ IOFO LOFC lOS

$\begin{array}{llllll}1 & 10 & 6 & 409 & -399 & 7\end{array}$ $210 \quad 6 \quad 244 \quad 232$ $\begin{array}{llllll}3 & 10 & 6 & 251 & -259 & 7\end{array}$ $\begin{array}{lllll}40 & 6 & 23 & 52 & -33\end{array}$ $510629 \quad 2-29$ $6106 \quad 66 \quad 67-19$

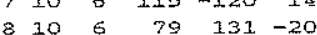
$9 \begin{array}{lllll}90 & 6 & 99 & -123 & 17\end{array}$ $1010 \quad 6 \quad 54 \quad 61-33$

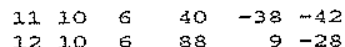
$\begin{array}{lllll}11 & 5 & 62 & -39 & -32\end{array}$ $\begin{array}{llllll}-3 & 11 & 6 & 81 & 33 & -19\end{array}$ $\begin{array}{llllll}-2 & 11 & 6 & 38 & -13 & -37 \\ -1 & 11 & 6 & 47 & -39 & -34\end{array}$ - $11650 \quad 6-30$ $\begin{array}{llllll}1 & 1 . & 5 & 89 & -86 & 15\end{array}$ $\begin{array}{llllll}211 & 5 & 166 & 171 & 10\end{array}$ $\begin{array}{llllll}311 & 6 & 145 & -138 & 11\end{array}$ $\begin{array}{rrrrrr}4 & 11 & 6 & 154 & 162 & 9 \\ 5 & 11 & 6 & 110 & -87 & 12\end{array}$ $6116 \quad 130 \quad 106 \quad 11$ $\begin{array}{llllll}711 & 5 & 45 & -33 & -34\end{array}$ $811655 \quad 6-30$ $\begin{array}{llllll}9 & 11 & 6 & 71 & -38 & -21\end{array}$ $\begin{array}{llllll}11 & 11 & 6 & 37 & 17 & -37 \\ 17 & -44\end{array}$ $12116 \quad 11-44-46$ $\begin{array}{llllll}-3 & 12 & 6 & 152 & -163 & 13\end{array}$ $\begin{array}{rrrrrr}-2 & 12 & 6 & 154 & 146 & 13 \\ -1 & 12 & 6 & 119 & -118 & 14\end{array}$ $\begin{array}{llllll}0 & 12 & 6 & 123 & 134 & 14\end{array}$ $\begin{array}{llllll}1 & 12 & 6 & 202 & -198 & 10\end{array}$ $212 \quad 6 \quad 94 \quad 117 \quad 16$ $\begin{array}{rrrrrr}3 & 12 & 6 & 220 & -213 & 8 \\ 4 & 12 & 6 & 192 & 209 & 9\end{array}$ $\begin{array}{llllll}5 & 12 & 6 & 151 & -171 & 12\end{array}$ $\begin{array}{rrrrrr}6 & 12 & 6 & 124 & 127 & 14 \\ 7 & 12 & 6 & 64 & -7 & -29\end{array}$

$\begin{array}{rrrrrr}7 & 12 & 6 & 64 & -7 & -29 \\ \mathrm{~s} & 12 & 6 & 103 & 62 & 15\end{array}$ $\begin{array}{llllll}912 & 6 & 31 & -55 & -43 \\ 1 & 12 & 6 & 26 & 71 & -51\end{array}$ $\begin{array}{llllll}11 & 12 & 6 & 50 & -49 & -38\end{array}$ $\begin{array}{llllll}-1 & 13 & 6 & 89 & -65 & -19\end{array}$

- $136 \quad 15 \quad 102-59$

$\begin{array}{rrrrrr}1 & 13 & 6 & 49 & -57 & -29 \\ 2 & 13 & 6 & 58 & 30 & -32\end{array}$

$\begin{array}{llllll}3 & 13 & 6 & 100 & -86 & 15\end{array}$

$\begin{array}{llllll}4 & 13 & 6 & 63 & 59 & -28\end{array}$

$\begin{array}{llllll}5 & 13 & 6 & 99 & -77 & 14\end{array}$

$\begin{array}{rrrrrr}6 & 13 & 6 & 70 & 57 & -21 \\ 7 & 13 & 6 & 108 & -112 & 17\end{array}$

$8136 \quad 204 \quad 50 \quad 16$

$\begin{array}{llllll}9 & 13 & 6 & 76 & -11 & -32\end{array}$

$\begin{array}{llllll}10 & 13 & 6 & 54 & 17 & -37\end{array}$

$\begin{array}{rrrrrr}1 & 14 & 6 & 103 & -117 & 18 \\ 2 & 14 & 6 & 48 & 141 & -41\end{array}$

$\begin{array}{llllll}3 & 14 & 6 & 121 & -131 & 15\end{array}$

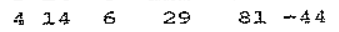

$\begin{array}{llllll}5 & 3.4 & 6 & 24 & -63 & -46\end{array}$

$\begin{array}{rrrrrr}6 & 14 & 6 & 102 & 114 & 18 \\ 7 & 14 & 6 & 68 & -100 & -32\end{array}$

$\begin{array}{llllrr}7 & 14 & 6 & 68 & -100 & -32 \\ 8 & 14 & 6 & 74 & 39 & -22\end{array}$

$\begin{array}{llllll}914 & 6 & 33 & -57 & -46\end{array}$

$\begin{array}{lllll}-6-12 & 7 & 72 & 38 & -20\end{array}$

$\begin{array}{lllll}-5-32 & 7 & 54 & -64 & -33\end{array}$

$\begin{array}{rrrrr}-4-12 & 7 & 34 & 43 & -41 \\ -3-12 & 7 & 51 & -32 & -32\end{array}$

$\begin{array}{lllll}-2-12 & 7 & 63 & 47 & -30\end{array}$

$\begin{array}{rrrrr}-1-12 & 7 & 47 & -113 & -40 \\ 0-12 & 7 & 90 & 98 & -18\end{array}$

$\begin{array}{lllll}1-12 & 7 & 79 & -37 & -20\end{array}$

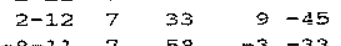

$\begin{array}{lllll}-8-11 & 7 & 58 & -3 & -33 \\ -7-11 & 7 & 87 & -22 & -18\end{array}$

$\begin{array}{lllll}-6-11 & 7 & 91 & 92 & -19\end{array}$

$\begin{array}{lllll}-5-11 & 7 & 14 & -97 & -54\end{array}$

$\begin{array}{lllll}-4-11 & 7 & 238 & 111 & 11 \\ -3-11 & 7 & 130 & -141 & 12\end{array}$

$\begin{array}{lllll}-2-11 & 7 & 123 & 128 & 12\end{array}$

$-3-117100-115 \quad 16$

$\begin{array}{lllll}0-13 & 7 & 97 & 127 & 16\end{array}$

$\begin{array}{lllll}1-11 & 7 & 168 & -168 & 12\end{array}$

$\begin{array}{lllll}2-11 & 7 & 167 & 173 & 13\end{array}$

$\begin{array}{rrrrr}3-11 & 7 & 144 & -150 & 14 \\ 4-11 & 7 & 120 & 110 & 17\end{array}$

$\begin{array}{rrrrr}4-11 & 7 & 120 & 110 & 17 \\ -9-10 & 7 & 14 & -34 & -55\end{array}$

$\begin{array}{rrrrr}-8-10 & 7 & 25 & 18 & -46 \\ -7-10 & 7 & 78 & -34 & -19\end{array}$ h $k$ I IOFO IOFC 105

$\begin{array}{rrrrr}-6-10 & 7 & 54 & 19 & -23 \\ -5-10 & 7 & 58 & -45 & -27\end{array}$

$\begin{array}{lllll}-4-10 & 7 & 117 & 110 & 12\end{array}$

$\begin{array}{lllll}-3-10 & 7 & 132 & -138 & 11\end{array}$

$\begin{array}{rrrrr}-2-10 & 7 & 137 & 123 & 11 \\ -1-10 & 7 & 97 & -63 & 15\end{array}$

$\begin{array}{rrrrr}0-10 & 7 & 39 & 50 & -35\end{array}$

$1-10 \quad 7 \quad 40 \quad-31-34$

$\begin{array}{lllll}2-10 & 7 & 12 & -11 & -48\end{array}$

$\begin{array}{rrrrr}3-10 & 7 & 13 & -30 & -54 \\ 4-10 & 7 & 113 & 46 & -24\end{array}$

$\begin{array}{rrrrr}5-10 & 7 & 115 & -43 & 16\end{array}$

$\begin{array}{llllll}-10 & -9 & 7 & 70 & 36 & -31\end{array}$

$\begin{array}{llllll}-9 & -9 & 7 & 73 & -46 & -22\end{array}$

$\begin{array}{llllll}-8 & -9 & 7 & 54 & 59 & -31\end{array}$

$\begin{array}{rrrrrr}-7 & -9 & 7 & 22 & -31 & -39 \\ -5 & -9 & 7 & 83 & 22 & 15\end{array}$

$\begin{array}{llllll}-4 & -9 & 7 & 76 & 20 & -21\end{array}$

$\begin{array}{llllll}-3 & -9 & 7 & 150 & -147 & 10\end{array}$

$\begin{array}{rrrrrr}-2 & -9 & 7 & 205 & 232 & 8\end{array}$

$\begin{array}{lllll}-1 & -9 & 7 & 320 & -315 \\ 0 & -9 & 7 & 318 & 322\end{array}$

$\begin{array}{rrrrrr}0 & -9 & 7 & 318 & 322 & 7 \\ 1 & -9 & 7 & 198 & -199 & 9\end{array}$

$\begin{array}{llllll}2 & -9 & 7 & 174 & 191 & 11\end{array}$

$\begin{array}{llllll}3 & -9 & 7 & 165 & -177 & 11\end{array}$

$\begin{array}{rrrrrr}4 & -9 & 7 & 142 & 138 & 13 \\ 5 & -9 & 7 & 125 & -139 & 16\end{array}$

$\begin{array}{llllll}6 & -9 & 7 & 152 & 167 & 14\end{array}$

$\begin{array}{llllll}-10 & -8 & 7 & 61 & 24 & -29\end{array}$

$\begin{array}{llllll}-9 & -8 & 7 & 43 & -34 & -36\end{array}$

$\begin{array}{rrrrrr}-8 & -8 & 7 & 36 & 45 & -31 \\ -7 & -8 & 7 & 89 & -51 & 15\end{array}$

$\begin{array}{rrrrrr}-7 & -8 & 7 & 89 & -51 & 15 \\ -6 & -8 & 7 & 80 & 76 & -16\end{array}$

$\begin{array}{llllll}-5 & -8 & 7 & 85 & -94 & 16\end{array}$

$\begin{array}{rrrrrr}-4 & -8 & 7 & 76 & 17 & -15 \\ -3 & -8 & 7 & 136 & 86 & 10\end{array}$

$\begin{array}{llllll}-2 & -8 & 7 & 27 & -71 & -37\end{array}$

$\begin{array}{lllllll}-1 & -8 & 7 & 45 & -48 & -25\end{array}$

$\begin{array}{llllll}0 & -8 & 7 & 34 & 3.2 & -36\end{array}$

$\begin{array}{llllll}1 & -8 & 7 & 175 & -186 & 10\end{array}$

$\begin{array}{rrrrrr}2 & -8 & 7 & 128 & 99 & 12 \\ 3 & -8 & 7 & 31 & 11 & -37\end{array}$

$\begin{array}{llllll}4 & -8 & 7 & 53 & -1 & -30\end{array}$

$\begin{array}{llllll}6 & -8 & 7 & 43 & -18 & -37\end{array}$

$\begin{array}{llllll}7 & -8 & 7 & 25 & 32 & -49\end{array}$

$\begin{array}{rrrrrr}8 & -8 & 7 & 64 & 9 & -35 \\ -10 & -7 & 7 & 36 & 30 & -40\end{array}$

$\begin{array}{llllll}-9 & -7 & 7 & 100 & -75 & 14\end{array}$

$\begin{array}{llllll}-8 & -7 & 7 & 84 & 78 & -18\end{array}$

$\begin{array}{llllll}-7 & -7 & 7 & 84 & -99 & 16\end{array}$

$\begin{array}{llllll}-6 & -7 & 7 & 132 & 1.44 & 12\end{array}$

$\begin{array}{llllll}-5 & -7 & 7 & 153 & -165 & 10\end{array}$

$\begin{array}{rrrrrr}-4 & -7 & 7 & 158 & 159 & 8 \\ -3 & -7 & 7 & 288 & -296 & 6\end{array}$

$\begin{array}{llllll}-3 & -7 & 7 & 288 & -296 & 6 \\ -2 & -7 & 7 & 205 & 198 & 7\end{array}$

$\begin{array}{rrrrrr}-1 & -7 & 7 & 98 & -66 & 11 \\ 0 & -7 & 7 & 166 & 150 & 7\end{array}$

$\begin{array}{lllll}0 & -7 & 7 & 166 & 150\end{array}$

$\begin{array}{rrrrr}1 & -7 & 7 & 245 & -248 \\ 2 & -7 & 7 & 263 & 267\end{array}$

$\begin{array}{llllll}3 & -7 & 7 & 361 & -368 & 7\end{array}$

$\begin{array}{llllll}4 & -7 & 7 & 262 & 256\end{array}$

$\begin{array}{llllll}5 & -7 & 7 & 148 & -159 & 12\end{array}$

$\begin{array}{llllll}6 & -7 & 7 & 170 & 204 & 11\end{array}$

$\begin{array}{llllll}7 & -7 & 7 & 171 & -162 & 10\end{array}$

$\begin{array}{rrrrrr}-11 & -6 & 7 & 32 & -3 & -42 \\ -10 & -6 & 7 & 31 & 22 & -45\end{array}$

$\begin{array}{rrrrrr}-10 & -6 & 7 & 31 & 22 & -45 \\ -9 & -6 & 7 & 103 & -36 & 14\end{array}$

$\begin{array}{rrrrrr}-8 & -6 & 7 & 87 & 52 & 17\end{array}$

$\begin{array}{llllll}-6 & -6 & 7 & 28 & 29 & -38\end{array}$

$\begin{array}{llllll}-5 & -6 & 7 & 53 & -41 & -25\end{array}$

$\begin{array}{rrrrrr}-4 & -6 & 7 & 77 & 78 & 13\end{array}$

$\begin{array}{rrrrrr}-3 & -6 & 7 & 177 & -198 & 7 \\ -2 & -6 & 7 & 138 & 129 & 8\end{array}$

$\begin{array}{llllll}-1 & -6 & 7 & 128 & -158 & 9\end{array}$

$\begin{array}{llllll}0 & -6 & 7 & 332 & 141 & 8\end{array}$

$\begin{array}{rrrrrr}1 & -6 & 7 & 326 & 128 & 9 \\ 2 & -6 & 7 & 261 & -142 & 8\end{array}$

$\begin{array}{llllll}3 & -6 & 7 & 44 & 22 & -19\end{array}$

$\begin{array}{llllll}4 & -6 & 7 & 50 & -27 & -28 \\ 5 & -6 & 7 & 56 & -65 & -28\end{array}$

$\begin{array}{rrrrrr}5 & -6 & 7 & 56 & -65 & -28 \\ 6 & -6 & 7 & 32 & 6 & -40\end{array}$

$7-67 \quad 86 \quad 102-18$

$\begin{array}{llllll}8 & -6 & 7 & 55 & -23 & -32 \\ 9 & -6 & 7 & 42 & -26 & -39\end{array}$

$\begin{array}{llllll}-11 & -5 & 7 & 41 & -22 & -35\end{array}$

$\begin{array}{rrrrrr}-10 & -5 & 7 & 88 & 35 & -23 \\ -9 & -5 & 7 & 66 & -77 & -20\end{array}$

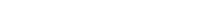

$\begin{array}{rrrrrr}-8 & -5 & 7 & 98 & 80 & 14 \\ -7 & -5 & 7 & 107 & -43 & 11 \\ -6 & -5 & 7 & 94 & 104 & 14 \\ -5 & -5 & 7 & 163 & -151 & 8 \\ -4 & -5 & 7 & 109 & 106 & 10 \\ -3 & -5 & 7 & 240 & -257 & 6 \\ -2 & -5 & 7 & 184 & 170 & 6 \\ -1 & -5 & 7 & 419 & -433 & 6 \\ 0 & -5 & 7 & 604 & 597 & 6 \\ 1 & -5 & 7 & 466 & -432 & 6 \\ 2 & -5 & 7 & 327 & 321 & 5 \\ 3 & -5 & 7 & 195 & -205 & 7 \\ 4 & -5 & 7 & 118 & 93 & 10 \\ 5 & -5 & 7 & 177 & -170 & 8 \\ 6 & -5 & 7 & 203 & 170 & 8 \\ 7 & -5 & 7 & 158 & -164 & 11 \\ 8 & -5 & 7 & 123 & 99 & 11 \\ 9 & -5 & 7 & 73 & -39 & -19\end{array}$

$\begin{array}{rrrrrr}9 & -5 & 7 & 73 & -39 & -19 \\ 10 & -5 & 7 & 59 & 0 & -32\end{array}$

$\begin{array}{rrrrrr}-21 & -4 & 7 & 41 & -13 & -42 \\ -10 & -4 & 7 & 62 & 26 & -29\end{array}$

$\begin{array}{rrrrrr}-10 & -4 & 7 & 62 & 26 & -29 \\ -9 & -4 & 7 & 74 & -56 & -18\end{array}$

$\begin{array}{rrrrrr}-8 & -4 & 7 & 55 & 25 & -28\end{array}$

$\begin{array}{rrrrrr}-7 & -4 & 7 & 97 & -116 & 13 \\ -6 & -4 & 7 & 186 & 180 & 7\end{array}$

$\begin{array}{rrrrrr}-6 & -4 & 7 & 186 & 180 & 7 \\ -5 & -4 & 7 & 79 & -87 & 14\end{array}$

$\begin{array}{rrrrrr}-4 & -4 & 7 & 66 & 91 & -15 \\ -3 & -4 & 7 & 99 & 42 & 9\end{array}$

$\begin{array}{llllll}-2 & -4 & 7 & 170 & -3.73 \quad 6\end{array}$

$\begin{array}{lllllll}-1 & -4 & 7 & 142 & 151 & 7\end{array}$

$\begin{array}{rrrrrr}0 & -4 & 7 & 28 & 18 & -26 \\ 1 & -4 & 7 & 61 & 37 & -13\end{array}$

$\begin{array}{rrrrrr}2 & -4 & 7 & 1.29 & 112 & 8\end{array}$

$\begin{array}{llllll}4 & -4 & 7 & 72 & -13 & 14\end{array}$

$\begin{array}{rrrrrr}4 & -4 & 7 & 72 & -13 & 14 \\ 5 & -4 & 7 & 71 & 18 & -14\end{array}$

$\begin{array}{llllll}6 & -4 & 7 & 38 & 40 & -32\end{array}$

$\begin{array}{rrrrrr}7 & -4 & 7 & 88 & 21 & 15 \\ 8 & -4 & 7 & 60 & -81 & -28\end{array}$

$\begin{array}{llllll}9 & -4 & 7 & 45 & 48 & -33\end{array}$

$\begin{array}{llllll}10 & -4 & 7 & 79 & -14 & -19\end{array}$

$\begin{array}{rrrrrr}11 & -4 & 7 & 104 & 36 & -23 \\ -11 & -3 & 7 & 99 & -71 & 16\end{array}$

$\begin{array}{rrrrrr}-11 & -3 & 7 & 99 & -71 & 16 \\ -10 & -3 & 7 & 64 & 62 & -29\end{array}$

$\begin{array}{llllll}-9 & -3 & 7 & 106 & -105 & 34\end{array}$

$\begin{array}{rrrrrr}-8 & -3 & 7 & 84 & 76 & 17 \\ -7 & -3 & 7 & 86 & -108 & 15\end{array}$

$\begin{array}{rrrrrr}-6 & -3 & 7 & 96 & 69 & 12\end{array}$

$\begin{array}{rrrrr}-5 & -3 & 7 & 201 & -222 \\ -4 & -3 & 7 & 304 & 320\end{array}$

$\begin{array}{lllll}-3 & -3 & 7 & 497 & -515\end{array}$

$\begin{array}{lllll}-2 & -3 & 7 & 480 & 492\end{array}$

$\begin{array}{lllll}-1 & -3 & 7 & 448 & -442\end{array}$

\begin{tabular}{rrrrr}
0 & -3 & 7 & 471 & 502 \\
\hline & -3 & 7 & 443 & -455
\end{tabular}

$\begin{array}{lllll}2 & -3 & 7 & 259 & 261\end{array}$

$\begin{array}{lllll}3 & -3 & 7 & 334 & -337\end{array}$

$\begin{array}{rrrrr}4 & -3 & 7 & 290 & 285 \\ 5 & -3 & 7 & 125 & -107\end{array}$

$\begin{array}{rrrrrr}5 & -3 & 7 & 125 & -107 & 9 \\ 6 & -3 & 7 & 192 & 172 & 8\end{array}$

$\begin{array}{llllll}7 & -3 & 7 & 137 & -94 & 11\end{array}$

$\begin{array}{llllll}8 & -3 & 7 & 89 & 98 & 16\end{array}$

$\begin{array}{llllll}9 & -3 & 7 & 100 & -140 & 15\end{array}$

$\begin{array}{rrrrrr}10 & -3 & 7 & 84 & 33 & 15\end{array}$

$\begin{array}{rrrrrr}12 & -3 & 7 & 2.17 & 9 & 19 \\ -11 & -2 & 7 & 61 & 4 & -32\end{array}$

$\begin{array}{llllll}-10 & -2 & 7 & 70 & -14 & -22\end{array}$

$\begin{array}{rrrrrr}-9 & -2 & 7 & 58 & -35 & -28 \\ -8 & -2 & 7 & 63 & 15 & -19\end{array}$

$\begin{array}{rrrrrr}-7 & -2 & 7 & 60 & 51 & -25 \\ -6 & -2 & 7 & 74 & -29 & 14\end{array}$

$\begin{array}{llllll}-5 & -2 & 7 & 27 & -28 & -33\end{array}$

$\begin{array}{rrrrrr}-5 & -2 & 7 & 51 & 22 & -15\end{array}$

$\begin{array}{rrrrrr}-3 & -2 & 7 & 87 & -105 & 10 \\ -2 & -2 & 7 & 34 & -27 & -23\end{array}$

$\begin{array}{rrrrrr}-2 & -2 & 7 & 34 & -27 & -23 \\ -1 & -2 & 7 & 81 & -14 & 13\end{array}$

$\begin{array}{lllllll}0 & -2 & 7 & 422 & 434 & 5\end{array}$

$\begin{array}{llllll}1 & -2 & 7 & 223 & -202 & 5\end{array}$

$\begin{array}{rrrrrr}2 & -2 & 7 & 28 & -10 & -28 \\ 3 & -2 & 7 & 245 & -238 & 5\end{array}$

$\begin{array}{rrrrrr}4 & -2 & 7 & 81 & 7 & 11 \\ 5 & -2 & 7 & 147 & -107 & 7\end{array}$

$\begin{array}{llllll}6 & -2 & 7 & 177 & 153 & 7\end{array}$

$\begin{array}{rrrrrr}7 & -2 & 7 & 64 & -30 & -16 \\ 8 & -2 & 7 & 51 & 7 & -28\end{array}$

$\begin{array}{rrrrrr}9 & -2 & 7 & 69 & 32 & -19 \\ 10 & -2 & 7 & 66 & -9 & -21\end{array}$

$\begin{array}{rrrrrr}10 & -2 & 7 & 66 & -9 & -21 \\ 11 & -2 & 7 & 51 & 2 & -33\end{array}$

$\begin{array}{llllll}12 & -2 & 7 & 80 & 9 & -28\end{array}$ h $k 1$ IOFO IOFC $10 \mathrm{~s}$

$\begin{array}{llllll}-10 & -1 & 7 & 59 & 66 & -24\end{array}$

$\begin{array}{lllllll}-9 & -1 & 7 & 110 & -109 & 14\end{array}$

$\begin{array}{llllll}-8 & -1 & 7 & 115 & 120 & 13 \\ -7 & -1 & 7 & 118 & -95 & 11\end{array}$

$\begin{array}{lllll}-6 & -1 & 7 & 250 & 251\end{array}$

$\begin{array}{lllll}-5 & -1 & 7 & 155 & -160\end{array}$

$\begin{array}{rrrrr}-4 & -1 & 7 & 93 & 83 \\ -3 & -1 & 7 & 218 & -222\end{array}$

$\begin{array}{llllll}-2 & -1 & 7 & 32 & 92 & -19\end{array}$

$\begin{array}{llllll}-1 & -1 & 7 & 517 & -558\end{array}$

$\begin{array}{rrrrr}0 & -1 & 7 & 324 & 888 \\ 1 & -1 & 7 & 609 & -652\end{array}$

$\begin{array}{rrrrr}1 & -1 & 7 & 609 & -652 \\ 2 & -1 & 7 & 448 & 473\end{array}$

$\begin{array}{lllll}3 & -1 & 7 & 317 & -317\end{array}$

$\begin{array}{llll}-1 & 7 & 303 & 307\end{array}$

$\begin{array}{lllll}5 & -1 & 7 & 490 & -515\end{array}$

$\begin{array}{rrrrr}6 & -1 & 7 & 402 & 400 \\ 7 & -1 & 7 & 255 & -262\end{array}$

$\begin{array}{llllll}8 & -1 & 7 & 143 & 108 & 10\end{array}$

$\begin{array}{llllll}9 & -2 & 7 & 122 & -326 & 13\end{array}$

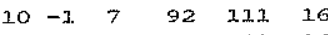

$\begin{array}{rrrrrr}11 & -1 & 7 & 62 & -29 & -26 \\ 12 & -1 & 7 & 11 & 57 & -43\end{array}$

$\begin{array}{llllll}-11 & 0 & 7 & 27 & 3 & -32\end{array}$

$\begin{array}{llllll}-10 & 0 & 7 & 64 & 2 & -19\end{array}$

$\begin{array}{rrrrrr}-9 & 0 & 7 & 94 & 27 & -22 \\ -8 & 0 & 7 & 92 & -31 & -23\end{array}$

$\begin{array}{rrrrrr}-8 & 0 & 7 & 92 & -31 & -23 \\ -7 & 0 & 7 & 85 & 9 & -18\end{array}$

$\begin{array}{rrrrrr}-6 & 0 & 7 & 68 & 18 & 11\end{array}$

$\begin{array}{rrrrrr}-5 & 0 & 7 & 108 & 114 & \\ -4 & 0 & 7 & 69 & 25 & -14\end{array}$

$\begin{array}{rrrrrr}-3 & 0 & 7 & 52 & 0 & -12 \\ -2 & 0 & 7 & 113 & -131 & 10\end{array}$

$\begin{array}{rrrrrr}-2 & 0 & 7 & 113 & -131 & 10 \\ -1 & 0 & 7 & 30 & 30 & -18\end{array}$

$\begin{array}{llllll}0 & 0 & 7 & 148 & -161 & 5 \\ 1 & 0 & 7 & 141 & -147 & 4\end{array}$

$\begin{array}{lllll}1 & 0 & 7 & 126 & 119\end{array}$

$\begin{array}{rrrrr}3 & 0 & 7 & 192 & -1.82 \\ 4 & 0 & 7 & 154 & 127\end{array}$

5007 121 81

$\begin{array}{rrrrrr}6 & 0 & 7 & 127 & 91 & 6 \\ 7 & 0 & 7 & 144 & -134 & 6\end{array}$

$\begin{array}{rrrrrr}7 & 0 & 7 & 144 & -134 & 6 \\ 8 & 0 & 7 & 88 & 132 & 10\end{array}$

$\begin{array}{rrrrrr}9 & 0 & 7 & 90 & -52 & 11\end{array}$

$\begin{array}{llllll}11 & 0 & 7 & 69 & 17 & -15\end{array}$

$\begin{array}{rlllrr}12 & 0 & 7 & 52 & 8 & -22 \\ 13 & 0 & 7 & 42 & -7 & -26\end{array}$

$\begin{array}{rrrrrr}-10 & 1 & 7 & 69 & 33 & -28\end{array}$

$\begin{array}{rrrrrr}-9 & 1 & 7 & 149 & -126 & 11 \\ -8 & 1 & 7 & 79 & 90 & -18\end{array}$

$\begin{array}{rrrrrr}-7 & 1 & 7 & 90 & -64 & 15 \\ -6 & 1 & 7 & 107 & 88 & 11\end{array}$

$\begin{array}{rrrrrr}-6 & 1 & 7 & 107 & 88 & 11 \\ -5 & 1 & 7 & 365 & -372 & 5\end{array}$

$\begin{array}{rrrrrr}-5 & 1 & 7 & 365 & -372 & 5 \\ -4 & 1 & 7 & 356 & 359 & 5\end{array}$

$\begin{array}{rrrrrr}-3 & 1 & 7 & 251 & -252 & 5 \\ -2 & 1 & 7 & 36 & 6 & -21\end{array}$

$\begin{array}{rrrrrr}-2 & 1 & 7 & 36 & 6 & -21 \\ -1 & 1 & 7 & 114 & -141 & 7\end{array}$

$\begin{array}{rrrlr}0 & 1 & 7 & 500 & 848 \\ 1 & 1 & 7 & 972-2040\end{array}$

$\begin{array}{lllll}2 & 7 & 673 & 715\end{array}$

$\begin{array}{llll}3 & 7 & 233 & -241\end{array}$

$\begin{array}{llll}1 & 7 & 181 & 179\end{array}$

$5 \quad 3 \quad 7 \quad 303-306$

$6 \quad 1 \quad 7 \quad 382 \quad 397$

\begin{tabular}{rrrr}
1 & 7 & 242 & -244 \\
\hline & 7 & 160 & 167
\end{tabular}

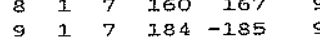

$10 \quad 1 \quad 7 \quad 158$ 171 11

$\begin{array}{rrrrrr}11 & 1 & 7 & 73 & -79 & -19 \\ 12 & 1 & 7 & 53 & 33 & -30\end{array}$

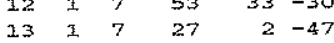

$\begin{array}{llllll}-10 & 2 & 7 & 32 & -25 & -44\end{array}$

$\begin{array}{llllll}-9 & 2 & 7 & 13 & -34 & -50 \\ -8 & 2 & 7 & 60 & -23 & -28\end{array}$

$\begin{array}{rrrrrr}-8 & 2 & 7 & 60 & -23 & -28 \\ -6 & 2 & 7 & 96 & 65 & 12\end{array}$

$\begin{array}{llllll}-5 & 2 & 7 & -45 & -15\end{array}$

$\begin{array}{rrrrrr}-4 & 2 & 7 & 161 & -161 & 7 \\ -3 & 2 & 7 & 96 & -99 & 10\end{array}$

$\begin{array}{rrrrrr}-3 & 2 & 7 & 96 & -99 & 20 \\ -2 & 2 & 7 & 42 & 25 & -22\end{array}$

$\begin{array}{rrrrrr}-1 & 2 & 7 & 42 & -50 & -16 \\ 0 & 2 & 7 & 273 & -253 & 4\end{array}$

$\begin{array}{rrrrrr}1 & 2 & 7 & 365 & 370 & 5 \\ 2 & 2 & 7 & 361 & -345 & 5\end{array}$

$\begin{array}{rrrrr}2 & 2 & 7 & 361 & -345 \\ 3 & 2 & 7 & 124 & 102\end{array}$

$\begin{array}{llllll}4 & 2 & 7 & 473 & 465 & 6 \\ 5 & 2 & 7 & 116 & 122 & 8\end{array}$

$\begin{array}{rrrrrr}6 & 2 & 7 & 74 & -9 & 13 \\ 7 & 2 & 7 & 134 & 79 & 8\end{array}$

$\begin{array}{lllrrr}8 & 2 & 7 & 50 & 59 & -26\end{array}$

h $\mathrm{k} 1$ 10FO 1OFC $10 \mathrm{~s}$

$\begin{array}{llllll}9 & 2 & 7 & 64 & -19\end{array}$

$\begin{array}{llllll}10 & 2 & 7 & 79 & 10 & -18 \\ 11 & 2 & 7 & 78 & 29 & -18 \\ 12 & 2 & 7 & 41 & 40 & -31\end{array}$

$\begin{array}{llllll}13 & 2 & 7 & 91 & -1 & -18\end{array}$

$\begin{array}{llllll}-10 & 3 & 7 & 42 & 45 & -38\end{array}$

$\begin{array}{rrrrrr}-9 & 3 & 7 & 91 & -81 & 17 \\ -7 & 3 & 7 & 143 & -129 & 10\end{array}$

$\begin{array}{llllll}-6 & 3 & 7 & 143 & 123 & 10\end{array}$

$\begin{array}{llllll}-5 & 3 & 7 & 256 & -259\end{array}$

$\begin{array}{lllll}-4 & 3 & 7 & 308 & 299\end{array}$

$\begin{array}{rrrrr}-3 & 3 & 7 & 428 & -442 \\ -2 & 3 & 7 & 426 & 435\end{array}$

$\begin{array}{lllll}-1 & 3 & 7 & 587 & -590\end{array}$

- 3710911090

$\begin{array}{rrrrr}1 & 3 & 7 & 999-1020 \\ 2 & 3 & 7 & 449 & 461\end{array}$

$\begin{array}{rrrrr}2 & 3 & 7 & 447 & 461 \\ 3 & 3 & 7 & 665 & -697\end{array}$

$\begin{array}{rrrrr}4 & 3 & 7 & 230 & 225 \\ 5 & 3 & 7 & 200 & -209\end{array}$

$\begin{array}{lllll}6 & 3 & 7 & 388 & 388\end{array}$

$\begin{array}{rrrrrr}7 & 3 & 7 & 229 & -226 & 6 \\ 8 & 3 & 7 & 52 & -15 & -23\end{array}$

\begin{tabular}{rrrrrr}
9 & 3 & 7 & 173 & -153 & 8 \\
\hline & 3 & 7 & 99 & 121 & 17
\end{tabular}

$\begin{array}{llllll}11 & 3 & 7 & 98 & -82 & 15\end{array}$

$\begin{array}{llllll}12 & 3 & 7 & 89 & 76 & 17\end{array}$

$\begin{array}{rrrrrr}13 & 3 & 7 & 32 & -7 & -45 \\ -9 & 4 & 7 & 40 & -28 & -37\end{array}$

$\begin{array}{llllll}-8 & 4 & 7 & 29 & -4 & -34 \\ -7 & 4 & 7 & 86 & 71 & 16\end{array}$

$\begin{array}{llllll}-6 & 4 & 7 & 42 & 33 & -31\end{array}$

$\begin{array}{rrrrrr}-5 & 4 & 7 & 86 & -105 & 14 \\ -4 & 4 & 7 & 83 & -73 & 14\end{array}$

$\begin{array}{rrrrrr}-3 & 4 & 7 & 251 & 234 & 6 \\ -2 & 4 & 7 & 238 & -252 & 6\end{array}$ 
h $k 1$ lOFo lOFC las

\begin{tabular}{|c|c|c|c|c|c|c|c|c|}
\hline 8 & 5 & 7 & 135 & -106 & 11 & 1 & 11 & 7 \\
\hline 9 & 6 & 7 & 59 & -27 & -22 & 2 & 11. & 7 \\
\hline 10 & 6 & 7 & 52 & 26 & -30 & 3 & 11 & 7 \\
\hline 11 & 6 & 7 & 47 & -10 & -32 & 4 & 12 & 7 \\
\hline 12 & 6 & 7 & 39 & 5 & -37 & 5 & 11 & 7 \\
\hline 13 & 6 & 7 & 49 & 5. & -35 & 6 & 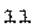 & 7 \\
\hline-8 & 7 & 7 & 50 & 44 & -34 & 7 & 11 & 7 \\
\hline-7 & 7 & 7 & 100 & -107 & 17 & 8 & 11 & 7 \\
\hline-6 & 7 & 7 & 133 & 159 & 14 & 9 & 11 & 7 \\
\hline-5 & 7 & 7 & 260 & -270 & 9 & 10 & 11 & 7 \\
\hline-4 & 7 & 7 & 59 & 87 & -27 & 11 & 11 & 7 \\
\hline-3 & 7 & 7 & 161 & -139 & 9 & 12 & 11 & 7 \\
\hline-2 & 7 & 7 & 340 & 330 & 6 & -3 & 12 & 7 \\
\hline-1 & 7 & 7 & 264 & -260 & 6 & -2 & 12 & 7 \\
\hline 0 & 7 & 7 & 588 & 540 & 7 & -1 & 12 & 7 \\
\hline 1 & 7 & 7 & 581 & -561 & 6 & 0 & 12 & 7 \\
\hline 2 & 7 & 7 & 339 & 314 & 5 & 1 & 12 & 7 \\
\hline 3 & 7 & 7 & 316 & -306 & 5 & 2 & 12 & 7 \\
\hline 4 & 7 & 7 & 236 & 255 & 6 & 3 & 12 & 7 \\
\hline 5 & 7 & 7 & 341 & -356 & 5 & 4 & 12 & 7 \\
\hline 6 & 7 & 7 & 366 & 377 & 6 & 5 & 12 & 7 \\
\hline 7 & 7 & 7 & 203 & -202 & 8 & 5 & 12 & 7 \\
\hline 8 & 7 & 7 & 173 & 198 & 9 & 7 & 1.2 & 7 \\
\hline 9 & 7 & 7 & 83 & -68 & -18 & 8 & 12 & 7 \\
\hline 10 & 7 & 7 & 112 & 2.1. & 15 & 10 & 12 & 7 \\
\hline 11 & 7 & 7 & 88 & 31 & -22 & 11 & 12 & 7 \\
\hline 12 & 7 & 7 & 25 & 37 & -44 & -2 & 23 & 7 \\
\hline 13 & 7 & 7 & 38 & -3 & -34 & 0 & 13 & 7 \\
\hline-7 & 8 & 7 & 37 & 37 & -41 & 1. & 13 & 7 \\
\hline-6 & 8 & 7 & 25 & -43 & -48 & 2 & 13 & 7 \\
\hline-4 & 8 & 7 & 28 & -68 & -41 & 3 & 13 & 7 \\
\hline-3 & 8 & 7 & 58 & -60 & -23 & 4 & 13 & 7 \\
\hline-2 & 8 & 7 & 205 & -219 & 9 & 5 & 13 & 7 \\
\hline-1 & 8 & 7 & 221 & 211 & 7 & 6 & 13 & 7 \\
\hline 0 & 8 & 7 & 23 & 9 & -37 & 7 & 13 & 7 \\
\hline 1 & 8 & 7 & 31 & -49 & -32 & 8 & 33 & 7 \\
\hline 2 & 8 & 7 & 63 & $-I$ & -14 & 9 & 13 & 7 \\
\hline 3 & 8 & 7 & 126 & -205 & 8 & 10 & 13 & 7 \\
\hline 4 & 8 & 7 & 102 & 77 & 10 & 1 & 14 & 7 \\
\hline 5 & 8 & 7 & 72 & -43 & 14 & 2 & 24 & 7 \\
\hline 6 & 8 & 7 & 90 & 49 & 13 & 3 & 3.4 & 7 \\
\hline 7 & 8 & 7 & 151 & -246 & 9 & 4 & 24 & 7 \\
\hline 8 & 8 & 7 & 51 & 46 & -31 & 5 & 14 & 7 \\
\hline 9 & 8 & 7 & 209 & -91 & 14 & 6 & 14 & 7 \\
\hline 10 & 8 & 7 & 23 & 21 & -50 & 7 & 14 & 7 \\
\hline 11 & 8 & 7 & 74 & -18 & -20 & 9 & 14 & 7 \\
\hline 12 & 8 & 7 & 77 & 45 & -20 & $-5-$ & -12 & 8 \\
\hline 13 & 8 & 7 & 50 & 13 & -37 & -4 & -12 & 8 \\
\hline-6 & 9 & 7 & 112 & 122 & 17 & $-3-$ & -12 & 8 \\
\hline-5 & 9 & 7 & 158 & -146 & 12 & $-2-$ & -12 & 8 \\
\hline-4 & 9 & 7 & 223 & 233 & 15 & -3 & -12 & 8 \\
\hline-3 & 9 & 7 & 186 & -167 & 9 & & -12 & 8 \\
\hline-2 & 9 & 7 & 242 & 246 & 8 & & -12 & 8 \\
\hline-1 & 9 & 7 & 271 & -264 & 8 & $-7-$ & -1.1 & 8 \\
\hline 0 & 9 & 7 & 265 & 253 & 7 & $-6-$ & -11 & 8 \\
\hline 2 & 9 & 7 & 27 & -55 & -37 & $-5-$ & -11 & 8 \\
\hline 2 & 9 & 7 & 175 & 154 & 7 & $-4-$ & -11 & 8 \\
\hline 3 & 9 & 7 & 281 & -254 & 6 & $-3-$ & -11 & 8 \\
\hline 4 & 9 & 7 & 305 & 326 & 6 & $-2-$ & -11 & 8 \\
\hline 5 & 9 & 7 & $2 \pm 4$ & -108 & 10 & -1 & -11 & 8 \\
\hline 6 & 9 & 7 & 135 & 148 & 10 & & -12 & 8 \\
\hline 7 & 9 & 7 & 99 & -43 & 14 & & -21 & 8 \\
\hline 8 & 9 & 7 & 105 & 98 & 14 & & -11 & 8 \\
\hline 9 & 9 & 7 & 160 & -168 & 11 & & -11 & 8 \\
\hline 10 & 9 & 7 & 126 & 160 & 15 & -8 & -10 & 8 \\
\hline 11 & 9 & 7 & 23 & -104 & -52 & $-7-$ & -10 & 8 \\
\hline 12 & 9 & 7 & 68 & 18 & -29 & $-6-$ & -20 & 8 \\
\hline 13 & 9 & 7 & 3.2 & -11 & -50 & $-5-$ & -20 & 8 \\
\hline-5 & 10 & 7 & 52 & 39 & -26 & $-4-$ & -10 & $s$ \\
\hline-4 & 10 & 7 & 71 & -41 & -28 & $-3-$ & -10 & 8 \\
\hline-3 & 10 & 7 & 54 & -19 & -29 & -2 & -10 & 8 \\
\hline-2 & 10 & 7 & 80 & 5 & -23 & $-1-$ & -10 & 8 \\
\hline-1 & 10 & 7 & 68 & 1 & -26 & & -10 & 8 \\
\hline 0 & 10 & 7 & 104 & 3.20 & 14 & & -10 & 8 \\
\hline 1 & 10 & 7 & 83 & -87 & 15 & & -10 & 8 \\
\hline 2 & 10 & 7 & 34 & 92 & -35 & & -20 & 8 \\
\hline 3 & 10 & 7 & 25 & -4 & -32 & & -10 & 8 \\
\hline 4 & 10 & 7 & 45 & -39 & -29 & & -10 & 8 \\
\hline 5 & 20 & 7 & 46 & -38 & -29 & -9 & -9 & 8 \\
\hline 6 & 10 & 7 & 145 & 137 & 10 & -8 & -9 & 8 \\
\hline 7 & 20 & 7 & 43 & -28 & -33 & -7 & -9 & 8 \\
\hline 8 & 10 & 7 & 39 & 21 & -38 & -6 & -9 & 8 \\
\hline 9 & 10 & 7 & a4 & -21 & -24 & -5 & -9 & 8 \\
\hline 10 & 10 & 7 & 115 & 35 & 15 & -4 & -9 & 8 \\
\hline 11 & 10 & 7 & 47 & -37 & -38 & -3 & -9 & 8 \\
\hline-4 & 11 & 7 & 124 & 131 & 17 & -2 & -9 & 8 \\
\hline-3 & 21 & 7 & 105 & -92 & 26 & -1 & -9 & 8 \\
\hline-2 & 2.2 & 7 & 122 & 1.10 & 14 & 0 & -9 & 8 \\
\hline-1 & 11 & 7 & 174 & 178 & 20 & 2 & -9 & 8 \\
\hline & 37 & 7 & 232 & 38 & & 3 & -9 & 8 \\
\hline
\end{tabular}

is $\mathrm{k} 1 \mathrm{~K}$ 1OFO $10 \mathrm{FC}$ los

$$
\begin{array}{llllll}
4 & -9 & 8 & 52 & 6 & -31
\end{array}
$$

$\begin{array}{llllll}4 & -9 & 8 & 52 & 6 & -31 \\ 5 & -9 & 8 & 60 & 39 & -31\end{array}$

$\begin{array}{llllll}5 & -9 & 8 & 76 & 38 & -22\end{array}$

$\begin{array}{rrrrrr}-10 & -8 & 8 & 14 & 19 & -54 \\ -9 & -8 & 2 & 86 & -39 & -17\end{array}$

$\begin{array}{rrrrrr}-8 & -8 & 8 & 45 & 38 & -33 \\ -7 & -8 & 8 & 65 & -101 & -22\end{array}$

$\begin{array}{llllll}-6 & -8 & 8 & 127 & 131 & 12\end{array}$

$\begin{array}{llllll}-5 & -8 & 8 & 105 & -113 & 14\end{array}$

$\begin{array}{rrrrrr}-4 & -8 & 8 & 85 & 78 & 16 \\ -3 & -8 & 8 & 86 & -63 & 34\end{array}$

$\begin{array}{llllll}-2 & -8 & 8 & 28 & 54 & -38\end{array}$

$\begin{array}{lllllll}-1 & -8 & 8 & 206 & -185 & 8\end{array}$

$\begin{array}{lllll}0 & -8 & 8 & 278 & 283\end{array}$

$\begin{array}{rrrrr}1 & -8 & 8 & 300 & -313 \\ 2 & -8 & 8 & 284 & 303\end{array}$

$\begin{array}{rrrrrr}3 & -8 & 8 & 219 & -224 & 8\end{array}$

$\begin{array}{rrrrrr}4 & -8 & 8 & 104 & 101 & 14 \\ 5 & -8 & 8 & 137 & -150 & 14\end{array}$

$\begin{array}{llllll}6 & -8 & 8 & 148 & 175 & 14\end{array}$

$\begin{array}{llllll}7 & -8 & 2 & 37 & -56 & -43\end{array}$

$\begin{array}{rrrrrr}-10 & -7 & 8 & 48 & 10 & -36 \\ -9 & -7 & 8 & 39 & -34 & -37\end{array}$

$\begin{array}{rrrrrr}-8 & -7 & 8 & 54 & 49 & -30\end{array}$

$\begin{array}{llllll}-7 & -7 & 8 & 109 & -58 & 13\end{array}$

$\begin{array}{rrrrrr}-6 & -7 & 8 & 48 & 73 & -26\end{array}$

$\begin{array}{rrrrrr}-5 & -7 & 8 & 73 & -84 & -17 \\ -4 & -7 & 8 & 43 & 37 & -29\end{array}$

$\begin{array}{llllll}-3 & -7 & 8 & 113 & -97 & 11\end{array}$

$\begin{array}{llllll}-2 & -7 & 8 & 69 & -45 & -14\end{array}$

$\begin{array}{llllll}-1 & -7 & 8 & 59 & 35 & -36\end{array}$

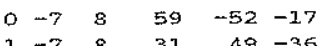

$\begin{array}{llllll}1 & -7 & 8 & 31 & 49 & -36 \\ 2 & -7 & 8 & 50 & 59 & -28\end{array}$

$\begin{array}{llllll}3 & -7 & 8 & 94 & -89 & 15\end{array}$

$\begin{array}{llllll}4 & -7 & 8 & 127 & 119 & 12\end{array}$

$\begin{array}{llllll}5 & -7 & 8 & 132 & -136 & 13\end{array}$

$\begin{array}{rrrrrr}6 & -7 & 8 & 48 & -19 & -32 \\ 7 & -7 & 8 & 31 & 16 & -45\end{array}$

$\begin{array}{llllll}7 & -7 & 8 & 31 & 16 & -45 \\ 8 & -7 & 8 & 53 & 24 & -35\end{array}$

$\begin{array}{llllll}-10 & -6 & 8 & 72 & 32 & -21\end{array}$

$\begin{array}{rrrrrr}-9 & -6 & 8 & 29 & -38 & -44 \\ -8 & -6 & 8 & 31 & 24 & -32\end{array}$

$\begin{array}{rrrrrr}-8 & -6 & 8 & 31 & 24 & -32 \\ -7 & -6 & 8 & 61 & -80 & -26\end{array}$

$\begin{array}{lllllll}-6 & -6 & 8 & 228 & 223 & 8\end{array}$

$\begin{array}{lllll}-5 & -6 & 8 & 212 & -209\end{array}$

$\begin{array}{lllll}-4 & -6 & 8 & 242 & 252\end{array}$

$\begin{array}{lllll}-3 & -6 & 8 & 253 & -254\end{array}$

$\begin{array}{rrrrr}-2 & -6 & 8 & 157 & 167 \\ -1 & -6 & 8 & 335 & -322\end{array}$

$\begin{array}{lllll}0 & -6 & 8 & 238 & 233\end{array}$

$\begin{array}{llllll}3 . & -6 & 8 & 196 & -192 & 7\end{array}$

$\begin{array}{rrrrrr}2 & -6 & 8 & 209 & 201 & 7\end{array}$

$\begin{array}{rrrrrr}3 & -6 & 8 & 142 & -144 & 10 \\ 4 & -6 & 8 & 220 & 221 & 8\end{array}$

$\begin{array}{llllll}5 & -6 & 8 & 128 & -106 & 10\end{array}$

$\begin{array}{llllll}6 & -6 & 8 & 191 & 205 & 10\end{array}$

$\begin{array}{llllll}7 & -6 & 8 & 163 & -189 & 32\end{array}$

$\begin{array}{rrrrrr}8 & -6 & 8 & 93 & 84 & 18 \\ 9 & -6 & 8 & 77 & -65 & -21\end{array}$

$\begin{array}{llllll}-11 & -5 & 8 & 54 & 8 & -28\end{array}$

$\begin{array}{llllll}-10 & -5 & 8 & 70 & 1 & -20\end{array}$

$\begin{array}{llllll}-9 & -5 & 8 & 12 & -10 & -49 \\ -8 & -5 & 8 & 12 & -40 & -48\end{array}$

$\begin{array}{lllllr}-7 & -5 & 8 & 80 & -44 & 36\end{array}$

$\begin{array}{rrrrrr}-6 & -5 & 8 & 11 & 101 & -43 \\ -5 & -5 & 8 & 119 & -138 & 10\end{array}$

$\begin{array}{rrrrrr}-5 & -5 & 8 & 119 & -138 & 10 \\ -4 & -5 & 8 & 183 & 186 & 7\end{array}$

$\begin{array}{rrrrrr}-3 & -5 & 8 & 89 & -79 & 11 \\ -2 & -5 & 8 & 35 & 35 & -27\end{array}$

$\begin{array}{rrrrrr}-1 & -5 & 8 & 98 & -104 & 10\end{array}$

$\begin{array}{llllll}0 & -5 & 8 & 166 & 164 & 7\end{array}$

$\begin{array}{rrrrrr}1 & -5 & 8 & 198 & -215 & 7 \\ 2 & -5 & 8 & 96 & 78 & 11\end{array}$

$\begin{array}{rrrrrr}2 & -5 & 8 & 96 & 78 & 11 \\ 3 & -5 & 8 & 58 & 51 & -17\end{array}$

$\begin{array}{rrrrrr}4 & -5 & 8 & 148 & -164 & 9\end{array}$

$\begin{array}{rrrrrr}5 & -5 & 8 & 36 & 0 & -33 \\ 6 & -5 & 8 & 179 & 158 & 10\end{array}$

$\begin{array}{rrrrrr}6 & -5 & 8 & 179 & 158 & 10 \\ 7 & -5 & 8 & 13 & 41 & -51\end{array}$

$\begin{array}{llllll}8 & -5 & 8 & 45 & -22 & -32\end{array}$

$\begin{array}{rlllll}9 & -5 & 8 & 44 & -20 & -30 \\ 10 & -5 & 8 & 85 & -12 & -18\end{array}$

$\begin{array}{rrrrrr}10 & -5 & 8 & 85 & -12 & -1.8 \\ -11 & -4 & 8 & 53 & -75 & -35\end{array}$

$\begin{array}{llllll}-10 & -4 & 8 & 78 & 49 & -19\end{array}$

$\begin{array}{rrrrrr}-9 & -4 & 8 & 87 & -49 & 16 \\ -8 & -4 & 8 & 74 & 56 & -17\end{array}$

$\begin{array}{rrrrrr}-8 & -4 & 8 & 74 & 56 & -17 \\ -7 & -4 & 8 & 88 & -102 & 17\end{array}$

$\begin{array}{llllll}-6 & -4 & 8 & 115 & 85 & 11\end{array}$

$\begin{array}{rrrrrr}-5 & -4 & 8 & 109 & -131 & 10 \\ -4 & -4 & 8 & 158 & 160 & 7\end{array}$ $\mathrm{h} \mathrm{k} I \mathrm{HOFO}$ 1OFC $10 \mathrm{~S}$

$\mathrm{h} k 1$ 1OFO 1OFC $10 \mathrm{~S}$ $\begin{array}{llllll}-5 & 0 & 8 & 345 & -349 & 7\end{array}$

$\begin{array}{llllll}-3 & 0 & 8 & 288 & -306 & 4\end{array}$

$\begin{array}{lllll}-2 & 0 & 8 & 267 & 282\end{array}$

$\begin{array}{llllll}0 & 0 & 8 & 87 & 126 & 8\end{array}$

$2008373 \quad 410 \quad 11$

$\begin{array}{llllll}3 & 0 & 8 & 385 & -390 & 4\end{array}$

$\begin{array}{llllll}4 & 0 & 8 & 855 & 869 & 22\end{array}$

$\begin{array}{rrrrrr}5 & 0 & 8 & 431 & -448 & 4 \\ 6 & 0 & 8 & 392 & 403 & 12\end{array}$

$\begin{array}{lllll}7 & 0 & 8 & 195 & -209\end{array}$

$808154159 \quad 6$

$\begin{array}{llllll}10 & 0 & 8 & 131 & 135 & 18\end{array}$

$\begin{array}{llllll}1.1 & 0 & 8 & 47 & -35 & -21\end{array}$

$12 \circ 8 \quad 104 \quad 41 \quad 16$

$\begin{array}{rlllll}13 & 0 & 8 & 34 & -27 & -32 \\ -10 & 1 & 8 & 81 & -52 & -17\end{array}$

$\begin{array}{llllll}-9 & 1 & 8 & 62 & -2 & -27\end{array}$

$63-35-20$

$\begin{array}{lllll}-3 & 8 & 28 & -33 & -30\end{array}$

$\begin{array}{llllll}-1 & -3 & 8 & 54 & -12 & -18\end{array}$

$\begin{array}{llllll}0 & -3 & 8 & 39 & 72 & -18 \\ 1 & -3 & 8 & 54 & 14 & -19\end{array}$

$\begin{array}{llllll}2 & -3 & 8 & 40 & -58 & -24\end{array}$

$\begin{array}{llllll}3 & -3 & 8 & 48 & 41 & -22\end{array}$

$\begin{array}{llllll}4 & -3 & 8 & 87 & -23 & 11\end{array}$

$\begin{array}{rrrrrr}5 & -3 & 8 & 67 & 7 & -15 \\ 6 & -3 & 8 & 188 & 190 & 8\end{array}$

$\begin{array}{rrrrrr}7 & -3 & 8 & 188 & 190 & 8 \\ 7 & -3 & 8 & 79 & -23 & -1.7\end{array}$

$\begin{array}{llllll}8 & -3 & 8 & 96 & -108 & 15\end{array}$

$\begin{array}{llllll}9 & -3 & 8 & 67 & 35 & -19\end{array}$

$\begin{array}{llllll}10 & -3 & 8 & 60 & -9 & -29\end{array}$

$\begin{array}{rrrrrr}11 & -3 & 8 & 42 & 25 & -41 \\ -11 & -2 & 8 & 67 & 7 & -30\end{array}$

$-10-2 \quad 8 \quad 40 \quad 55-41$

$\begin{array}{llllll}-9 & -2 & 8 & 46 & -43 & -32\end{array}$

$\begin{array}{rrrrrr}-8 & -2 & 8 & 100 & 67 & 14 \\ -7 & -2 & 8 & 161 & -161 & 10\end{array}$

$\begin{array}{lllll}-7 & -2 & 8 & 161 & -161 \\ -6 & -2 & 8 & 299 & 340\end{array}$

$\begin{array}{lllll}-5 & -2 & 8 & 254 & -261\end{array}$

$\begin{array}{lllll}-4 & -2 & 8 & 152 & 166\end{array}$

$\begin{array}{lllll}-3 & -2 & 8 & 282 & -299\end{array}$

$\begin{array}{lllll}-2 & -2 & 8 & 284 & 317\end{array}$

$\begin{array}{rrrrr}-1 & -2 & 8 & 614 & -619 \\ 0 & -2 & 8 & 499 & 544\end{array}$

$\begin{array}{lllll}1 & -2 & 8 & 500 & -529\end{array}$

$\begin{array}{lllll}2 & -2 & 8 & 255 & 271\end{array}$

$\begin{array}{lllll}3 & -2 & 8 & 282 & -300\end{array}$

$\begin{array}{rrrrr}4 & -2 & 8 & 243 & 238 \\ 5 & -2 & 8 & 202 & -202\end{array}$

$\begin{array}{lllll}6 & -2 & 8 & 252 & 252\end{array}$

$\begin{array}{llllll}7 & -2 & 8 & 104 & -95 & 12\end{array}$

$\begin{array}{llllll}8 & -2 & 8 & 144 & 128 & 11\end{array}$

$\begin{array}{rrrrrr}9 & -2 & 8 & 122 & -128 & 33 \\ 10 & -2 & 8 & 80 & 56 & -18\end{array}$

$\begin{array}{llllll}11 & -2 & 8 & 31 & -35 & -44\end{array}$

$\begin{array}{llllll}12 & -2 & 8 & 42 & 21 & -37\end{array}$

$\begin{array}{rrrrrr}-10 & -1 & 8 & 83 & 0 & -24 \\ -9 & -1 & 8 & 60 & 7 & -20\end{array}$

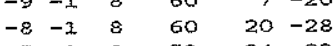

$\begin{array}{llllll}-7 & -1 & 8 & 53 & -24 & -23 \\ -6 & -1 & 8 & 56 & 0 & -79\end{array}$

$\begin{array}{lllll}-5 & -1 & 8 & 101 & 86 \\ 10\end{array}$

$\begin{array}{rrrrrr}-4 & -1 & 8 & 68 & -13 & 12 \\ -3 & -1 & 8 & 50 & -45 & -15\end{array}$

$\begin{array}{rrrrrr}-3 & -1 & 8 & 50 & -45 & -15 \\ -2 & -1 & 8 & 137 & -125 & 7\end{array}$

$\begin{array}{llllll}-1 . & -1 & 8 & 187 & 191 & 6\end{array}$

$\begin{array}{llllll}0 & -1 & 8 & 194 & 38 & -8\end{array}$

$\begin{array}{rrrrr}1 & -1 & 8 & 184 & -188 \\ 2 & -1 & 8 & 407 & 387\end{array}$

$\begin{array}{rrrrr}2 & -1 & 8 & 407 & 387 \\ 3 & -1 & 8 & 314 & -302\end{array}$

$\begin{array}{llllll}4 & -1 & 8 & 87 & 56 & 10\end{array}$

$\begin{array}{llllll}5 & -1 & 8 & 108 & 89 & 10\end{array}$

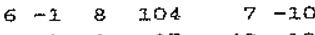

$\begin{array}{rrrrrr}7 & -1 & 8 & 87 & 42 & 13 \\ 8 & -1 & 8 & 89 & -72 & 16\end{array}$

$9-1 \quad 8 \quad 122 \quad 49 \quad 12$

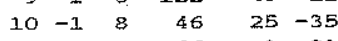

$\begin{array}{llllll}11 & -1 & 8 & 66 & 1 & -21\end{array}$

$\begin{array}{llllll}12 & -1 & 8 & 53 & 24 & -34\end{array}$

$\begin{array}{rrrrrr}-9 & 0 & 8 & 36 & 14 & -26 \\ -9 & 0 & 8 & 80 & -73 & 13\end{array}$

$\begin{array}{llllrr}-8 & 0 & 8 & 44 & 39 & -24 \\ -7 & 0 & 8 & 134 & -106\end{array}$

$\begin{array}{rrrrrr}-7 & 0 & 8 & 134 & -106 & 8 \\ -6 & 0 & 8 & 162 & 157 & 7\end{array}$
$12-59-48$

$\begin{array}{rrr}80 & 38 & 13 \\ 59 & -14 & -23\end{array}$

$\begin{array}{rrrrrr}-5 & 1 & 8 & 59 & -14 & -23 \\ -4 & 1 & 8 & 264 & -278 & 6\end{array}$

$\begin{array}{ll}-7 & 1 \\ -6 & 1\end{array}$

$\begin{array}{rrrrrr}-3 & 1 & 8 & 4,1 & 46 & -18 \\ -2 & 1 & 8 & 18 & -12 & -33\end{array}$

$\begin{array}{rrrrrr}-1 & 1 & 8 & 18 & -12 & -33 \\ -1 & 1 & 8 & 411 & 371 & \end{array}$

$\begin{array}{llllll}0 & 1 & 8 & 57 & -83 & -14\end{array}$

$1 \quad 1 \quad 8 \quad 167138$

$\begin{array}{llllr}3 & 1 & 8 & 332 & 340\end{array}$

$\begin{array}{llllll}4 & 1 & 8 & 130 & -39 & -7\end{array}$

$18206 \quad 1846$

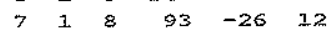

$\begin{array}{llllll}8 & 1 & 8 & 73 & -39 & -16\end{array}$

$\begin{array}{rrrrrr}9 & 1 & 8 & 83 & 8 & -18 \\ 10 & 1 & 8 & 96 & 38 & 16\end{array}$

$\begin{array}{llllll}11 & 1 & 8 & 90 & -15 & 16\end{array}$

$\begin{array}{llllll}12 & 1 & 8 & 36 & 28 & -41\end{array}$

$\begin{array}{llllll}13 & 1 & 8 & 65 & -7 & -28\end{array}$

$\begin{array}{rrrrrr}10 & 2 & 8 & 32 & 9 & -41 \\ -9 & 2 & 8 & 60 & -25 & -29\end{array}$

$\begin{array}{llllll}-8 & 2 & 8 & 47 & 57 & -35\end{array}$

$\begin{array}{llllll}-7 & 2 & 8 & 135 & -106 & 14\end{array}$

$\begin{array}{rrrrrr}-6 & 2 & 8 & 179 & 189 & 9 \\ -5 & 2 & 8 & 268 & -255 & 6\end{array}$

$\begin{array}{rrrrr}-5 & 2 & 8 & 268 & -255 \\ -4 & 2 & 8 & 237 & 225\end{array}$

$\begin{array}{llllll}-3 & 2 & 8 & 527 & -530\end{array}$

$\begin{array}{llllll}-2 & 2 & 8 & 338 & 327 & 5\end{array}$

$\begin{array}{rrrrrr}-1 & 2 & 8 & 323 & -338 & 5 \\ 0 & 2 & 8 & 610 & 688 & 5\end{array}$

$\begin{array}{rrrrrr}1 & 2 & 8 & 610 & 688 & 5 \\ 1 & 2 & 8 & 174 & -191 & 6\end{array}$

$228608665 \quad 5$

$\begin{array}{rrrrrr}3 & 2 & 8 & 483 & -514 & 5 \\ 4 & 2 & 8 & 338 & 337 & 5\end{array}$

$528 \quad 347-347 \quad 5$

$\begin{array}{rrrrrr}7 & 2 & 8 & 160 & -154 & 8\end{array}$

$8 \quad 2 \quad 8 \quad 87 \quad 109 \quad 33$

$\begin{array}{llllll}9 & 2 & 8 & 166 & -173 & 10\end{array}$

1028 118 $150 \quad 14$

$\begin{array}{rrrrrr}11 & 2 & 8 & 92 & -99 & 16 \\ 12 & 2 & 8 & 79 & 60 & -18\end{array}$

$\begin{array}{llllll}33 & 2 & 8 & 52 & -28 & -27\end{array}$

$\begin{array}{llllll}-9 & 3 & 8 & 12 & 24 & -49\end{array}$

$\begin{array}{llllll}-8 & 3 & 8 & 51 & -43 & -31\end{array}$

$\begin{array}{rrrrrr}-7 & 3 & 8 & 80 & 55 & -19 \\ -6 & 3 & 8 & 135 & 138 & 10\end{array}$

$\begin{array}{llllll}-3 & 3 & 8 & 80 & 45 & 12\end{array}$

$\begin{array}{llllll}-2 & 3 & 8 & 100 & -133 & 9\end{array}$

$\begin{array}{rrrrrr}-1 & 3 & 8 & 269 & 154 & 6 \\ 0 & 3 & 8 & 52 & -127 & -15\end{array}$

$\begin{array}{llllll}1 & 3 & 8 & 242 & 239 & 5\end{array}$

$\begin{array}{llllll}2 & 3 & 8 & 169 & -166 & 6\end{array}$

$\begin{array}{rrrrrr}3 & 3 & 8 & 260 & 252 & 5 \\ 4 & 3 & 8 & 122 & 63 & 8\end{array}$

$\begin{array}{llllll}4 & 3 & 8 & 122 & 63 & 8 \\ 5 & 3 & 8 & 101 & 52 & 9\end{array}$

$\begin{array}{rrrrrr}6 & 3 & 8 & 53 & -73 & -18 \\ 7 & 3 & 8 & 186 & 128 & 7\end{array}$

$\begin{array}{llllll}8 & 3 & 8 & 49 & -59 & -19\end{array}$

$\begin{array}{rrrrrr}9 & 3 & 8 & 93 & -38 & 1.3 \\ 20 & 3 & 8 & 27 & 22 & -45\end{array}$

$\begin{array}{rrrrrr}11 & 3 & 8 & 29 & 12 & -42 \\ 12 & 3 & 8 & 39 & 0 & -38\end{array}$

$\begin{array}{llllll}13 & 3 & 8 & 85 & -3 & -19\end{array}$ $\begin{array}{llllll}-5 & 3 & 8 & 59 & -14 & -25\end{array}$ 


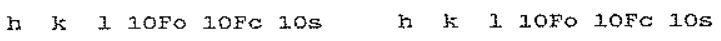

\begin{tabular}{|c|c|c|c|c|c|c|c|c|}
\hline-9 & 4 & 8 & 97 & -100 & 18 & -4 & 8 & 8 \\
\hline-8 & A & 8 & 95 & 89 & 17 & -3 & 8 & 8 \\
\hline-7 & 4 & 8 & 321 & -108 & 14 & -2 & 8 & 8 \\
\hline-6 & 4 & 8 & 171 & 173 & 10 & -1 & 8 & 8 \\
\hline-5 & 4 & 8 & 180 & -181 & 9 & 0 & 8 & $s$ \\
\hline-4 & 4 & 8 & 401 & 415 & 6 & $I$ & 8 & 8 \\
\hline-3 & 4 & 8 & 573 & -572 & 7 & 2 & 8 & \& \\
\hline-2 & $\underline{2}$ & 8 & 604 & 580 & 6 & 3 & 8 & 8 \\
\hline-1 & 4 & 8 & 427 & -459 & 6 & 4 & 8 & 8 \\
\hline 0 & 4 & 8 & 610 & 614 & 5 & 5 & 8 & 8 \\
\hline 1 & 4 & 8 & 496 & -505 & 5 & 6 & 8 & $B$ \\
\hline 2 & 4 & 8 & 486 & 467 & 5 & 7 & 8 & 8 \\
\hline 3 & 4 & 8 & 509 & -517 & 5 & 8 & 8 & 8 \\
\hline 4 & 1 & 8 & 407 & 429 & 6 & 9 & 8 & 8 \\
\hline 5 & 4 & 8 & 378 & -364 & 5 & 10 & 8 & 8 \\
\hline 6 & 4 & 8 & 364 & 382 & 6 & 11 & 8 & 8 \\
\hline 7 & 4 & 8 & $3 \pm 9$ & -317 & 6 & 12 & 8 & 8 \\
\hline 8 & 4 & 8 & 67 & 71 & -17 & 13 & 8 & 8 \\
\hline 9 & 4 & 8 & 117 & -62 & 31 & -6 & 9 & 8 \\
\hline 10 & 4 & 8 & 41 & -3 & -32 & -5 & 9 & 8 \\
\hline 21 & 4 & 8 & 54 & -76 & -31 & -4 & 9 & 8 \\
\hline 12 & $\therefore$ & 8 & 49 & 62 & -35 & -3 & 9 & 8 \\
\hline 13 & 4 & 8 & 11 & -40 & -44 & -2 & 9 & 8 \\
\hline-9 & 5 & 8 & 71 & -28 & -21 & -1 & 9 & 8 \\
\hline-8 & 5 & 8 & 50 & -24 & -34 & 0 & 9 & 8 \\
\hline-7 & 5 & 8 & 57 & -4 & -23 & 1 & 9 & 8 \\
\hline-6 & 5 & 8 & 109 & 35 & 12 & 2 & 9 & 8 \\
\hline-5 & 5 & 8 & 21 & -25 & -42 & 3 & 9 & 8 \\
\hline-4 & $s$ & 8 & 261 & -154 & 8 & 4 & 9 & 8 \\
\hline-3 & 5 & 8 & III & 86 & 11 & 5 & 9 & 8 \\
\hline-2 & 5 & 8 & 57 & -7 & -16 & 6 & 9 & 8 \\
\hline-1 & 5 & 8 & 54 & 9 & -15 & 7 & 9 & 8 \\
\hline 0 & 5 & 8 & 174 & 162 & 6 & 8 & 9 & 8 \\
\hline 1 & 5 & 8 & 93 & 59 & 9 & 9 & 9 & 8 \\
\hline 2 & 5 & 8 & 32 & -1 & -25 & 10 & 9 & 8 \\
\hline 3 & 5 & 8 & 142 & -110 & 7 & 11 & 9 & 8 \\
\hline 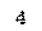 & 5 & 8 & 273 & 296 & 5 & 3.2 & 9 & 8 \\
\hline 5 & 5 & 8 & 123 & -84 & 8 & 13 & 9 & 8 \\
\hline 6 & 5 & 8 & 252 & 119 & 8 & -5 & 10 & 8 \\
\hline 7 & $\mathrm{~s}$ & 8 & 274 & 256 & 8 & -4 & 10 & 8 \\
\hline 8 & 5 & 8 & 102 & -56 & 12 & -3 & 10 & 8 \\
\hline 9 & 5 & 3 & 79 & 36 & -3.8 & -2 & 10 & 8 \\
\hline 10 & 5 & 8 & 108 & 86 & 14 & -1 & 10 & 8 \\
\hline 11 & 5 & 8 & 86 & 43 & 17 & 0 & 10 & 8 \\
\hline 12 & 5 & 8 & 65 & -12 & -31 & $I$ & 30 & 8 \\
\hline 13 & 5 & 8 & 58 & 5 & -32 & 2 & 10 & 8 \\
\hline-8 & 6 & s & 69 & 84 & -29 & 3 & 20 & 8 \\
\hline-7 & 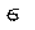 & 8 & 127 & -152 & 15 & 4 & 10 & 8 \\
\hline-6 & 6 & 8 & 185 & 191 & 10 & 5 & 10 & 8 \\
\hline-5 & 6 & 8 & 205 & -209 & 9 & 6 & 10 & 8 \\
\hline-4 & 5 & 8 & 175 & 172 & 9 & 7 & 10 & 8 \\
\hline-3 & 6 & 8 & 444 & -786 & 7 & 8 & 10 & 8 \\
\hline-2 & 5 & 8 & 346 & 321 & $\epsilon$ & 9 & 10 & 8 \\
\hline-1 & 6 & s & 401 & -386 & 6 & 10 & 10 & 8 \\
\hline 0 & 6 & 8 & 407 & 402 & 6 & 11 & 10 & 8 \\
\hline 1 & 6 & 8 & 327 & -322 & 5 & 12 & 10 & 8 \\
\hline 2 & 6 & 8 & 225 & 240 & 5 & -4 & 11 & 8 \\
\hline 3 & 6 & 8 & 225 & -214 & 5 & -3 & 1.1 & 8 \\
\hline$a$ & 6 & 8 & 286 & 209 & 6 & -2 & 11 & 8 \\
\hline 5 & 6 & 8 & 323 & -333 & 5 & -3 & 13 & 8 \\
\hline 6 & 6 & 8 & 383 & 371 & 5 & 0 & 21 & 8 \\
\hline 7 & 6 & 8 & 330 & -357 & 6 & 1 & $2 I$ & 8 \\
\hline 8 & 6 & 8 & 171 & 152 & 9 & 2 & 22 & 8 \\
\hline 9 & 6 & 8 & 198 & -219 & 10 & 3 & 21 & 8 \\
\hline 10 & 6 & 8 & 89 & 74 & 16 & 4 & 21 & 8 \\
\hline 11. & 6 & 3 & 23 & 28 & -42 & 5 & 11 & 3 \\
\hline 23 & 6 & 8 & 60 & 10 & -25 & 6 & 13 & 8 \\
\hline-7 & 7 & 8 & 49 & 67 & -29 & 7 & 11 & 8 \\
\hline-6 & 7 & 8 & 38 & 3 & -39 & 8 & 11 & 8 \\
\hline-5 & 7 & 8 & 28 & -53 & -40 & 9 & 3.1 & a \\
\hline-4 & 7 & 8 & 89 & 46 & 14 & 10 & 11 & 8 \\
\hline-3 & 7 & 8 & 48 & 2 & -27 & 11 & 11 & 8 \\
\hline-2 & 7 & 8 & 103 & -102 & 11 & 12 & 11 & 8 \\
\hline-1 & 7 & 8 & 67 & 1 & -15 & -2 & 12 & 8 \\
\hline 0 & 7 & 8 & 119 & 126 & 10 & $-\lambda$ & 12 & 8 \\
\hline 1 & 7 & 8 & 145 & -135 & 8 & 0 & 12 & 8 \\
\hline 2 & 7 & 8 & 230 & 138 & 8 & 1 & 12 & 8 \\
\hline 3 & 7 & 8 & 68 & 2 & 12 & 2 & 12 & 8 \\
\hline 4 & 7 & 8 & 110 & 99 & 8 & 3 & 12 & 8 \\
\hline 5 & 7 & 8 & 88 & 51 & 1.1 & 4 & 12 & 8 \\
\hline 6 & 7 & 8 & 92 & 101 & 12 & 5 & 12 & 8 \\
\hline 7 & 7 & 8 & 148 & 79 & 9 & 6 & 12 & 8 \\
\hline 8 & 7 & 8 & 61 & -18 & -28 & 7 & 22 & 8 \\
\hline 9 & 7 & 8 & 54 & 6 & -31 & 8 & 12 & 8 \\
\hline 1.0 & 7 & 8 & 102 & -4 & 16 & 9 & 12 & 8 \\
\hline 12 & 7 & 8 & 76 & 45 & -19 & 10 & 12 & 8 \\
\hline 13 & 7 & 8 & 52 & 2 & -36 & -3 & 13 & 8 \\
\hline-7 & 8 & 8 & 75 & -54 & -29 & 0 & 13 & 8 \\
\hline-6 & 8 & 8 & 174 & 131 & 15 & 1 & 13 & 8 \\
\hline-5 & 8 & 8 & 1.14 & -101 & 13 & 2 & 13 & 8 \\
\hline
\end{tabular}

\begin{abstract}
h $\mathrm{k}$ l 1OFO $10 \mathrm{FC}$ 10s
\end{abstract}

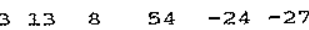

$\begin{array}{llllll}4 & 13 & 8 & 54 & -24 & -27\end{array}$

$\begin{array}{llllll}5 & 13 & 8 & 93 & -93 & 16\end{array}$

$\begin{array}{llllll}6 & 13 & 8 & 115 & 113 & 15\end{array}$

$\begin{array}{llllll}713 & 8 & 114 & -93 & 16\end{array}$

$\begin{array}{rrrrrr}8 & 13 & 8 & 68 & 31 & -30 \\ 9 & 13 & 8 & 75 & -11 & -29\end{array}$

$\begin{array}{rrrrrr}9 & 13 & 8 & 75 & -11 & -29 \\ 10 & 13 & 8 & 53 & 0 & -35\end{array}$

$\begin{array}{llllll}1 & 14 & 8 & 137 & -131 & 14\end{array}$

$214 \quad 8 \quad 205 \quad 116 \quad 16$

$\begin{array}{rrrrrr}3 & 14 & 8 & 67 & -88 & -29 \\ 4 & 14 & 8 & 92 & 103 & 17\end{array}$

$\begin{array}{llllll}5 & 14 & 8 & 78 & -65 & -17\end{array}$

$\begin{array}{llllll}6 & 14 & 8 & 38 & 78 & -34\end{array}$

$\begin{array}{llllll}714 & 8 & 67 & -97 & -25\end{array}$

$\begin{array}{llllll}8 & 14 & 8 & 61 & 116 & -36\end{array}$

$\begin{array}{rrrrr}-4-12 & 9 & 61 & 63 & -25 \\ -3-12 & 9 & 51 & -49 & -28\end{array}$

$\begin{array}{lllll}-3-12 & 9 & 51 & -49 & -28 \\ -2-12 & 9 & 47 & 36 & -38\end{array}$

$\begin{array}{lllll}-1-12 & 9 & 60 & -64 & -31\end{array}$

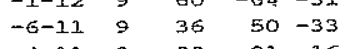

$\begin{array}{rrrrr}-4-11 & 9 & 88 & 91 & 16 \\ -3-11 & 9 & 114 & -98 & 13\end{array}$

$\begin{array}{lllll}-2-11 & 9 & 109 & 95 & 15\end{array}$

$\begin{array}{lllll}-1-11 & 9 & 34 & -96 & -42\end{array}$

$0-1199115 \quad 133 \quad 16$

$\begin{array}{rrrrr}1-21 & 9 & 55 & -147 & -30 \\ 2-11 & 9 & 220 & 107 & 15\end{array}$

3-11 $993-108 \quad 18$

$-8-10 \quad 9 \quad 66 \quad 56-31$

$\begin{array}{rrrrr}-8-10 & 9 & 66 & 56 & -31 \\ -7-10 & 9 & 102 & -57 & 16\end{array}$

$\begin{array}{rrrrr}-6-10 & 9 & 109 & 66 & 14 \\ -5-10 & 9 & 58 & -54 & -23\end{array}$

$\begin{array}{rrrrr}-5-10 & 9 & 58 & -54 & -23 \\ -4-10 & 9 & 36 & 56 & -36\end{array}$

$\begin{array}{lllll}-3-10 & 9 & 57 & -72 & -19\end{array}$

$\begin{array}{lllll}-2-10 & 9 & 59 & 63 & -27\end{array}$

$\begin{array}{ccccc}-1-10 & 9 & 43 & -20 & -32 \\ 0-10 & 9 & 33 & 37 & -37\end{array}$

$\begin{array}{lllll}1-20 & 9 & 48 & -4 & -32\end{array}$

$\begin{array}{lllll}2-10 & 9 & 30 & 38 & -36\end{array}$

$\begin{array}{rrrrr}3-10 & 9 & 54 & -84 & -36 \\ 4-10 & 9 & 49 & 17 & -36\end{array}$

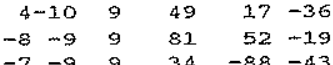

$\begin{array}{lllllr}-7 & -9 & 9 & 34 & -88 & -43 \\ -6 & -9 & 9 & 95 & 115 & 16\end{array}$

$\begin{array}{llllll}-5 & -9 & 9 & 121 & -135 & 13\end{array}$

$\begin{array}{llllll}-4 & -9 & 9 & 163 & 189 & 10\end{array}$

$\begin{array}{llllll}-3 & -9 & 9 & 177 & -191 & 9\end{array}$

$\begin{array}{rrrrrr}-2 & -9 & 9 & 76 & 111 & -19 \\ -1 & -9 & 9 & 139 & -130 & 11\end{array}$

$\begin{array}{llllll}0 & -9 & 9 & 181 & 184 & 10\end{array}$

$\begin{array}{llllll}1 & -9 & 9 & 162 & -166 & 13\end{array}$

$\begin{array}{rrrrrr}2 & -9 & 9 & 155 & 163 & 12 \\ 3 & -9 & 9 & 73 & -77 & -20\end{array}$

$\begin{array}{rrrrrr}3 & -9 & 9 & 73 & -77 & -20 \\ 4 & -9 & 9 & 31 & 69 & -45\end{array}$

$\begin{array}{rrrrrr}4 & -9 & 9 & 118 & -152 & 17\end{array}$

$\begin{array}{rrrrrr}6 & -9 & 9 & 98 & 231 & -20 \\ -9 & -8 & 9 & 25 & -10 & -48\end{array}$

$\begin{array}{rrrrrr}-9 & -8 & 9 & 25 & -10 & -48 \\ -8 & -8 & 9 & 57 & 26 & -24\end{array}$

$\begin{array}{llllll}-7 & -8 & 9 & 62 & -35 & -22\end{array}$

$\begin{array}{llllll}-6 & -8 & 9 & 59 & 47 & -28\end{array}$

$\begin{array}{rrrrrr}-5 & -8 & 9 & 79 & -68 & 16 \\ -4 & -8 & 9 & 86 & 81 & 15\end{array}$

$\begin{array}{rrrrrr}-4 & -8 & 9 & 86 & 81 & 15 \\ -3 & -8 & 9 & 67 & -11 & -1.7\end{array}$

$\begin{array}{rrrrrr}-2 & -8 & 9 & 11 & 12 & -43 \\ -1 & -8 & 9 & 76 & -92 & -17\end{array}$

$\begin{array}{llllll}0 & -8 & 9 & 74 & 100 & -17\end{array}$

$\begin{array}{llllll}1 . & -8 & 9 & 101 & -106 & 14\end{array}$

$\begin{array}{rrrrrr}2 & -8 & 9 & 46 & 94 & -34 \\ 3 & -8 & 9 & 28 & -64 & -43\end{array}$

$\begin{array}{rrrrrr}3 & -8 & 9 & 28 & -64 & -43 \\ 4 & -8 & 9 & 60 & 95 & -29\end{array}$

$\begin{array}{llllll}5 & -8 & 9 & 42 & 1.3 & -35\end{array}$

$\begin{array}{llllll}6 & -8 & 9 & 65 & -31 & -30 \\ 7 & -8 & 9 & 65 & -11 & -22\end{array}$

$\begin{array}{rrrrrr}7 & -8 & 9 & 65 & -11 & -22 \\ -10 & -7 & 9 & 58 & 6 & -29\end{array}$

$\begin{array}{llllll}-9 & -7 & 9 & 53 & -25 & -29\end{array}$

$\begin{array}{llllll}-8 & -7 & 9 & 42 & 4 & -36\end{array}$

$\begin{array}{llllll}-7 & -7 & 9 & 12 & -1 & -46 \\ -6 & -7 & 9 & 27 & 66 & -41\end{array}$

$\begin{array}{rrrrrr}-6 & -7 & 9 & 27 & 66 & -41 \\ -5 & -7 & 9 & 135 & -134 & 11\end{array}$

$\begin{array}{llllll}-4 & -7 & 9 & 243 & 236 & 7 \\ -3 & -7 & 9 & 204 & -208 & 7\end{array}$

$\begin{array}{llllll}-2 & -7 & 9 & 202 & 196 & 8\end{array}$

$\begin{array}{llllll}-1 & -7 & 9 & 86 & -68 & 14\end{array}$

$\begin{array}{rrrrrr}0 & -7 & 9 & 48 & 32 & -27 \\ 1 & -7 & 9 & 136 & -141 & 13\end{array}$

$\begin{array}{rrrrrr}1 & -7 & 9 & 208 & 218 & 8\end{array}$

$\begin{array}{lllllll}3-7 & 9 & 244 & -250 & 8\end{array}$

$\begin{array}{rrrrrr}4 & -7 & 9 & 205 & 184 & 8 \\ 5 & -7 & 9 & 165 & -173 & 11\end{array}$ $\mathrm{h} \mathrm{k} \perp$ IOFO $10 \mathrm{FC}$ IOS

$\begin{array}{llllll}6 & -7 & 9 & 154 & 194 & 12\end{array}$

$\begin{array}{rrrrrr}7 & -7 & 9 & 65 & -96 & -25 \\ 8 & -7 & 9 & 48 & 54 & -28\end{array}$

$\begin{array}{llllll}-10 & -6 & 9 & 32 & -14 & -40\end{array}$

$\begin{array}{llllll}-9 & -6 & 9 & 65 & 2 & -27\end{array}$

$\begin{array}{llllll}-7 & -6 & 9 & 58 & -9 & -26 \\ -6 & -6 & 9 & 74 & 42 & -16\end{array}$

$\begin{array}{llllll}-5 & -6 & 9 & 58 & -70 & -21\end{array}$

$\begin{array}{llllll}-4 & -6 & 9 & 65 & 93 & -18\end{array}$

$\begin{array}{llllll}-3 & -6 & 9 & 23 & -45 & -38\end{array}$

$\begin{array}{rrrrrr}-2 & -6 & 9 & 89 & 90 & 12 \\ -1 & -6 & 9 & 53 & -42 & -24\end{array}$

$\begin{array}{llllll}0 & -6 & 9 & 95 & 103 & 11\end{array}$

$\begin{array}{llllll}1 & -6 & 9 & 30 & -6 & -33\end{array}$

$\begin{array}{llllll}2 & -6 & 9 & 117 & -3.36 & 11\end{array}$

$\begin{array}{llllll}3 & -6 & 9 & 95 & 100 & 14\end{array}$

$\begin{array}{rrrrrr}4 & -6 & 9 & 108 & 64 & 12 \\ 5 & -6 & 9 & 146 & -335 & 11\end{array}$

$\begin{array}{llllll}6 & -6 & 9 & 80 & 62 & -20\end{array}$

$\begin{array}{llllll}7 & -6 & 9 & 89 & -37 & 3.8\end{array}$

$\begin{array}{llllll}8 & -6 & 9 & 59 & 13 & -29\end{array}$

$\begin{array}{rrrrrr}9 & -6 & 9 & 56 & -10 & -33 \\ -10 & -5 & 9 & 62 & -9 & -28\end{array}$

$\begin{array}{llllll}-9 & -5 & 9 & 61 & -40 & -29\end{array}$

$\begin{array}{llllll}-8 & -5 & 9 & 12 & 48 & -46\end{array}$

$\begin{array}{llllll}-7 & -5 & 9 & 12 & -29 & -47\end{array}$

$\begin{array}{rrrrrr}-6 & -5 & 9 & 74 & 43 & -16 \\ -5 & -5 & 9 & 106 & -121 & 12\end{array}$

$\begin{array}{lllllll}-4 & -5 & 9 & 252 & 252 & 6\end{array}$

$\begin{array}{lllll}-3 & -5 & 9 & 243 & -242\end{array}$

$\begin{array}{lllll}-2 & -5 & 9 & 256 & 257\end{array}$

$\begin{array}{llllll}-1 & -5 & 9 & 274 & -267\end{array}$

$\begin{array}{rrrrr}0 & -5 & 9 & 405 & 412 \\ 1 & -5 & 9 & 275 & -283\end{array}$

$\begin{array}{lllll}2 & -5 & 9 & 224 & 198\end{array}$

$\begin{array}{llllll}3 & -5 & 9 & 162 & -146 & 8\end{array}$

$\begin{array}{llllll}4 & -5 & 9 & 28 & 25 & -36\end{array}$

$\begin{array}{rrrrrr}5 & -5 & 9 & 39 & -44 & -34 \\ 6 & -5 & 9 & 248 & 254 & 8\end{array}$

$\begin{array}{llllll}7 & -5 & 9 & 181 & -192 & 10\end{array}$

$\begin{array}{llllll}8 & -5 & 9 & 123 & 223 & 15\end{array}$

$\begin{array}{rrrrrr}10 & -5 & 9 & 27 & 42 & -47\end{array}$

$\begin{array}{rrrrrr}-10 & -4 & 9 & 47 & 19 & -32 \\ -9 & -4 & 9 & 58 & -24 & -27\end{array}$

$\begin{array}{llllll}-8 & -4 & 9 & 53 & -13 & -28\end{array}$

$\begin{array}{llllll}-7 & -4 & 9 & 91 & 53 & 15\end{array}$

$\begin{array}{rrrrrr}-6 & -4 & 9 & 45 & 49 & -28 \\ -5 & -4 & 9 & 121 & -125 & 10\end{array}$

$\begin{array}{rrrrrr}-5 & -4 & 9 & 121 & -125 & 10 \\ -4 & -4 & 9 & 46 & 52 & -26 \\ -3 & -4 & 9 & 70 & -12 & 13\end{array}$

$\begin{array}{rrrrrr}-3 & -4 & 9 & 70 & -12 & 13 \\ -2 & -4 & 9 & 77 & 18 & -18\end{array}$

$\begin{array}{llllll}-1 & -4 & 9 & 11 & -53 & -42\end{array}$

$\begin{array}{llllll}10 & -4 & 9 & 98 & 121 & 10\end{array}$

$\begin{array}{llllll}1 & -4 & 9 & 352 & -155 & 7 \\ 2 & -4 & 9 & 178 & 164 & 7\end{array}$

$\begin{array}{llllll}3 & -4 & 9 & 87 & -115 & 13\end{array}$

$\begin{array}{llllll}4 & -4 & 9 & 94 & -24 & 21\end{array}$

$\begin{array}{rrrrrr}5 & -4 & 9 & 63 & -1.8 & -16 \\ 6 & -4 & 9 & 114 & -127 & 13\end{array}$

$\begin{array}{rrrrrr}7 & -4 & 9 & 153 & 255 & 10 \\ 8 & -4 & 9 & 85 & -26 & 16\end{array}$

$\begin{array}{llllll}9 & -4 & 9 & 35 & -36 & -40\end{array}$

$\begin{array}{rrrrrr}-10 & -3 & 9 & 14 & 76 & -55\end{array}$

$\begin{array}{rrrrrr}-9 & -3 & 9 & 112 & -68 & 14 \\ -8 & -3 & 9 & 74 & 57 & -19\end{array}$

$\begin{array}{rrrrrr}-8 & -3 & 9 & 74 & 57 & -19 \\ -7 & -3 & 9 & 30 & -42 & -39\end{array}$

$\begin{array}{llllll}-6 & -3 & 9 & 166 & 191 & 10\end{array}$

$\begin{array}{lllll}-5 & -3 & 9 & 1.45 & -139\end{array}$

$\begin{array}{lllll}-4 & -3 & 9 & 232 & 247\end{array}$

$\begin{array}{rrrrr}-3 & -3 & 9 & 340 & -342 \\ -2 & -3 & 9 & 296 & 283\end{array}$

$\begin{array}{lllll}-1 & -3 & 9 & 314 & -330\end{array}$

$\begin{array}{lllll}0 & -3 & 9 & 40 & 462\end{array}$

1. $-3 \quad 9 \quad 309-319$

$\begin{array}{rrrrr}2 & -3 & 9 & 156 & 162 \\ 3 & -3 & 9 & 323 & -306\end{array}$

$\begin{array}{llllll}4 & -3 & 9 & 405 & 424\end{array}$

$\begin{array}{lllllll}5 & -3 & 9 & 333 & -319 & 6\end{array}$

$\begin{array}{lllllll}6 & -3 & 9 & 288 & 303 & 7\end{array}$

$\begin{array}{rrrrrr}7 & -3 & 9 & 209 & -191 & 8 \\ 8 & -3 & 9 & 95 & 64 & 25\end{array}$

$\begin{array}{rrrrrr}9 & -3 & 9 & 84 & -27 & -17\end{array}$

$\begin{array}{llllll}1.0 & -3 & 9 & 75 & 38 & -18\end{array}$

$\begin{array}{llllll}11 & -3 & 9 & 72 & -48 & -21\end{array}$

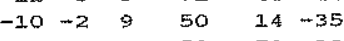

$\begin{array}{rrrrrr}-9 & -2 & 9 & 50 & -70 & -32 \\ -8 & -2 & 9 & 111 & 81 & 12\end{array}$

$\begin{array}{llllll}-7 & -2 & 9 & 58 & -58 & -27\end{array}$

$\begin{array}{llllll}-6 & -2 & 9 & 59 & 35 & -22\end{array}$

$\begin{array}{llllll}-6 & -2 & 9 & 59 & -45 & -22 \\ -5 & -2 & & \end{array}$ $\mathrm{h} \mathrm{k}$ I 1OFO IOFC lOs

$\begin{array}{llllll}-4 & -2 & 9 & 70 & 108 & -15\end{array}$

$\begin{array}{rrrrrr}-3 & -2 & 9 & 84 & 86 & 12 \\ -2 & -2 & 9 & 181 & -382 & 6\end{array}$

$\begin{array}{llllll}-1 & -2 & 9 & 32 & 75 & -31\end{array}$

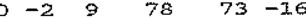

$\begin{array}{rrrrrr}1 & -2 & 9 & 35 & 5 & -25 \\ 2 & -2 & 9 & 125 & -146 & 7\end{array}$

$\begin{array}{lllll}3 & -2 & 9 & 98 & 97\end{array}$

$\begin{array}{llllll}4 & -2 & 9 & 184 & -183 & 7\end{array}$

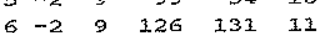

$\begin{array}{rrrrrr}6 & -2 & 9 & 126 & 131 & 11 \\ 7 & -2 & 9 & 36 & 15 & -27\end{array}$

$\begin{array}{lllllll}8 & -2 & 9 & 148 & -137 & 12\end{array}$

$\begin{array}{llllll}9 & -2 & 9 & 92 & 46 & 16\end{array}$

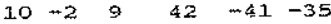

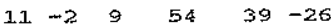

$\begin{array}{rrrrrr}12 & -2 & 9 & 25 & 5 & -38 \\ -10 & -1 & 9 & 62 & 3 & -22\end{array}$

$\begin{array}{llllll}-9 & -1 & 9 & 29 & -59 & -41\end{array}$

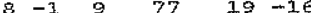

$\begin{array}{llllll}-7 & -1 & 9 & 113 & -132 & 13\end{array}$

$\begin{array}{rrrrr}-6 & -1 & 9 & 293 & 295 \\ -5 & -1 & 9 & 447 & -451\end{array}$

$\begin{array}{lllll}-4 & -1 & 9 & 306 & 328\end{array}$

$\begin{array}{lllll}-3 & -1 & 9 & 301 & -306\end{array}$

$\begin{array}{lllll}-2 & -1 & 9 & 456 & 469\end{array}$

$\begin{array}{rrrrr}-1 & -1 & 9 & 427 & -462 \\ 0 & -1 & 9 & 706 & 764\end{array}$

$\begin{array}{rrrrr}0 & -1 & 9 & 706 & 764 \\ 1 & -1 & 9 & 534 & -566\end{array}$

$\begin{array}{lllll}2 & -1 & 9 & 225 & 225\end{array}$

$\begin{array}{lllll}3 & -1 & 9 & 242 & -232\end{array}$

$4-1 \quad 9 \quad 288 \quad 288$

$\begin{array}{rrrrr}5 & -1 & 9 & 220 & -233 \\ 6 & -1 & 9 & 258 & 247\end{array}$

$\begin{array}{lllll}7 & -1 & 9 & 152 & -154\end{array}$

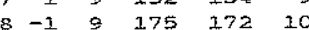

$\begin{array}{lllllll}9 & -1 & 9 & 186 & -192 & 11\end{array}$

$\begin{array}{rrrrrr}10 & -1 & 9 & 79 & 57 & -25 \\ 11 & -1 & 9 & 59 & -27 & -23\end{array}$

$12-1 \quad 9 \quad 74 \quad 31-26$

$\begin{array}{llllll}-30 & 0 & 9 & 69 & -23 & -18\end{array}$

$\begin{array}{llllll}-9 & 0 & 9 & 55 & \text { 1.1. } & -26\end{array}$

$\begin{array}{rrrrrr}-8 & 0 & 9 & 71 & -55 & -18 \\ -7 & 0 & 9 & 37 & 28 & -24\end{array}$

$\begin{array}{llllll}-6 & 0 & 9 & 48 & 12 & -18\end{array}$

$\begin{array}{llllll}-5 & 0 & 9 & 68 & -66 & 11\end{array}$

$\begin{array}{llllll}-4 & 0 & 9 & 53 & 52 & -17\end{array}$

$\begin{array}{rrrrrr}-3 & 0 & 9 & 142 & -114 & 5 \\ -2 & 0 & 9 & 59 & -127 & 11\end{array}$

$\begin{array}{rrrrrr}-1 & 0 & 9 & 302 & 313 & 3\end{array}$

$\begin{array}{llllll}1 & 0 & 9 & 204 & 202 & 4\end{array}$

$\begin{array}{llllll}2 & 0 & 9 & 46 & -61 & -14\end{array}$

$\begin{array}{rrrrrr}3 & 0 & 9 & 168 & -150 & 4 \\ 4 & 0 & 9 & 140 & 61 & 14\end{array}$

$\begin{array}{llllll}5 & 0 & 9 & 127 & 112 & 15\end{array}$

$\begin{array}{rrrrrr}6 & 0 & 9 & 87 & -14 & 9 \\ 7 & 0 & 9 & 66 & -7 & 13\end{array}$

$\begin{array}{rrrrrr}7 & 0 & 9 & 66 & -7 & 13 \\ 8 & 0 & 9 & 126 & -108 & 9\end{array}$

$\begin{array}{rrrrrr}9 & 0 & 9 & 107 & 92 & 10 \\ 10 & 0 & 9 & 87 & -39 & 12\end{array}$

$\begin{array}{llllll}11 & 0 & 9 & 19 & 3 & -33\end{array}$

$\begin{array}{llllll}12 & \circ & 9 & 42 & 9 & -28\end{array}$

$\begin{array}{rrrrrr}-10 & 1 & 9 & 58 & 36 & -30 \\ -9 & 1 & 9 & 52 & -58 & -32\end{array}$

$\begin{array}{llllll}-8 & 1 & 9 & 40 & 42 & -36\end{array}$

$\begin{array}{llllll}-6 & 1 & 9 & 237 & 222 & 8\end{array}$

$\begin{array}{lllll}-5 & 1 & 9 & 291 & -231\end{array}$

$\begin{array}{rrrrr}-4 & 1 & 9 & 353 & 368 \\ -3 & 1 & 9 & 385 & -376\end{array}$

$\begin{array}{lllll}-2 & 1 & 9 & 272 & 286\end{array}$

$\begin{array}{llllll}-1 & 1 & 9 & 534 & -558\end{array}$

019614657

$\begin{array}{rrrrrr}1 & 1 & 9 & 360 & -428 & 5 \\ 2 & 1 & 9 & 210 & 233 & 5\end{array}$

$319252-2715$

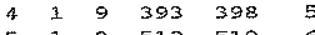

$\begin{array}{rrrrrr}5 & 1 & 9 & 512 & -510 & 6 \\ 6 & 1 & 9 & 571 & 558 & 7\end{array}$

7 1 $9265-258 \quad 6$

$\begin{array}{llllll}8 & 1 & 9 & 87 & 62 & 13\end{array}$

$919263-180 \quad 11$

$\begin{array}{llllll}10 & 1 & 9 & 121 & 142 & 13\end{array}$

$\begin{array}{llllll}11 & 1 & 9 & 34 & -73 & -43\end{array}$

$\begin{array}{rrrrrr}12 & 1 & 9 & 65 & 37 & -23 \\ 13 & 1 & 9 & 50 & -23 & -33\end{array}$

$\begin{array}{llllll}-9 & 2 & 9 & 66 & 59 & -28\end{array}$

$\begin{array}{llllll}-8 & 2 & 9 & 50 & -91 & -33\end{array}$

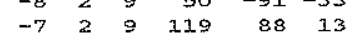




\begin{tabular}{|c|c|c|c|c|c|c|c|c|c|}
\hline-6 & 2 & 9 & 75 & 80 & -17 & -5 & 6 & 9 & 21 \\
\hline-5 & 2 & 9 & 163 & 145 & 9 & -4 & 6 & 9 & 54 \\
\hline-4 & 2 & 9 & 32 & 16 & -31 & -3 & 6 & 9 & 49 \\
\hline-3 & 2 & 9 & 91 & 69 & 11 & -2 & 6 & 9 & 33 \\
\hline-2 & 2 & 9 & 69 & 72 & 12 & -1 & 6 & 9 & 117 \\
\hline-1 & 2 & 9 & 58 & -40 & -19 & 0 & 6 & 9 & 151 \\
\hline 0 & 2 & 9 & 28 & 69 & -27 & 1 & 6 & 9 & 149 \\
\hline 1 & 2 & 9 & 206 & 221 & 6 & 2 & 6 & 9 & 104 \\
\hline 2 & 2 & 9 & 452 & -446 & 5 & 3 & 6 & 9 & 138 \\
\hline 3 & 2 & 9 & 214 & 207 & 5 & 4 & 6 & 9 & 140 \\
\hline 4 & 2 & 9 & 279 & -261 & 5 & 5 & 6 & 9 & 161 \\
\hline 5 & 2 & 9 & 39 & 35 & -17 & 6 & 6 & 9 & 192 \\
\hline 6 & 2 & 9 & 136 & -130 & 9 & 7 & 6 & 9 & 130 \\
\hline 7 & 2 & 9 & 101 & -28 & 12 & 8 & 6 & 9 & 34 \\
\hline 8 & 2 & 9 & 48 & 49 & -21 & 9 & 6 & 9 & 138 \\
\hline 9 & 2 & 9 & 109 & -65 & 1.4 & 10 & 6 & 9 & 73 \\
\hline 10 & 2 & 9 & 46 & -22 & -28 & 11 & 6 & 9 & 41 \\
\hline 11 & 2 & 9 & 39 & -9 & -29 & 12 & 6 & 9 & 55 \\
\hline 12 & 2 & 9 & 53 & 35 & -30 & 13 & 6 & 9 & 58 \\
\hline-9 & 3 & 9 & 13 & -61 & -53 & -7 & 7 & 9 & 75 \\
\hline-8 & 3 & 9 & 42 & -5 & -36 & -6 & 7 & 9 & 136 \\
\hline-7 & 3 & 9 & 23 & 0 & -43 & 6 & 7 & 9 & 202 \\
\hline-6 & 3 & 9 & 26 & 105 & -38 & 7 & 7 & 9 & 260 \\
\hline-5 & 3 & 9 & 220 & -209 & 7 & 8 & 7 & 9 & 124 \\
\hline-4 & 3 & 9 & 366 & 398 & 6 & 9 & 7 & 9 & 225 \\
\hline-3 & 3 & 9 & 234 & -219 & 6 & Io & 7 & 9 & 128 \\
\hline-2 & 3 & 9 & 282 & 287 & 5 & 11 & 7 & 9 & 73 \\
\hline-2 & 3 & 9 & 497 & -505 & 6 & 12 & 7 & 9 & 50 \\
\hline 0 & 3 & 9 & 732 & 752 & 5 & 13 & 7 & 9 & 41 \\
\hline 1 & 3 & 9 & 580 & -613 & 5 & -6 & 8 & 9 & 93 \\
\hline 2 & 3 & 9 & 358 & 392 & 5 & -5 & 8 & 9 & 73 \\
\hline 3 & 3 & 9 & 287 & -221 & 6 & $-\frac{\pi}{x}$ & 8 & 9 & 44 \\
\hline 4 & 3 & 9 & 255 & 295 & 5 & -3 & 8 & 9 & 59 \\
\hline 5 & 3 & 9 & 247 & -253 & 5 & -2 & 8 & 9 & 162 \\
\hline 6 & 3 & 9 & 304 & 292 & 5 & -1 & 8 & 9 & 91 \\
\hline 7 & 3 & 9 & 276 & -298 & 6 & 0 & 8 & 9 & 91. \\
\hline 8 & 3 & 9 & 79 & 106 & 15 & 1 & 8 & 9 & 1.1 \\
\hline 9 & 3 & 9 & 88 & -59 & 15 & 2 & 8 & 9 & 31 \\
\hline 10 & 3 & 9 & 35 & 109 & -38 & 3 & 8 & 9 & 89 \\
\hline 11 & 3 & 9 & 52 & -76 & -31 & 4 & 8 & 9 & 114 \\
\hline 12 & 3 & 9 & 38 & 43 & -32 & 5 & 8 & 9 & 11 \\
\hline 13 & 3 & 9 & 55 & -30 & -33 & 6 & 8 & 9 & 87 \\
\hline-9 & 4 & 9 & 54 & -1 & -26 & 7 & 8 & 9 & 92 \\
\hline-8 & 4 & 9 & 36 & -37 & -32 & 8 & 8 & 9 & 94 \\
\hline-7 & 4 & 9 & 95 & 64 & 15 & 9 & 8 & 9 & 103 \\
\hline-6 & $\neq$ & 9 & 22 & -32 & -44 & 10 & 8 & 9 & 56 \\
\hline-5 & 4 & 9 & 49 & 一王1 & -28 & 11 & 8 & 9 & 59 \\
\hline-4 & 4 & 9 & 313 & -304 & 11 & 12 & 8 & 9 & 83 \\
\hline-3 & 4 & 9 & 51 & 34 & -25 & 13 & 8 & 9 & 14 \\
\hline-2 & 4 & 9 & 63 & 28 & -13 & -5 & 9 & 9 & 86 \\
\hline-1 & 4 & 9 & 39 & -19 & -25 & -4 & 9 & 9 & 85 \\
\hline 0 & 4 & 9 & 72 & 6 & -15 & -3 & 9 & 9 & 161 \\
\hline 1 & 4 & 9 & 197 & -166 & 5 & -2 & 9 & 9 & 1.49 \\
\hline 2 & 4 & 9 & 68 & 60 & 11 & -1 & 9 & 9 & 174 \\
\hline 3 & 4 & 9 & 163 & -176 & 5 & 0 & 9 & 9 & 172 \\
\hline 4 & 4 & 9 & 208 & -36 & 8 & 1 & 9 & 9 & 197 \\
\hline 5 & 4 & 9 & 106 & 118 & 9 & $z$ & 9 & 9 & 58 \\
\hline 6 & 4 & 9 & 53 & -19 & -23 & 3 & 9 & 9 & 317 \\
\hline 7 & 4 & 9 & 115 & -14 & -10 & 4 & 9 & 9 & 371 \\
\hline 8 & 4 & 9 & 129 & -119 & 11 & 5 & 9 & 9 & 236 \\
\hline 9 & 4 & 9 & 32 & -22 & -36 & 6 & 9 & 9 & 190 \\
\hline 10 & $\stackrel{4}{4}$ & 9 & 78 & 49 & -28 & 7 & 9 & 9 & 125 \\
\hline 11 & 4 & 9 & 12 & 34 & -49 & 8 & 9 & 9 & 40 \\
\hline 12 & 4 & 9 & 49 & 7 & -33 & 9 & 9 & 9 & 13 \\
\hline 13 & 4 & 9 & 52 & -8 & -34 & 10 & 9 & 9 & 29 \\
\hline-8 & 5 & 9 & 86 & 98 & -19 & 11 & 9 & 9 & 126 \\
\hline-7 & 5 & 9 & 90 & -73 & 17 & 12 & 9 & 9 & 42 \\
\hline-6 & 5 & 9 & 199 & 191 & 9 & 13 & 9 & 9 & 66 \\
\hline-5 & 5 & 9 & 148 & -159 & 1.1 & -4 & 10 & 9 & 31 \\
\hline-4 & 5 & 9 & 147 & 143 & 10 & -3 & 10 & 9 & 74 \\
\hline-3 & 5 & $\vartheta$ & 336 & -355 & 6 & -2 & 10 & 9 & 34 \\
\hline-2 & 5 & 9 & 319 & 312 & 5 & -1 & 10 & 9 & 42 \\
\hline-1 & 5 & 9 & 523 & -500 & 6 & 0 & 10 & 9 & 98 \\
\hline 0 & 5 & 9 & 491 & 489 & 6 & 1 & 10 & 9 & 53 \\
\hline 1 & 5 & 9 & 418 & -413 & 6 & 2 & 10 & 9 & 83 \\
\hline 2 & 5 & 9 & 236 & 252 & 5 & 3 & 10 & 9 & 48 \\
\hline 3 & 5 & 9 & 383 & -388 & 5 & 4 & 10 & 9 & 141 \\
\hline 4 & 5 & 9 & 467 & 519 & 6 & 5 & 10 & 9 & 60 \\
\hline 5 & 5 & 9 & 317 & -342 & 5 & 6 & 10 & 9 & 123 \\
\hline 6 & 5 & 9 & 269 & 277 & 6 & 7 & 10 & 9 & 53 \\
\hline 7 & 5 & 9 & 128 & -83 & 9 & 8 & 30 & 9 & 96 \\
\hline 8 & 5 & 9 & 138 & 243 & 10 & 9 & 10 & 9 & 82 \\
\hline 9 & 5 & 9 & 244 & -243 & 8 & 10 & 10 & 9 & 56 \\
\hline 10 & 5 & 9 & 95 & 68 & 15 & I王 & 10 & 9 & 47 \\
\hline 11 & 5 & 9 & 28 & -55 & -42 & -3 & 11 & 9 & 120 \\
\hline 12 & 5 & 9 & 40 & 44 & -32 & -2 & \pm 1 & 9 & 200 \\
\hline 13 & 5 & 9 & 92 & 15 & 18 & -1 & 11 & 9 & 122 \\
\hline-8 & 6 & 9 & 61. & -28 & -23 & 0 & 11 & 9 & 159 \\
\hline-7 & 6 & 9 & 110 & 61 & 15 & 1 & 11 & 9 & 139 \\
\hline
\end{tabular}




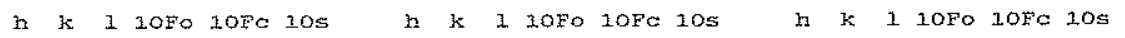

\begin{tabular}{|c|c|c|c|c|c|c|c|c|c|}
\hline 1 & 11 & & & -310 & 5 & 2 & 51 & 10 & 251 \\
\hline 2 & 13 & 20 & 102 & -123 & 8 & 3 & 51 & 10 & 187 \\
\hline 3 & $I 1$ & 20 & 124 & 93 & 7 & 4 & 51 & 20 & 140 \\
\hline 4 & 11 & 10 & 173 & -177 & 6 & 5 & 51 & 10 & 70 \\
\hline 5 & 11 & 10 & 101 & 55 & 11 & 6 & 51 & 10 & 43 \\
\hline 6 & 11 & 10 & 136 & -46 & -9 & 7 & 51 & 10 & 98 \\
\hline 7 & 11 & 10 & 258 & -155 & 9 & 8 & 51 & 10 & 145. \\
\hline 8 & 11 & 10 & 76 & -23 & 15 & 9 & 53 & 10 & 42 \\
\hline 9 & 1 & 10 & 69 & 28 & -20 & 10 & 51 & 10 & 49 \\
\hline 10 & 11 & 10 & 53 & -3 & -31 & 21 & 51 & 10 & 77 \\
\hline II & 3 & 10 & 80 & 32. & -18 & 12 & 51 & 10 & 49 \\
\hline 12 & 1 & 10 & 57 & 10 & -29 & 13 & 51 & 10 & 57 \\
\hline-9 & 2 & 10 & 48 & -45 & $-3 \frac{1}{x}$ & -7 & 63 & 10 & 104 \\
\hline-8 & 22 & 20 & 77 & 41 & -19 & -6 & 61 & 10 & 73 \\
\hline-7 & 2 & 10 & 53 & -24 & -28 & -5 & & 10 & 177 \\
\hline-6 & 2 & 10 & 53 & 33 & -22 & -4 & 61 & 10 & 202 \\
\hline-5 & 23 & 10 & 179 & -167 & 9 & -3 & 61 & 1.0 & 227 \\
\hline-4 & 2 & 10 & 231 & 221 & 7 & -2 & 61 & 10 & 188 \\
\hline-3 & 2 & 10 & 184 & -190 & 7 & -1 & 63 & 10 & 87 \\
\hline-2 & 23 & 10 & 323 & 328 & 5 & 0 & 63 & 10 & 335 \\
\hline-1 & 23 & 10 & 238 & -264 & 5 & 1 & 63 & 10 & 315 \\
\hline 0 & 2 & 10 & 314 & 363 & 5 & 2 & 63 & 10 & 522 \\
\hline 2 & 27 & Io & $\$ 00$ & -435 & 6 & 3 & 63 & 20 & 244 \\
\hline 2 & 23 & 10 & 41.7 & 453 & 6 & 4 & 63 & 10 & 256 \\
\hline 3 & 23 & 10 & 548 & -570 & 6 & 5 & & 10 & 278 \\
\hline 4 & 21 & 10 & 350 & 369 & 5 & 6 & 61 & 10 & 260 \\
\hline 5 & 23 & 10 & 244 & -245 & 6 & 7 & 61 & 10 & 170 \\
\hline 6 & 23 & 20 & 334 & 338 & 5 & 8 & & 10 & 128 \\
\hline 7 & 23 & 10 & 282 & -311 & 6 & 9 & 63 & 10 & 132 \\
\hline 8 & 23 & 20 & 225 & 99 & 10 & 10 & & 10 & 76 \\
\hline 9 & 23 & 10 & 148 & -163 & 21 & 11 & 61 & 10 & 13 \\
\hline 20 & 23 & 10 & 128 & 125 & 13 & 12 & 61 & 10 & 53 \\
\hline 12 & 27 & 10 & 50 & -41 & -31 & 13 & 61 & 10 & 77 \\
\hline 12 & 23 & 10 & 47 & 38 & -35 & -7 & & 10 & 62 \\
\hline 13 & 23 & 10 & 73 & -29 & -29 & -6 & 73 & 10 & 66 \\
\hline-9 & 31 & 10 & 102 & 17 & 15 & -5 & 73 & 10 & 41 \\
\hline-8 & 37 & 10 & 60 & -20 & -27 & -4 & 73 & 10 & 42 \\
\hline-7 & $3=$ & 20 & 129 & 89 & 11 & -3 & 7 & 10 & 120 \\
\hline-6 & $3:$ & 10 & 63 & -67 & -20 & -2 & 73 & 10 & 106 \\
\hline-5 & $3=$ & 10 & 54 & 72 & -27 & -1 & 73 & 10 & 73 \\
\hline-4 & 3 & 10 & 176 & -172 & 8 & 0 & 7 & 10 & 96 \\
\hline-3 & 31 & 10 & 69 & -33 & 13 & 1 & 73 & 10 & 169 \\
\hline-2 & $3=$ & 30 & 41 & -18 & -18 & 2 & & 10 & 58 \\
\hline-1 & $3=$ & 10 & 127 & 112 & 8 & 3 & 7 & 10 & 64 \\
\hline 0 & $3=$ & 10 & 22 & 4 & -32 & 4 & 73 & 10 & 120 \\
\hline 1. & 3 & 10 & 1.58 & -170 & 7 & 5 & $7:$ & 10 & 74 \\
\hline 2 & 3 & 10 & 72 & 8 & -15 & 6 & 7 & 10 & 45 \\
\hline 3 & 3 & 10 & 85 & 63 & 10 & 7 & 7 & 10 & 120 \\
\hline 4 & 3 & 10 & 201 & -1.12 & -6 & 8 & 7 & 10 & 59 \\
\hline 5 & 3 & 10 & 46 & 24 & -24 & 9 & 7 & 10 & 82 \\
\hline 6 & 3 & 10 & 211 & -82 & 10 & 10 & 7 & 10 & 90 \\
\hline 7 & 3 & 10 & 187 & 199 & 8 & 11 & 7 & 10 & 86 \\
\hline 8 & 3 & 10 & 138 & -123 & 10 & 12 & 7 & 10 & 12 \\
\hline 9 & 3 & 10 & 115 & -94 & 11 & 13 & 7 & 10 & 59 \\
\hline 10 & $3:$ & 10 & 2.5 & 73 & 13 & -6 & 8 & 20 & 84 \\
\hline 12 & 3 & 10 & 55 & -36 & -29 & -5 & 8 & 10 & 106 \\
\hline 12 & 3 & 10 & 100 & 45 & -22 & -4 & 8 & 10 & 133 \\
\hline 13 & 3 & 20 & 33 & -8 & -53 & -3 & 8 & 10 & 168 \\
\hline-8 & 4 & 10 & 111 & 82 & 15 & -2 & 8 & 10 & 215 \\
\hline-7 & 4 & 10 & 53 & -19 & -31 & -1 & 8 & 10 & 250 \\
\hline-6 & 4 & 10 & 71 & 49 & -20 & 0 & 8 & 10 & 343 \\
\hline-5 & 4 & 10 & 46 & -8 & -22 & 1 & 8 & 10 & 294 \\
\hline-4 & 4 & 10 & 51 & 86 & -29 & 2 & 8 & 10 & 70 \\
\hline-3 & 4 & 10 & 217 & -213 & 6 & 3 & 8 & 20 & 344 \\
\hline-2 & 4 & 10 & 168 & 169 & 7 & 4 & 8 & 10 & 296 \\
\hline-1 & 4 & 10 & 257 & -275 & 5 & 5 & 8 & 10 & 295 \\
\hline 0 & 4 & 10 & 337 & 355 & 5 & 6 & 8 & 10 & 304 \\
\hline 1 & 4 & 20 & 507 & -522 & 6 & 7 & 8 & 30 & 103 \\
\hline 2 & 4 & IO & 22 & 68 & -32 & 8 & 8 & 30 & 61 \\
\hline 3 & 4 & 10 & 209 & -221 & 5 & & 8 & 20 & 65 \\
\hline 4 & 4 & 10 & 340 & 348 & 5 & \pm 0 & 8 & 10 & 72 \\
\hline 5 & 4 & 10 & 208 & -223 & 6 & 11 & 8 & 10 & 59 \\
\hline 6 & 4 & 10 & 257 & 265 & 6 & 12 & 8 & 10 & 75 \\
\hline 7 & 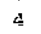 & 10 & 265 & -165 & 7 & 13 & 8 & 10 & 117 \\
\hline 8 & 4 & 10 & 144 & 163 & 10 & -5 & 9 & 10 & 32 \\
\hline 9 & 4 & 10 & 25 & -3 & -33 & -4 & 9 & 10 & 52 \\
\hline 10 & 4 & 10 & 44 & 39 & -27 & -3 & 9 & 10 & 78 \\
\hline 12 & 4 & 10 & 52 & -70 & -32 & -2 & 9 & 10 & 22 \\
\hline 12 & 4 & 30 & 77 & 65 & -19 & -1 & 9 & 10 & 74 \\
\hline 13 & 4 & 10 & 50 & -14 & -32 & 0 & 9 & 10 & 182 \\
\hline-8 & 5 & 10 & 78 & -7 & -27 & 1 & & 10 & 128 \\
\hline-7 & 5 & Io & 82 & -8 & -18 & 2 & 9 & 10 & 56 \\
\hline-5 & 5 & 10 & 49 & 50 & -32 & 3 & 9 & 10 & 119 \\
\hline-5 & 5 & 10 & 55 & -1 & -21 & 4 & 9. & 20 & 159 \\
\hline$-\frac{1}{4}$ & 5 & 30 & 10 & -61 & -42 & 5 & 9 & 10 & 51 \\
\hline-3 & 5 & 10 & 120 & 132 & 10 & 6 & 9 & 10 & 28 \\
\hline-2 & 5 & 20 & 53 & -5 & -16 & 7 & 9 & 10 & 112 \\
\hline-1 & 5 & 10 & 34 & 45 & -22 & 8 & 9 & 10 & 84 \\
\hline 0 & 5 & 10 & 225 & 242 & 6 & 9 & 9 & 10 & 48 \\
\hline
\end{tabular}

h $k$ I 1OFO 1OFC 1OS

$\begin{array}{llllll}0 & -9 & 11 & 124 & 1.52 & 14\end{array}$

$\begin{array}{llllll}1 & -9 & 11 & 157 & -130 & 11\end{array}$

$\begin{array}{llllll}2 & -9 & 11 & 93 & 99 & 18\end{array}$

$\begin{array}{llllll}3 & -9 & 21 & 103 & -102 & 1.7\end{array}$

$\begin{array}{llllll}4 & -9 & 12 & 105 & 81 & 27\end{array}$

$\begin{array}{rrrrrr}5 & -9 & 11 & 11.7 & -107 & 15 \\ -8 & -8 & 11 & 41 & 20 & -39\end{array}$

$\begin{array}{llllll}-7 & -8 & 11 & 13 & -28 & -53\end{array}$

$\begin{array}{llllll}-6 & -8 & 11 & 81 & 69 & -17\end{array}$

$\begin{array}{llllll}-5 & -8 & 11 & 58 & -78 & -27\end{array}$

$\begin{array}{rrrrrr}-4 & -8 & 11 & 21 & 65 & -38 \\ -3 & -8 & 11 & 71 & -99 & -19\end{array}$

$\begin{array}{llllll}-2 & -8 & 23 & 96 & 103 & 14\end{array}$

$\begin{array}{llllll}-1 & -8 & 11 & 96 & -122 & 15\end{array}$

$\begin{array}{llllll}0 & -8 & 11 & 72 & 76 & -24\end{array}$

$\begin{array}{rrrrrr}1 & -8 & 11 & 66 & -6 & -24\end{array}$

$\begin{array}{rrrrrr}2 & -8 & 11 & 51 & 2 & -31 \\ 3 & -8 & 11 & 37 & -8 & -34\end{array}$

$\begin{array}{llllll}4 & -8 & 11 & 57 & -20 & -28\end{array}$

$\begin{array}{llllll}5 & -8 & 31 & 50 & 22 & -34\end{array}$

$\begin{array}{rrrrrr}6 & -8 & 11 & 98 & -24 & 15 \\ -8 & -7 & 11 & 12 & 19 & -47\end{array}$

$\begin{array}{rllllll}-8 & -7 & 11 & 12 & 19 & -47 \\ -7 & -7 & 11 & 13 & 4 & -52\end{array}$

$\begin{array}{llllll}-6 & -7 & 1.3 & 59 & 68 & -21\end{array}$

$\begin{array}{lllllll}-5 & -7 & 11 & 144 & -139 & 11\end{array}$

$\begin{array}{lllll}-4 & -7 & 11 & 194 & 194\end{array}$

$\begin{array}{lllll}-3 & -7 & 11 & 227 & -253\end{array}$

$\begin{array}{lllll}-2 & -7 & 11 & 205 & 180\end{array}$

$\begin{array}{lllll}-1 & -7 & 11 & 209 & -195\end{array}$

$\begin{array}{lllll}0 & -7 & 11 & 212 & 231\end{array}$

$\begin{array}{lllll}1 & -7 & 11 & 224 & -233\end{array}$

$\begin{array}{lllll}2 & -7 & 11 & 178 & 181\end{array}$

$\begin{array}{llllll}3 & -7 & 11 & 130 & -1.52 & 13\end{array}$

$\begin{array}{llllll}5 & -7 & 11 & 132 & -147 & 15\end{array}$

$\begin{array}{cccccc}6 & -7 & 21 & 96 & 312 & 28\end{array}$

$\begin{array}{llllll}7 & -7 & 12 & 112 & -83 & 15\end{array}$

$\begin{array}{llllll}-9 & -6 & 21 & 26 & -29 & -48\end{array}$

$\begin{array}{llllll}-8 & -6 & 11 & 42 & -10 & -36 \\ -7 & -6 & 11 & 37 & 19 & -38\end{array}$

$\begin{array}{llllll}-6 & -6 & 11 & 82 & -9 & -22\end{array}$

$\begin{array}{llllll}-5 & -6 & 11 & 12 & 51 & -49\end{array}$

$\begin{array}{llllll}-4 & -6 & 11 & 46 & -39 & -31\end{array}$

$\begin{array}{llllll}-2 & -6 & 11 & 51 & 26 & -25\end{array}$

$\begin{array}{llllll}-1 & -6 & 11 & 211 & -228 & 8\end{array}$

$\begin{array}{rrrrr}0 & -6 & 11 & 180 & 197 \\ 1 & -6 & 11 & 89 & -84\end{array}$

$\begin{array}{rrrrrr}2 & -6 & 11 & 29 & 13 & -38\end{array}$

$\begin{array}{llllll}3 & -6 & 11 & 26 & -28 & -38\end{array}$

$\begin{array}{llllll}4 & -6 & 11 & 45 & -26 & -25\end{array}$

$\begin{array}{rrrrrr}5 & -6 & 213 & 58 & 64 & -30 \\ 6 & -6 & 31 & 47 & -26 & -32\end{array}$

$\begin{array}{llllll}7 & -6 & 12 & 63 & 15 & -30\end{array}$

$\begin{array}{llllll}8 & -6 & 11 & 32 & -1.4 & -44\end{array}$

$\begin{array}{llllll}-9 & -5 & 11 & 72 & -37 & -27\end{array}$

$\begin{array}{lllllll}-8 & -5 & 11 & 57 & 64 & -30\end{array}$

$\begin{array}{llllll}-7 & -5 & 11 & 70 & -76 & -20\end{array}$

$\begin{array}{rrrrrr}-6 & -5 & 11 & 98 & 82 & 13 \\ -5 & -5 & 11 & 139 & -92 & 31\end{array}$

$\begin{array}{llllll}-4 & -5 & 12 & 117 & 95 & 12\end{array}$

$\begin{array}{llllll}-3 & -5 & 11 & 84 & -73 & 13\end{array}$

$\begin{array}{lllll}-2 & -5 & 11 & 160 & 168\end{array}$

$\begin{array}{lllll}-1 & -5 & 11 & 281 & -285\end{array}$

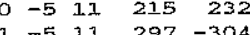

$\begin{array}{lllll}2 & -5 & 11 & 318 & 305\end{array}$

$\begin{array}{lllll}3 & -5 & 11 & 205 & -208\end{array}$

$\begin{array}{llllll}4 & -5 & 11 & 139 & 136 & 11 \\ 5 & -5 & 11 & 229 & -229 & 8\end{array}$

$\begin{array}{rrrrrr}6 & -5 & 11 & 175 & 172 & 11\end{array}$

$\begin{array}{llllll}7 & -5 & 11 & 97 & -139 & 18\end{array}$

$\begin{array}{llllll}8 & -5 & 11 & 43 & 103 & -37\end{array}$

$\begin{array}{llllll}9 & -5 & 11 & 32 & -82 & -48 \\ -51 & 33 & -51 & -40\end{array}$

$\begin{array}{rrrrrr}-8 & -4 & 11 & 33 & -51 & -40 \\ -7 & -4 & 3.3 & 57 & 48 & -23\end{array}$

$\begin{array}{lllllllll}-6 & -4 & 11 & 37 & 2 & -28\end{array}$

$\begin{array}{llllll}-5 & -4 & 11 & 25 & -32 & -37\end{array}$

$\begin{array}{rrrrrr}-4 & -4 & 1.1 & 81 & -72 & 14 \\ -3 & -4 & 11 . & 70 & 79 & -15\end{array}$

$\begin{array}{llllll}-2 & -4 & 11 & 34 & -37 & -30\end{array}$

$\begin{array}{llllll}-1 & -4 & 11 & 43 & 31 & -27\end{array}$

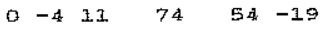

$\begin{array}{rrrrrr}1 & -4 & 11 & 81 & -73 & 12 \\ 2 & -4 & 11 & 23 & -17 & -38\end{array}$

$\begin{array}{llllll}3 & -4 & 11 & 48 & 19 & -26 \\ 4 & -4 & 11 & 72 & 71 & -16\end{array}$

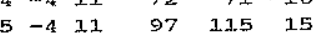

$\begin{array}{rrrrrr}6 & -4 & 11 & 137 & -164 & 12 \\ 7 & -4 & 11 & 122 & 125 & 34\end{array}$
K 1 10FO 10FC tos

$\begin{array}{llllll}8 & -4 & 11 & 101 & -53 & 14\end{array}$ $\begin{array}{llllll}9 & -4 & 11 & 57 & -11 & -23\end{array}$

$\begin{array}{rrrrrr}9 & -4 & 11 & 68 & 20 & -24 \\ -9 & -3 & 31 & 53 & -69 & -27\end{array}$

$\begin{array}{llllll}-8 & -3 & 11 & 55 & 39 & -29\end{array}$

$\begin{array}{llllll}-7 & -3 & 11 & 42 & -50 & -33\end{array}$

$\begin{array}{rrrrrr}-6 & -3 & 11 & 47 & 58 & -29 \\ -5 & -3 & 11 & 90 & -109 & 14\end{array}$

$\begin{array}{llllll}-4 & -3 & 11 & 114 & 85 & 11\end{array}$

$\begin{array}{lllllll}-3 & -3 & 11 & 171 & -178 & 7\end{array}$

$\begin{array}{rrrrrr}-2 & -3 & 11 & 155 & 153 & 7 \\ -2 & -3 & 11 & 337 & -357 & 5\end{array}$

$\begin{array}{rrrrr}-2 & -3 & 11 & 337 & -357 \\ 0 & -3 & 11 & 463 & 482\end{array}$

$\begin{array}{llllll}1 & -3 & 11 & 324 & -350 & 5\end{array}$

$\begin{array}{llllll}2 & -3 & 11 & 341 & 345 & 5\end{array}$

$\begin{array}{lllll}3 & -3 & 11 & 465 & -480\end{array}$

$\begin{array}{rrrrrr}4 & -3 & 11 & 289 & 280 & 6 \\ 5 & -3 & 11 & 255 & -257 & 7\end{array}$

$\begin{array}{lllllll}6 & -3 & 11 & 259 & 268 & 7\end{array}$

$\begin{array}{llllll}7 & -3 & 11 & 214 & -100 & 13\end{array}$

$\begin{array}{lllllll}9 & -3 & 13 & 66 & -16 & -24\end{array}$

$\begin{array}{rrrrrr}10 & -3 & 11 & 54 & 15 & -33 \\ 11 & -3 & 11 & 100 & -70 & 17\end{array}$

$\begin{array}{lllllllll}-9 & -2 & 11 & 12 & -43 & -49\end{array}$

$\begin{array}{llllll}-8 & -2 & 11 & 38 & 41 & -31\end{array}$

$\begin{array}{llllll}-7 & -2 & 11 & 63 & -33 & -27\end{array}$

$\begin{array}{rrrrrr}-6 & -2 & 11 & 105 & 62 & 13 \\ -5 & -2 & 11 & 42 & 9 & -30\end{array}$

$\begin{array}{lllllll}-4 & -2 & 31 & 134 & -138 & 9\end{array}$

$\begin{array}{llllll}-3 & -2 & 11 & 101 & 128 & 12\end{array}$

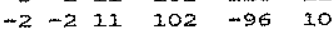

$\begin{array}{llllll}-1 & -2 & 11 & 59 & -92 & -14\end{array}$

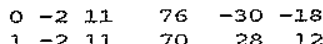

$\begin{array}{llllll}2 & -2 & 11 & 99 & 86 & 9\end{array}$

$\begin{array}{llllll}3 & -2 & 13 & 45 & -2 & -19\end{array}$

$\begin{array}{lllllll}4 & -2 & 11 & 25 & -12 & -37\end{array}$

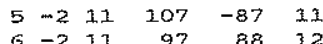

$\begin{array}{llllll}7 & -2 & 11 & 51 & -11 & -28\end{array}$

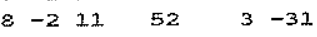

$\begin{array}{llllll}9 & -2 & 11 & 40 & 36 & -36\end{array}$

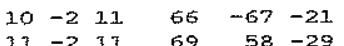

$\begin{array}{llllll}-9 & -1 & 11 & 35 & -9 & -38\end{array}$

$\begin{array}{llllll}-8 & -1 & 21 & 58 & 12 & -26\end{array}$

$\begin{array}{llllll}-7 & -1 & 11 & 98 & -112 & 16\end{array}$

$\begin{array}{lllll}-6 & -1 & 11 & 269 & 255\end{array}$

$\begin{array}{lllll}-5 & -1 & 11 & 200 & -225\end{array}$

$\begin{array}{lllll}-4 & -1 & 11 & 224 & 231\end{array}$

$\begin{array}{lllll}-3 & -1 & 11 & 188 & -182\end{array}$

$\begin{array}{lllll}-2 & -1 & 11 & 253 & 250\end{array}$

$\begin{array}{lllll}-1 & -1 & 11 & 269 & -296\end{array}$

$\begin{array}{llllr}0 & -1 & 11 & 300 & 344 \\ 1 & -1 & 33 & 383 & -425\end{array}$

$\begin{array}{lllll}2 & -1 & 11 & 228 & 248\end{array}$

$\begin{array}{lllll}3-1 & 11 & 436 & -444\end{array}$

$\begin{array}{rrrrr}4 & -1 & 11 & 436 & 445 \\ 5 & -1 & 11 & 344 & -350\end{array}$

$\begin{array}{llllll}6 & -1 & 11 & 237 & 229 & 6\end{array}$

$\begin{array}{llllll}7 & -1 & 11 & 1.68 & -152 & 9\end{array}$

$\begin{array}{llllll}8 & -1 & 11 & 164 & 151 & 10\end{array}$

$\begin{array}{llllll}9 & -1 & 11 & 167 & -162 & 11\end{array}$

$\begin{array}{rrrrrr}10 & -1 & 11 & 115 & 1.14 & 13 \\ 11 & -1 & 11 & 36 & -27 & -39\end{array}$

$\begin{array}{lllllll}11 & -1 & 11 & 27 & 37 & -49\end{array}$

$\begin{array}{llllll}-9 & 0 & 11 & 33 & 1 & -29\end{array}$

$\begin{array}{llllll}-8 & 0 & 11 & 41 & -17 & -24\end{array}$

$\begin{array}{llllll}-7 & 0 & 11 & 52 & 61 & -18\end{array}$

$\begin{array}{llllll}-6 & 0 & 11 & 97 & -50 & 14 \\ -5 & 0 & 11 & 70 & -43 & -18\end{array}$

$\begin{array}{llllll}-4 & 0 & 1.1 & 59 & 23 & -16\end{array}$

$\begin{array}{llllll}-3 & 0 & 11 & 45 & -28 & -23\end{array}$

$\begin{array}{rrrrrr}-2 & 0 & 11 & 38 & 48 & -23 \\ -1 & 0 & 11 & 73 & -36 & 14\end{array}$

$\begin{array}{rrrrrr}-1 & 0 & 11 & 73 & -36 & 14 \\ 0 & 0 & 11 & 212 & 165 & 8\end{array}$

$\begin{array}{llllll}1 & 0 & 11 & 27 & -94 & -23\end{array}$

$\begin{array}{llllll}2 & 0 & 11 & 76 & 2 & -26\end{array}$

$\begin{array}{rrrrrr}3 & 0 & 11 & 121 & -129 & 12 \\ 4 & 0 & 11 & 63 & -48 & -17\end{array}$

$\begin{array}{rrrrrr}5 & 0 & 11 & 148 & 116 & 7 \\ 6 & 0 & 11 & 97 & -57 & 8\end{array}$

$\begin{array}{llllll}7 & 0 & 11 & 52 & -22 & -18\end{array}$

$\begin{array}{rrrrrr}8 & 0 & 12 & 117 & -84 & 9 \\ 9 & 0 & 32 & 11 & 20 & -32\end{array}$

$\begin{array}{rrrrrr}10 & 0 & 11 & 41 & 1 & -25 \\ 11 & 0 & 11 & 54 & -25 & -20\end{array}$

$120 \begin{array}{lllll}11 & 54 & 39 & -24\end{array}$

$\begin{array}{rrrrrr}-9 & 1 & 11 & 104 & -77 & 16 \\ -8 & 1 & 11 & 91 & 67 & 17\end{array}$ 
$\mathrm{h} \mathrm{k} 1 \mathrm{lOFO}$ lOF' $10 \mathrm{~S}$

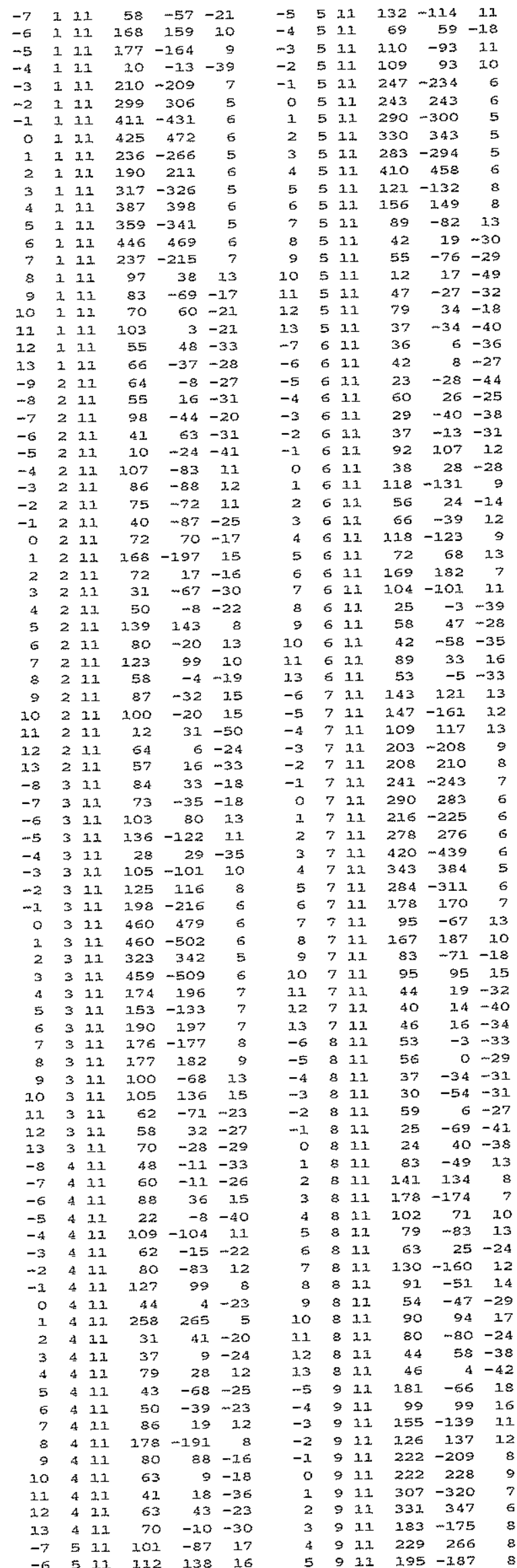

h $\mathrm{k} I$ I $10 \mathrm{FO} 10 \mathrm{Fc} 10 \mathrm{~s}$

$\begin{array}{llllll}-4 & -8 & 12 & 99 & 119 & 15\end{array}$

$\begin{array}{lllll}6 & 9 & 11 & 283 & 297\end{array}$ $\begin{array}{llll}6 & 11 & 283 & 297 \\ 7 & 11 & 108 & -74\end{array}$ $8 \quad 9 \quad 31 \quad 83 \quad 66 \quad 36$ $\begin{array}{llllll}9 & 9 & 11 & 49 & -36 & -26\end{array}$ $\begin{array}{llllll}10 & 9 & 11 & 93 & -18 & 14\end{array}$ $\begin{array}{llllll}11 & 9 & 11 & 39 & -11 & -36\end{array}$ $\begin{array}{llllll}12 & 9 & 11 & 51 & 39 & -35 \\ 3 & 9 & 11 & 75 & -43 & -23\end{array}$ $\begin{array}{llllll}13 & 9 & 11 & 75 & -43 & -23 \\ -4 & 0 & 11 & 90 & -31 & -24\end{array}$ $\begin{array}{rrrrrr}-4 & 10 & 11 & 90 & -31 & -24 \\ -3 & 10 & 11 & 63 & 26 & -28\end{array}$ $\begin{array}{lllllll}-3 & 10 & 11 & 63 & 26 & -28 \\ -2 & 10 & 11 & 79 & -5 & -22\end{array}$

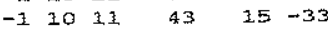
$\begin{array}{llllll}0 & 10 & 11 & 72 & -17 & -1.7\end{array}$ $\begin{array}{llllll}1 & 10 & 11 & 21 & -38 & -42\end{array}$ $\begin{array}{llllll}2 & 10 & 11 & 38 & 2 & -32\end{array}$

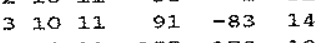

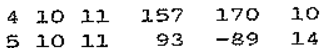
$\begin{array}{llllll}6 & 10 & 11 & 36 & 78 & -38\end{array}$ $\begin{array}{llllll}710 & 11 & 145 & -146 & 11\end{array}$ $\begin{array}{llllll}8 & 10 & 21 & 119 & 151 & 24\end{array}$ $\begin{array}{rrrrrr}9 & 10 & 11 & 57 & -11 & -30 \\ 10 & 10 & 11 & 48 & 7 & -32\end{array}$ $\begin{array}{llllll}11 & 10 & 11 & 74 & 14 & -28\end{array}$ $\begin{array}{llllll}12 & 10 & 11 & 56 & 6 & -33\end{array}$ $\begin{array}{rrrrrr}-3 & 11 & 11 & 49 & -68 & -35 \\ -2 & 11 & 11 & 47 & 97 & -35\end{array}$ $\begin{array}{llllll}-2 & 11 & 11 & 47 & 97 & -35 \\ -1 & 11 & 11 & 32 & -91 & -34\end{array}$

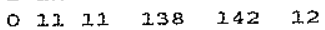
$\begin{array}{llllll}1 & 11 & 11 & 161 & -168 & 10\end{array}$ $\begin{array}{rrrrrr}2 & 11 & 11 & 225 & 223 & 9 \\ 3 & 11 & 11 & 231 & -245 & 8\end{array}$

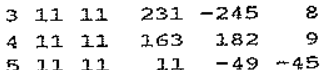
$\begin{array}{rrrrrr}5 & 11 & 11 & 11 & -49 & -4.5 \\ 6 & 11 & 11 & 99 & 103 & 14\end{array}$ $\begin{array}{llllll}6 & 11 & 11 & 99 & 103 & 14 \\ 7 & 11 & 11 & 75 & -36 & -18\end{array}$ $\begin{array}{llllll}8 & 31 & 11 & 98 & -15 & 14 \\ 9 & 13 & 11 & 51 & -72 & -35\end{array}$ $\begin{array}{rrrrrr}9 & 11 & 11 & 51 & -72 & -35 \\ 10 & 11 & 11 & 110 & 107 & 15\end{array}$ $\begin{array}{llllll}11 & 11 & 11 & 81 & -18 & -19\end{array}$

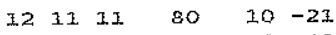
$\begin{array}{rrrrrr}-1 & 12 & 11 & 10 & 45 & -42 \\ 0 & 12 & 11 & 74 & -11 & -20\end{array}$ $\begin{array}{llllll}1 & 12 & 11 & 61 & -12 & -26\end{array}$ $\begin{array}{lllllllll}2 & 3.2 & 11 & 58 & 6 & -27\end{array}$

$\begin{array}{lllll}3 & 1211 & 50 & 20 & -28\end{array}$ $\begin{array}{llllll}4 & 12 & 11 & 56 & 47 & -26\end{array}$ $\begin{array}{llllll}5 & 12 & 11 & 115 & -127 & 14 \\ 6 & 12 & 11 & 138 & 124 & 12\end{array}$

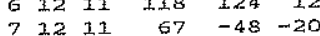

$\begin{array}{llllll}8 & 12 & 11 & 34 & 28 & -41\end{array}$

$\begin{array}{llllll}9 & 12 & 11 & 68 & -30 & -22\end{array}$

$\begin{array}{llllll}10 & 12 & 1.2 & 63 & 7 & -28\end{array}$

$\begin{array}{llllll}11 & 12 & 11 & 38 & -10 & -42\end{array}$

$\begin{array}{llllll}2 & 13 & 11 & 49 & 78 & -35\end{array}$

$\begin{array}{llllll}3 & 13 & 11 & 129 & -131 & 13\end{array}$

$\begin{array}{rrrrrr}4 & 13 & 11 & 123 & 132 & 12 \\ 5 & 13 & 11 & 44 & -69 & -35\end{array}$

$\begin{array}{llllll}6 & 13 & 11 & 65 & 80 & -22\end{array}$

$\begin{array}{llllll}7 & 13 & 11 & 57 & -109 & -31\end{array}$

$\begin{array}{llllll}8 & 13 & 11 . & 58 & 28 & -30\end{array}$ $\begin{array}{rrrrrr}9 & 13 & 11 & 31 & 14 & -44 \\ 0 & 13 & 11 & 65 & -25 & -29\end{array}$ $\begin{array}{llllll}3 & 14 & 1.1 & 182 & -13 & -1.7\end{array}$ $\begin{array}{llllll}4 & 14 & 11 & 24 & 14 & -45 \\ 5 & 14 & 11 & 49 & -13 & -33\end{array}$ $\begin{array}{llllrl}5 & 14 & 11 & 49 & -13 & -33 \\ 6 & 14 & 11 & 67 & 43 & -20\end{array}$ \begin{tabular}{llllll}
7 & 14 & 11 & 47 & -13 & -33 \\
\hline & 14 & 12 & 51 & 31 & -36
\end{tabular}

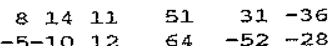
$\begin{array}{lllll}-4-10 & 12 & 42 & 71 & -35\end{array}$ $\begin{array}{lllll}-3-10 & 12 & 66 & -81 & -28\end{array}$ $\begin{array}{rrrrr}-3-10 & 12 & 66 & -81 & -28 \\ 0-10 & 12 & 43 & 87 & -38\end{array}$

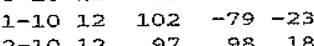

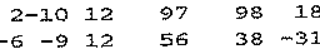
$\begin{array}{llllll}-5 & -9 & 12 & 92 & -23 & -23\end{array}$ $\begin{array}{llllll}-4 & -9 & 12 & 34 & 5.2 & -33\end{array}$ $\begin{array}{llllll}-3 & -9 & 12 & 44 & -66 & -35\end{array}$ $\begin{array}{rrrrrr}-2 & -9 & 12 & 13 & 38 & -45 \\ -1 & -9 & 12 & 67 & -30 & -25\end{array}$ $\begin{array}{llllll}0 & -9 & 12 & 12 & 30 & -46\end{array}$ $\begin{array}{rrrrrr}0 & -9 & 12 & 12 & 30 & -46 \\ 1 & -9 & 12 & 38 & -46 & -38\end{array}$ $\begin{array}{llllll}2 & -9 & 12 & 30 & 33 & -44\end{array}$ $\begin{array}{rrrrrr}3 & -9 & 12 & 62 & -3 & -28 \\ 4 & -9 & 12 & 37 & -17 & -42\end{array}$ $\begin{array}{rrrrrrr}-7 & -8 & 12 & 108 & -55 & 14\end{array}$ $\begin{array}{rrrrrr}-6 & -8 & 12 & 83 & 90 & -19 \\ -5 & -8 & 12 & 75 & -95 & -19\end{array}$

\begin{tabular}{|c|c|c|c|c|c|c|c|c|c|c|}
\hline $4-$ & $\begin{array}{ll}-8 & 12\end{array}$ & & 99 & 119 & 15 & $-2-3$ & 12 & $140-$ & -127 & 8 \\
\hline & -812 & & $99-$ & -137 & 15 & $-1-3$ & 12 & 161 & 379 & 8 \\
\hline-2 & -812 & & 319 & 220 & 13 & $0-3$ & 12 & 123 & -117 & 9 \\
\hline- & $-8 \quad 1$ & & $156-$ & -142 & 21 & $1-3$ & 12 & 45 & 14 & -25 \\
\hline $0-$ & $\begin{array}{ll}-8 & 1\end{array}$ & 2 & 165 & 169 & 20 & $z-3$ & 12 & 108 & -112 & 12 \\
\hline 1 & -81 & 2 & $166-$ & -169 & 11 & $3-3$ & 12 & 126 & 220 & 9 \\
\hline $2-$ & -83 & 2 & 103 & 120 & 15 & $4-3$ & 12 & 68 & $-8.3-$ & -16 \\
\hline $3-$ & -81 & 2 & 106 & -126 & 15 & $5-3$ & 12 & 25 & $-18-$ & -40 \\
\hline 4 & $\begin{array}{ll}-81 \\
\text {. }\end{array}$ & 2 & 150 & 132 & 12 & $6-3$ & 312 & 92 & 104 & 16 \\
\hline & -81 & 2 & $140-$ & -116 & 13 & $7-3$ & 312 & 61 & $61-$ & -21 \\
\hline $\begin{array}{r}6 \\
-87\end{array}$ & -81 & 2 & 95 & 101 & 19 & $8-3$ & 12 & 75 & -80 & -18 \\
\hline $\begin{array}{l}-8 \\
-7\end{array}$ & -71 & 2 & 63 & 7 & -24 & $9-3$ & 12 & 13 & 66 & -52 \\
\hline $\begin{array}{l}-7 \\
-6\end{array}$ & -72 & 2 & 23 & -5 & -39 & $10-3$ & 12 & 41 & -26 & -39 \\
\hline $\left.\begin{array}{l}-6 \\
-5\end{array}\right]$ & -71 & 12 & 53 & 13 & -29 & $-9-2$ & $2 \pm 2$ & 44 & -24 & -35 \\
\hline $\left.\begin{array}{l}-5 \\
-4\end{array}\right]$ & $\begin{array}{ll}-7 & 1\end{array}$ & 12 & 32 & -35 & -38 & $-8-2$ & 212 & 1.23 & 78 & 13 \\
\hline $\begin{array}{l}-4 \\
-3\end{array}$ & -71 & 2 & 95 & 97 & 15 & $-7-2$ & 212 & 117 & -98 & 14 \\
\hline $\begin{array}{l}-3 \\
-2\end{array}$ & $-7 I$ & 2 & 37 & -73 & -37 & $-6-2$ & 232 & 93 & 93 & 1.5 \\
\hline $\begin{array}{l}-2 \\
-1\end{array}$ & -71 & 12 & $\$ 0$ & 20 & -28 & $-5-2$ & 212 & 98 & -96 & 13 \\
\hline $\begin{array}{c}-1 \\
0\end{array}$ & $\begin{array}{ll}-7 & 1 \\
-7 & 1\end{array}$ & 12 & 53. & -28 & -27 & $-4-2$ & 212 & 138 & 153 & 9 \\
\hline 1. & $\begin{array}{ll}-7 & 1 \\
-7 & 3\end{array}$ & 12 & 85 & 46 & -21 & $-3-2$ & 212 & 159 & -160 & 8 \\
\hline 2. & -71 & 2 & $76-$ & -109 & -18 & $-2-2$ & 212 & 99 & 87 & 10 \\
\hline 3 & -71 & 12 & $\begin{array}{l}91 \\
36\end{array}$ & 77 & 25 & $-3-2$ & $\begin{array}{ll}212 \\
2.12\end{array}$ & $\begin{array}{r}50 \\
773\end{array}$ & -66 & -23 \\
\hline 4 & -71 & 12 & $\begin{array}{l}36 \\
33\end{array}$ & $\begin{array}{l}-33 \\
-38\end{array}$ & $\begin{array}{l}-35 \\
-39\end{array}$ & $\begin{array}{ll}0 & -1 \\
1 & -\end{array}$ & $\begin{array}{ll}212 \\
2 & 12\end{array}$ & $\begin{array}{l}171 \\
225\end{array}$ & $\begin{array}{r}170 \\
-229\end{array}$ & $\begin{array}{l}7 \\
6\end{array}$ \\
\hline 5 & -73 & 2 & 58 & 54 & $\begin{array}{l}-39 \\
-25\end{array}$ & $\begin{array}{ll}1 & -2 \\
2 & -2\end{array}$ & 212 & 246 & 254 & 6 \\
\hline 6 & -71 & .2 & 33 & -21 & -53 & $3-2$ & 212 & 349 & -354 & 5 \\
\hline 7 & -71 & 12 & 32 & $1 z$ & -39 & $4-$ & 212 & 294 & 290 & 6 \\
\hline-8 & -61 & 12 & 109 & 94 & 16 & $5-2$ & 212 & 321 & -342 & 6 \\
\hline $\begin{array}{l}-7 \\
-6\end{array}$ & -63 & 2 & 114 & -69 & 14 & $6-3$ & 212 & 167 & 148 & 10 \\
\hline $\begin{array}{l}-6 \\
-5\end{array}$ & $-6 I$ & 2 & 36 & 45 & -36 & $7-3$ & 212 & 141 & -99 & 10 \\
\hline $\begin{array}{l}-5 \\
-4\end{array}$ & -61 & 12 & 38 & -78 & -36 & $8-7$ & 212 & 99 & 2.22 & 34 \\
\hline $\begin{array}{l}-4 \\
-3\end{array}$ & $\begin{array}{ll}-6 & 1\end{array}$ & 2 & 132 & 122 & 11 & $9-2$ & 212 & 84 & -70 & 16 \\
\hline $\begin{array}{l}-3 \\
-2 \\
-2\end{array}$ & -61 & 12 & 168 & -169 & 9 & $10-$ & 212 & 1.3 & 5 & -50 \\
\hline $\begin{array}{l}-2 \\
-1\end{array}$ & -63 & 22 & 213 & 220 & 8 & $31-$ & 212 & 13 & -7 & -53 \\
\hline $\begin{array}{r}-1 . \\
0\end{array}$ & -61 & 12 & 194 & -193 & 8 & -9 & 112 & 57 & -9 & -32 \\
\hline $\begin{array}{l}0 \\
1\end{array}$ & $\begin{array}{ll}-6 & 1\end{array}$ & 12 & 240 & 259 & 8 & $-8-$ & 112 & 30 & 19 & -44 \\
\hline $\begin{array}{l}3 \\
2\end{array}$ & $-6 x$ & 22 & 333 & -346 & 7 & $-7-1$ & 112 & 67 & 31. & -18 \\
\hline & -63 & 32 & 259 & 265 & 8 & $-6-1$ & 112 & 37 & 72 & -36 \\
\hline $\begin{array}{l}3 \\
4\end{array}$ & $\begin{array}{ll}-6 & 1 \\
-6 & 1\end{array}$ & 32 & 199 & -200 & 9 & $-5-$ & 112 & 124 & -143 & 12 \\
\hline $\begin{array}{l}4 \\
5\end{array}$ & $\begin{array}{ll}-6 & 1 \\
-6 & 3\end{array}$ & 12 & 176 & 181 & 10 & $-4-$ & 112 & 90 & 225 & 25 \\
\hline 6 & $\begin{array}{ll}-0 \\
-6 & 3\end{array}$ & 12 & $\begin{array}{l}166 \\
3.64\end{array}$ & -154 & 11 & $\begin{array}{ll}-3 & - \\
-2 & -\end{array}$ & $\begin{array}{l}1212 \\
112\end{array}$ & 41 & $\begin{array}{r}-70 \\
-703\end{array}$ & -28 \\
\hline & -63 & 12 & $\begin{array}{l}3.64 \\
105\end{array}$ & $\begin{array}{l}163 \\
-99\end{array}$ & 12 & $\begin{array}{ll}-2 & - \\
-1 & -\end{array}$ & $\begin{array}{ll}1 & 12 \\
1 & 1.2\end{array}$ & $\begin{array}{l}97 \\
34\end{array}$ & $\begin{array}{r}-103 \\
54\end{array}$ & $\begin{array}{r}10 \\
-29\end{array}$ \\
\hline 8 & -61 & 12 & 49 & 50 & $\begin{array}{r}16 \\
-35\end{array}$ & $0-1$ & 112 & 174 & $\begin{array}{l}34 \\
28\end{array}$ & -12 \\
\hline-9 & -5 & 12 & 41 & -1.1 & -38 & $1-3$ & 112 & 26 & 39 & -32 \\
\hline-8 & $-5=$ & 12 & 25 & 22 & -45 & $2-$ & $1 \quad 12$ & 126 & $m 141$ & 9 \\
\hline-7 & -5 & 12 & 55 & -24 & -25 & $3-$ & 112 & 246 & 373 & 8 \\
\hline-6 & -5 & 12 & 59 & 31 & -27 & $4-$ & 112 & 36 & 32 & -30 \\
\hline-5 & -5 & 12 & 48 & -16 & -29 & $5-$ & $1 \quad 12$ & 106 & 35 & 11 \\
\hline-4 & $-5=$ & 12 & 57 & -34 & -26 & 6 & $1 \quad 12$ & 119 & 108 & 11 \\
\hline-3 & -5 & 1.2 & 47 & 31 & -28 & $7-$ & 112 & 71 & -49 & -17 \\
\hline-2 & -5 & 12 & 95 & -86 & 12 & $8-$ & $1 \quad 12$ & 80 & -30 & -37 \\
\hline-1 & -5 & 12 & 11 & 55 & -46 & $9-$ & 112 & 58 & 5 & -27 \\
\hline 0 & -5 & 32 & 31 & 75 & -37 & 10 - & 112 & 63 & 5 & -22 \\
\hline 1 & -5 & 12 & 40 & -84 & -25 & $\pm 1-$ & $-1 \quad 12$ & 88 & 22 & -18 \\
\hline 2 & -5 & 12 & 11 & 5 & -43 & 12 & $-1 \quad 12$ & 78 & -33 & -22 \\
\hline 3 & -5 & 12 & 56 & -55 & -27 & -9 & 012 & 74 & -38 & -19 \\
\hline 4 & -5 & 12 & 11. & 73 & -45 & -8 & 012 & 52 & 9 & -19 \\
\hline 5 & -5 & 12 & 66 & -69 & -21 & -7 & 01.2 & 50 & -10 & -18 \\
\hline 6 & -5 & 12 & 56 & 30 & -29 & -6 & 012 & 107 & 1.06 & 2.1 \\
\hline 7 & -5 & 12 & 91 & 48 & 25 & -5 & 012 & 192 & -1.76 & 1.2 \\
\hline 8 & -5 & 12 & 65 & -29 & -22 & -4 & 012 & 220 & 248 & 8 \\
\hline & -5 & 12 & 77 & 21 & -27 & -3 & 012 & 356 & -353 & 4 \\
\hline-9 & -4 & 12 & 54 & -47 & -33 & -2 & 012 & 204 & 204 & 5 \\
\hline-8 & -4 & 12 & 30 & 54 & -4.4 & -1 & 012 & 238 & -261 & 4 \\
\hline-7 & -4 & 12 & 59 & -53 & -28 & o & 012 & 32.4 & $\quad 333$ & 4 \\
\hline-6 & -4 & 12 & 61 & 91 & -29 & 1 & 012 & 373 & -419 & 4 \\
\hline & -4 & 12 & 129 & -1.24 & 11 & 2 & 012 & 184 & 206 & 5 \\
\hline-4 & -4 & 12 & 130 & 122 & 11 & 3 & 012 & 236 & -248 & 6 \\
\hline-3 & -4 & 22 & 80 & -79 & 14 & 4 & 012 & 124 & 1.15 & 16 \\
\hline & -4 & 12 & 61 & -37 & -22 & 5 & 012 & 199 & -210 & 5 \\
\hline-1 & -4 & 12 & 29 & -30 & -33 & 6 & 01.2 & 424 & 449 & 5 \\
\hline 0 & -4 & 12 & 189 & 176 & 7 & 7 & 012 & 182 & -178 & 7 \\
\hline 1 & -4 & 12 & 314 & -316 & 6 & 8 & 012 & 112 & 101 & 20 \\
\hline 2 & -4 & 12 & 342 & 346 & 6 & 9 & 012 & 132 & $2-132$ & 9 \\
\hline 3 & -4 & 12 & 260 & -271 & 7 & 10 & 012 & 78 & 68 & 14 \\
\hline 4 & -4 & 12 & 184 & 185 & 9 & 11 & 012 & 53 & -60 & -21 \\
\hline 5 & -4 & 12 & 149 & -153 & 20 & 12 & 012 & 89 & & -26 \\
\hline 6 & -4 & 12 & 171 & 159 & 30 & -9 & 132 & 52 & -47 & -32 \\
\hline 7 & -4 & 12 & 153 & -150 & 11 & -8 & 112 & 13 & 10 & -52 \\
\hline 8 & -4 & 12 & 93 & 108 & 18 & -7 & 312 & 33 & -5 & -35 \\
\hline 9 & -4 & 12 & 78 & -52 & -20 & -6 & 112 & 58 & -1 & -25 \\
\hline 10 & -4 & 12 & 65 & 59 & -33 & -5 & 112 & 29 & -32 & -37 \\
\hline-9 & -3 & 12 & 54 & -29 & -33 & -4 & 122 & 42 & $2 \quad 29$ & -28 \\
\hline-8 & -3 & 12 & 59 & -23 & -28 & -3 & 112 & 126 & $5-130$ & \\
\hline-7 & -3 & 12 & 74 & 40 & -19 & -2 & 112 & 11 & 10 & -45 \\
\hline-6 & -3 & 12 & 42 & 23 & -31 & -1 & 112 & 51 & $1-107$ & -19 \\
\hline & -3 & 12 & 39 & -36 & -32 & 0 & 112 & 132 & $2 \quad 228$ & -12 \\
\hline-4 & -3 & 12 & 110 & 92 & 11 & 1 & 112 & 48 & $8-122$ & -29 \\
\hline
\end{tabular}

h k l lOFO lOFC IOS

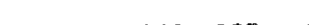
$\begin{array}{llll}-3 & -3 & 140-327\end{array}$ $\begin{array}{lllll}0 & -3 & 12 & 123 & -117\end{array}$ $\begin{array}{llllll}2 & -3 & 12 & 108 & -312 & 11\end{array}$ $\begin{array}{llllll}3 & -3 & 12 & 126 & 120 & 9\end{array}$ $\begin{array}{llllll}4 & -3 & 12 & 68 & -83 & -16\end{array}$ $\begin{array}{llllll}6 & -3 & 12 & 92 & 104 & 16\end{array}$

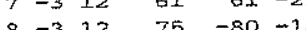
$\begin{array}{llll}112 & 13 & 66 & -52\end{array}$ $\begin{array}{llllll}-9 & -2 & 12 & 44 & -24 & -35\end{array}$ $\begin{array}{llllll}-8 & -2 & 12 & 113 & -24 & 13\end{array}$ $\begin{array}{llllll}-6 & -2 & 32 & 91 & 93 & 1.5\end{array}$ $\begin{array}{llllll}-4 & -2 & 12 & 138 & 153\end{array}$ $\begin{array}{llllll}-3 & -2 & 12 & 159 & -160 \\ -2 & -2 & 12 & 99 & 87 & 10\end{array}$ $\begin{array}{llllll}-1 & -2 & 12 & 50 & -66 & -23\end{array}$ $\begin{array}{lllll}1 & -2 & 12 & 225 & -229\end{array}$ $\begin{array}{llllll}3 & -2 & 12 & 349 & -354 & 5\end{array}$ $\begin{array}{llllll}4 & -2 & 12 & 294 & 290 & 6 \\ 5 & -2 & 12 & 321 & -342 & 6\end{array}$

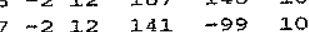

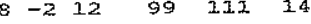

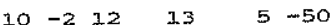

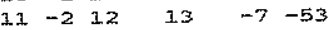

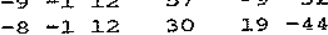

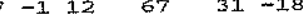
$\begin{array}{llllll}-5 & -1 & 12 & 124 & -143 & 12\end{array}$ $\begin{array}{llllll}-3 & -1 & 12 & 41 & -70 & -28\end{array}$

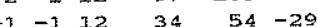
$\begin{array}{rrrrrr}0 & -1 & 12 & 174 & 28 & -11 \\ 1 & -1 & 12 & 26 & 39 & -32\end{array}$ $\begin{array}{llllll}2 & -1 & 12 & 126 & -141 & 9\end{array}$ $\begin{array}{llllll}4 & -1 & 12 & 36 & 32 & -30\end{array}$ $\begin{array}{rrrrrr}5 & -1 & 12 & 106 & 35 & 11 \\ 6 & -1 & 12 & 119 & 108 & 11\end{array}$ $\begin{array}{llllll}7 & -1 & 12 & 71 & -49 & -17\end{array}$ $\begin{array}{llllll}9 & -1 & 12 & 58 & 5 & -27\end{array}$ $\begin{array}{rrrrrr}10 & -1 & 12 & 63 & 5 & -22 \\ 11 & -2 & 12 & 88 & 22 & -18\end{array}$ $\begin{array}{llllll}-9 & 0 & 12 & 74 & -38 & -19 \\ -8 & 0 & 12 & 52 & 9 & -19\end{array}$ $\begin{array}{rrrrrr}-7 & 0 & 12 & 50 & -10 & -18 \\ -6 & 0 & 12 & 107 & 1.06 & 11\end{array}$ $\begin{array}{llllll}-5 & 0 & 12 & 192 & -1.76 & 12\end{array}$ $\begin{array}{lllll}-4 & 0 & 12 & 220 & 248 \\ -3 & 0 & 12 & 356 & -353\end{array}$ $\begin{array}{llllll}-2 & 0 & 12 & 204 & 204\end{array}$ $\begin{array}{llllll}1 & 1 & 12 & 52 & 17 & -21\end{array}$ $\begin{array}{llllll}12 & -1 & 12 & 78 & -33 & -22\end{array}$ 
$\mathrm{b} \mathrm{k}$ I LOFO $10 \mathrm{FC}$ lOS

$\begin{array}{llllll}3 & 1 & 12 & 68 & -55 & 13\end{array}$ $\begin{array}{llllll}4 & 1 & 12 & 75 & 29 & 11 \\ 5 & 1 & 12 & 66 & -17 & -14\end{array}$ $6 \begin{array}{lllll}6 & 12 & 87 & -50 & 13\end{array}$ $7 \quad 1 \quad 12 \quad 155 \quad 16910$ $\begin{array}{rrrrrr}8 & 1 & 12 & 79 & 23 & 15 \\ 9 & 1 & 12 & 60 & 3 & -28\end{array}$ $\begin{array}{rrrrrr}10 & 1 & 12 & 28 & -18 & -41\end{array}$ $\begin{array}{llllll}11 & 1 & 12 & 52 & 32 & -25 \\ 12 & 1 & 12 & 41 & -8 & -38\end{array}$ $\begin{array}{llllll}-8 & 212 & 59 & 68 & -33\end{array}$ $\begin{array}{rrrrrr}-7 & 2 & 12 & 96 & -85 & 17 \\ -6 & 2 & 12 & 123 & 109 & 12\end{array}$

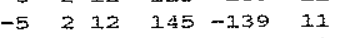
$\begin{array}{rrrrr}-4 & 2 & 12 & 158 & 138 \\ -3 & 2 & 12 & 225 & -204\end{array}$

$\begin{array}{llllll}-2 & 2 & 12 & 66 & 55 & -22\end{array}$

$\begin{array}{llllll}-1 & 2 & 12 & 211 & -221 & 6\end{array}$

$\begin{array}{rrrrrr}0 & 2 & 32 & 407 & 428 \\ 1 & 2 & 12 & 422 & -428\end{array}$

$\begin{array}{lllll}2 & 2 & 12 & 512 & 538\end{array}$

$4212406 \quad 425$

$5 \quad 212 \quad 272-301$

$\begin{array}{rrrrrr}6 & 2 & 12 & 210 & 209 & 7 \\ 7 & 2 & 12 & 198 & -212 & 8\end{array}$

8212100 so 14

$\begin{array}{rrrrrr}9 & 2 & 12 & 57 & -65 & -29 \\ 10 & 2 & 12 & 132 & 122 & 12\end{array}$

$\begin{array}{rrrrrr}11 & 2 & 12 & 72 & -15 & -18 \\ 12 & 2 & 12 & 104 & 11 & 13\end{array}$

$\begin{array}{rrrrrr}12 & 2 & 12 & 104 & 11 & 13 \\ 13 & 2 & 12 & 49 & -6 & -30\end{array}$

$\begin{array}{llllll}-8 & 3 & 12 & 31 & 0 & -41 \\ -7 & 3 & 12 & 45 & 2 & -33\end{array}$

$\begin{array}{rrrrrr}-7 & 3 & 12 & 45 & 2 & -33 \\ -6 & 3 & 12 & 54 & 62 & -28\end{array}$

$\begin{array}{llllll}-5 & 3 & 12 & 48 & -86 & -29 \\ -4 & 3 & 12 & 74 & 104 & -17\end{array}$

$\begin{array}{llllll}-3 & 3 & 12 & 54 & -49 & -19\end{array}$

$\begin{array}{llllll}-2 & 3 & 12 & 45 & -12 & -25\end{array}$

$\begin{array}{rrrrrr}-1 & 3 & 12 & 122 & -123 & 8 \\ 0 & 3 & 12 & 78 & 19 & -16\end{array}$

$\begin{array}{llllll}0 & 3 & 12 & 78 & 19 & -16 \\ 1 & 3 & 12 & 83 & 44 & -18\end{array}$

$\begin{array}{llllll}2 & 3 & 12 & 47 & -83 & -23\end{array}$

$\begin{array}{lllll}3 & 3 & 12 & 118 & 128 \\ 4 & 3 & 12 & 213 & 217\end{array}$

$\begin{array}{rrrrr}5 & 3 & 12 & 132 & -132 \\ 5 & 3 & 12 & 162 & 170\end{array}$

$\begin{array}{llllll}7 & 3 & 12 & 56 & -17 & -25\end{array}$

$\begin{array}{llllll}8 & 3 & 12 & 129 & -123 & 11\end{array}$

$9 \quad 312 \quad 89-42 \quad 15$

$\begin{array}{rrrrrr}10 & 3 & 12 & 48 & -10 & -31 \\ 13 & 3 & 12 & 81 & 15 & 16\end{array}$

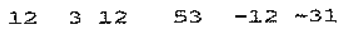

$13 \quad 312 \quad 42 \quad 29-36$

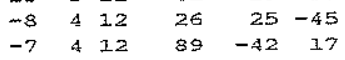

$\begin{array}{llllll}-6 & 4 & 12 & 118 & 111 & 14 \\ -5 & 4 & 12 & 109 & -113 & 14\end{array}$

$\begin{array}{llllll}-4 & 4 & 12 & 156 & 127 & 9\end{array}$

$\begin{array}{rrrrrr}-3 & 4 & 12 & 87 & -91 & 14 \\ -2 & 4 & 12 & 200 & 201 & 7\end{array}$

$\begin{array}{rrrrr}-2 & 4 & 12 & 200 & 201 \\ -1 & 4 & 12 & 137 & -156 \\ 0 & 4 & 12 & 300 & 302\end{array}$

$\begin{array}{rrrrr}0 & 4 & 12 & 300 & 302 \\ 1 & 4 & 12 & 413 & -432\end{array}$

$2412 \quad 343 \quad 347$

$3 * 12427-432$

$\begin{array}{lllll}\frac{1}{7} & 4 & 12 & 437 & 464 \\ 5 & 4 & 12 & 265 & -284\end{array}$

6 $412306 \quad 317$

$7412 \quad 138-116 \quad 9$

$\begin{array}{rrrrrr}3 & 4 & 12 & 43 & 76 & -31 \\ 9 & 4 & 12 & 69 & -30 & -17\end{array}$

$\begin{array}{rrrrrr}10 & 4 & 12 & 63 & 37 & -28 \\ 11 & 4 & 12 & 60 & -35 & -26\end{array}$

$12 \quad 4 \quad 12 \quad 13 \quad 34-51$

$\begin{array}{llllll}13 & 4 & 12 & 71 & -64 & -24\end{array}$

$\begin{array}{lllll}-7 & 5 & 12 & 83 & 3\end{array}-17$

$\begin{array}{rrrrrr}-6 & 5 & 12 & 34 & -6 & -40 \\ -5 & 5 & 12 & 77 & -10 & -16\end{array}$

$\begin{array}{lllll}-4 & 512 & 12 & -32 & -17\end{array}$

$\begin{array}{lllllll}-3 & 5 & 12 & 54 & 43 & -24\end{array}$

$\begin{array}{rrrrrr}-2 & 5 & 12 & 133 & -144 & 10 \\ -1 & 5 & 12 & 256 & 155 & 8\end{array}$

$\begin{array}{rrrrrr}0 & 5 & 12 & 53 & 0 & -21 \\ 1 & 5 & 12 & 24 & 53 & -33\end{array}$

$\begin{array}{lllrrr}2 & 5 & 12 & 124 & -125 & 8\end{array}$

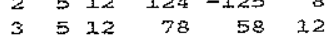

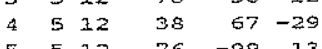

$\begin{array}{rrrrrr}5 & 5 & 12 & 76 & -99 & 13 \\ 6 & 5 & 12 & 119 & 105 & 9\end{array}$

$h$ k 1 2OFO LOFC 205

$\begin{array}{llllll}7 & 5 & 12 & 36 & -42 & -34\end{array}$ $\begin{array}{llllll}8 & 5 & 12 & 74 & 75 & -17\end{array}$ $\begin{array}{llllll}9 & 5 & 12 & 30 & -56 & -39\end{array}$ $\begin{array}{llllll}10 & 5 & 12 & 32 & 45 & -41 \\ 11 & 5 & 12 & 41 & 53 & -37\end{array}$ $\begin{array}{llllll}12 & 5 & 12 & 13 & -29 & -53 \\ 13 & 5 & 12 & 98 & 35 & 18\end{array}$ $\begin{array}{llllll}13 & 5 & 12 & 98 & 35 & 18 \\ -6 & 6 & 12 & 95 & 92 & 16\end{array}$ $\begin{array}{rrrrrr}-5 & 6 & 12 & 177 & -182 & 11\end{array}$ $\begin{array}{rrrrrr}-4 & 6 & 12 & 166 & 174 & 10 \\ -3 & 6 & 12 & 227 & -218 & 8\end{array}$ $\begin{array}{rrrrrr}-3 & 6 & 12 & 227 & -21.8 & 8 \\ -2 & 6 & 12 & 166 & 148 & 9\end{array}$ $\begin{array}{lllllll}-1 & 6 & 32 & 179 & -190 & 8\end{array}$ $\begin{array}{rrrrrr}0 & 6 & 12 & 97 & 89 & 11 \\ 1 & 5 & 12 & 25 & -39 & -25\end{array}$ $\begin{array}{lrrrrr}2 & 6 & 12 & 39 & 41 & -26\end{array}$ $\begin{array}{lllll}6 & 12 & 293 & -272 & 5\end{array}$ $5612 \quad 250-262 \quad 6$ $\begin{array}{rrrrrr}6 & 6 & 12 & 170 & 164 & 8 \\ 7 & 6 & 12 & 156 & -162 & 9\end{array}$

$\begin{array}{llllll}7 & 6 & 12 & 11 & 53 & -45\end{array}$

$\begin{array}{llllll}9 & 6 & 12 & 34 & -29 & -34 \\ 0 & 6 & 12 & 61 & 40 & -22\end{array}$

$\begin{array}{rrrrrr}10 & 6 & 12 & 61 & 40 & -22 \\ 11 & 6 & 12 & 21.4 & -22 & -14\end{array}$

$\begin{array}{llllll}12 & 6 & 12 & 31 & 19 & -42 \\ 13 & 6 & 12 & 59 & 10 & -27\end{array}$

$\begin{array}{llllll}13 & 7 & 12 & 59 & 10 & -27 \\ -6 & 72 & 26 & -50\end{array}$

$\begin{array}{llllll}-5 & 7 & 12 & 65 & 7 & -25\end{array}$

$\begin{array}{rrrrrr}-4 & 7 & 12 & 66 & -60 & -26 \\ -3 & 7 & 12 & 73 & 7 & -23\end{array}$

$\begin{array}{llllll}-2 & 7 & 12 & 40 & 14 & -25\end{array}$

$\begin{array}{llllll}-1 & 712 & 4 & 72 & -60 & -28\end{array}$

- 712

2712

3712

$\begin{array}{rrrrrr}4 & 7 & 12 & 99 & 87 & 11 \\ 5 & 7 & 12 & 97 & -91 & 11\end{array}$

$44 \quad 58-26$

$63-58-14$

$6 \quad 712 \quad 144 \quad 15513$

$\begin{array}{llllll}7 & 7 & 12 & 67 & -29 & -19\end{array}$

$\begin{array}{rrrrrr}9 & 7 & 12 & 105 & -83 & 13 \\ 1 & 7 & 12 & 43 & -9 & -25\end{array}$

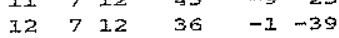

$\begin{array}{llllll}13 & 7 & 12 & 53 & -6 & -33\end{array}$

$\begin{array}{rrrrrr}-5 & 8 & 12 & 135 & -100 & 13 \\ -4 & 8 & 12 & 145 & 130 & 12\end{array}$

$\begin{array}{lllllll}-3 & 8 & 12 & 136 & -1.47 \quad 12\end{array}$

$\begin{array}{lllll}-2 & 8 & 12 & 194 & 185\end{array}$

$\begin{array}{llllll}-1 & 8 & \pm 2 & 217 & -219\end{array}$

$\begin{array}{rrrrr}0 & 8 & 12 & 308 & 297 \\ 1 & 8 & 12 & 317 & -317\end{array}$

$\begin{array}{llllll}2 & 8 & 12 & 23 & 43 & -36\end{array}$

$\begin{array}{llllll}3 & 12 & 44 & -8 & -25\end{array}$

$\begin{array}{rrrrrr}4 & 8 & 12 & 234 & 243 & 7 \\ 5 & 8 & 12 & 212 & -220 & 8\end{array}$

$\begin{array}{rrrrrr}5 & 8 & 12 & 212 & -220 & 8 \\ 6 & 8 & 12 & 202 & 202 & 8 \\ 7 & 8 & 12 & 138 & -113 & 11\end{array}$

$\begin{array}{rrrrrr}7 & 8 & 12 & 138 & -1.13 & 11 \\ 8 & 8 & 12 & 170 & 171 & 10\end{array}$

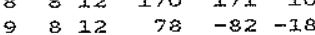

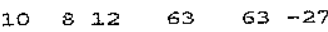

$\begin{array}{rrrrrr}11 & 8 & 12 & 54 & -18 & -31 \\ 32 & 8 & 12 & 76 & -1 & -19\end{array}$

$13 \quad 8 \quad 12 \quad 68 \quad-14 \quad-30$

$\begin{array}{llllll}-4 & 9 & 12 & 50 & -15 & -32\end{array}$

$\begin{array}{llllll}-3 & 9 & 12 & 45 & -15 & -32\end{array}$

$\begin{array}{llllll}-2 & 9 & 12 & 59 & 24 & -21 \\ -1 & 9 & 12 & 68 & 39 & -24\end{array}$

- $912 \quad 52 \quad 39-28$

$\begin{array}{lllll}9 & 12 & 56 & 33 & -20 \\ 9 & 12 & 42 & 38 & -29\end{array}$

$\begin{array}{lllll}9 & 12 & 144 & -148\end{array}$

$\begin{array}{rrrrr}9 & 12 & 103 & 116 & 12 \\ 9 & 12 & 67 & 59 & -18\end{array}$

$\begin{array}{lllll}912 & 89 & 78 & 14\end{array}$

$\begin{array}{lllll}9 & 12 & 157 & -176 & 10\end{array}$

$\begin{array}{lllll}912 & 97 & 14\end{array}$

$\begin{array}{rrrrrr}9 & 9 & 12 & 114 & -77 & 14 \\ 0 & 9 & 12 & 83 & 67 & -18\end{array}$

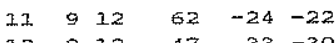

$\begin{array}{rrrrrr}12 & 9 & 12 & 47 & 33 & -30 \\ -3 & 10 & 12 & 62 & -76 & -29\end{array}$

$\begin{array}{llllll}-2 & 10 & 12 & 121 & 132 & 13\end{array}$

$\begin{array}{llllll}-1 & 30 & 12 & 288 & -166 & 10\end{array}$

- $1012 \quad 203 \quad 201$

$\begin{array}{lllll}10 & 12 & 1.82 & -163 & 9 \\ 2 & 10 & 12 & 191 & 203\end{array}$

$3 \begin{array}{lllll}30 & 12 & 134 & -146 & 11\end{array}$

$\begin{array}{rrrrrr}4 & 10 & 12 & 175 & 199 & 9 \\ 5 & 10 & 12 & 151 & -175 & 11\end{array}$

k 2 IOFO 1OFC $10 \mathrm{~S}$

$\begin{array}{llllll}6 & 10 & 12 & 177 & 190 & 10\end{array}$

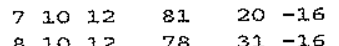

$9 \begin{array}{lllll}10 & 12 & 100 & -90 & 16\end{array}$

$101012 \quad 64 \quad 84-29$

$\begin{array}{rrrrrr}11 & 10 & 12 & 11 & -34 & -45 \\ 12 & 10 & 12 & 49 & 35 & -36\end{array}$

$\begin{array}{llllrr}-2 & 11 & 12 & 54 & 6 & -29\end{array}$

$\begin{array}{llllll}-1 & 11 & 12 & 24 & 26 & -47\end{array}$

$\begin{array}{rrrrrr}0 & 11 & 12 & 78 & 17 & -24 \\ 1 & 11 & 12 & 39 & 3 & -31\end{array}$

$\begin{array}{llllll}2 & 11 & 12 & 70 & -81 & -25 \\ & 12 & & \end{array}$

$\begin{array}{llllll}3 & 11 & 12 & 40 & -16 & -32\end{array}$

$\begin{array}{llllll}4 & 11 & 12 & 116 & 119 & 12\end{array}$

$\begin{array}{llllll}5 & 11 . & 12 & 98 & -112 & 14\end{array}$

$61112 \quad 49 \quad 27-29$

$\begin{array}{rrrrrr}7 & 11 & 12 & 68 & -204 & -19 \\ 8 & 11 & 12 & 49 & 67 & -31\end{array}$

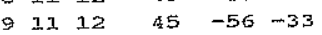

$\begin{array}{llllll}10 & 11 & 12 & 67 & -11 & -23\end{array}$

$\begin{array}{llllll}11 & 11 & 12 & 71 & -9 & -23\end{array}$

$\begin{array}{rrrrrr}-1 & 12 & 12 & 63 & -87 & -32 \\ 0 & 12 & 12 & 145 & 164 & 14\end{array}$

$\begin{array}{llllll}1 & 12 & 12 & 123 & -92 & 12\end{array}$

$\begin{array}{llllll}2 & 12 & 12 & 132 & 105 & 12\end{array}$

$\begin{array}{llllll}312 & 12 & 119 & -122 & 14\end{array}$

$\begin{array}{rrrrrr}4 & 12 & 12 & 86 & 88 & 16 \\ 5 & 12 & 12 & 55 & -69 & -28\end{array}$

$\begin{array}{llllll}6 & 12 & 12 & 127 & 162 & 14\end{array}$

$\begin{array}{llllll}7 & 12 & 12 & 139 & -111 & 12\end{array}$

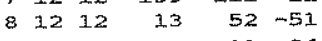

$\begin{array}{rrrrrr}9 & 12 & 12 & 61 & -31 & -26 \\ 10 & 12 & 12 & 56 & 42 & -27\end{array}$

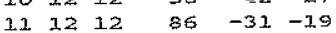

$\begin{array}{llllll}1 & 13 & 12 & 12 & 16 & -46\end{array}$

$\begin{array}{llllll}2 & 13 & 12 & 38 & 37 & -31\end{array}$

$\begin{array}{rrrrrr}3 & 13 & 12 & 53 & -17 & -24 \\ 4 & 13 & 12 & 47 & 28 & -31\end{array}$

$\begin{array}{llllll}13 & 12 & 48 & 20 & -33\end{array}$

$\begin{array}{llllll}7 & 13 & 12 & 49 & -17 & -33\end{array}$

$\begin{array}{lllllll}8 & 13 & 12 & 81 & 26 & -27\end{array}$

$\begin{array}{rrrrrr}9 & 13 & 12 & 84 & -38 & -19 \\ 4 & 14 & 12 & 88 & 99 & -19\end{array}$

$\begin{array}{llllll}5 & 14 & 12 & 63 & -62 & -21\end{array}$

$\begin{array}{lllllll}6 & 14 & 12 & 58 & 87 & -32\end{array}$

$\begin{array}{lllll}74 & 12 & 110 & -79 & 14\end{array}$

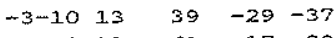

$\begin{array}{lllll}-2-10 & 13 & 61 & 17 & -28 \\ -1-10 & 13 & 40 & -2 & -40\end{array}$

$\begin{array}{lllll}0-10 & 13 & 57 & 3 & -30\end{array}$

$\begin{array}{lllll}1-10 & 13 & 13 & 10 & -51\end{array}$

$\begin{array}{rrrrrr}-5 & -9 & 13 & 96 & -79 & 17 \\ -4 & -9 & 13 & 95 & 91 & 16\end{array}$

$\begin{array}{llllll}-3 & -9 & 13 & 83 & -83 & -17\end{array}$

$\begin{array}{llllll}-1 & -9 & 13 & 38 & -85 & -37\end{array}$

$\begin{array}{llllll}0 & -9 & 13 & 88 & 108 & -18\end{array}$

$\begin{array}{rrrrrr}1 & -9 & 13 & 110 & -85 & 14 \\ 2 & -9 & 13 & 102 & 74 & 15\end{array}$

$\begin{array}{llllll}3 & -9 & 13 & 96 & -93 & 17\end{array}$

$\begin{array}{lllll}4-9 & 13 & 101 & 105 & 19\end{array}$

$\begin{array}{llllll}-6 & -8 & 13 & 79 & 18 & -25\end{array}$

$\begin{array}{rrrrrr}-5 & -8 & 13 & 59 & -25 & -27 \\ -4 & -8 & 13 & 60 & 16 & -27\end{array}$

$\begin{array}{llllll}-3 & -8 & 13 & 46 & -41 & -31\end{array}$

$\begin{array}{rrrrrr}-2 & -8 & 13 & 76 & 81 & -18 \\ -1 & -8 & 13 & 42 & -27 & -32\end{array}$

$\begin{array}{rrrrrr}-1 & -8 & 13 & 42 & -27 & -32 \\ 0 & -8 & 13 & 43 & -7 & -33\end{array}$

$\begin{array}{rrrrrr}1 & -8 & 13 & 28 & 28 & -42 \\ 2 & -8 & 13 & 48 & 1 . & -30\end{array}$

$\begin{array}{rrrrrr}2 & -8 & 13 & 48 & \text {.. } & -30 \\ 3 & -8 & 13 & 52 & -36 & -32\end{array}$

$\begin{array}{llllll}4 & -8 & 13 & 53 & -11 & -27\end{array}$

$\begin{array}{llllll}5 & -8 & 13 & 66 & 42 & -28\end{array}$

$\begin{array}{lllllll}-7 & -7 & 13 & 36 & -32 & -40 \\ -6 & -7 & 13 & 51 & 80 & -27\end{array}$

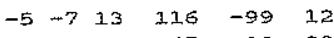

$\begin{array}{llllll}-4 & -7 & 13 & 67 & 89 & -20\end{array}$

$\begin{array}{llllll}-3 & -7 & 13 & 14.1 & -148 & 12\end{array}$

$\begin{array}{llllll}-2 & -7 & 13 & 143 & 147 & 2\end{array}$

$\begin{array}{rrrrrr}-1 & -7 & 13 & 155 & -109 & 9 \\ 0 & -7 & 13 & 188 & 177 & 9\end{array}$

$\begin{array}{llllll}1 . & -7 & 13 & 155 & -167 & 11\end{array}$

$\begin{array}{llllll}2 & -7 & 13 & 123 & 138 & 13\end{array}$

$\begin{array}{llllll}3 & -7 & 23 & 111 & -1.13 & 13\end{array}$

$\begin{array}{rrrrrr}4 & -7 & 13 & 111 & 103 & 14 \\ 5 & -7 & 13 & 87 & -116 & -20\end{array}$

$\begin{array}{llllll}6 & -7 & 13 & 105 & 121 & 15\end{array}$

$\begin{array}{llllll}7 & -7 & 13 & 93 & -64 & 18\end{array}$

$\begin{array}{rrrrrr}-8 & -6 & 13 & 51 & 22 & -37 \\ -7 & -6 & 13 & 65 & -39 & -28\end{array}$ $\mathrm{k} 1$ 2OFO IOFC LOS

$\begin{array}{llllll}-5 & -6 & 13 & 32 & -18 & -37\end{array}$

$\begin{array}{lllll}-6 & 13 & 35 & 23 & -36\end{array}$

$\begin{array}{llllll}-3 & -6 & 13 & 50 & -74 & -28\end{array}$

$\begin{array}{llllll}-2 & -6 & 13 & 61 & 79 & -20\end{array}$

$\begin{array}{lllllll}-1 & -6 & 13 & 25 & -68 & -41\end{array}$

$\begin{array}{llllll}0 & -6 & 13 & 21 & 68 & -41\end{array}$

$\begin{array}{rrrrrr}1 & -6 & 13 & 51 & 20 & -27\end{array}$

$\begin{array}{llllll}3 & -6 & 13 & 11 & -36 & -43\end{array}$

$\begin{array}{llllll}4 & -6 & 13 & 51 & 27 & -23\end{array}$

$\begin{array}{llllll}5 & -6 & 13 & 50 & 32 & -30\end{array}$

$\begin{array}{llllll}6 & -6 & 33 & 49 & -18 & -32\end{array}$

$\begin{array}{llllll}7 & -6 & 13 & 51 & 0 & -35\end{array}$

$\begin{array}{rrrrrr}8 & -6 & 13 & 53 & -1 & -36 \\ -8 & -5 & 13 & 62 & 48 & -29\end{array}$

$\begin{array}{llllll}-7 & -5 & 13 & 109 & -87 & 15\end{array}$

$\begin{array}{llllll}-6 & -5 & 13 & 1.3 & 138 & 15\end{array}$

$\begin{array}{llllll}-5 & -5 & 13 & 142 & -133 & 12\end{array}$

$\begin{array}{rrrrrr}4 & -5 & 13 & 114 & 117 & 13\end{array}$

$\begin{array}{llllll}-2 & -5 & 13 & 136 & 138 & 10\end{array}$

$\begin{array}{llllll}-1 & -5 & 13 & 153 & -157 & 10\end{array}$

$\begin{array}{rrrrrr}0 & -5 & 13 & 187 & 206 & 8 \\ 1 & -5 & 13 & 200 & -205 & 8\end{array}$

$\begin{array}{llllll}2 & -5 & 13 & 295 & 289 & 7\end{array}$

$\begin{array}{rrrrrr}3 & -5 & 13 & 283 & -281 & 7 \\ 1 & -5 & 13 & 210 & 182 & 8\end{array}$

$\begin{array}{rrrrrr}4 & -5 & 13 & 210 & 1.82 & 8 \\ 5 & -5 & 13 & 127 & -136 & 13\end{array}$

$\begin{array}{llllll}6 & -5 & 13 & 148 & 159 & 12\end{array}$

$\begin{array}{llllll}7 & -5 & 13 & 111 & -96 & 15\end{array}$

$\begin{array}{lllllll}8 & -5 & 13 & 44 & 49 & -36\end{array}$

$\begin{array}{llllll}9 & -5 & 13 & 77 & -38 & -20\end{array}$

$\begin{array}{llllll}-8 & -4 & 13 & 84 & -17 & -19 \\ -7 & -4 & 33 & 74 & -23 & -19\end{array}$

$\begin{array}{llllll}-6 & -4 & 13 & 51 & 69 & -27\end{array}$

$\begin{array}{llllll}-5 & -4 & 13 & 46 & -70 & -29\end{array}$

$\begin{array}{llllll}-4 & -4 & 13 & 91 & 97 & 15\end{array}$

$\begin{array}{llllll}-3 & -4 & 13 & 128 & -129 & 10\end{array}$

$\begin{array}{rrrrrr}-2 & -4 & 13 & 108 & 207 & 11 \\ -1 & -4 & 13 & 48 & 31 & -20\end{array}$

$\begin{array}{llllll}0 & -4 & 13 & 54 & -70 & -20\end{array}$

$\begin{array}{llllll}1 & -4 & 13 & 36 & 40 & -32\end{array}$

$\begin{array}{llllll}3 & -4 & 13 & 12 & 10 & -47\end{array}$

$\begin{array}{rrrrrr}4 & -4 & 13 & 74 & -22 & -16 \\ 5 & -4 & 13 & 87 & -62 & 15\end{array}$

$\begin{array}{llllll}6 & -4 & 13 & 65 & 27 & -20\end{array}$

$\begin{array}{llllll}7 & -4 & 13 & 78 & -25 & -18\end{array}$

$8-413 \quad 65 \quad 13-21$

$\begin{array}{rrrrrr}9 & -4 & 13 & 104 & 6 & -21 \\ -8 & -3 & 13 & 81 & 25 & -18\end{array}$

$\begin{array}{llllll}-7 & -3 & 13 & 62 & -20 & -28\end{array}$

$\begin{array}{llllll}-6 & -3 & 13 & 79 & 50 & -16\end{array}$

$\begin{array}{rrrrrr}-5 & -3 & 13 & 66 & -54 & -24 \\ -4 & -3 & 13 & 125 & 133 & 11\end{array}$

$\begin{array}{lllll}-3 & -3 & 13 & 210 & -206\end{array}$

$\begin{array}{lllll}-2 & -3 & 13 & 1.92 & 204\end{array}$

$\begin{array}{rrrrrr}-1 & -3 & 13 & 147 & -154 & 8 \\ 0 & -3 & 13 & 157 & 1.6 & 7\end{array}$

$\begin{array}{lllllll}1 & -3 & 13 & 56 & -69 & -17\end{array}$

$\begin{array}{rrrrrr}2 & -3 & 13 & 108 & 94 & 11 \\ 3 & -3 & 13 & 150 & -152 & 9\end{array}$

$\begin{array}{llllll}4 & -3 & 13 & 306 & 309 & 7\end{array}$

$\begin{array}{llllll}5 & -3 & 23 & 234 & -209\end{array}$

$\begin{array}{llllll}6 & -3 & 13 & 179 & 174 & 10\end{array}$

$\begin{array}{rrrrrr}7 & -3 & 13 & 161 & -169 & 11 \\ 8 & -3 & 13 & 101 & 83 & 15\end{array}$

$\begin{array}{llllll}10 & -3 & 13 & 49 & 41 & -37\end{array}$

$\begin{array}{llllll}-7 & -2 & 13 & 29 & -23 & -40\end{array}$

$\begin{array}{llllll}-5 & -2 & 13 & 45 & 1 & -27\end{array}$

$\begin{array}{rrrrrr}-4 & -2 & 13 & 20 & -6 & -40 \\ -3 & -2 & 13 & 33 & 5 & -32\end{array}$

$\begin{array}{llllll}-2 & -2 & 13 & 60 & 71 & -22\end{array}$

$\begin{array}{llllll}-1 & -2 & 13 & 111 & -94 & 16\end{array}$

$\begin{array}{rrrrrr}0 & -2 & 13 & 165 & 3 & -12 \\ 1 & -2 & 13 & 58 & 80 & -18\end{array}$

$\begin{array}{rrrrrr}1 & -2 & 13 & 58 & 80 & -18 \\ z & -2 & 13 & 102 & -97 & 9\end{array}$

$\begin{array}{lllllll}3 & -2 & 13 & 65 & 46 & -13\end{array}$

$\begin{array}{llllll}4 & -2 & 13 & 184 & -203 & 8\end{array}$

$\begin{array}{lllll}5 & -2 & 13 & 205 & 195\end{array}$

$\begin{array}{llllll}6 & -2 & 13 & 72 & 54 & -16\end{array}$

$\begin{array}{rrrrrr}7 & -2 & 13 & 49 & 2 & -30 \\ 8 & -2 & 13 & 51 & -19 & -31\end{array}$

$\begin{array}{llllll}9 & -2 & 13 & 29 & 7 & -38\end{array}$

$\begin{array}{llllll}10 & -2 & 13 & 80 & -45 & -19\end{array}$

$\begin{array}{llllll}-8 & -2 & 13 & 11 & -23 & -45\end{array}$

$\begin{array}{llllll}-7 & -1 & 13 & 50 & -21 & -29 \\ -6 & -1 & 13 & 49 & 75 & -25\end{array}$

$\begin{array}{llllll}-5 & -1 & 13 & 133 & -121 & 11\end{array}$

$\begin{array}{llllll}-4 & -1 & 13 & 150 & 157 & 10\end{array}$

$k 2$ LOFO IOFO $20 \mathrm{~S}$

$\begin{array}{rrrrrr}-2 & -1 & 13 & 237 & 249 & 7 \\ -1 & -1 & 13 & 172 & -180 & 7 \\ 0 & -1 & 13 & 229 & 239 & 6 \\ 1 & -1 & 13 & 238 & -233 & 6 \\ 2 & -1 & 13 & 253 & 261 & 6 \\ 3 & -1 & 13 & 175 & -155 & 7 \\ 4 & -1 & 13 & 123 & 103 & 9 \\ 5 & -1 & 13 & 297 & -298 & 6 \\ 6 & -1 & 13 & 218 & 228 & 8 \\ 7 & -1 & 13 & 121 & -99 & 11 \\ 8 & -1 & 13 & 157 & 147 & 11\end{array}$

$\begin{array}{rrrrrr}8 & -1 & 13 & 157 & 147 & 11 \\ 9 & -1 & 13 & 52 & -110 & -32\end{array}$

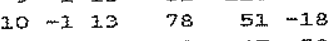

$\begin{array}{llllll}11 & -1 & 13 & 12 & -47 & -50 \\ -1 & 0 & 13 & 23 & -12 & -33\end{array}$

$\begin{array}{llllll}-7 & 0 & 13 & 51 & -3 & -21\end{array}$

$\begin{array}{llllll}-6 & 0 & 13 & 54 & -7 & -21\end{array}$ 


\begin{tabular}{|c|c|c|c|c|c|c|c|c|}
\hline 6 & 3 & 13 & 291 & 287 & 6 & -1 & 8 & 13 \\
\hline 7 & 3 & 13 & 228 & -237 & 7 & 0 & 8 & 13 \\
\hline 8 & 3 & 13 & 119 & 76 & 11 & 1 & 8 & 13 \\
\hline 9 & 3 & 13 & 95 & -90 & 14 & 2 & 8 & 13 \\
\hline 10 & 3 & 13 & 60 & 97 & -25 & 3 & 8 & 13 \\
\hline 11 & 3 & 13 & 70 & -34 & -27 & 4 & 8 & 13 \\
\hline 12 & 3 & 13 & 31 & 57 & -45 & 5 & 8 & 13 \\
\hline 13 & 3 & 13 & 13 & -17 & -53 & 6 & 8 & 13 \\
\hline-7 & 4 & 23 & 47 & 21 & -31 & 7 & 8 & 13 \\
\hline-6 & 4 & 13 & 34 & -2 & -40 & 8 & 8 & 13 \\
\hline-5 & 4 & 13 & 91 & -36 & 2.5 & 9 & 8 & 23 \\
\hline-4 & 4 & 13 & 61 & $2 d$ & -19 & 10 & 8 & 13 \\
\hline-3 & 4 & 13 & 91 & -73 & 14 & 11 & 8 & 13 \\
\hline-2 & 4 & 3.3 & 84 & 12 & -18 & 12 & 8 & 13 \\
\hline 0 & 4 & 13 & 316 & 119 & 8 & 13 & 8 & 13 \\
\hline 1 & 4 & 3.3 & 42 & 70 & -28 & -4 & 9 & 13 \\
\hline 2 & 4 & 13 & 189 & -181 & 7 & -3 & 9 & 13 \\
\hline 3 & 4 & 13 & 40 & 88 & -28 & -2 & 9 & 13 \\
\hline 4 & 4 & 13 & 61 & 22 & - \pm 5 & -1 & 9 & 13 \\
\hline 5 & 4 & 13 & 122 & -234 & 9 & 0 & 9 & 13 \\
\hline 6 & 4 & 13 & 276 & 181 & 8 & 1 & 9 & 13 \\
\hline 7 & 4 & 13 & 145 & -117 & 9 & 2 & 9 & 13 \\
\hline 8 & 4 & 13 & 29 & 21 & -38 & 3 & 9 & 23 \\
\hline 9 & 4 & 13 & 86 & -69 & 16 & 4 & 9 & 13 \\
\hline 10 & 4 & 13 & 60 & 38 & -28 & 5 & 9 & 13 \\
\hline 11 & 4 & 13 & 45 & -33 & -35 & 6 & 9 & 13 \\
\hline 12 & 4 & 13 & 62 & 24 & -23 & 7 & 9 & 13 \\
\hline 13 & 4 & 13 & 37 & 3.1 & -46 & 8 & 9 & 13 \\
\hline-7 & 5 & 13 & 26 & -5 & -42 & 9 & 9 & 33 \\
\hline-6 & 5 & 13 & 101 & 98 & 3.5 & 10 & 9 & 13 \\
\hline-5 & 5 & 33 & 106 & -91 & 14 & 11 & 9 & 13 \\
\hline-4 & 5 & 13 & 116 & 222 & 13 & 12 & 9 & 13 \\
\hline-3 & 5 & 23 & 236 & -214 & 8 & -3 & 10 & 33 \\
\hline-2 & 5 & 13 & 261 & 242 & 7 & -2 & 10 & 13 \\
\hline-1 & 5 & 13 & 292 & -298 & 7 & -1 & 10 & 3.3 \\
\hline 0 & 5 & 13 & 205 & $22 \%$ & 7 & 0 & 20 & 13 \\
\hline 1 & 5 & 13 & 156 & -172 & 7 & 1 & 10 & 13 \\
\hline 2 & 5 & 13 & 175 & 175 & 古 & 2 & 10 & 13 \\
\hline 3 & 5 & 2.3 & 170 & -168 & 7 & 3 & 10 & 13 \\
\hline 4 & 5 & 13 & 75 & 52 & 12 & 4 & 10 & 13 \\
\hline 5 & 5 & 13 & 106 & -94 & 10 & 5 & 10 & 13 \\
\hline 6 & 5 & 13 & 219 & 221 & 7 & 6 & 10 & 13 \\
\hline 7 & 5 & 13 & 236 & -247 & 7 & 7 & 10 & 13 \\
\hline 8 & 5 & 13 & 136 & 120 & 11 & 8 & 10 & 13 \\
\hline 9 & 5 & 13 & 44 & -77 & -29 & 9 & 10 & 13 \\
\hline 10 & 5 & 23 & 41 & 56 & -28 & 10 & 10 & 13 \\
\hline 12 & 5 & 13 & 58 & 15 & -25 & 1.1 & 10 & 13 \\
\hline 13 & 5 & 13 & 37 & -31 & -42 & 12 & 10 & 13 \\
\hline-6 & 6 & 13 & 36 & 28 & -40 & -2 & 11 & 13 \\
\hline-5 & 6 & 13 & 62 & 19 & -19 & -1 & 11 & 13 \\
\hline-4 & 6 & 13 & 55 & 16 & -21. & 0 & 11 & 13 \\
\hline-3 & 6 & 13 & 72 & 40 & -17 & 1 & 11 & 13 \\
\hline-2 & 6 & 13 & 57 & -62 & -25 & 2 & 21 & 23 \\
\hline-1 & 6 & 33 & 148 & 157 & 10 & 3 & 11. & 23 \\
\hline 0 & 6 & 13 & 96 & -107 & 32 & 4 & 11 & 13 \\
\hline 1 & 6 & 13 & 119 & -124 & 9 & 5 & 11 & 13 \\
\hline 2 & 6 & 13 & 65 & -23 & -20 & 6 & 11 & 13 \\
\hline 3 & 6 & 13 & 252 & 154 & 8 & 7 & 11 & 13 \\
\hline 4 & 6 & 13 & 89 & -103 & 12 & 8 & 11 & 13 \\
\hline 5 & 6 & 23 & 44 & -18 & -39 & 9 & 11 & 23 \\
\hline 6 & 6 & 13 & 117 & 125 & 10 & 10 & 11 & 2.3 \\
\hline 7 & 6 & 13 & 58 & -49 & -26 & 13 & 11 & 13 \\
\hline 8 & G & 13 & 56 & 10 & -20 & 0 & 12 & 13 \\
\hline 9 & 6 & 13 & 32 & -39 & -36 & 1 & 12 & 13 \\
\hline 10 & 6 & 13 & 43 & 50 & -26 & 2 & 12 & 13 \\
\hline 11 & 6 & 13 & 48 & -23 & -32 & 3 & 12 & 13 \\
\hline 12 & 6 & 13 & 54 & -14 & -27 & 4 & 12 & 13 \\
\hline 13 & 6 & 13 & 104 & 15 & 16 & 5 & 12 & 13 \\
\hline-5 & 7 & 13 & 96 & -86 & 16 & 6 & 12 & 13 \\
\hline-4 & 7 & 13 & 119 & 205 & 14 & 7 & 12 & 13 \\
\hline-3 & 7 & 13 & 133 & -147 & 12 & 8 & 12 & 13 \\
\hline-2 & 7 & 13 & 282 & 266 & 7 & 9 & 12 & 13 \\
\hline-1 & 7 & 13 & 192 & -282 & 9 & 10 & 12 & 13 \\
\hline 0 & 7 & 13 & 366 & 359 & 6 & 2 & 13 & 13 \\
\hline 1 & 7 & 13 & 165 & -159 & 9 & 3 & 13 & 13 \\
\hline 2 & 7 & 13 & 36 & -62 & -24 & 4 & 13 & 23 \\
\hline 3 & 7 & 13 & 32 & -38 & -30 & 5 & 13 & 13 \\
\hline 4 & 7 & 13 & 44 & 50 & -28 & 6 & 13 & 13 \\
\hline 5 & 7 & 13 & 42 & 19 & -26 & 7 & 13 & 13 \\
\hline 6 & 7 & 13 & 34 & -27 & -32 & 8 & 13 & 13 \\
\hline 7 & 7 & 1.3 & 119 & -98 & 12 & 9 & 13 & 13 \\
\hline 8 & 7 & 13 & 76 & 89 & -17 & -4 & -9 & 14 \\
\hline 9 & 7 & 1.3 & 138 & -113 & 1.2 & -3 & -9 & 24 \\
\hline 10 & 7 & 13 & 79 & 112 & -19 & -2 & -9 & 14 \\
\hline 13 & 7 & 13 & 45 & -50 & -33 & -1 & -9 & 14 \\
\hline 12 & 7 & 13 & 13 & 14 & -53 & 0 & -9 & 14 \\
\hline-5 & 8 & 13 & 60 & -2 & -29 & 1 & -9 & 14 \\
\hline-4 & 8 & 13 & 30 & -2 & -43 & 2 & $\sim 9$ & 14 \\
\hline-3 & 8 & 13 & 33 & -44 & -40 & -5 & -8 & 14 \\
\hline & 8 & & & & & -4 & -8 & 1.4 \\
\hline
\end{tabular}

$\begin{array}{llllll}-6 & -2 & 14 & 28 & -5 & -41\end{array}$

$\begin{array}{rrrrrr}-5 & -2 & 14 & 22 & -50 & -40 \\ -4 & -2 & 14 & 52 & 30 & -26\end{array}$

$\begin{array}{llllll}-3 & -2 & 14 & 116 & -117 & 11\end{array}$

$\begin{array}{llllll}-2 & -2 & 14 & 226 & 198 & 8 \\ -1 & -2 & 14 & 249 & -253 & 7\end{array}$

$\begin{array}{rrrrr}-1 & -2 & 14 & 249 & -253 \\ 0 & -2 & 14 & 354 & 366\end{array}$

$\begin{array}{rrrrrr}1 & -2 & 14 & 21.6 & -233 & 7\end{array}$

$\begin{array}{rrrrrr}2 & -2 & 14 & 99 & 120 & 11 \\ 3 & -2 & 14 & 69 & -38 & -14\end{array}$

$\begin{array}{rrrrrr}3 & -2 & 14 & 69 & -38 & -14 \\ 4 & -2 & 14 & 159 & 155 & 9\end{array}$

$\begin{array}{rrrrrr}5 & -2 & 14 & 171 & -171 & 9 \\ 6 & -2 & 14 & 132 & 128 & 1.1\end{array}$

$\begin{array}{rrrrrr}6 & -2 & 14 & 132 & 128 & 1.1 \\ 7 & -2 & 14 & 146 & -160 & 1.1\end{array}$

$\begin{array}{lllllll}8 & -2 & 14 & 141 & 151 & 12\end{array}$

$\begin{array}{llllll}9 & -2 & 14 & 67 & -94 & -30\end{array}$

$\begin{array}{llllll}10 & -2 & 14 & 110 & 75 & 16\end{array}$

$\begin{array}{llllrl}-8 & -1 & 14 & 12 & -1 & -48 \\ -7 & -1 & 14 & 84 & 5 & -21\end{array}$

$\begin{array}{rrrrrr}-6 & -1 & 14 & 94 & 5 & -21 \\ -18 & -20\end{array}$

$\begin{array}{lllllll}-5 & -1 & 14 & 78 & -50 & -17\end{array}$

$\begin{array}{rrrrrr}-4 & -1 & 14 & 36 & 4 & -33 \\ -3 & -1 & 14 & 24 & 28 & -38\end{array}$

$\begin{array}{rrrrrr}-3 & -1 & 14 & 24 & 28 & -38 \\ -2 & -1 & 14 & 150 & -159 & 10\end{array}$

$\begin{array}{lllllll}-1 & -1 & 14 & 72 & 98 & -15\end{array}$

$\begin{array}{llllll}0 & -1 & 14 & 179 & 19 & -12\end{array}$

$\begin{array}{llllll}1 & -1 & 14 & 77 & 10 & -18\end{array}$

$\begin{array}{lllllll}2 & -1 & 1.4 & 47 & -19 & -23\end{array}$

$\begin{array}{rrrrrr}3 & -1 & 14 & 11 & 46 & -43 \\ 4 & -1 & 14 & 89 & -110 & 31\end{array}$

$\begin{array}{rrrrrr}4 & -1 & 14 & 89 & -110 & 11 \\ 5 & -1 & 14 & 42 & 93 & -31\end{array}$

$\begin{array}{rrrrrr}5 & -1 & 14 & 42 & 93 & -31 \\ 6 & -1 & 14 & 53 & -47 & -27\end{array}$

$\begin{array}{rrrrrr}7 & -1 & 14 & 71 & 9 & -17 \\ 8 & -7 & 14 & 76 & -46 & -19\end{array}$

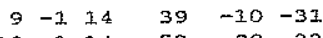

$\begin{array}{llllll}10 & -1 & 14 & 53 & -20 & -33\end{array}$

$\begin{array}{rrrrrr}11 & -1 & 14 & 52 & 7 & -32 \\ -8 & 0 & 14 & 83 & 37 & 14\end{array}$

$\begin{array}{rrrrrr}-8 & 0 & 14 & 83 & 37 & 14 \\ -7 & 0 & 14 & 63 & -15 & -19 \\ -6 & 0 & 14 & 45 & 60 & -23\end{array}$

$\begin{array}{llllll}-5 & 0 & 3.4 & 100 & -991 & 12\end{array}$

$\begin{array}{rrrrrr}-4 & 0 & 14 & 338 & 137 & 9 \\ -3 & 0 & 14 & 100 & -97 & 18\end{array}$

$\begin{array}{lllll}-2 & 0 & 14 & 164 & 151\end{array}$

$\begin{array}{lllll}-1 & 0 & 14 & 207 & -216\end{array}$

$\begin{array}{lllll}0 & 0 & 14 & 283 & 281\end{array}$

$\begin{array}{llllr}1 & 0 & 14 & 282 & -290 \\ 2 & 0 & 14 & 235 & 239\end{array}$

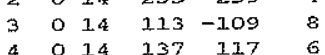

$\begin{array}{rrrrrr}4 & 0 & 14 & 137 & 117 & 6 \\ 5 & 0 & 14 & 35 & -39 & -23\end{array}$

$\begin{array}{rrrrrr}5 & 0 & 14 & 35 & -39 & -23 \\ 6 & 0 & 14 & 95 & 61 & -21\end{array}$

$7014 \quad 81-6512$

$\begin{array}{rrrrrr}8 & 0 & 14 & 82 & 100 & -18 \\ 9 & 0 & 14 & 115 & -121 & 10\end{array}$

$\begin{array}{rlrrrr}9 & 0 & 14 & 115 & -121 & 10 \\ 10 & 0 & 14 & 77 & 67 & -18\end{array}$

$11014 \quad 85 \quad-75 \quad 14$

$\begin{array}{llllll}-8 & 1 & 14 & 49 & -16 & -32\end{array}$

$\begin{array}{rrrrrr}-7 & 1 & 14 & 55 & 20 & -29 \\ -6 & 3 & 34 & 68 & 6 & -18\end{array}$

$\begin{array}{rrrrrr}-6 & 1 & 14 & 68 & 6 & -18 \\ -5 & 1 & 14 & 52 & 15 & -26\end{array}$

$\begin{array}{lllllll}-4 & 1 & 14 & 75 & -43 & -20\end{array}$

$\begin{array}{rrrrrr}-3 & 1 & 14 & 54 & 44 & -20 \\ -2 & 1 & 14 & 143 & 144 & 10\end{array}$

$\begin{array}{rrrrrr}-2 & 1 & 14 & 143 & 144 & 10 \\ -1 & 1 & 14 & 11 & -37 & -46\end{array}$

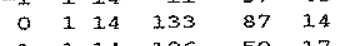

$\begin{array}{llllll}2 & 1 & 14 & 106 & 50 & 17\end{array}$

$\begin{array}{rrrrrr}2 & 1 & 14 & 177 & -168 & 7 \\ 3 & 1 & 14 & 88 & -94 & 11\end{array}$

$\begin{array}{rrrrrr}4 & 1 & 14 & 62 & 35 & -20 \\ 5 & 1 & 14 & 60 & 34 & -17\end{array}$

$\begin{array}{lllllll}6 & 1 & 14 & 58 & -37 & -23\end{array}$

$\begin{array}{lllllll}8 & 1 & 14 & 74 & -31 & -17\end{array}$

$\begin{array}{llllll}9 & 1 & 14 & 36 & -43 & -38\end{array}$

$\begin{array}{lllllll}10 & 1 & 14 & 24 & 30 & -46\end{array}$

$\begin{array}{rrrrrr}11 & 1 & 14 & 31 & 5 & -42 \\ 12 & 1 & 14 & 79 & 14 & -28\end{array}$

$\begin{array}{llllll}12 & 1 & 14 & 79 & 14 & -28 \\ -7 & 2 & 14 & 35 & -63 & -40\end{array}$

$\begin{array}{llllll}-6 & 2 & 14 & 108 & 100 & 16\end{array}$

$\begin{array}{rrrrrr}-5 & 2 & 14 & 150 & -1.44 & 11 \\ -4 & 2 & 14 & 132 & 103 & 11\end{array}$

$\begin{array}{llllll}-4 & 2 & 14 & 132 & 103 & 11 \\ -3 & 2 & 14 & 137 & -151 & 13\end{array}$

$\begin{array}{rrrrrr}-3 & 2 & 14 & 137 & -151 & 11 \\ -2 & 2 & 24 & 132 & 93 & 9\end{array}$

$\begin{array}{rrrrrr}-2 & 2 & 14 & 132 & 93 & 9 \\ -1 & 2 & 14 & 32 & -35 & -31\end{array}$

$\begin{array}{lllllll}0 & 2 & 14 & 371 & 387 & 6\end{array}$

$\begin{array}{rrrrrrr}1 & 2 & 14 & 320 & -347 & 6 \\ 2 & 2 & 14 & 348 & 379 & 5\end{array}$

$3214 \quad 272$ m279 6

$\begin{array}{rrrrrr}4 & 2 & 14 & 92 & 89 & 31 \\ 5 & 2 & 14 & 176 & -1.96 & 7\end{array}$ $\begin{array}{llllll}6 & 2 & 14 & 104 & 118 & 12\end{array}$

$\begin{array}{llllll}7 & 2 & 14 & 106 & -83 & 13\end{array}$

$\begin{array}{rrrrrr}8 & 2 & 14 & 40 & 6 & -32 \\ 10 & 2 & 14 & 33 & 47 & -37\end{array}$

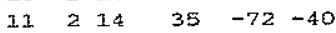

$\begin{array}{llllll}12 & 2 & 14 & 66 & 90 & -29\end{array}$

$\begin{array}{llllll}-7 & 3 & 14 & 25 & 30 & -48\end{array}$

$\begin{array}{rrrrrr}-6 & 3 & 14 & 42 & 44 & -33 \\ -5 & 3 & 14 & 84 & -39 & 16\end{array}$

$\begin{array}{lllllll}-4 & 3 & 14 & 38 & -13 & -35\end{array}$

$\begin{array}{llllll}-3 & 3 & 14 & 25 & -20 & -38\end{array}$

$\begin{array}{llllll}-2 & 3 & 14 & 37 & -77 & -32\end{array}$

$\begin{array}{llllll}0 & 3 & 14 & 103 & 107 & 10 \\ 1 & 3 & 14 & 86 & 34 & -38\end{array}$

$\begin{array}{rrrrrr}1 & 3 & 14 & 86 & 31 & -18 \\ 2 & 3 & 14 & 66 & -41 & -20\end{array}$

$\begin{array}{llllll}3 & 3 & 14 & 62 & -68 & -16\end{array}$

$\begin{array}{llllll}4 & 3 & 14 & 259 & 167 & 8\end{array}$

$\begin{array}{rrrrrr}5 & 3 & 14 & 29 & -13 & -34 \\ 6 & 3 & 14 & 70 & -5 & -15\end{array}$

$\begin{array}{llllll}7 & 3 & 14 & 49 & -49 & -29\end{array}$

$\begin{array}{rrrrrr}8 & 3 & 14 & 80 & 51 & 15 \\ 9 & 3 & 14 & 40 & -32 & -27\end{array}$

$\begin{array}{rrrrrr}9 & 3 & 14 & 40 & -32 & -27 \\ 10 & 3 & 14 & 28 & 1 & -37\end{array}$

$11 \quad 314 \quad 11 \quad 4 \quad-45$

$\begin{array}{rrrrrr}12 & 3 & 14 & 13 & 15 & -52 \\ -7 & 4 & 14 & 45 & -20 & -35\end{array}$

$\begin{array}{lllllll}-6 & 4 & 14 & 99 & 95 & 15\end{array}$

$\begin{array}{llllll}-5 & 4 & 14 & 105 & -98 & 15\end{array}$

$\begin{array}{llllll}-4 & 4 & 14 & 186 & 174 & 10\end{array}$

$\begin{array}{rrrrrr}-3 & 4 & 14 & 230 & -245 & 8 \\ -2 & 4 & 14 & 286 & 271 & 7\end{array}$

$\begin{array}{lllllll}-1 & 4 & 14 & 290 & -292 & 7\end{array}$

$\begin{array}{rrrrrr}\circ & 4 & 14 & 148 & 158 & 8 \\ 1 & 4 & 14 & 207 & -187 & 6\end{array}$

$\begin{array}{rrrrrr}2 & 4 & 14 & 174 & 184 & 7\end{array}$

$\begin{array}{rrrrrr}3 & 4 & 14 & 293 & -300 & 6 \\ 4 & 4 & 34 & 128 & 127 & 9\end{array}$

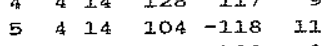

$\begin{array}{llllll}6 & 4 & 14 & 194 & 180 & 8\end{array}$

$\begin{array}{rrrrrr}7 & 4 & 14 & 171 & -159 & 9 \\ 8 & 4 & 34 & 71 & 62 & -18\end{array}$

$\begin{array}{rrrrrr}9 & 4 & 34 & 71 & 62 & -18 \\ 9 & 4 & 14 & 89 & -113 & 16\end{array}$

$10 \quad 4 \quad 14 \quad 96 \quad 88 \quad 16$

$\begin{array}{llllll}11 & 4 & 1.4 & 72 & -42 & -38\end{array}$

\begin{tabular}{llllrr}
12 & 4 & 14 & 60 & 36 & -31 \\
\hline & 4 & 14 & 54 & -29 & -38
\end{tabular}

$\begin{array}{rrrrrr}13 & 4 & 14 & 54 & -29 & -38 \\ -6 & 5 & 14 & 12 & 5 & -49\end{array}$

$\begin{array}{rrrrrr}-5 & 5 & 14 & 45 & 3 & -31 \\ -3 & 5 & 14 & 34 & -1 & -35\end{array}$

$\begin{array}{lllllr}-3 & 5 & 14 & 34 & -2 & -35 \\ -2 & 5 & 14 & 81 & 55 & 15\end{array}$

$\begin{array}{rrrrrr}-1 & 5 & 14 & 59 & 23 & -26\end{array}$

$\begin{array}{rrrrrr}0 & 5 & 14 & 47 & 109 & -29 \\ 1 & 5 & 14 & 97 & -119 & 11\end{array}$

$\begin{array}{llllll}2 & 5 & 14 & 53 & 42 & -23\end{array}$

$\begin{array}{rrrrrr}3 & 5 & 14 & 43 & -56 & -27 \\ 4 & 5 & 14 & 41 & 49 & -27\end{array}$

$6514 \quad 66 \quad-64-22$

$\begin{array}{llllll}7 & 5 & 14 & 36 & 49 & -29\end{array}$

$8 \begin{array}{lllll}8 & 14 & 71 & 44 & -18\end{array}$

$\begin{array}{rrrrrr}9 & 5 & 14 & 62 & -58 & -22 \\ 10 & 5 & 14 & 77 & 51 & -19\end{array}$

$\begin{array}{llllll}11 & 5 & 14 & 24 & 0 & -44 \\ 14 & 5 & 14 & 36 & 9 & -38\end{array}$

$\begin{array}{lllllll}13 & 5 & 14 & 46 & -12 & -39\end{array}$

$\begin{array}{llllll}-6 & 5 & 14 & 55 & 42 & -29\end{array}$

$\begin{array}{rrrrrr}-5 & 6 & 14 & 93 & -84 & 16\end{array}$

$\begin{array}{rrrrrr}-4 & 6 & 14 & 23 & 76 & -44 \\ -3 & 6 & 1.4 & 152 & -347 & 11\end{array}$

$\begin{array}{lllll}-2 & 6 & 14 & 222 & 193\end{array}$

$\begin{array}{rrrrr}-1 & 6 & 14 & 247 & -248 \\ 0 & 6 & 14 & 382 & 382\end{array}$

$\begin{array}{rrrrr}0 & 6 & 14 & 382 & 382 \\ 1 & 6 & 14 & 182 & -183\end{array}$

$\begin{array}{lllll}6 & 14 & 183 & 200\end{array}$

$\begin{array}{llllll}3 & 6 & 14 & 232 & -232 & 6\end{array}$

$\begin{array}{rrrrrr}4 & 6 & 14 & 231 & 233 & 6 \\ 5 & 6 & 14 & 91 & -89 & 11\end{array}$

$\begin{array}{rrrrrr}5 & 6 & 14 & 91 & -89 & 11 \\ 6 & 6 & 14 & 50 & 45 & -24\end{array}$

$\begin{array}{llllll}7 & 6 & 14 & 161 & -159 & 10\end{array}$

$\begin{array}{rrrrrrr}8 & 6 & 14 & 68 & 24 & -18 \\ 9 & 6 & 14 & 104 & -71 & 14\end{array}$

$\begin{array}{llllll}10 & 6 & 14 & 57 & 90 & -31\end{array}$

$\begin{array}{llllll}12 & 6 & 14 & 62 & 84 & -32\end{array}$

$\begin{array}{rrrrrr}13 & 6 & 14 & 29 & -21 & -40 \\ -5 & 7 & 14 & 93 & 14 & -22\end{array}$

$\begin{array}{llllll}-3 & 7 & 14 & 69 & 24 & -18\end{array}$

$\begin{array}{llllll}-2 & 7 & 14 & 65 & -5 & -24\end{array}$

$\begin{array}{rrrrrr}-1 & 7 & 14 & 37 & -25 & -31 \\ 0 & 7 & 14 & 38 & -15 & -32\end{array}$

$\begin{array}{rrrrrr}1 & 7 & 14 & 42 & 10 & -29\end{array}$

$\begin{array}{llllll}2 & 714 & 14 & 48 & -24\end{array}$ 
$\mathrm{h} \mathrm{k}$ I $10 \mathrm{FO} 10 \mathrm{FC}$ los

$\begin{array}{llllll}3 & 7 & 14 & 54 & -11 & -17\end{array}$

$\begin{array}{rrrrrr}4 & 7 & 14 & 12 & 5 & -47 \\ 5 & 7 & 14 & 53 & 30 & -24\end{array}$

$\begin{array}{llllll}6 & 7 & 14 & 90 & 67 & 13 \\ 7 & 7 & 14 & 80 & 28 & 15\end{array}$

$\begin{array}{llllll}8 & 7 & 14 & 78 & -65 & -16 \\ 9 & 7 & 14 & 54 & -11 & -22\end{array}$

$\begin{array}{llllll}9 & 7 & 14 & 54 & -11 & -22 \\ 10 & 7 & 14 & 57 & -36 & -30\end{array}$

$\begin{array}{llllll}11 & 7 & 14 & 73 & -2 & -25 \\ 12 & 7 & 14 & 36 & -8 & -42\end{array}$

$\begin{array}{rrrrrr}12 & 7 & 14 & 36 & -8 & -42 \\ 13 & 7 & 14 & 57 & -12 & -32 \\ -4 & 8 & 14 & 95 & 73 & 17\end{array}$

$\begin{array}{llllll}-3 & 8 & 14 & 101 & -102 & 15\end{array}$

$\begin{array}{rrrrrr}-2 & 8 & 14 & 141 & 137 & 11 \\ -1 & 8 & 14 & 104 & -115 & 14\end{array}$

$\begin{array}{rrrrrr}-1 & 8 & 14 & 104 & -115 & 14 \\ 0 & 8 & 14 & 214 & 205 & 8\end{array}$

$\begin{array}{rrrrrr}1 & 8 & 14 & 233 & -258 & 8 \\ 2 & 3 & 14 & 200 & 207 & 8\end{array}$

$\begin{array}{llllll}3 & 8 & 14 & 243 & -242 & 7\end{array}$

$\begin{array}{rrrrrr}4 & 8 & 14 & 132 & 118 & 10 \\ 5 & 8 & 14 & 125 & -104 & 10\end{array}$

$\begin{array}{rrrrrr}6 & 8 & 14 & 46 & 18 & -27 \\ 7 & 8 & 14 & 41 & -52 & -25\end{array}$

$\begin{array}{llllll}8 & 8 & 14 & 97 & 101 & 16\end{array}$

$\begin{array}{llllll}9 & 8 & 14 & 93 & -75 & 16\end{array}$

$\begin{array}{lllllll}10 & 8 & 14 & 58 & 74 & -29\end{array}$

$\begin{array}{rrrrrr}11 & 8 & 14 & 53 & -41 & -26 \\ 12 & 8 & 14 & 55 & 24 & -33\end{array}$

$\begin{array}{llllll}-3 & 9 & 14 & 82 & 45 & 16\end{array}$

$\begin{array}{llllll}-2 & 9 & 14 & 68 & -17 & -26\end{array}$

$\begin{array}{rrrrrr}-1 & 9 & 14 & 43 & -10 & -31 \\ 0 & 9 & 14 & 63 & 24 & -24\end{array}$

$\begin{array}{rrrrrr}1 & 9 & 14 & 30 & -21 & -37 \\ 2 & 9 & 14 & 42 & 50 & -30\end{array}$

$\begin{array}{lllllll}3 & 9 & 14 & 95 & -89 & 14\end{array}$

$\begin{array}{llllll}4 & 9 & 14 & 42 & 10 & -25\end{array}$

$59 \begin{array}{lllll}5 & 9 & 36 & 18 & -33\end{array}$

$\begin{array}{llllrr}6 & 9 & 14 & 72 & -105 & -17 \\ 7 & 9 & 14 & 48 & 31 & -28\end{array}$

$\begin{array}{llllll}8 & 9 & 14 & 27 & -57 & -42\end{array}$

$\begin{array}{rrrrrr}9 & 9 & 14 & 46 & 40 & -30 \\ 10 & 9 & 14 & 48 & 13 & -32\end{array}$

$\begin{array}{rrrrrr}11 & 9 & 14 & 107 & -12 & 21 \\ 12 & 9 & 14 & 32 & 38 & -43\end{array}$

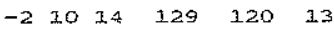

$\begin{array}{llllll}-1 & 10 & 14 & 160 & -135 & 13\end{array}$

$\begin{array}{llllll}0 & 10 & 14 & 127 & 135 & 13\end{array}$

$\begin{array}{llllll}1 & 10 & 14 & 149 & -157 & 10\end{array}$

$\begin{array}{rrrrrr}2 & 10 & 14 & 79 & 119 & -19 \\ 3 & 10 & 14 & 192 & -189 & 9\end{array}$

$\begin{array}{llllll}4 & 10 & 14 & 240 & 277 \quad 8\end{array}$

$\begin{array}{lllll}510 & 14 & 246 & -279 & 8\end{array}$

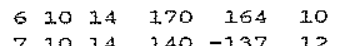

$\begin{array}{rrrrrr}7 & 10 & 14 & 140 & -137 & 12 \\ 8 & 10 & 14 & 74 & -16 & -23\end{array}$

$91014 \quad 24-48-45$

$\begin{array}{rrrrrr}10 & 10 & 14 & 65 & 44 & -26 \\ 11 & 10 & 14 & 96 & -41 & 18\end{array}$

$\begin{array}{rrrrrr}11 & 10 & 14 & 96 & -41 & 18 \\ 12 & 30 & 14 & 13 & 38 & -53\end{array}$

$\begin{array}{rrrrrr}-1 & 11 & 14 & 100 & 64 & 16\end{array}$

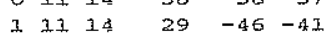

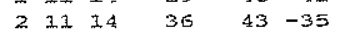

$\begin{array}{llllll}3 & 11 & 14 & 23 & -50 & -44 \\ 4 & 13 & 34 & 59 & 19 & -25\end{array}$

$\begin{array}{llllll}4 & 13 & 14 & 59 & 19 & -25 \\ 5 & 11 & 14 & 73 & 43 & -17\end{array}$

$6 \begin{array}{lllll}61 & 14 & 102 & 87 & 14\end{array}$

$\begin{array}{llllll}7 & 11 & 14 & 47 & -53 & -29\end{array}$

$81114 \quad 24 \quad 62-45$

$\begin{array}{llllll}9 & 11 & 14 & 57 & -32 & -29 \\ 10 & 31 & 14 & 25 & 2 & -48\end{array}$

$\begin{array}{rrrrrr}10 & 11 & 14 & 25 & 2 & -48 \\ 11 & 11 & 14 & 55 & -6 & -31\end{array}$

$\begin{array}{llllll}1 & 12 & 14 & 137 & -135 & 12\end{array}$

$\begin{array}{llllll}2 & 12 & 14 & 46 & 65 & -27\end{array}$

$\begin{array}{rrrrrr}3 & 12 & 14 & 102 & -115 & 14 \\ 4 & 12 & 14 & 78 & 90 & -19\end{array}$

$\begin{array}{lllllll}5 & 12 & 14 & 114 & -128 & 15\end{array}$

$\begin{array}{llllll}6 & 12 & 14 & 87 & 97 & 17\end{array}$

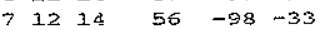

$8121430 \quad 101-48$

$\begin{array}{llllll}9 & 12 & 14 & 48 & -79 & -32 \\ 2 & 14 & 96 & 81 & -22\end{array}$

$\begin{array}{rrrrrr}10 & 12 & 14 & 76 & 81 & -22 \\ 3 & 13 & 14 & 35 & -48 & -38\end{array}$

$\begin{array}{llllrl}4 & 13 & 34 & 63 & 54 & -20\end{array}$

$\begin{array}{llllll}5 & 13 & 14 & 32 & -29 & -32\end{array}$

$\begin{array}{rrrrrr}6 & 13 & 14 & 49 & 25 & -33 \\ 7 & 13 & 14 & 95 & -14 & 16\end{array}$

8 $13 \begin{array}{llll}14 & 83 & -24 & -29\end{array}$

-1. $\begin{array}{lllll}-9 & 15 & 93 & -70 & 16\end{array}$

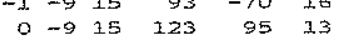

\begin{tabular}{|c|c|c|c|c|c|c|c|c|c|}
\hline-4 & $\begin{array}{lll}-3 & 15\end{array}$ & 15 & 32 & 31 & -49 & 2 & -215 & 5 & 44 \\
\hline-3 & $\begin{array}{lll}-8 & 1\end{array}$ & .5 & 52 & -48 & -33 & 3 & -21 & 5 & 10 \\
\hline-2 & $\begin{array}{lll}-8 & 1\end{array}$ & 15 & 58 & 44 & -23 & 4 & -219 & 15 & 54 \\
\hline-1 & -81 & 15 & 48 & -29 & -32 & 5 & -21 & 15 & 11 \\
\hline $\begin{array}{l}0 \\
1\end{array}$ & -81 & 15 & 88 & -1 & -23 & 6 & -21 & 5 & 46 \\
\hline$\frac{1}{2}$ & -81 & 15 & 67 & 15 & -19 & 7 & -21 & 5 & 72 \\
\hline $\begin{array}{l}2 \\
3\end{array}$ & -81 & 15 & 63 & \pm 7 & -27 & 8 & -21 & 5 & 79 \\
\hline $\begin{array}{l}3 \\
4\end{array}$ & -81 & 15 & 44 & -22 & -37 & 9 & -21 & 15 & 100 \\
\hline $\begin{array}{r}4 \\
-5\end{array}$ & -83 & 25 & 45 & -26 & -34 & 10 & -21 & 1.5 & 62 \\
\hline-5 & -71 & 15 & 43 & -38 & -34 & -7 & $\begin{array}{ll}-1 & 1\end{array}$ & 15 & 36 \\
\hline-3 & $\begin{array}{ll}-7 & 1 \\
-7\end{array}$ & 15 & 40 & 33 & -29 & -6 & $\begin{array}{lll}-1 & 1\end{array}$ & 15 & 63 \\
\hline-2 & $\begin{array}{ll}-7 & 1 \\
-7 & 1\end{array}$ & 15 & 66 & -54 & -20 & 5 & -32 & 5 & 96 \\
\hline-1 & $\begin{array}{ll}-7 & 1 \\
-7 & 1\end{array}$ & 15 & 33 & 61 & -39 & -4 & $\begin{array}{lll}-1 & 1\end{array}$ & 5 & 98 \\
\hline 0 & $\begin{array}{ll}-7 & 1 \\
-7 & 1\end{array}$ & $\begin{array}{l}15 \\
15\end{array}$ & $\begin{array}{l}120 \\
139\end{array}$ & $\begin{array}{l}-85 \\
136\end{array}$ & $\begin{array}{l}12 \\
13\end{array}$ & $\begin{array}{l}-3 \\
-2\end{array}$ & $\begin{array}{ll}-1 & 1 \\
-1 & 1\end{array}$ & $\begin{array}{l}15 \\
15\end{array}$ & $\begin{array}{l}104 \\
142\end{array}$ \\
\hline 1 & -71 & 15 & 218 & -199 & 9 & -1 & $\begin{array}{ll}-1 & 1\end{array}$ & 15 & 124 \\
\hline 2 & -7 & 15 & 202 & 172 & 10 & 0 & $\begin{array}{ll}-1 & 1\end{array}$ & 15 & 237 \\
\hline 3 & -71 & 15 & 118 & -96 & 1.4 & 1 & $\begin{array}{ll}-1 & 1\end{array}$ & 15 & 286 \\
\hline 4 & -73 & 1.5 & 47 & 53 & -35 & 2 & -13 & 15 & 154 \\
\hline 5 & -71 & 1.5 & 32 & -56 & -45 & 3 & $\begin{array}{ll}-1 & 1\end{array}$ & 15 & 229 \\
\hline-6 & -61 & 1.5 & 90 & 13 & -23 & 4 & $\begin{array}{ll}-1 & 1\end{array}$ & 25 & 127 \\
\hline-5 & -62 & 25 & 72 & -4 & -21 & 5 & $-1 \quad 1$ & 1.5 & 78 \\
\hline-4 & -61 & 15 & 12 & 19 & -50 & 6 & $\begin{array}{ll}-1 & 1\end{array}$ & 15 & 85 \\
\hline-3 & -61 & 15 & 23 & -47 & -42 & 7 & -11 & 15 & 44 \\
\hline-2 & -61 & 1.5 & 42 & 19 & -30 & 8 & $\begin{array}{ll}-1 & 1\end{array}$ & 15 & 98 \\
\hline-1 & -61 & 15 & 57 & -1 & -26 & 9 & $\begin{array}{ll}-1 & 1\end{array}$ & 1.5 & 59 \\
\hline 0 & -61 & 15 & 44 & 24 & -31 & Io & -11 & 15 & 67 \\
\hline 1 & -63 & 15 & 89 & -65 & 15 & 11 & -11 & 15 & 24 \\
\hline 2 & -61 & 15 & 39 & 29 & -35 & -7 & 01 & 15 & 47 \\
\hline 3 & -61 & 15 & 67 & -48 & -19 & -6 & 01 & 15 & 34 \\
\hline 4 & -61 & 15 & 69 & $2 \pi$ & -25 & -5 & 01 & 1.5 & 58 \\
\hline 5 & -61 & 15 & 25 & 18 & -45 & -4 & $\begin{array}{ll}0 & 1 .\end{array}$ & 1.5 & 87 \\
\hline 7 & -63 & 1.5 & 82 & 10 & -19 & -3 & 01 & 1.5 & 47 \\
\hline-5 & -51 & 15 & $6 d$ & 45 & -22 & -2 & 01 & 15 & 29 \\
\hline-5 & -51 & 15 & 80 & -68 & -18 & -1 & 03 & 25 & 53 \\
\hline-4 & -51 & 15 & 112 & 99 & 14 & $\circ$ & 01 & 15 & 168 \\
\hline-3 & -53 & 1.5 & 101 & -118 & 24 & 1 & 01 & 25 & 41 \\
\hline-2 & -51 & 15 & 111 & 103 & 13 & 2 & 01 & 15 & 72 \\
\hline-1 & -51 & 15 & 37 & -72 & -35 & 3 & 01 & 15 & 46 \\
\hline 0 & -51 & 15 & 37 & 92 & -34 & 4 & 01 & 15 & 82 \\
\hline 1 & -51 & 15 & 159 & -157 & 10 & 5 & 01 & 15 & 49 \\
\hline 2 & -51 & 15 & 172 & 167 & 10 & 6 & 01 & 15 & 65 \\
\hline 3 & -53 & 15 & 218 & -223 & 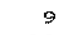 & 7 & 03 & 3.5 & 71 \\
\hline 4 & -53 & 15 & 181 & 150 & 10 & 8 & 01 & 15 & 11.4 \\
\hline 5 & -51 & 15 & 137 & -122 & 11 & 9 & 01 & 15 & 43 \\
\hline 6 & -53 & 15 & 121 & 238 & 15 & 10 & D I & 15 & 64 \\
\hline 7 & -53 & 1.5 & 65 & -84 & -31 & 11 & 01 & 15 & 65 \\
\hline 8 & -52 & 25 & 66 & 45 & -23 & -7 & 11 & 15 & 37 \\
\hline-7 & -43 & 15 & 63 & -22 & -29 & -6 & 11 & 15 & 150 \\
\hline-6 & -41 & 15 & 30 & 28 & -38 & -5 & 11 & 15 & 132 \\
\hline-5 & -41 & 15 & 44 & -33 & -34 & -4 & 11 & 15 & 121 \\
\hline-3 & $\begin{array}{ll}-4 & 1\end{array}$ & 15 & 1.1. & -76 & -43 & -3 & 13 & 15 & 195 \\
\hline-2 & -43 & 15 & 63 & 46 & -25 & -2 & 11 & 1.5 & 60 \\
\hline-1 & -41 & 15 & 29 & 24 & -36 & -1 & 11 & 1.5 & 154 \\
\hline 0 & -43 & 15 & 57 & 43 & -25 & 0 & 13 & 15 & 256 \\
\hline 1 & -41 & 15 & 41 & -81 & -32 & 1 & 11 & 25 & 236 \\
\hline 2 & -43 & 15 & so & 69 & -22 & 2 & 11 & 15 & 328 \\
\hline 3 & -42 & 25 & 12 & -35 & -47 & 3 & 11 & 15 & 46 \\
\hline 4 & -43 & 15 & 73 & -30 & -18 & 4 & 11 & 15 & 180 \\
\hline 5 & -43 & 15 & 48 & -12 & -29 & 5 & 11 & 15 & 1.82 \\
\hline 6 & -4 & 15 & 55 & 30 & -31 & 6 & I 1 & 15 & 120 \\
\hline 7 & -43 & 15 & 46 & -20 & -38 & 7 & 11 & 15 & 67 \\
\hline 8 & -43 & 15 & 65 & -14 & -31 & 8 & 13 & 1.5 & 44 \\
\hline 9 & $-4=$ & 15 & 33 & 31 & -44 & 9 & 13 & 3.5 & 70 \\
\hline-7 & -37 & 15 & 23 & -29 & -52 & 10 & 13 & 25 & 73 \\
\hline-6 & -33 & 15 & 54 & 60 & -29 & 11 & 11 & 15 & 25 \\
\hline-5 & -31 & 25 & 95 & -67 & 15 & 12 & & 15 & 5 \\
\hline-4 & -32 & 25 & 91 & 75 & 14 & -7 & 21 & 15 & 63 \\
\hline-3 & -37 & 25 & 181 & -192 & 10 & -6 & 21 & 15 & 84 \\
\hline-2 & -37 & 15 & 183 & 169 & 9 & -5 & 7 & 15 & 29 \\
\hline-1 & -31 & 15 & 233 & -221 & 7 & -4 & 23 & 15 & 27 \\
\hline o & -31 & 15 & 227 & 221 & 7 & -2 & 23 & 15 & 113 \\
\hline 2 & -31 & 15 & 169 & -169 & 8 & -3 & 2 & 15 & 20 \\
\hline 2 & -31 & 15 & 166 & 154 & 8 & 0 & 2 & 15 & 60 \\
\hline 3 & -37 & 15 & 149 & -153 & 20 & 1 & 2 & 15 & 143 \\
\hline 4 & -33 & 3.5 & 181 & 186 & 9 & 2 & 21 & 15 & 29 \\
\hline$s$ & -32 & 25 & 201 & -200 & 9 & 3 & 23 & 15 & 187 \\
\hline 6 & $-3=$ & 25 & 150 & 160 & 1. & 4 & 23 & 15 & 50 \\
\hline 7 & -3 & 15 & 130 & -127 & 13 & 5 & 21 & 15 & 55 \\
\hline 8 & $-3=$ & 15 & 88 & 67 & -19 & 6 & 2 & 15 & 55 \\
\hline & $-3=$ & 15 & 56 & -86 & -28 & 7 & 23 & 15 & \\
\hline & -23 & 15 & 59 & 0 & -28 & 9 & 23 & 15 & 28 \\
\hline & -2 & 15 & 34 & 9 & -40 & 10 & 2 & 15 & 82 \\
\hline & -23 & 15 & 49 & -10 & -29 & 12 & 2 & 15 & 59 \\
\hline-4 & -2 & 15 & 62 & 60 & -19 & 12 & 2 & 15 & 4 \\
\hline & -2 & 15 & 60 & -73 & -20 & -6 & 3 & 15 & 39 \\
\hline-2 & -2 & 15 & 22 & -9 & -33 & -5 & 3 & 15 & 126 \\
\hline-1 & -2 & 15 & 1.37 & -4 & -14 & -4 & 3 & 1.5 & 144 \\
\hline & & 15 & 176 & 14 & -13 & & & 15 & 195 \\
\hline & & & & & & & & & \\
\hline
\end{tabular}

$0 \quad 8 \quad 15$

$49 \quad 3-29$

$\begin{array}{lllll}2 & 85 & 63 & -34 & -25\end{array}$

$3 \quad 8 \quad 15 \quad 50 \quad 39-21$

$\begin{array}{llllll}4 & 8 & 15 & 58 & -29 & -27\end{array}$

$\begin{array}{llllll}6 & 8 & 15 & 47 & 16 & -29 \\ 7 & 8 & 15 & 54 & -3 & -22\end{array}$

$\begin{array}{rrrrrr}7 & 8 & 15 & 54 & -3 & -22 \\ 8 & 8 & 35 & 98 & -85 & 14\end{array}$

$\begin{array}{rrrrrr}9 & 8 & 15 & 23 & 37 & -42 \\ 10 & 8 & 15 & 71 & -15 & -19\end{array}$

$11 \quad 8 \quad 15 \quad 35 \quad 3 \quad-39$

$\begin{array}{lllllll}12 & 0 & 15 & 68 & 14 & -27 \\ -3 & 9 & 15 & 36 & -71 & -41\end{array}$

$\begin{array}{llllll}-2 & 9 & 15 & 109 & 111 & 15\end{array}$

$\begin{array}{llllll}-1 & 9 & 15 & 137 & -115 & 12\end{array}$

$1915 \quad 158-151 \quad 10$

$\begin{array}{llllll}2 & 9 & 15 & 120 & 114 & 12\end{array}$

$\begin{array}{llllll}3 & 9 & 15 & 131 & -1.43 & 11\end{array}$

$\begin{array}{rrrrrr}4 & 9 & 1.5 & 161 & 178 & 10 \\ 5 & 9 & 15 & 130 & -131 & 11\end{array}$

$6 \quad 9 \quad 15 \quad 154 \quad 143 \quad 10$

$\begin{array}{llllll}7 & 9 & 15 & 99 & -76 & 34\end{array}$

$\begin{array}{llllll}8 & 9 & 15 & 28 & 48 & -41\end{array}$

$\begin{array}{rrrrrr}9 & 9 & 15 & 47 & -54 & -27 \\ 10 & 9 & 15 & 53 & 25 & -26\end{array}$

$11 \quad 915 \quad 66 \quad 20-20$

$\begin{array}{rrrrrr}1.2 & 9 & 15 & 49 & 3 & -35 \\ -2 & 30 & 15 & 83 & -43 & -24\end{array}$

$\begin{array}{rrrrrr}-1 & 10 & 15 & 49 & 58 & -34 \\ 0 & 10 & 15 & 79 & -25 & -17\end{array}$

$\begin{array}{llllll}1 & 10 & 35 & 100 & -31 & 14\end{array}$

$\begin{array}{llllll}2 & 10 & 15 & 79 & -21 & -21\end{array}$

$\begin{array}{rrrrrr}3 & 10 & 15 & 45 & -19 & -31 \\ 4 & 10 & 15 & 35 & 44 & -35\end{array}$

$\begin{array}{lllllll}5 & 10 & 15 & 47 & -16 & -31\end{array}$

$\begin{array}{llllll}6 & 10 & 15 & 89 & 15 & 15\end{array}$

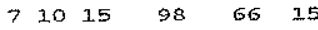

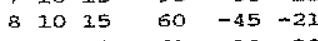

$\begin{array}{rrrrrr}9 & 10 & 15 & 63 & 39 & -20 \\ 10 & 10 & 15 & 79 & 3 & -38\end{array}$

$111015 \quad 51 \quad-4 \quad-35$

$\begin{array}{llllll}0 & 11 & 15 & 108 & 123 & 16\end{array}$

$\begin{array}{rrrrrr}1 & 11 & 15 & 97 & -112 & 15 \\ 2 & 11 & 15 & 90 & 97 & 17\end{array}$

$\begin{array}{llllll}3 & 11 & 15 & 1.47 & -142 & 10\end{array}$

$\begin{array}{llllll}4 & 13 & 15 & 131 & 123 & 12\end{array}$

$\begin{array}{llllll}5 & 11 & 1.5 & 104 & -90 & 15\end{array}$

$\begin{array}{llllll}6 & 11 & 15 & 201 & 127 & 16\end{array}$

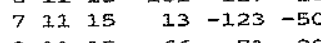

$\begin{array}{rrrrrr}8 & 11 & 15 & 66 & 71 & -20 \\ 9 & 11 & 15 & 33 & -19 & -33\end{array}$

$\begin{array}{llllll}10 & 11 & 15 & 59 & 7 & -23\end{array}$

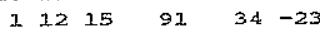

$\begin{array}{llllll}2 & 12 & 15 & 49 & -15 & -35 \\ 3 & 12 & 15 & 59 & -28 & -25\end{array}$

$\begin{array}{llllrr}4 & 12 & 15 & 47 & 53 & -27\end{array}$

$\begin{array}{rrrrr}512 & 15 & 93 & -70 & 15\end{array}$

$\begin{array}{rrrrrr}6 & 12 & 155 & 95 & 67 & 16 \\ 7 & 12 & 2.5 & 80 & -14 & -1.8\end{array}$

$\begin{array}{llllll}8 & 12 & 15 & 35 & 38 & -42\end{array}$

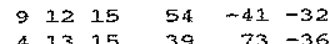

$\begin{array}{lllllll}5 & 13 & 15 & 35 & -79 & -41\end{array}$

$\begin{array}{llllll}6 & 13 & 15 & 52 & 45 & -35\end{array}$

$\begin{array}{llllll}7 & 13 & 15 & 14 & -77 & -55\end{array}$

$\begin{array}{rrrrrr}-2 & -8 & 16 & 68 & 42 & -19 \\ -1 & -8 & 16 & 45 & -34 & -27\end{array}$

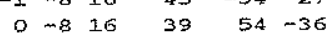

$\begin{array}{llllll}1 & -8 & 16 & 74 & -76 & -22\end{array}$

$\begin{array}{llllll}2 & -8 & 26 & 95 & 69 & 16\end{array}$

$\begin{array}{llllll}-4 & -7 & 16 & 56 & 20 & -31 \\ -3 & -7 & 16 & 39 & -1 & -36\end{array}$

$\begin{array}{llllll}-2 & -7 & 15 & 42 & -2 & -34\end{array}$

$\begin{array}{llllll}-1 & -7 & 1.6 & 29 & -18 & -41\end{array}$

$\begin{array}{lllll}-7 & 16 & 59 & 55 & -27\end{array}$

$\begin{array}{llllll}1 & -7 & 16 & 90 & -14 & -22\end{array}$

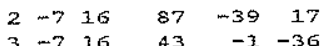

$\begin{array}{lllll}4-7 & 16 & 43 & -1 & -36 \\ 40 & -11 & -35\end{array}$

$\begin{array}{llllll}-5 & -6 & 16 & 87 & -58 & 16\end{array}$

$\begin{array}{llllll}-4 & -6 & 16 & 58 & 66 & -24\end{array}$

$\begin{array}{rrrrrr}-3 & -6 & 16 & 51 & -83 & -27 \\ -3 & -6 & 16 & 111 & 90 & 14\end{array}$

$\begin{array}{llllll}-1 & -6 & 16 & 102 & -77 & 14\end{array}$

$\begin{array}{llllll}0 & -6 & 16 & 43 & 88 & -35\end{array}$

$\begin{array}{rrrrrr}2 & -6 & 16 & 79 & -54 & -17 \\ 2 & -6 & 16 & 121 & 89 & 12\end{array}$

$\begin{array}{llllll}3 & -6 & 16 & 133 & -146 & 13\end{array}$

$\begin{array}{llllll}4 & -6 & 16 & 81 & 88 & -19\end{array}$ 


\begin{tabular}{|c|c|c|c|c|c|c|c|c|c|}
\hline 5 & -6 & 26 & 92 & -84 & 17 & 4 & 0 & 16 & 263 \\
\hline 6 & -6 & 16 & 96 & 86 & 17 & 5 & 0 & 16 & 174 \\
\hline-6 & -5 & 26 & 100 & 32 & 16 & 6 & 0 & 16 & 167 \\
\hline-5 & -5 & 1.5 & 30 & -27 & -41 & 7 & 0 & 16 & 76 \\
\hline-4 & -5 & 16 & 34 & -6 & -41 & 8 & 0 & 16 & 48 \\
\hline-3 & -5 & 16 & 46 & 3 & -32 & 9 & o & 16 & 57 \\
\hline-2 & -5 & 16 & 45 & -25 & -33 & 10 & 0 & 16 & 75 \\
\hline-1 & -5 & 16 & 22 & -5 & -42 & 21 & 0 & 16 & 80 \\
\hline 0 & -5 & 16 & 41 & 47 & -33 & -6 & 1 & 16 & 50 \\
\hline 1 & -5 & 16 & 88 & -21 & -19 & -5 & 1 & 16 & 33 \\
\hline 2 & -5 & 36 & 67 & 31 & -26 & -4 & 1 & 16 & 76 \\
\hline 3 & -5 & 16 & 93 & -19 & -20 & -3 & 1 & 16 & 44 \\
\hline 4 & -5 & 16 & 53 & $2 z$ & -27 & -2 & 1 & 16 & 45 \\
\hline 5 & -5 & 16 & 42 & -24 & -28 & -1 & 1 & 16 & 35 \\
\hline 6 & -5 & 16 & 31 & 3 & -44 & 0 & 1 & 16 & 24 \\
\hline 7 & -5 & 16 & 61 & 21 & -30 & 1 & 1 & 16 & 145 \\
\hline-6 & -4 & 16 & 75 & 55 & -22 & 2 & 1 & 26 & 43 \\
\hline-5 & -4 & 16 & 72 & -43 & -18 & 3 & 1 & 26 & 33 \\
\hline-4 & -4 & 16 & 108 & 78 & 13 & 4 & 1 & 16 & 53 \\
\hline-3 & -4 & 16 & 117 & -120 & 14 & 5 & 1 & 36 & 91 \\
\hline-2 & -4 & 16 & 117 & 116 & 13 & 6 & 1 & 16 & 33 \\
\hline-1 & -4 & 16 & 150 & -139 & 11 & 7 & 1 & 16 & 48 \\
\hline 0 & -4 & 16 & 109 & 118 & 13 & 8 & 1 & 15 & 37 \\
\hline 1 & -4 & 16 & 90 & -120 & 16 & 9 & 3 & 16 & 33 \\
\hline 2 & -4 & 16 & 56 & 93 & -22 & 10 & 1 & 16 & 89 \\
\hline 3 & -4 & 16 & 130 & -103 & 11 & 11 & 1 & 16 & 83 \\
\hline 4 & $-d_{2}$ & 16 & 233 & 218 & 12 & -6 & 2 & 16 & 184 \\
\hline 5 & -4 & 16 & 49 & -82 & -32 & -5 & 2 & 16 & 135 \\
\hline 6 & -4 & 16 & 134 & 125 & 12 & -4 & 2 & 16 & 115 \\
\hline 7 & -4 & 16 & 210 & -1.15 & 16 & -3 & 2 & 16 & 282 \\
\hline 8 & -4 & 16 & 49 & 81 & -38 & -2 & 2 & 26 & 177 \\
\hline-6 & -3 & 26 & 35 & 23 & -43 & -1 & 2 & 26 & 72 \\
\hline-5 & -3 & 26 & 13 & -45 & -53 & 0 & 2 & 16 & 230 \\
\hline-4 & -3 & 16 & 11 & 70 & -45 & 2 & 2 & 16 & 268 \\
\hline-3 & -3 & 16 & 44 & -26 & -32 & 2 & 2 & 16 & 353 \\
\hline$m 2$ & -3 & 16 & 46 & 33 & -30 & 3 & 2 & 26 & 24 \\
\hline-1 & -3 & 16 & 91 & -55 & -20 & 4 & 2 & 26 & 242 \\
\hline 0 & -3 & 26 & 51 & 74 & -29 & 5 & 2 & 16 & 134 \\
\hline 1 & -3 & 16 & 46 & -47 & -29 & 6 & 2 & 16 & 198 \\
\hline 2 & -3 & 16 & 65 & -12 & -24 & 7 & 2 & 26 & 194 \\
\hline 3 & -3 & 16 & 34 & -74 & -34 & 8 & 2 & 16 & 138 \\
\hline 4 & -3 & 16 & 53 & 77 & -27 & 9 & 2 & 26 & 74 \\
\hline 6 & -3 & 16 & 61 & 28 & -25 & 10 & 2 & 16 & 62 \\
\hline 7 & -3 & 26 & 60 & 4 & -23 & 11 & 2 & 16 & 86 \\
\hline 8 & -3 & 16 & 44 & -18 & -36 & 12 & 2 & 16 & 52 \\
\hline 9 & -3 & 16 & 54 & 25 & -28 & -6 & 3 & 26 & 44 \\
\hline-6 & -2 & 16 & 75 & 61 & -27 & -5 & 3 & 26 & 24 \\
\hline-5 & -2 & 16 & $10 \%$ & -90 & 25 & -4 & 3 & 26 & 65 \\
\hline-4 & -2 & 16 & 48 & 69 & -32 & -3 & 3 & 16 & 78 \\
\hline-3 & -2 & 16 & 41 & -73 & -34 & -2 & 3 & 16 & 26 \\
\hline-2 & -2 & 16 & 152 & 155 & 10 & -1 & 3 & 16 & 39 \\
\hline-1 & -2 & 16 & 280 & -195 & 7 & 0 & 3 & 16 & 42 \\
\hline 0 & -2 & 16 & 225 & 232 & 8 & 1 & 3 & 16 & 77 \\
\hline 主 & -2 & 16 & 207 & -213 & 8 & 2 & 3 & 26 & 51 \\
\hline 2 & -2 & 16 & 226 & 205 & 7 & 3 & 3 & 16 & 102 \\
\hline 3 & -2 & 16 & 211 & -190 & 8 & 4 & 3 & 16 & 28 \\
\hline 4 & -2 & 16 & 86 & 82 & 16 & 5 & 3 & 16 & 56 \\
\hline 5 & $-z$ & 16 & 147 & -113 & 9 & 6 & 3 & 16 & 36 \\
\hline 6 & -2 & 26 & 166 & 148 & 10 & 7 & 3 & 16 & 37 \\
\hline 7 & -2 & 16 & 267 & -153 & 12 & 8 & 3 & 36 & 96 \\
\hline 8 & -2 & 16 & 66 & 82 & -29 & 9 & 3 & 26 & 52 \\
\hline 9 & -2 & 16 & 65 & -61 & -30 & 10 & 3 & 16 & 29 \\
\hline 10 & -2 & 16 & 49 & 48 & -35 & 11 & 3 & 16 & 43 \\
\hline-5 & -1 & 16 & 61 & 22 & -28 & 12 & 3 & 16 & 순 2 \\
\hline-5 & -2 & 16 & 60 & 12 & -28 & -5 & 4 & 16 & 55 \\
\hline$-\frac{\lambda}{x}$ & -1 & 16 & 78 & -7 & -19 & -4 & 4 & 16 & 117 \\
\hline-3 & -1 & 16 & 68 & -57 & -17 & -3 & 4 & 16 & 104 \\
\hline-2 & -1 & 16 & 59 & 38 & -23 & -2 & d & 16 & 175 \\
\hline-1 & -1 & 16 & 54 & -5 & -26 & -1 & 4 & 16 & 176 \\
\hline 0 & -1 & 16 & 152 & 64 & 23 & 0 & 4 & 16 & 185 \\
\hline 1 & -1 & 16 & 90 & -133 & 14 & 1 & 4 & 16 & 191 \\
\hline 2 & -1 & 26 & 131 & 149 & 12 & 2 & 4 & 16 & 243 \\
\hline 3 & -1 & 16 & 59 & -60 & -25 & 3 & 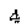 & 16 & 195 \\
\hline 4 & -1 & 16 & 69 & 58 & -18 & 4 & 4 & 26 & 166 \\
\hline 5 & -1 & 16 & 70 & -17 & -23 & 5 & 4 & 16 & 193 \\
\hline 6 & -1 & 16 & 59 & 6 & -18 & E & 4 & 16 & 325 \\
\hline 7 & -1 & 16 & 39 & -6 & -31 & 7 & 4 & 26 & 147 \\
\hline 8 & -1 & 16 & 12 & 19 & -49 & 8 & 4 & 16 & 166 \\
\hline 9 & -1 & 16 & 74 & -7 & -28 & 9 & \pm & 16 & 111 \\
\hline 10 & -1 & 16 & 61 & -25 & -33 & 10 & $\stackrel{d}{=}$ & 16 & 56 \\
\hline-6 & o & 16 & 74 & 69 & -17 & 11 & 4 & 16 & 92 \\
\hline-5 & 0 & 16 & 91 & -79 & 12 & 12 & 4 & 16 & 63 \\
\hline-4 & 0 & 16 & 137 & 128 & 8 & -4 & 5 & 16 & 46 \\
\hline-3 & 0 & 16 & 116 & -88 & 19 & -3 & 5 & 16 & 52 \\
\hline-2 & 0 & 16 & 73 & 17 & 2.3 & -2 & 5 & 16 & 65 \\
\hline-1 & 0 & 16 & 157 & -129 & 22 & -1 & 5 & 26 & 46 \\
\hline 0 & 0 & 16 & 173 & 170 & 9 & 0 & 5 & 16 & 75 \\
\hline 3 & o & 15 & 182 & -165 & 9 & 1 & 5 & 16 & 82 \\
\hline 2 & o & 16 & 123 & 119 & 7 & 2 & 5 & 16 & 115 \\
\hline
\end{tabular}

$\begin{array}{rrr}5 & 5 & 16 \\ 6 & 5 & 16 \\ 7 & 5 & 16 \\ 8 & 5 & 16 \\ 9 & 5 & 16 \\ 10 & 5 & 16 \\ 11 & 5 & 16 \\ 12 & 5 & 16 \\ -4 & 6 & 16 \\ -3 & 6 & 16 \\ -2 & 6 & 16 \\ -1 & 6 & 16 \\ 0 & 6 & 16 \\ 1 & 6 & 16 \\ 2 & 6 & 16 \\ 3 & 6 & 16 \\ 4 & 6 & 16 \\ 5 & 6 & 16 \\ 6 & 6 & 16 \\ 7 & 6 & 16 \\ 8 & 6 & 16 \\ 9 & 6 & 16 \\ 10 & 6 & 36 \\ 13 & 6 & 16 \\ 12 & 6 & 16 \\ -4 & 7 & 16 \\ -3 & 7 & 16 \\ -2 & 7 & 16 \\ -1 & 7 & 16 \\ 1 & 7 & 16 \\ 2 & 7 & 16 \\ 3 & 7 & 16 \\ 4 & 7 & 16 \\ 5 & 7 & 16 \\ 6 & 7 & 16 \\ 7 & 7 & 16 \\ 8 & 7 & 16 \\ 9 & 7 & 16 \\ 10 & 7 & 16 \\ 11 & 7 & 16\end{array}$

11716

$\begin{array}{llllll}12 & 7 & 1.6 & 71 & 21 & -41 \\ -3 & 8 & -23\end{array}$

$\begin{array}{llllll}-3 & 8 & 16 & 112 & -105 & 15\end{array}$

$\begin{array}{llllll}-2 & 8 & 16 & 132 & 216 & 12\end{array}$

$\begin{array}{llllll}- & 8 & 16 & 111 & 118 & 12\end{array}$

$1816 \quad 108-135 \quad 14$

$\begin{array}{llllll}2 & 8 & 16 & 130 & 129 & 11\end{array}$

$\begin{array}{rrrrrr}3 & 8 & 16 & 195 & -184 & 8 \\ 4 & 8 & 16 & 194 & 207 & 9\end{array}$

$\begin{array}{llllll}5 & 8 & 16 & 135 & -162 & 11\end{array}$

$\begin{array}{llllll}6 & 8 & 16 & 169 & 196 & 10\end{array}$

$\begin{array}{llllll}7 & 8 & 16 & 87 & -119 & 16\end{array}$

$\begin{array}{rrrrrr}8 & 8 & 16 & 72 & 64 & -18 \\ 9 & 8 & 16 & 85 & -64 & 17\end{array}$

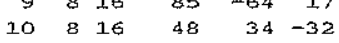

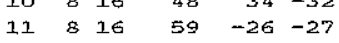

12816

$\begin{array}{lll}-2 & 9 & 16 \\ -1 & 9 & 16\end{array}$

- 926

1926

$\begin{array}{lll}2 & 9 & 26 \\ 3 & 9 & 26\end{array}$

$\begin{array}{lll}3 & 9 & 26 \\ 4 & 9 & 16 \\ 5 & 9 & 16\end{array}$

6916

7916

$\begin{array}{lll}8 & 9 & 16 \\ 9 & 9 & 16\end{array}$

10916

$\begin{array}{lll}1.1 & 10 & 16\end{array}$

01016

$\begin{array}{lll}1 & 10 & 16 \\ 2 & 10 & 16\end{array}$

$\begin{array}{rrrrrr}3 & 10 & 16 & 88 & -97 & 15\end{array}$

$\begin{array}{llllll}5 & 10 & 16 & 125 & -132 & 12\end{array}$

$\begin{array}{llllll}6 & 10 & 16 & 154 & 3.47 & 11\end{array}$

$\begin{array}{llllll}7 & 10 & 16 & 107 & -81 & 13\end{array}$

$\begin{array}{llllll}8 & 10 & 16 & 12 & 31 & -48 \\ 9 & 10 & 16 & 51 & -7 & -30\end{array}$

$\begin{array}{rlllll}9 & 10 & 16 & 51 & -7 & -30 \\ 10 & 10 & 16 & 33 & -1 & -36\end{array}$

111016

11116

$\begin{array}{lll}2 & 11 & 16 \\ 3 & 11 & 15\end{array}$

41210

90
79

$32-34$

$\begin{array}{ll}-2 & -21 \\ 15 & -24\end{array}$

$4-28$

$\begin{array}{lll}4 & 11 & 16 \\ 5 & 11 & 16\end{array}$
$61116 \quad 12 \quad 25-49$

$81116 \quad 75 \quad 53-19$

$9 \begin{array}{lllll}911 & 16 & 73 & -35 & -20\end{array}$

$101116 \quad 44 \quad 4 \quad-37$

$\begin{array}{llllll}2 & 12 & 16 & 45 & 78 & -28\end{array}$

$\begin{array}{rrrrrr}3 & 12 & 16 & 42 & -59 & -32 \\ 4 & 12 & 16 & 116 & 119 & 15\end{array}$

$\begin{array}{llllll}5 & 12 & 16 & 59 & -85 & -29\end{array}$

$\begin{array}{llllll}6 & 12 & 16 & 71 & 98 & -23\end{array}$

$\begin{array}{llllll}7 & 12 & 16 & 77 & -70 & -20\end{array}$

$\begin{array}{rrrrrr}8 & 12 & 16 & 58 & 23 & -28 \\ 9 & 12 & 16 & 76 & -55 & -20\end{array}$

$\begin{array}{llllll}9 & 12 & 16 & 76 & 55 & -20 \\ -2 & -7 & 17 & 58 & 35 & -24\end{array}$

$\begin{array}{llllll}-1 & -7 & 17 & 74 & -45 & -25\end{array}$

$\begin{array}{llllll}0 & -7 & 17 & 93 & 71 & 15\end{array}$

$\begin{array}{llllll}1 & -7 & 17 & 93 & -61 & -23\end{array}$

$\begin{array}{rrrrrr}2 & -7 & 17 & 12 & 71 & -49 \\ 3 & -7 & 17 & 13 & -39 & -53\end{array}$

$\begin{array}{rrrrrr}3 & -7 & 17 & 13 & -39 & -53 \\ -4 & -6 & 17 & 36 & 25 & -40\end{array}$

$-3-6,7$

$\begin{array}{lll}-3 & -6 & 1.7 \\ -2 & -6 & 1.7\end{array}$

$\begin{array}{llll}-1 & -6 & 17\end{array}$

$1-617$

$\begin{array}{llll}2 & -6 & 37\end{array}$

$\begin{array}{lll}3 & -6 & 17\end{array}$

$\begin{array}{lll}4 & -6 & 3.7 \\ 5 & -6 & 37\end{array}$

$\begin{array}{lll}-5 & -5 & 17\end{array}$

$\begin{array}{lll}-4 & -5 & 17\end{array}$

$\begin{array}{lll}-3 & -5 & 17 \\ -2 & -5 & 17\end{array}$

$\begin{array}{lll}-2 & -5 & 17 \\ -1 & -5 & 17\end{array}$

- $-5 \quad 17$

$\begin{array}{llll}1 & -5 & 17\end{array}$

$\begin{array}{lll}2 & -5 & 17\end{array}$

$\begin{array}{lll}3 & -5 & 17 \\ 4 & -5 & 17\end{array}$

$\begin{array}{lll}5 & -5 & 17\end{array}$

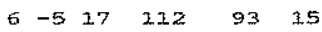

$\begin{array}{rrr}39 & -37 & -35 \\ 51 & 9 & -32\end{array}$

$\begin{array}{rrr}51 & 9 & -32 \\ 26 & -4 & -32\end{array}$

$\begin{array}{lll}34 & 13 & -38\end{array}$

$65-29-25$

$29 \quad 28-40$

$\begin{array}{rrr}80 & -7 & -16 \\ 91 & 5 & 15\end{array}$

$\begin{array}{lll}63 & 20 & -29\end{array}$

$65-21-29$

$\begin{array}{rrr}62 & 66 & 14 \\ 62 & -89 & -23\end{array}$

$99 \quad 83 \quad 15$

$87-70 \quad 15$

$97-101 \quad 25$

$\begin{array}{rrr}97 & -101 & 15 \\ 99 & 64 & 16\end{array}$

$91 \quad-78 \quad 36$

$76 \quad 81-20$

$\begin{array}{llllll}-5 & -4 & 17 & 73 & -30 & -25\end{array}$

$\begin{array}{rrrrrr}-4 & -4 & 17 & 57 & 34 & -28 \\ -3 & -4 & 17 & 23 & -16 & -46\end{array}$

$\begin{array}{lll}-2 & -4 & 17\end{array}$

$\begin{array}{lll}-1 & -4 & 17\end{array}$

$\begin{array}{lll}0 & -4 & 17\end{array}$

$\begin{array}{lll}1 & -4 & 17 \\ 2 & -4 & 17\end{array}$

$\begin{array}{llll}3 & -4 & 17\end{array}$

$\begin{array}{lll}4 & -4 \quad 17\end{array}$

$5-4 \quad 17$

6. $-4 \quad 17$

$\begin{array}{rrr}7 & -4 & 17 \\ -5 & -3 & 17\end{array}$

$\begin{array}{lll}-3 & -3 & 17\end{array}$

$\begin{array}{lll}-2 & -3 & 27\end{array}$

$\begin{array}{lll}-2 & -3 & 17 \\ -1 & -3 & 17\end{array}$

$\begin{array}{lll}0 & -3 & 17 \\ 1 & -3 & 17\end{array}$

$2-3$ in

$\begin{array}{lll}3 & -3 & 17\end{array}$

$\begin{array}{lll}4 & -3 & 27 \\ 5 & -3 & 37\end{array}$

$\begin{array}{lll}6 & -3 & 1 \\ 7 & -3 & 17\end{array}$

$\begin{array}{lll}7 & -3 & 17\end{array}$

$\begin{array}{rrr}8 & -3 & 17 \\ -6 & -2 & 17\end{array}$

$\begin{array}{llll}-4 & -2 & 17\end{array}$

$\begin{array}{lll}-3 & -2 & 17 \\ -2 & -2 & 17\end{array}$

$\begin{array}{lll}-2 & -2 & 17 \\ -1 & -2 & 17\end{array}$

$\begin{array}{lll}0 & -2 & 17 \\ 1 & -2 & 17 \\ 2 & -2 & 17\end{array}$

$\begin{array}{lll}2 & -2 & 17 \\ 3 & -2 & 17\end{array}$

$\begin{array}{lll}4 & -2 & 17\end{array}$

$5-217$

$\begin{array}{lll}6 & -2 & 17\end{array}$

$\begin{array}{lll}7 & -2 & 17 \\ 8 & -2 & 17\end{array}$

$\begin{array}{lll}8 & -2 & 17 \\ 9 & -2 & 17\end{array}$

$\begin{array}{lll}-6 & -1 & 17\end{array}$

$\begin{array}{lll}-5 & -1 & 17 \\ -4 & -1 & 17\end{array}$

$\begin{array}{lll}-4 & -1 & 17 \\ -3 & -1 & 17\end{array}$

$\begin{array}{lll}-2 & -1 & 17 \\ -1 & -1 & 17\end{array}$

$\begin{array}{lll}0 & -1 & 17 \\ 1 & -1 & 17\end{array}$ $\begin{array}{llllll}2 & -1 & 27 & 344 & 134 & 10\end{array}$ $\begin{array}{llllll}3 & -1 & 17 & 244 & -227 & 8\end{array}$ $\begin{array}{rrrrrr}4 & -1 & 17 & 226 & 227 & 8 \\ 5 & -1 & 17 & 203 & -187 & 8\end{array}$ $\begin{array}{llllll}6 & -1 & 17 & 148 & 142 & 30\end{array}$ $\begin{array}{llllll}7 & -1 & 17 & 94 & -95 & 16\end{array}$ $\begin{array}{rrrrrr}8 & -1 & 17 & 122 & 89 & 23 \\ 10 & -1 & 17 & 89 & 81 & -19\end{array}$ $\begin{array}{llllll}-6 & 0 & 17 & 40 & -28\end{array}$ $\begin{array}{llllll}-5 & 0 & 17 & 87 & -12 & -36\end{array}$ $\begin{array}{llllll}-4 & 0 & 17 & 74 & -48 & -19\end{array}$ $\begin{array}{llllll}-3 & 0 & 17 & 32 & 22 & -26\end{array}$ $\begin{array}{llllll}-2 & 0 & 17 & 40 & 31 & -22 \\ -1 & 0 & 17 & 77 & -4 & -15\end{array}$

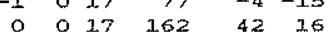
$\begin{array}{llllll}1 & 0 & 17 & 122 & -61 & 16\end{array}$ $\begin{array}{rrrrrr}2 & 0 & 17 & 55 & 40 & -15 \\ 3 & 0 & 17 & 54 & 44 & -17\end{array}$ $4017 \quad 11 \quad 7-32$ $\begin{array}{llllll}5 & 0 & 17 & 48 & -51 & -21\end{array}$ $\begin{array}{llllll}6 & 0 & 17 & 36 & 52 & -23\end{array}$ $\begin{array}{llllll}7 & 0 & 17 & 93 & -19 & -22\end{array}$ $\begin{array}{rrrrrr}8 & 0 & 17 & 55 & -20 & -18 \\ 9 & 0 & 17 & 78 & 13 & -18\end{array}$

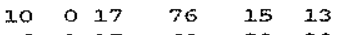
$\begin{array}{llllll}-6 & 1 & 17 & 50 & 50 & -30\end{array}$ $\begin{array}{llllll}-5 & 1 & 17 & 100 & -70 & 15\end{array}$ $\begin{array}{rrrrrr}-4 & 1 & 17 & 58 & 84 & -31 \\ -3 & 1 & 17 & 138 & -145 & 12\end{array}$ $\begin{array}{llllll}-2 & 1 & 17 & 109 & 105 & 12\end{array}$ $\begin{array}{llllll}-1 & 1 & 17 & 56 & -70 & -25\end{array}$ - $127 \quad 174 \quad 175$ $\begin{array}{rrrrrr}3 & 1 & 17 & 172 & -166 & 9 \\ 2 & 1 & 17 & 108 & 97 & 11\end{array}$ $\begin{array}{llllll}3 & 1 & 17 & 153 & -147 & 8\end{array}$ $\begin{array}{llllll}4 & 3 & 37 & 205 & 203 & 8\end{array}$ $\begin{array}{llllll}5 & 3 & 27 & 169 & -174 & 10\end{array}$ $\begin{array}{llllll}6 & 1 & 17 & 387 & 188 & 10\end{array}$ $\begin{array}{rrrrrr}7 & 1 & 17 & 158 & -159 & 11 \\ 8 & 1 & 17 & 98 & 103 & 15\end{array}$ $\begin{array}{llllll}9 & 1 & 17 & 126 & -98 & 13\end{array}$ $\begin{array}{llllll}10 & 3 & 27 & 87 & 27 & -23\end{array}$ $\begin{array}{llllll}11 & 1 & 37 & 74 & -18 & -22\end{array}$

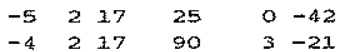
$\begin{array}{llllll}-3 & 2 & 17 & 43 & 13 & -32\end{array}$ $\begin{array}{llllllll}-2 & 2 & 17 & 75 & -18 & -22\end{array}$ $\begin{array}{llllll}-1 & 2 & 17 & 45 & 49 & -27\end{array}$ $\begin{array}{rrrrrr}0 & 2 & 17 & 95 & -6 & 18 \\ 1 & 2 & 17 & 118 & 107 & 19\end{array}$ $\begin{array}{llllll}2 & 2 & 17 & 103 & -71 & 17\end{array}$ $\begin{array}{llllll}4 & 2 & 17 & 25 & -63 & -36\end{array}$ $5 \quad 217 \quad 103 \quad 101 \quad 13$ $\begin{array}{rrrrrr}6 & 2 & 17 & 52 & -27 & -27 \\ 7 & 2 & 17 & 95 & 64 & 15\end{array}$

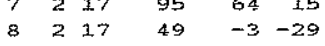
$\begin{array}{lllll}9 & 27 & 41 & -18 & -34\end{array}$ $\begin{array}{llllll}10 & 2 & 17 & 70 & -5 & -28 \\ 11 & 2 & 17 & 66 & 11 & -28\end{array}$ $\begin{array}{rrrrrr}11 & 2 & 17 & 66 & 11 & -28 \\ -5 & 3 & 17 & 110 & -73 & 14\end{array}$ $\begin{array}{llllll}-4 & 3 & 17 & 76 & 61 & -18\end{array}$ $\begin{array}{rrrrrr}-3 & 3 & 17 & 64 & -106 & -29 \\ -2 & 3 & 17 & 193 & 175 & 9\end{array}$ $\begin{array}{llllll}-1 & 3 & 17 & 153 & -160 & 10\end{array}$ $\begin{array}{rrrrrr}0 & 3 & 17 & 229 & 230 & 8 \\ 1 & 3 & 17 & 128 & -139 & 11\end{array}$ $\begin{array}{rrrrrr}1 & 3 & 17 & 128 & -139 & 11 \\ 2 & 3 & 17 & 118 & 100 & 11\end{array}$ $\begin{array}{lllllr}3 & 3 & 17 & 156 & -148 & 9\end{array}$ $\begin{array}{llllll}4 & 3 & 17 & 200 & 173 & 8\end{array}$ $5 \quad 317 \quad 177-191 \quad 10$ $\begin{array}{llllll}6 & 3 & 17 & 117 & 138 & 14 \\ 7 & 3 & 17 & 77 & 046 & -19\end{array}$ $\begin{array}{llllll}8 & 3 & 17 & 32 & 45 & -39\end{array}$ $\begin{array}{llllll}9 & 3 & 17 & 60 & -54 & -27\end{array}$ $\begin{array}{llllll}10 & 3 & 17 & 101 & 46 & 25\end{array}$ $\begin{array}{rrrrrr}12 & 3 & 37 & 76 & -24 & -19 \\ -5 & 4 & 17 & 71 & 29 & -19\end{array}$ $\begin{array}{llllll}-4 & 4 & 17 & 52 & -31 & -29\end{array}$ $\begin{array}{llllll}-3 & 4 & 17 & 33 & -1 & -35\end{array}$ $\begin{array}{llllll}-2 & 4 & 17 & 39 & -7 & -33 \\ -1 & 4 & 17 & 27 & -9 & -38\end{array}$ - $417 \quad 89 \quad 86 \quad 15$ $\begin{array}{rrrrrr}1 & 4 & 17 & 92 & 42 & -20 \\ 2 & 4 & 17 & 102 & -59 & 37\end{array}$ $\begin{array}{rrrrrr}2 & 4 & 17 & 102 & -59 & 17 \\ 3 & 4 & 17 & 25 & 117 & -44\end{array}$ $\begin{array}{rrrrrr}4 & 4 & 17 & 93 & -98 & 14 \\ 5 & 4 & 17 & 55 & 17 & -25\end{array}$ $6417 \quad 30 \quad-8-38$ $\begin{array}{rrrrrr}7 & 4 & 17 & 22 & -1 & -42 \\ 8 & 4 & 27 & 50 & -40 & -27\end{array}$ 


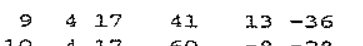

$\begin{array}{rrrrrr}10 & 4 & 17 & 60 & -8 & -28 \\ 11 & 4 & 17 & 65 & 9 & -28\end{array}$

$\begin{array}{rrrrrr}12 & 4 & 17 & 97 & 4 & 16 \\ -4 & 5 & 17 & 138 & 97 & 12\end{array}$

$\begin{array}{llllll}-3 & 5 & 27 & 105 & -85 & 14\end{array}$

$\begin{array}{rrrrrr}-2 & 5 & 17 & 101 & 90 & 14 \\ -1 & 5 & 17 & 124 & -124 & 17\end{array}$

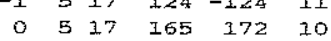

$\begin{array}{llllll}1 & 5 & 17 & 224 & -233 & 8\end{array}$

$\begin{array}{lllll}2 & 5 & 17 & 219 & 227\end{array}$

$\begin{array}{rrrrrr}3 & 5 & 17 & 175 & -173 & 9 \\ 4 & 5 & 17 & 190 & 191 & 9\end{array}$

$\begin{array}{llllll}5 & 5 & 17 & 62 & -107 & -29\end{array}$

$\begin{array}{llllll}6 & 5 & 17 & 144 & 138 & 11\end{array}$

$\begin{array}{rrrrrr}7 & 5 & 17 & 121 & -154 & 14 \\ 8 & 5 & 17 & 141 & 126 & 11\end{array}$

$9517 \quad 106-101 \quad 15$

$\begin{array}{llllll}10 & 5 & 37 & 89 & 47 & 15\end{array}$

$\begin{array}{llllll}11 & 5 & 17 & 56 & 1 & -30 \\ 12 & 5 & 17 & 59 & 9 & -32\end{array}$

$\begin{array}{rrrrrr}-4 & 6 & 17 & 66 & -18 & -21 \\ -3 & 6 & 17 & 71 & 56 & -28\end{array}$

$\begin{array}{llllll}-2 & 6 & 17 & 85 & -13 & -20\end{array}$

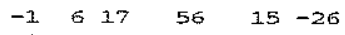

$\begin{array}{llllll}0 & 6 & 17 & 41 & 40 & -30 \\ 1 & 6 & 17 & 43 & -10 & -31\end{array}$

$\begin{array}{llllll}2 & 6 & 17 & 47 & -62 & -31 \\ 3 & 5 & 17 & 17 & -28 & -45\end{array}$

$\begin{array}{llllll}4 & 6 & 17 & 23 & 12 & -34\end{array}$

$\begin{array}{rrrrrr}5 & 6 & 17 & 21 & 26 & -42 \\ 6 & 6 & 17 & 59 & -47 & -25\end{array}$

7617 50 $48-25$

$9617 \quad 10 \quad-76-42$

$\begin{array}{llllll}10 & 6 & 17 & 34 & 68 & -41 \\ 12 & 6 & 17 & 86 & 30 & -18\end{array}$

$\begin{array}{rrrrrr}-3 & 7 & 17 & 40 & -101 & -39 \\ -2 & 7 & 17 & 173 & 139 & 10\end{array}$

$\begin{array}{llllll}-1 & 7 & 17 & 129 & -109 & 12\end{array}$

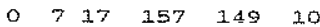

$\begin{array}{lllll}1 & 717 & 106 & -110 & 13\end{array}$

$\begin{array}{rrrrr}2 & 7 & 17 & 162 & 165 \\ 3 & 7 & 17 & 185 & -170\end{array}$

$\begin{array}{llllll}4 & 7 & 17 & 192 & 192\end{array}$

$\begin{array}{lllll}5 & 7 & 17 & 195 & -173\end{array}$

$\begin{array}{llllll}6 & 7 & 17 & 173 & 179 & 9\end{array}$

$\begin{array}{rrrrrr}7 & 7 & 17 & 104 & -74 & 13 \\ 8 & 7 & 17 & 85 & 47 & 16\end{array}$

$\begin{array}{llllll}9 & 7 & 17 & 29 & -17 & -42\end{array}$

$\begin{array}{llllll}10 & 7 & 17 & 57 & 31 & -28\end{array}$

$\begin{array}{llllll}11 & 7 & 17 & 48 & -55 & -32\end{array}$

$\begin{array}{llllll}-2 & 8 & 17 & 59 & -45 & -28\end{array}$

$\begin{array}{rrrrrr}-1 & 8 & 17 & 77 & 26 & -17 \\ 0 & 8 & 17 & 50 & 38 & -31\end{array}$

$\begin{array}{llllll}1 & 8 & 17 & 23 & -36 & -38\end{array}$

$\begin{array}{rrrrrr}2 & 8 & 17 & 55 & 25 & -27 \\ 3 & 8 & 17 & 51 & 2 & -21\end{array}$

$\begin{array}{rrrrrr}3 & 8 & 17 & 51 & 2 & -21 \\ 4 & 8 & 17 & 60 & 40 & -26 \\ 5 & 8 & 17 & 11 & -56 & -75\end{array}$

$\begin{array}{rrrrr}6 & 817 & 60 & 74 & -21\end{array}$

$\begin{array}{rrrrrr}7 & 8 & 17 & 70 & -82 & -19 \\ 8 & 8 & 17 & 29 & 33 & -41\end{array}$

$\begin{array}{llllrr}8 & 8 & 17 & 29 & 33 & -41 \\ 9 & 8 & 17 & 42 & -15 & -35\end{array}$

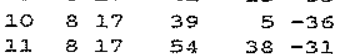

$\begin{array}{lllll}-1.917 & 90 & -84 & -18\end{array}$

$0 \quad 917 \quad 214 \quad 109 \quad 13$

$\begin{array}{llllll}1 & 9 & 17 & 119 & -110 & 13\end{array}$

$\begin{array}{rrrrrr}2 & 9 & 17 & 100 & 105 & 15 \\ 3 & 9 & 17 & 90 & -90 & 14\end{array}$

$\begin{array}{llllll}4 & 9 & 17 & 115 & 111 & 12\end{array}$

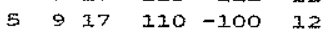

$\begin{array}{llllll}6 & 9 & 17 & 131 & 133 & 13\end{array}$

$\begin{array}{llllll}7 & 9 & 17 & 117 & -132 & 14 \\ 8 & 9 & 17 & 114 & 109 & 15\end{array}$

$9 \quad 917 \quad 101-102 \quad 18$

$\begin{array}{llllll}10 & 9 & 17 & 69 & 46 & -22\end{array}$

$\begin{array}{rrrrrr}11 & 9 & 17 & 37 & -42 & -42 \\ 0 & 10 & 17 & 60 & -5 & -22\end{array}$

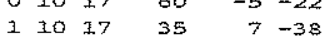

$21017 \quad 58 \quad 10-22$

$\begin{array}{llllll}3 & 10 & 17 & 33 & -25 & -37\end{array}$

$\begin{array}{llllll}5 & 10 & 17 & 57 & -2 & -27\end{array}$

$\begin{array}{llllll}6 & 30 & 17 & 62 & -19 & -27\end{array}$

$\begin{array}{llllll}7 & 10 & 17 & 37 & -25 & -36\end{array}$

$81017 \quad 10 \quad 15-41$

$\begin{array}{llllll}9 & 10 & 17 & 35 & -33 & -41\end{array}$

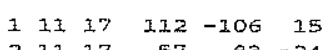

$\begin{array}{lllllll}2 & 11 & 17 & 57 & 83 & -24 \\ 3 & 13 & 17 & 82 & -66 & -28\end{array}$

$\begin{array}{rrrrrr}3 & 11 & 17 & 82 & -66 & -28 \\ 4 & 111 & 17 & 53 & 75 & -25\end{array}$

$\begin{array}{llllll}5 & 11 & 17 & 88 & -65 & 3.6\end{array}$

$\begin{array}{rrrrrr}6 & 11 & 17 & 121 & 101 & 12 \\ 7 & 11 & 17 & 75 & -97 & -19\end{array}$

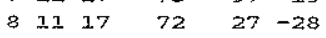

$\begin{array}{llllll}9 & 11 & 17 & 88 & -48 & -18\end{array}$

$\begin{array}{llllll}4 & 12 & 17 & 10 & 8 & -39\end{array}$

$\begin{array}{rrrrrr}5 & 12 & 17 & 63 & -13 & -25 \\ 7 & 12 & 17 & 87 & -10 & 16\end{array}$

$\begin{array}{llllll}-2 & -6 & 18 & 61 & 49 & -31\end{array}$

$\begin{array}{llllll}-1 & -6 & 18 & 43 & -36 & -37\end{array}$

$\begin{array}{rrrrrr}0 & -6 & 18 & 42 & 61 & -36 \\ 1 & -6 & 18 & 65 & -62 & -21\end{array}$

$\begin{array}{rrrrrr}2 & -6 & 18 & 50 & 49 & -32 \\ 3 & -6 & 18 & 53 & -57 & -30\end{array}$

$\begin{array}{llllll}4 & -6 & 18 & 61 & 45 & -22\end{array}$

$\begin{array}{llllll}-3 & -5 & 18 & 50 & -21 & -33\end{array}$

$\begin{array}{rrrrrr}-2 & -5 & 18 & 91 & 22 & 15 \\ -1 & -5 & 18 & 54 & -33 & -28\end{array}$

$\begin{array}{lllll}-5 & 18 & 81 & 46 & -22\end{array}$

$\begin{array}{llllll}-5 & 18 & 84 & -10 & -23\end{array}$

$\begin{array}{rrrrrr}2 & -5 & 18 & 51 & 8 & -27 \\ 4 & -5 & 18 & 72 & -14 & -25\end{array}$

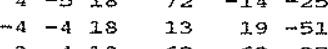

$\begin{array}{llllll}-3 & -4 & 18 & 62 & -62 & -27\end{array}$

$\begin{array}{rrrrrr}-1 & -4 & 18 & 57 & -45 & -27\end{array}$

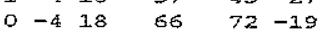

$\begin{array}{rrrrrr}1 & -4 & 18 & 87 & -100 & 16 \\ 2 & -4 & 18 & 119 & 104 & 13\end{array}$

$\begin{array}{lllll}3-4 & 18 & 128 & -81 & 11\end{array}$

$\begin{array}{llllll}4 & -4 & 18 & 82 & 53 & -17\end{array}$

$\begin{array}{llllll}5 & -4 & 18 & 80 & -70 & -20 \\ 5 & -4 & 18 & 69 & 102 & -29\end{array}$

$\begin{array}{llllll}7 & -4 & 18 & 84 & -67 & -19 \\ -4 & -3 & 18 & 43 & 23 & -34\end{array}$

$\begin{array}{llllll}-3 & -3 & 18 & 24 & -27 & -41\end{array}$

$\begin{array}{lll}-2 & -3 & 18\end{array}$

$\begin{array}{lll}-1 & -3 & 18 \\ 0 & -3 & 18\end{array}$

$\begin{array}{lll}0 & -3 & 28 \\ 1 & -3 & 18\end{array}$

$2-318$

$\begin{array}{lll}3 & -3 & 18\end{array}$

$\begin{array}{lll}4 & -3 & 18 \\ 5 & -3 & 18\end{array}$

$6-3 \quad 18$

$\begin{array}{lll}7 & -3 & 18\end{array}$

$\begin{array}{lll}8 & -3 & 18\end{array}$

$-5-218$

$\begin{array}{rrr}24 & -27 & -41 \\ 59 & 0 & -28\end{array}$

$\begin{array}{rrr}74 & -24 & -23 \\ 55 & 65 & -26\end{array}$

$\begin{array}{lll}39 & -26 & -33\end{array}$

$12-30-46$

$\begin{array}{lll}75 & 15 & -24\end{array}$

$\begin{array}{rrr}23 & -16 & -43 \\ 29 & 33 & -34\end{array}$

$69-18-25$

$\begin{array}{rrrrrr}-4 & -2 & 18 & 75 & 60 & -19 \\ -3 & -2 & 18 & 29 & -57 & -41\end{array}$

$\begin{array}{rrrrrr}-2 & -2 & 18 & 53 & 67 & -24\end{array}$

$\begin{array}{llllll}1 & -2 & 18 & 105 & -86 & 14 \\ 0 & -2 & 18 & 102 & 108 & 14\end{array}$

$\begin{array}{rrrrrr}0 & -2 & 18 & 302 & 108 & 14 \\ 1 & -2 & 18 & 202 & -107 & 14\end{array}$

$\begin{array}{rrrrrr}2 & -2 & 18 & 154 & 142 & 10 \\ 3 & -2 & 18 & 160 & -134 & 10\end{array}$

$\begin{array}{rrrrrr}3 & -2 & 18 & 160 & -134 & 10 \\ 4 & -2 & 18 & 73 & 81 & -18\end{array}$

$\begin{array}{llllll}5 & -2 & 18 & 103 & -82 & 14\end{array}$

$\begin{array}{llllll}6 & -2 & 18 & 115 & 112 & 14\end{array}$

$\begin{array}{rrrrrr}7 & -2 & 18 & 86 & -113 & -19 \\ 8 & -2 & 18 & 68 & 53 & -22\end{array}$

$\begin{array}{rrrrrr}8 & -2 & 18 & 68 & 51 & -22 \\ 9 & -2 & 18 & 37 & -35 & -41\end{array}$

$\begin{array}{llllll}-5 & -1 & 18 & 51 & -27 & -35\end{array}$

$\begin{array}{llllll}-4 & -1 & 18 & 58 & -22 & -23\end{array}$

$\begin{array}{llllll}-3 & -1 & 18 & 58 & -11 & -25\end{array}$

$\begin{array}{rrrrrr}-2 & -1 & 18 & 79 & -13 & -23 \\ -1 & -1 & 3.8 & 87 & 18 & -19\end{array}$

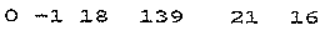

$\begin{array}{llllll}1 & -1 & 18 & 22 & -53 & -42\end{array}$

$\begin{array}{llllll}2 & -1 & 18 & 53 & 26 & -24 \\ 3 & -1 & 18 & 63 & 35 & -27\end{array}$

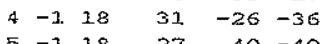

$\begin{array}{llllll}5 & -1 & 18 & 27 & 40 & -40\end{array}$

$\begin{array}{rrrrrr}6 & -1 & 18 & 25 & -19 & -34 \\ 7 & -1 & 18 & 41 & 7 & -35\end{array}$

$\begin{array}{llllll}8 & -1 & 18 & 61 & -46 & -24\end{array}$

$\begin{array}{rrrrrr}9 & -1 & 18 & 48 & -11 & -33 \\ -5 & 0 & 18 & 87 & -52 & -25\end{array}$

$\begin{array}{llllll}-4 & 0 & 18 & 100 & 80 & -21\end{array}$

$\begin{array}{llllll}-3 & 0 & 18 & 109 & -110 & 10\end{array}$

$\begin{array}{llllll}-2 & 0 & 18 & 112 & 93 & 10\end{array}$

$\begin{array}{rrrrrr}-1 & 0 & 18 & 162 & -132 & 12 \\ 0 & 0 & 18 & 218 & 208 & 6\end{array}$

$1018 \quad 200-196$

$\begin{array}{llllll}2 & 0 & 18 & 154 & 146 & 7\end{array}$

$\begin{array}{rrrrrr}4 & 0 & 18 & 195 & 180 & 7 \\ 5 & 0 & 18 & 174 & -154 & 8 \\ 6 & 0 & 18 & 198 & 178 & 6 \\ 7 & 0 & 18 & 129 & -101 & 13 \\ 8 & 0 & 18 & 84 & 58 & -24 \\ 9 & 0 & 18 & 59 & -53 & -19 \\ 10 & 0 & 18 & 34 & 45 & -26 \\ -5 & 1 & 18 & 40 & -14 & -34 \\ -4 & 1 & 18 & 69 & 8 & -25 \\ -3 & 1 & 18 & 37 & 5 & -36 \\ -2 & 1 & 18 & 43 & -30 & -29 \\ -1 & 1 & 18 & 30 & 55 & -32 \\ 0 & 1 & 18 & 41 & -14 & -33 \\ 1 & 1 & 18 & 83 & 25 & -22 \\ 2 & 1 & 18 & 67 & 19 & -22 \\ 3 & 1 & 18 & 50 & -26 & -28 \\ 5 & 1 & 18 & 46 & 17 & -26 \\ 6 & 1 & 18 & 61 & 34 & -21 \\ 7 & 1 & 18 & 63 & -37 & -21 \\ 8 & 1 & 18 & 76 & 15 & -16 \\ 9 & 1 & 18 & 79 & 20 & -18 \\ 10 & 1 & 18 & 72 & -13 & -25 \\ -5 & 2 & 18 & 90 & -86 & -19 \\ -4 & 2 & 18 & 46 & 65 & -34 \\ -3 & 2 & 18 & 91 & -76 & 15\end{array}$

$\begin{array}{rrrrrr}-3 & 2 & 18 & 91 & -76 & 15 \\ -2 & 2 & 18 & 81 & 82 & -17\end{array}$

$\begin{array}{rrrrrr}-1 & 2 & 18 & 149 & -134 & 31 \\ 0 & 2 & 18 & 222 & 213 & 8\end{array}$

$\begin{array}{llllll}1 & 2 & 18 & 171 & -183 & 10\end{array}$

$\begin{array}{rrrrrr}2 & 2 & 18 & 198 & 194 & 9 \\ 3 & 2 & 18 & 135 & -123 & 10\end{array}$

$\begin{array}{rrrrrr}3 & 2 & 18 & 135 & -123 & 10 \\ 4 & 2 & 18 & 124 & 96 & 11\end{array}$

$\begin{array}{llllll}5 & 2 & 18 & 164 & -167 & 10\end{array}$

$\begin{array}{llllll}7 & 2 & 18 & 133 & -137 & 13\end{array}$

$\begin{array}{llllrr}8 & 2 & 18 & 114 & 82 & 13\end{array}$

$\begin{array}{rrrrrr}9 & 2 & 18 & 95 & -79 & 16 \\ 10 & 2 & 18 & 39 & 61 & -34\end{array}$

$\begin{array}{rrrrrr}11 & 2 & 18 & 42 & -29 & -38\end{array}$

$\begin{array}{llllll}-4 & 3 & 18 & 71 & 6 & -19\end{array}$

$\begin{array}{rrrrrr}-3 & 3 & 18 & 72 & -17 & -26 \\ -2 & 3 & 18 & 119 & -10 & 17\end{array}$

$\begin{array}{rrrrrr}-1 & 3 & 18 & 48 & 5 & -23 \\ 0 & 3 & 18 & 44 & -27 & -29\end{array}$

$\begin{array}{llllll}1 & 3 & 18 & 92 & 15 & -19\end{array}$

$\begin{array}{rrrrrr}2 & 3 & 18 & 63 & -24 & -25 \\ 3 & 3 & 18 & 90 & 85 & 15\end{array}$

$\begin{array}{rrrrrr}3 & 3 & 18 & 90 & 85 & 15 \\ 4 & 3 & 18 & 26 & -25 & -38\end{array}$

$\begin{array}{rrrrrr}5 & 3 & 18 & 102 & 61 & 12\end{array}$

$\begin{array}{rrrrrr}7 & 3 & 18 & 12 & 28 & -50\end{array}$

$\begin{array}{rrrrrr}7 & 3 & 18 & 12 & 28 & -50 \\ 8 & 3 & 18 & 23 & -43 & -46\end{array}$

$\begin{array}{rrrrrr}9 & 3 & 18 & 46 & -4 & -27 \\ 10 & 3 & 18 & 40 & 18 & -37\end{array}$

$\begin{array}{llllll}11 & 3 & 18 & 66 & -26 & -28\end{array}$

$\begin{array}{llllll}-4 & 4 & 18 & 121 & 99 & 14\end{array}$

$\begin{array}{llllll}-3 & 4 & 2.8 & 58 & -79 & -24 \\ -2 & 4 & 18 & 24 & 75 & -45\end{array}$

$\begin{array}{rrrrrr}-1 & 4 & 18 & 83 & -95 & -18 \\ 0 & 4 & 18 & 163 & 156 & 10\end{array}$

$\begin{array}{llllll}1 & 4 & 18 & 234 & -230 & 8\end{array}$

$\begin{array}{llllll}2 & 4 & 18 & 168 & 154 & 9\end{array}$

$\begin{array}{rrrrrr}3 & 4 & 18 & 213 & -197 & 8 \\ 4 & 4 & 18 & 121 & 118 & 13\end{array}$

$\begin{array}{llllll}5 & 4 & 18 & 90 & -97 & 35\end{array}$

$\begin{array}{rrrrrr}6 & 4 & 18 & 142 & 121 & 11 \\ 7 & 4 & 18 & 80 & -83 & -18\end{array}$

$\begin{array}{rrrrrr}7 & 4 & 18 & 80 & -83 & -18 \\ 8 & 4 & 18 & 57 & 6 & -28\end{array}$

9418 81 $-35-18$

$\begin{array}{rrrrrr}10 & 4 & 18 & 63 & 22 & -28 \\ 11 & 4 & 18 & 58 & -27 & -31\end{array}$

$\begin{array}{rrrrrr}11 & 4 & 18 & 58 & -27 & -31 \\ -4 & 5 & 18 & 60 & -9 & -29\end{array}$

$\begin{array}{llllll}-3 & 5 & 18 & 59 & 21 & -23\end{array}$

$\begin{array}{llllll}-2 & 5 & 18 & 24 & 4 & -45 \\ -1 & 5 & 18 & 62 & 6 & -17\end{array}$

$\begin{array}{lllll}-5 & 18 & 69 & 24 & -17\end{array}$

$\begin{array}{llllll}1 & 5 & 18 & 44 & -2 & -29\end{array}$

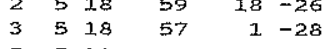

$\begin{array}{llllrl}5 & 5 & 18 & 48 & 5 & -28 \\ 6 & 5 & 18 & 73 & 26 & -23\end{array}$

$\begin{array}{rrrrrr}7 & 5 & 18 & 42 & -16 & -33\end{array}$

$\begin{array}{llllll}8 & 5 & 18 & 64 & 2 & -25 \\ 9 & 5 & 18 & 89 & 3 & 15\end{array}$

$\begin{array}{rrrrrr}10 & 5 & 18 & 61 & -1.8 & -27\end{array}$

$\begin{array}{rrrrrr}11 & 5 & 18 & 58 & 12 & -30 \\ -3 & 6 & 1.8 & 95 & -89 & 17\end{array}$

$\begin{array}{llllll}-2 & 6 & 18 & 110 & 94 & 15\end{array}$

$\begin{array}{rrrrrr}-1 & 6 & 18 & 88 & -82 & 16 \\ 0 & 6 & 18 & 132 & 129 & 11\end{array}$ $\begin{array}{llllll}1 & 6 & 18 & 151 & -158 & 11\end{array}$

$\begin{array}{llllll}2 & 6 & 18 & 137 & 136 & 11\end{array}$

$\begin{array}{llllll}4 & 6 & 18 & 151 & 171 & 11\end{array}$

$\begin{array}{llllll}5 & 6 & 18 & 128 & -126 & 12\end{array}$

$\begin{array}{rrrrrr}6 & 6 & 18 & 103 & 86 & 14 \\ 7 & 6 & 18 & 71 & -63 & -18\end{array}$

$\begin{array}{llllll}8 & 6 & 18 & 57 & 34 & -25\end{array}$

$\begin{array}{llllll}9 & 6 & 18 & 89 & -86 & 17\end{array}$

$\begin{array}{llllll}10 & 6 & 18 & 93 & 100 & 19\end{array}$

$\begin{array}{llllll}11 & 6 & 18 & 79 & -61 & -20 \\ -2 & 7 & 18 & 47 & -20 & -31\end{array}$

$\begin{array}{llllll}-1 & 7 & 18 & 75 & 39 & -20\end{array}$

$\begin{array}{llllll}0 & 7 & 18 & 82 & 0 & -23\end{array}$

$\begin{array}{rrrrrr}3 . & 7 & 18 & 51 & 7 & -27 \\ 2 & 7 & 18 & 45 & 12 & -29\end{array}$

$\begin{array}{llllll}3 & 7 & 18 & 68 & -35 & -23 \\ 4 & 7 & 18 & 38 & 14 & -32\end{array}$

$\begin{array}{lllllll}4 & 7 & 18 & 58 & 14 & -32 \\ 5 & 7 & 18 & 92 & -9 & -19\end{array}$

$\begin{array}{cccccc}5 & 7 & 18 & 92 & -9 & -19 \\ 6 & 7 & 18 & 89 & 43 & 14\end{array}$

$\begin{array}{llllll}7 & 18 & 40 & -27 & -33\end{array}$

$\begin{array}{llllll}8 & 7 & 18 & 37 & -12 & -35 \\ 9 & 7 & 18 & 24 & -14 & -43\end{array}$

$\begin{array}{llllll}10 & 7 & 18 & 51 & -4 & -26\end{array}$

$\begin{array}{llllll}11 & 7 & 18 & 35 & -11 & -35\end{array}$

$\begin{array}{rrrrrr}-1 & 8 & 18 & 200 & -88 & 16 \\ 0 & 8 & 18 & 106 & 107 & 15\end{array}$

$\begin{array}{rrrrrr}1 & 8 & 18 & 57 & -107 & -29\end{array}$

$\begin{array}{llllll}2 & 8 & 18 & 104 & 97 & 14\end{array}$

$\begin{array}{llllll}3 & 8 & 18 & 79 & -95 & -16\end{array}$

$\begin{array}{llllll}4 & 8 & 18 & 127 & 135 & 13\end{array}$

$\begin{array}{rrrrrr}5 & 8 & 1.8 & 134 & -140 & 12 \\ 6 & 8 & 18 & 77 & 99 & -18\end{array}$

$\begin{array}{llllll}7 & 8 & 18 & 57 & -72 & -24\end{array}$

$\begin{array}{llllll}8 & 8 & 18 & 45 & 52 & -35\end{array}$

$\begin{array}{rrrrrr}9 & 8 & 1.8 & 35 & -35 & -42 \\ 10 & 8 & 18 & 96 & 61 & 17\end{array}$

$\begin{array}{llllll}11 & 8 & 18 & 71 & -47 & -28\end{array}$

$\begin{array}{llllll}9 & 18 & 43 & -13 & -36\end{array}$

$\begin{array}{llllll}1 & 9 & 18 & 40 & -6 & -28\end{array}$

$\begin{array}{llllll}2 & 9 & 18 & 69 & -24 & -25 \\ 3 & 9 & 18 & 53 & -14 & -22\end{array}$

$\begin{array}{llllll}4 & 9 & 18 & 43 & 22 & -31\end{array}$

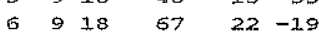

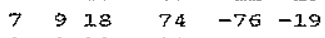

$\begin{array}{rrrrrr}8 & 9 & 18 & 38 & -9 & -35 \\ 9 & 9 & 18 & 39 & -47 & -37\end{array}$

$\begin{array}{llllll}10 & 9 & 18 & 74 & 28 & -20\end{array}$

$110 \quad 18$

21018

31018

$\begin{array}{rrr}74 & 28 & -20 \\ 98 & -87 & 16\end{array}$

$\begin{array}{lllll}10 & 18 & 107 & -110 & 16\end{array}$

$\begin{array}{rrrrrr}5 & 10 & 18 & 103 & -78 & 14\end{array}$

$\begin{array}{llllll}6 & 10 & 18 & 91 & 89 & 17\end{array}$

$\begin{array}{llllll}7 & 10 & 18 & 85 & -49 & 17\end{array}$

$\begin{array}{rrrrrr}8 & 10 & 28 & 30 & 55 & -40 \\ 9 & 10 & 18 & 110 & -82 & 15\end{array}$

$\begin{array}{rrrrrr}3 & 11 & 18 & 101 & -5 & -21 \\ 4 & 11 & 18 & 52 & -7 & -29\end{array}$

$\begin{array}{llllll}4 & 11 & 18 & 52 & -7 & -29 \\ 5 & 11 & 18 & 49 & -14 & -29\end{array}$

$\begin{array}{llllll}6 & 11 & 18 & 52 & 1 & -31\end{array}$

$\begin{array}{llllll}7 & 11 & 18 & 25 & -11 & -45\end{array}$

$\begin{array}{rrrrrr}8 & 31 & 18 & 69 & 8 & -29\end{array}$

$\begin{array}{llllll}-2 & -5 & 19 & 67 & 41 & -29\end{array}$

$\begin{array}{rrrrrr}0 & -5 & 19 & 86 & 75 & 17\end{array}$

$\begin{array}{llllll}1 & -5 & 19 & 72 & -92 & -30\end{array}$

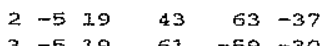

$\begin{array}{rrrrrr}4 & -5 & 19 & 105 & 49 & 14\end{array}$

$\begin{array}{llllll}-3 & -4 & 19 & 56 & -23 & -33\end{array}$

$\begin{array}{lllllll}-2 & -4 & 19 & 39 & -4 & -38\end{array}$

$\begin{array}{llllll}-1 & -4 & 19 & 76 & -21 & -27 \\ 0 & -4 & 19 & 59 & 44 & -21\end{array}$

$\begin{array}{llllll}0 & -4 & 19 & 59 & 44 & -21 \\ 1 & -4 & 19 & 48 & -1 & -32\end{array}$

$\begin{array}{llllll}1 & -4 & 19 & 48 & -21 & -32 \\ 3 & -4 & 19 & 97 & -22 & -20\end{array}$

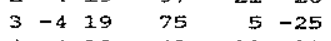

$\begin{array}{llllll}4 & -4 & 19 & 43 & -33 & -31 \\ 6 & -4 & 19 & 66 & -19 & -29\end{array}$

$\begin{array}{llllll}-3 & -3 & 19 & 92 & -79 & 16\end{array}$

$\begin{array}{llllll}-2 & -3 & 19 & 131 & 77 & 12\end{array}$

$\begin{array}{llllll}-1 & -3 & 19 & 83 & -65 & -17\end{array}$

$\begin{array}{rrrrrr}0 & -3 & 19 & 64 & 52 & -27 \\ 1 & -3 & 19 & 1.16 & -93 & 12\end{array}$

$\begin{array}{llllll}2 & -3 & 19 & 157 & 139 & 13\end{array}$

$\begin{array}{llllll}3 & -3 & 19 & 161 & -131 & 11\end{array}$

$\begin{array}{llllll}4 & -3 & 19 & 109 & 102 & 15\end{array}$

$\begin{array}{rrrrrr}5 & -3 & 19 & 202 & -63 & 14 \\ 6 & -3 & 19 & 40 & 50 & -37\end{array}$

$\begin{array}{llllll}7 & -3 & 19 & 58 & -46 & -27\end{array}$

$\begin{array}{llllll}7 & -2 & 19 & 51 & 28 & -33\end{array}$

$\begin{array}{llllll}-2 & -2 & 19 & 34 & 5 & -40\end{array}$

$\begin{array}{llllll}-1 & -2 & 19 & 111 & 28 & 19\end{array}$

$\begin{array}{rrrrrr}0 & -2 & 19 & 121 & -4 & 17 \\ 1 & -2 & 19 & 64 & -26 & -25\end{array}$

$\begin{array}{llllll}2 & -2 & 19 & 51 & -44 & -28\end{array}$

$\begin{array}{llllll}3 & -2 & 19 & 12 & 31 & -46\end{array}$

$\begin{array}{llllll}4 & -2 & 19 & 49 & -9 & -31 \\ 5 & -2 & 19 & 73 & 25 & -24\end{array}$

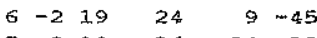

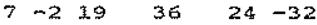

$\begin{array}{llllll}8 & -2 & 19 & 58 & -38 & -33\end{array}$

$\begin{array}{rrrrrr}-4 & -1 & 19 & 36 & 64 & -38 \\ -3 & -1 & 19 & 101 & -86 & 15\end{array}$

$\begin{array}{llllll}-2 & -1 & 19 & 138 & 93 & 11\end{array}$

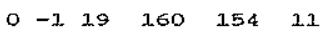

$\begin{array}{llllll}1 & -1 & 19 & 129 & -122 & 12\end{array}$

$\begin{array}{llllll}2 & -1 & 19 & 98 & 102 & 15\end{array}$

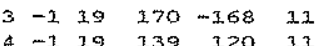

$\begin{array}{llllll}5 & -1 & 19 & 137 & -105 & 11\end{array}$

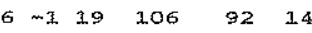

$\begin{array}{llllll}7 & -1 & 19 & 78 & -65 & -19\end{array}$

$\begin{array}{rrrrrr}8 & -1 & 19 & 65 & 43 & -23 \\ 9 & -1 & 19 & 61 & -26 & -30\end{array}$

$\begin{array}{llllll}-4 & 0 & 19 & 80 & 30 & -30\end{array}$

$\begin{array}{llllll}-3 & 0 & 19 & 48 & -19 & -20\end{array}$

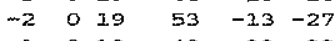

$\begin{array}{rrrrrr}-1 & 0 & 19 & 48 & 33 & -22 \\ 0 & 0 & 39 & 123 & -30 & -34\end{array}$

$\begin{array}{llllll}1 & 0 & 19 & 122 & 25 & -12\end{array}$ 


\begin{tabular}{|c|c|c|c|c|c|c|c|c|c|c|c|c|}
\hline th & $k_{2}$ & 1 & 10Fo & $10 \mathrm{FC}$ & $10 \mathrm{~s}$ & h & $\mathrm{k}$ & 1 & 1OFO & $10 F C$ & $10 \mathrm{~s}$ & $\mathrm{n}$ \\
\hline 3 & 4 & 19 & 108 & 22 & 17 & 0 & -3 & 20 & 89 & 34 & -23 & 6 \\
\hline 4 & 4 & 19 & 38 & -34 & -32 & 3 & -3 & 20 & 50 & -5 & -28 & 7 \\
\hline 5 & 4 & 3.9 & 45 & 41 & -24 & 4 & -3 & 20 & 55 & -24 & -29 & 8 \\
\hline 7 & 4 & 19 & $4 G$ & -4 & -31 & 5 & -3 & 20 & 58 & 26 & -24 & 10 \\
\hline 8 & 4 & 29 & 24 & -8 & -42 & 5 & -3 & 20 & 32 & -2 & -41 & -2 \\
\hline 9 & 4 & 19 & 54 & 25 & -24 & -2 & -2 & 20 & 130 & 95 & 13 & -1 \\
\hline 0 & 4 & 18 & 76 & -20 & -28 & -1 & -2 & 20 & 147 & -104 & 11 & 0 \\
\hline 1 & 4 & 19 & 62 & -12 & -28 & o & -2 & 20 & 130 & 131 & 13 & 1 \\
\hline-3 & 5 & 19 & 134 & -92 & 13 & . & -2 & 20 & 124 & -103 & 13 & 2 \\
\hline-2 & 5 & 19 & 49 & 78 & -34 & 2 & -2 & 20 & 102 & 95 & 15 & 3 \\
\hline-1 & 5 & 19 & 78 & -82 & -20 & 3 & -2 & 20 & 78 & -88 & -20 & 4 \\
\hline 0 & 5 & 19 & 151 & 122 & 11 & 4 & -2 & 20 & 38 & 64 & -41 & 5 \\
\hline 1 & E & 19 & 141 & -112 & $I I$ & 5 & -2 & 20 & 99 & -87 & 16 & 6 \\
\hline 2 & 5 & 19 & 93 & 101 & 15 & 6 & -2 & 20 & 102 & 94 & 16 & 7 \\
\hline 3 & 5 & 19 & 185 & -170 & 9 & 7 & -2 & 20 & 32 & -55 & -42 & 8 \\
\hline 4 & 5 & 19 & 106 & 129 & 15 & -3 & -1 & 20 & 93 & -14 & -21 & 9 \\
\hline 5 & 5 & 19 & 117 & -106 & 12 & -2 & -1 & 20 & 42 & 26 & -34 & 10 \\
\hline 6 & 5 & 19 & 336 & 82 & 21 & -1 & -1 & 20 & 54 & -19 & -35 & -1 \\
\hline 7 & 5 & 29 & 51 & -37 & -30 & 0 & -1 & 20 & 122 & 21 & 18 & 0 \\
\hline 8 & 5 & 19 & 69 & 15 & -25 & 1 & -1 & 20 & 57 & -9 & -29 & 1 \\
\hline 9 & 5 & 19 & 80 & -43 & -16 & 3 & -1 & 20 & 57 & 10 & -31 & 2 \\
\hline 0 & 5 & 19 & 31 & 42 & -46 & 4 & -1 & 20 & 10 & -5 & -38 & 3 \\
\hline 1 & 5 & 19 & 61 & -16 & -28 & 5 & -1 & 20 & 56 & 1.1. & -28 & 4 \\
\hline-2 & 6 & 19 & 56 & -14 & -31 & 6 & -1 & 20 & 49 & -4 & -30 & 5 \\
\hline-1 & 6 & 19 & 83 & -9 & -21 & 7 & -1 & 20 & 47 & 6 & -33 & 6 \\
\hline 1 & 6 & 19 & 94 & 7 & 19 & 8 & -1 & 20 & 32 & -5 & -42 & 7 \\
\hline 2 & 6 & 19 & 53 & -16 & -27 & -3 & 0 & 20 & 86 & -46 & 14 & 8 \\
\hline 3 & 6 & 19 & 35 & 31 & -35 & -2 & 0 & 20 & 90 & 81 & 14 & 9 \\
\hline 4 & 6 & 19 & 83 & 26 & -23 & -1 & 0 & 20 & 131 & -93 & 9 & 10 \\
\hline 5 & 6 & 19 & 12 & -53 & -48 & 0 & 0 & 20 & 154 & 125 & 12 & 0 \\
\hline 6 & $\sigma$ & 19 & 12 & 33 & -48 & 1 & 0 & 20 & 152 & -136 & 8 & 1 \\
\hline 7 & 6 & 19 & 63 & 19 & -27 & 2 & o & 20 & 98 & 98 & .11 & 2 \\
\hline 8 & 6 & 19 & 48 & -25 & -29 & 3 & 0 & 20 & 215 & -94 & 13 & 3 \\
\hline 9 & 6 & 19 & 46 & 24 & -32 & 4 & 0 & 20 & 83 & 95 & 22 & 4 \\
\hline 0 & 6 & 19 & 31 & -2 & -43 & 5 & 0 & 20 & 108 & -88 & 9 & 5 \\
\hline 2 & 6 & 19 & 59 & 6 & -24 & $G$ & 0 & 20 & 206 & 204 & 13 & 6 \\
\hline-1 & 7 & 19 & 118 & -106 & 15 & 7 & 0 & 20 & 103 & -90 & 22 & 7 \\
\hline 0 & 7 & 19 & 102 & 103 & 1.5 & 8 & 0 & 20 & 96 & 27 & -20 & 8 \\
\hline 1 & 7 & 19 & 136 & -135 & 12 & -3 & 1 & 20 & 25 & 6 & -47 & 9 \\
\hline 2 & 7 & 19 & 44 & 62 & -26 & -2 & 1 & 20 & 78 & 3 & -17 & 20 \\
\hline 3 & 7 & 19 & 313 & -98 & 11 & -1 & 1 & 20 & 69 & 7 & -23 & 1 \\
\hline 4 & 7 & 19 & 130 & 101 & 12 & 0 & 1 & 20 & 110 & 15 & 18 & 2 \\
\hline 5 & 7 & 29 & 201 & -95 & 15 & 1 & 1 & 20 & 79 & -15 & -22 & 3 \\
\hline 6 & 7 & 19 & 127 & 110 & 12 & 2 & 1 & 20 & 66 & -30 & -24 & 4 \\
\hline 7 & 7 & 19 & 120 & -130 & 13 & 3 & 1 & 20 & 95 & 8 & 28 & 5 \\
\hline 8 & 7 & 19 & 24 & 24 & -42 & 4 & 1 & 20 & 28 & -18 & -39 & 6 \\
\hline 9 & 7 & 19 & 30 & -8 & -41 & 5 & 1 & 20 & 31 & 3.3 & -30 & 7 \\
\hline .0 & 7 & 19 & 25 & 17 & -49 & 6 & 3. & 20 & 55 & 30 & -27 & 8 \\
\hline 0 & 8 & 19 & 39 & 13 & -36 & 7 & 1 & 20 & 84 & -3 & -22 & 9 \\
\hline 1 & 8 & 19 & 24 & -24 & -44 & 8 & 1 & 20 & 31 & -13 & -43 & 2 \\
\hline 2 & 8 & 19 & 47 & -20 & -31 & 9 & 1 & 20 & 55 & -8 & -31 & 3 \\
\hline 3 & 8 & 19 & 12 & -8 & -47 & -3 & 2 & 20 & 12 & -50 & -50 & 4 \\
\hline 4 & 8 & 19 & 97 & -21 & -20 & -2 & 2 & 20 & 126 & 63 & 12 & 5 \\
\hline 5 & 8 & 19 & 75 & 8 & -16 & -1 & 2 & 20 & 112 & -80 & 13 & 6 \\
\hline 6 & 8 & 19 & 33 & 46 & -37 & 0 & 2 & 20 & 138 & 140 & 12 & 7 \\
\hline 7 & 8 & 19 & 73 & -18 & -27 & 1 & 2 & 20 & 125 & -134 & 13 & 8 \\
\hline 8 & 8 & 19 & 38 & 1 & -33 & 2 & 2 & 20 & 101 & 84 & 13 & 4 \\
\hline 9 & 8 & 29 & 88 & -24 & -24 & 3 & 2 & 20 & 109 & -87 & 12 & 5 \\
\hline 0 & 8 & 19 & 36 & -23 & -40 & 4 & 2 & 20 & 89 & 74 & 1.4 & 6 \\
\hline 1 & 9 & 19 & 94 & -92 & 27 & 5 & 2 & 20 & 122 & -95 & 32 & 7 \\
\hline 2 & 9 & 19 & 34 & 55 & -36 & 6 & 2 & 20 & 119 & 106 & 12 & $\circ$ \\
\hline 3 & 9 & 19 & 24 & $-7 I$ & -44 & 7 & 2 & 20 & 56 & -88 & -32 & $x$ \\
\hline$\Delta$ & 9 & 19 & 75 & 67 & -18 & 8 & 2 & 20 & 106 & 85 & 25 & 2 \\
\hline 5 & 9 & 19 & 325 & -99 & 12 & 9 & 2 & 20 & 59 & -63 & -33 & 3 \\
\hline 6 & 9 & 19 & 80 & 77 & -18 & -3 & 3 & 20 & 25 & -15 & -43 & 4 \\
\hline 7 & 9 & 19 & 91. & -63 & 16 & -2 & 3 & 20 & 43 & 0 & -35 & -1 \\
\hline 8 & 9 & 19 & 85 & 58 & 16 & -1 & 3 & 20 & 45 & 16 & -31 & 0 \\
\hline 9 & 9 & 19 & 50 & -42 & -34 & 0 & 3 & 20 & 66 & 17 & -27 & 1 \\
\hline 2 & 10 & 19 & 12 & -13 & -48 & 1 & 3 & 20 & 71 & -14 & -23 & 2 \\
\hline 3 & 10 & 19 & 66 & -12 & -20 & 2 & 3 & 20 & 77 & 20 & -24 & 3 \\
\hline$\frac{4}{2}$ & 20 & 19 & 35 & 17 & -36 & 3 & 3 & 20 & 11 & -35 & -45 & 4 \\
\hline 5 & 10 & 19 & 59 & -9 & -29 & 4 & 3 & 20 & 81 & -12 & -19 & 5 \\
\hline 6 & 10 & 19 & 13 & 17 & -51 & 5 & 3 & 20 & 28 & 16 & -40 & -2 \\
\hline 7 & 10 & 19 & 39 & -23 & -35 & 6 & 3 & 20 & 80 & 19 & -21 & 0 \\
\hline 8 & 10 & 19 & 87 & -10 & -24 & 7 & 3 & 20 & 33 & -14 & -40 & 1 \\
\hline 9 & 10 & 19 & 60 & -15 & -28 & 8 & 3 & 20 & 13 & -16 & -51 & 2 \\
\hline 5 & 21 & 19 & 61 & -59 & -22 & 9 & 3 & 20 & 13 & 13 & -50 & 3 \\
\hline 6 & II & 19 & 1.11 & 89 & 14 & 10 & 3 & 20 & 26 & 18 & -44 & 4 \\
\hline 1 & -4 & 20 & 135 & -82 & 33 & -2 & 4 & 20 & 82 & 76 & -39 & 5 \\
\hline 0 & -4 & 20 & 27 & 84 & -38 & -1 & 4 & 20 & 11主 & -93 & 34 & 6 \\
\hline 1 & -4 & 20 & 106 & -93 & 36 & 0 & 4 & 20 & 110 & 109 & 2.4 & -2 \\
\hline 2 & -4 & 20 & 61 & 68 & -29 & 1 & 4 & 20 & 99 & -93 & 14 & -1 \\
\hline 3 & -4 & 20 & 80 & -63 & -17 & 2 & 4 & 20 & 138 & 113 & 13 & 0 \\
\hline & -4 & 20 & 82 & 66 & -20 & 3 & 4 & 20 & 101 & -81 & 13 & 1 \\
\hline & -3 & 20 & 75 & 7 & -28 & 4 & 4 & 20 & 91 & 81 & 14 & 2 \\
\hline
\end{tabular}

